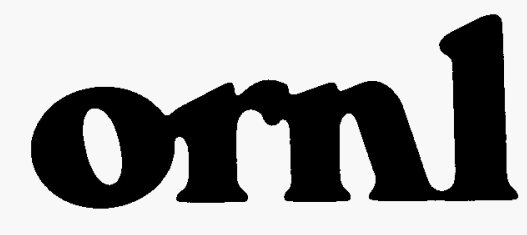

OAK RIDGE

NATIONAL

LABORATORY

MARTHW MARIETRA
RECEIVED

MAR 131996

OS.TI

\title{
Advanced Neutron Source Reactor Thermal Analysis of Fuel Plate Defects
}

\author{
G. E. Giles
}

August 1995

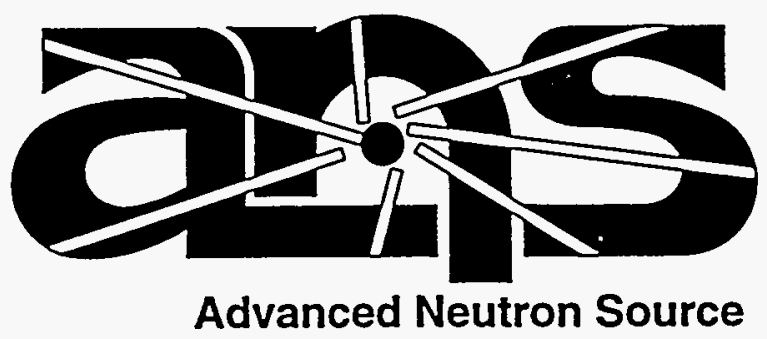

MANAGED BY

MARTIN MARIETTA ENERGY SYSTEMS, INC.

FOR THE UNITED STATES

DEPARTMENT OF ENERGY 
This report has been reproduced directly from the best available copy.

Available to DOE and DOE contractors from the Office of Scientific and Technical Information, P.O. Box 62, Oak Ridge, TN 37831; prices available from (615) 576-8401, FTS 626-8401.

Available to the public from the National Technical Information Service, U.S. Department of Commerce, 5285 Port Royal Rd., Springfield, VA 22161.

This report was prepared as an account of work sponsored by an agency of the United States Government. Neither the United States Government nor any agency thereof, nor any of their employees, makes any warranty, express or implied, or assumes any legal liability or responsibility for the accuracy, completeness, or usefulness of any information, apparatus, product, or process disclosed, or represents that its use would not infringe privately owned rights. Reference herein to any specific commercial product, process, or service by trade name, trademark, manufacturer, or otherwise, does not necessarily constitute or imply its endorsement, recommendation, or favoring by the United States Government or any agency thereof. The views and opinions of authors expressed herein do not necessarily state or reflect those of the United States Government or any agency thereof. 
Computational Physics and Engineering Division

\title{
ADVANCED NEUTRON SOURCE REACTOR THERMAL ANALYSIS OF FUEL PLATE DEFECTS
}

\author{
G. E. Giles
}

Date Completed: August 1995

Date Published: August 1995

Prepared by the

OAK RIDGE NATIONAL LABORATORY managed by

LOCKHEED MARTIN ENERGY SYSTEMS

for the U.S. Department of Energy

under contract DE-AC05-84OR21400

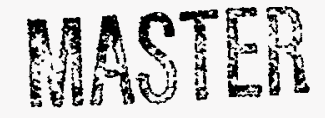

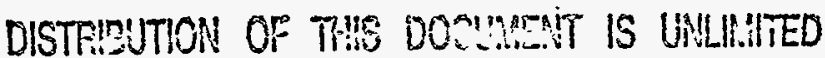




\section{CONTENTS}

Page

LIST OF FIGURES $\ldots \ldots \ldots \ldots \ldots \ldots \ldots \ldots \ldots \ldots \ldots \ldots \ldots \ldots \ldots \ldots \ldots$ vi

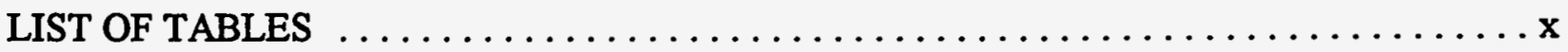

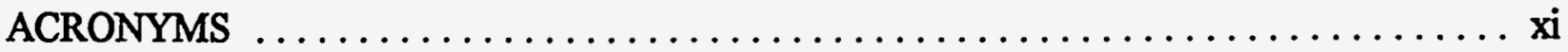

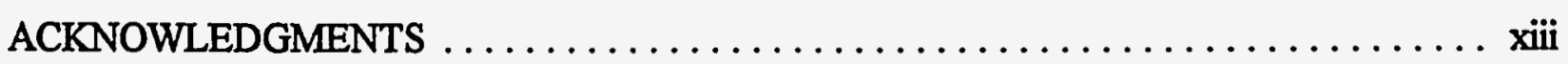

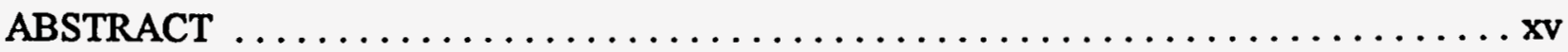

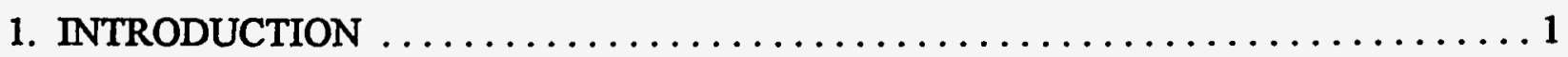

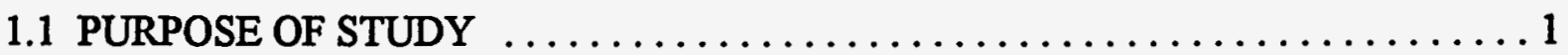

1.2 ANSR FUEL PLATE DESIGN $\ldots \ldots \ldots \ldots \ldots \ldots \ldots \ldots \ldots \ldots \ldots \ldots \ldots \ldots \ldots$

1.3 FUEL TEMPERATURE CRITERION $\ldots \ldots \ldots \ldots \ldots \ldots \ldots \ldots \ldots \ldots \ldots$

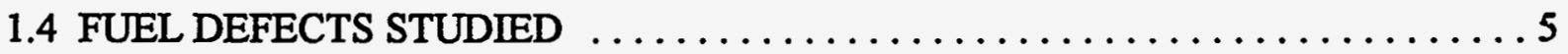

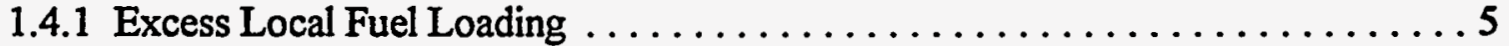

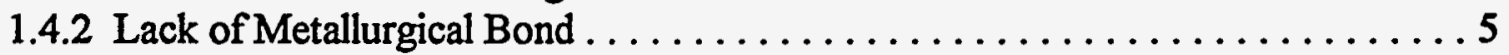

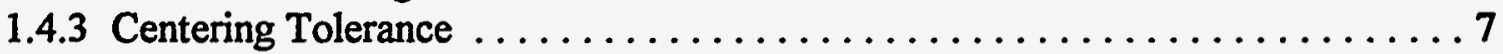

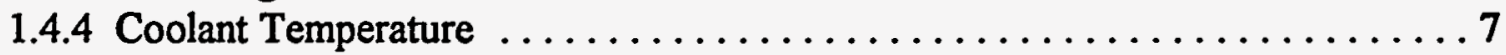

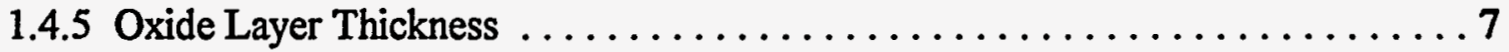

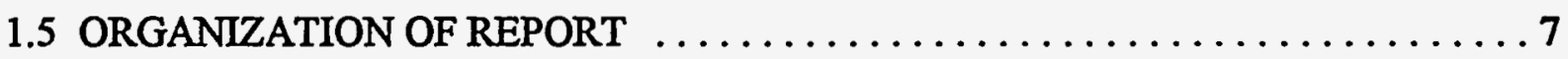

2. PREVIOUS ANALYSES OF FUEL DEFECTS $\ldots \ldots \ldots \ldots \ldots \ldots \ldots$

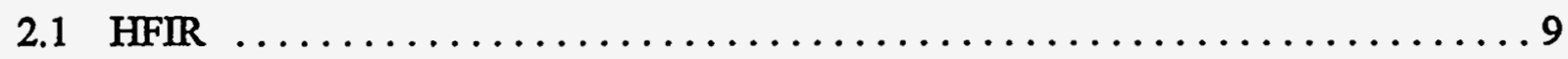

2.1.1 Hilvety and Chapman Study (ORNL-TM-1903, 1967) ............9

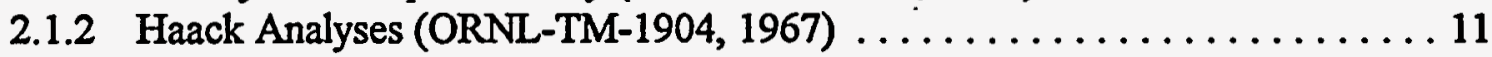

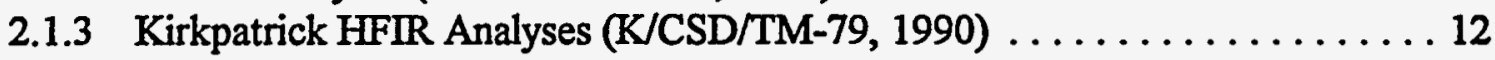

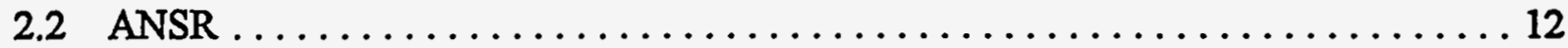

2.2.1 Kirkpatrick ANSR Analysis (Internal Correspondence, 1989) . . . . . . 12

3. PREVIOUS ANSR (GILES) ANALYSES $\ldots \ldots \ldots \ldots \ldots \ldots \ldots \ldots \ldots \ldots \ldots \ldots \ldots \ldots$

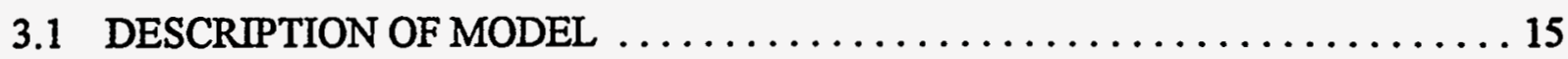

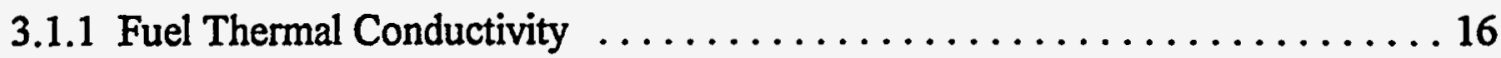


3.1.2 Fixed Thermal Conductivities ........................ 19

3.1.3 Heat Generation ................................... 19

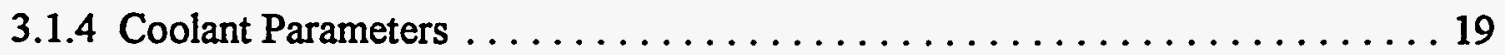

3.1.5 Excess Local Power . . . . . . . . . . . . . . . . . . . . . . . 21

3.2 PHENOMENA NOT INCLUDED IN THESE INVESTIGATIONS $\ldots \ldots \ldots \ldots 23$

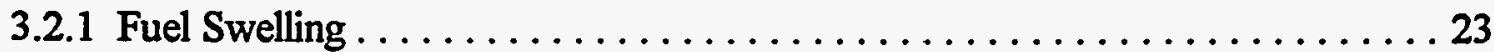

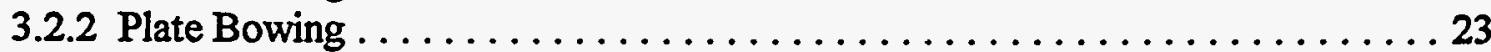

3.3 MEAT THICKNESS VARIATION ANALYSES $\ldots \ldots \ldots \ldots \ldots \ldots \ldots \ldots$

3.4 ANALYSES USING DESIGN LIMITS AS CONSERVATIVE ESTIMATE . . . . . 27

3.5 ANALYSES USING L7 CORE DESIGN WORST-CASE VALUES $\ldots \ldots \ldots \ldots 30$

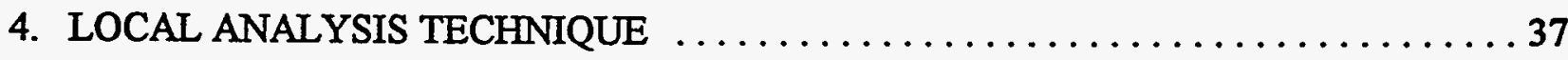

4.1 DISCUSSION OF PREVIOUS ASSUMPTIONS $\ldots \ldots \ldots \ldots \ldots \ldots \ldots \ldots \ldots$

4.2 IMPROVED ANALYSIS TECHNIQUE $\ldots \ldots \ldots \ldots \ldots \ldots \ldots \ldots \ldots \ldots \ldots \ldots$

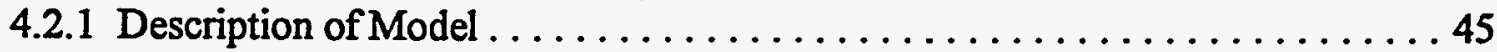

4.2.2 Uncertainty Levels Used in the Analysis $\ldots \ldots \ldots \ldots \ldots \ldots \ldots \ldots . \ldots . \ldots . \ldots 45$

4.3 INVESTIGATIONS OF NOMINAL VALUES USED IN THE LAT $\ldots \ldots \ldots \ldots 46$

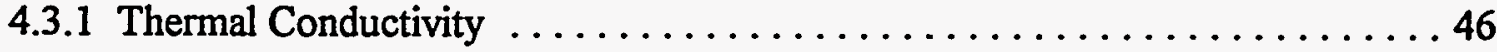

4.3.2 Oxide Layer Thickness Growth Model $\ldots \ldots \ldots \ldots \ldots \ldots \ldots \ldots \ldots$

4.3.3 Time Discretization . . . . . . . . . . . . . . . . . . . . . . . . 47

4.3.4 Spatial Discretization .......................... 47

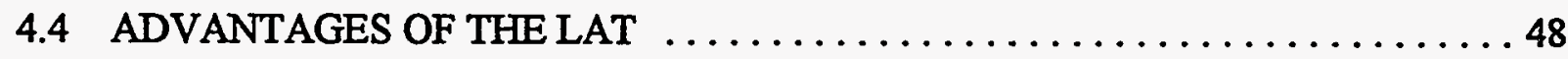

5. L7 CORE DESIGN LAT RESULTS $\ldots \ldots \ldots \ldots \ldots \ldots \ldots \ldots \ldots \ldots \ldots \ldots \ldots \ldots \ldots \ldots \ldots$

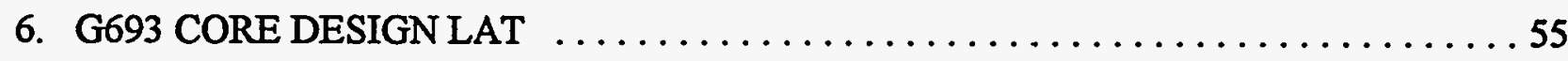

7. ANSR FUEL TEMPERATURE WORKSHOP $\ldots \ldots \ldots \ldots \ldots \ldots \ldots \ldots \ldots \ldots \ldots \ldots \ldots \ldots \ldots$

7.1 G693 ANALYSIS CONVERSION OF FUEL TO U(A.1,SI $)_{3} \ldots \ldots \ldots \ldots \ldots 6$

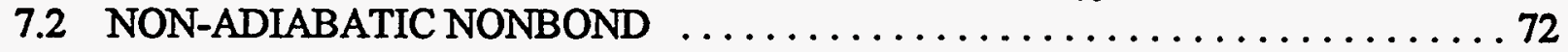


CONTENTS (continued)

Page

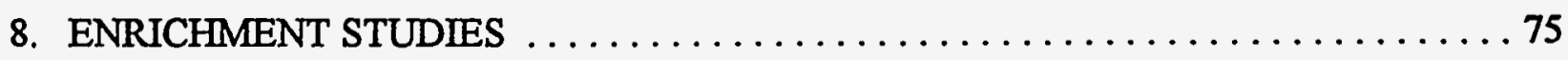

8.1 TEMPERATURE DEPENDENCY OF FUEL THERMAL CONDUCTIVITIES . . . 78

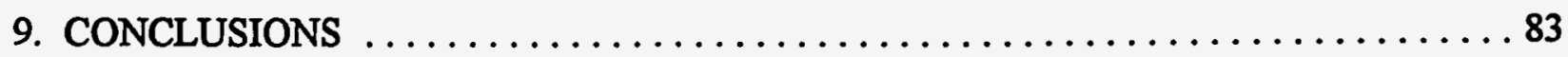

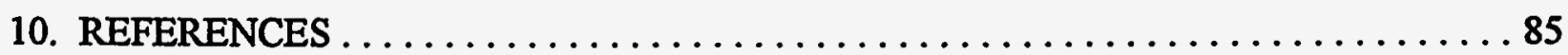

Appendix A. AUTOMATIC EXECUTION OF LAT ANALYSES $\ldots \ldots \ldots \ldots \ldots \ldots$ A.1

A.1 RD-ALL INPUT FILES DESCRIPTIONS $\ldots \ldots \ldots \ldots \ldots \ldots \ldots \ldots \ldots$ A.2

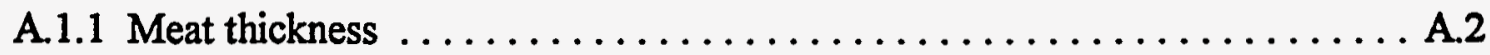

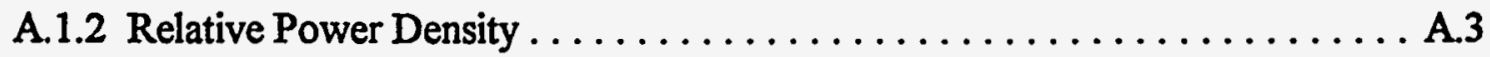

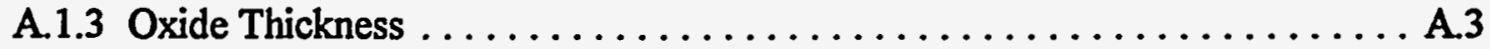

A.1.4 Coolant Bulk Temperature $\ldots \ldots \ldots \ldots \ldots \ldots \ldots \ldots \ldots \ldots \ldots \ldots \ldots \ldots \ldots \ldots \ldots$

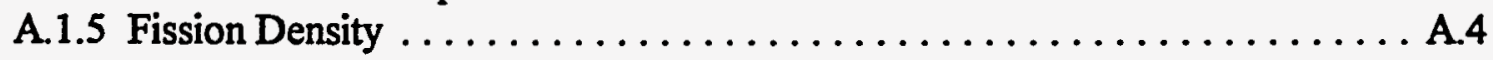

A.2 RD-ALL OUTPUT FILE DESCRIPTION $\ldots \ldots \ldots \ldots \ldots \ldots \ldots \ldots \ldots$ A.5

A.3 RD-ALL CODE LISTING $\ldots \ldots \ldots \ldots \ldots \ldots \ldots \ldots \ldots \ldots \ldots \ldots \ldots \ldots \ldots \ldots \ldots \ldots$

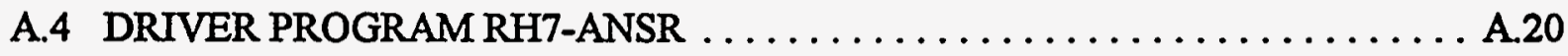

A.5 CODE LISTING FOR RH7-ANSR $\ldots \ldots \ldots \ldots \ldots \ldots \ldots \ldots \ldots \ldots \ldots \ldots \ldots \ldots \ldots \ldots$

Appendix B. CRITICAL HEAT FLUX ANALYSIS $\ldots \ldots \ldots \ldots \ldots \ldots \ldots \ldots$ B. 1

Appendix C. FUNCTIONAL REPLACEMENT FOR HEATING ANALYSIS . . . . . . C.1

Appendix D. ADVANCED NEUTRON SOURCE REACTOR CORE DESIGN DATA ... D.1

D.1 L7 CORE DESIGN FUEL PLATES $\ldots \ldots \ldots \ldots \ldots \ldots \ldots \ldots \ldots \ldots \ldots \ldots \ldots \ldots$

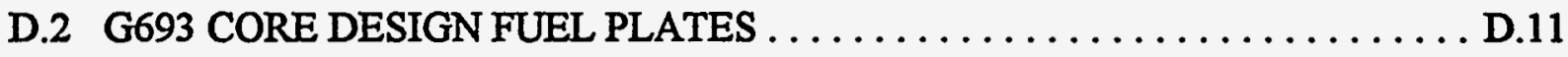

Appendix E. LOCAL TIME-DEPENDENT FISSION DENSITY $\ldots \ldots \ldots \ldots \ldots \ldots$ E. 1

E.1 METHOD OF CALCULATION $\ldots \ldots \ldots \ldots \ldots \ldots \ldots \ldots \ldots \ldots \ldots \ldots \ldots \ldots \ldots \ldots \ldots \ldots$

E.2 GENERATION OF FISSION DENSITY DATA $\ldots \ldots \ldots \ldots \ldots \ldots \ldots \ldots$ E.3 


\section{LIST OF FIGURES}

Figure

Page

1.1. ANSR fuel elements: vertical midplane section $\ldots \ldots \ldots \ldots \ldots \ldots \ldots \ldots \ldots \ldots$

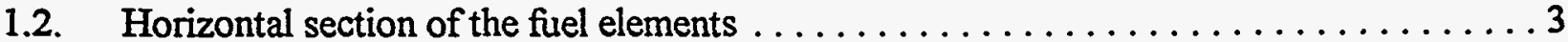

1.3. Section of the ANSR fuel plate showing placement of fuel meat within fuel volume for (a) offset, and (b) centered fuel meat design $\ldots \ldots \ldots \ldots \ldots \ldots \ldots \ldots$

1.4. Segregation volume and nonbond compared with fuel particle size range $\ldots \ldots \ldots \ldots 6$

2.1. Hilvety and Chapman thermal model $\ldots \ldots \ldots \ldots \ldots \ldots \ldots \ldots \ldots \ldots \ldots \ldots$

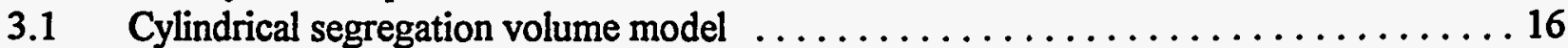

3.2. Comparison of thermal conductivity of normal fuel and segregated fuel as a function of fission density. Both sets of results were produced by the DART code $\ldots \ldots \ldots 18$

3.3. Comparison of thermal conductivity of segregated fuel and unirradiated $\mathrm{U}_{3} \mathrm{Si}_{2} \ldots \ldots \ldots 18$

3.4. Segregation volume models for mzximum meat layer thickness, (a) cylinder,

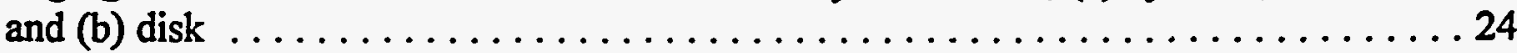

3.5. Comparison of peaking factor between models using a cylinder of disk segregation

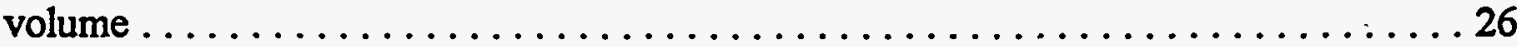

3.6. Comparison of maximum temperatures between models using a cylinder or disk

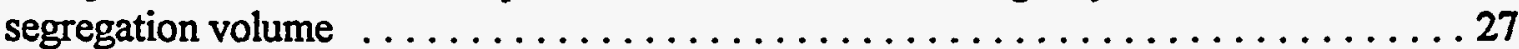

3.7. Peaking factor as a function of meat thickness for combinations of fuel defects . . . . 228

3.8. Maximum fuel temperature as a function of meat thickness for combinations of fuel defects .......................................29

3.9. Peaking factor as a function of meat thickness for design limit values with maximum

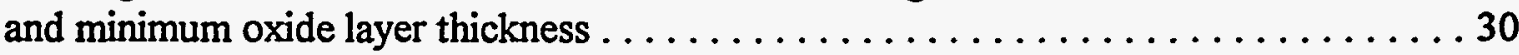

3.10. Maximum temperature as a function of meat thickness for design limits values with maximum and minimum oxide layer thickness . . . . . . . . . . . . . . 31

3.11. Comparison of the peaking factors for models with fuel meat layers that were centered

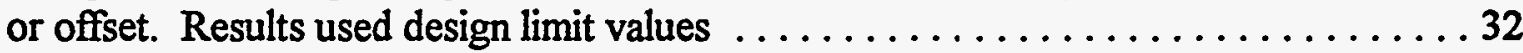

3.12. Comparison of the maximum temperatures for models with fuel meat layers that were centered or offset. Results used design limit values $\ldots \ldots \ldots \ldots \ldots \ldots \ldots \ldots 33$

3.13. Maximum peaking factors for design values is more conservative than the actual

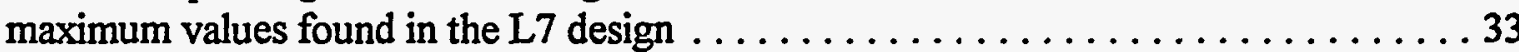

3.14. Maximum temepratures for design limits at maximum and minimum oxide thickness compared with worst-case values from the L7 core design. Maximum temperature for design values with no oxide is about the same as those for the actual maximum values

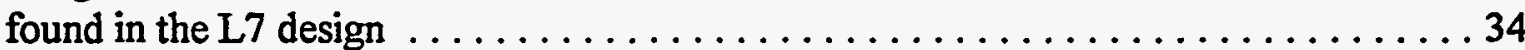

3.15. Peaking factors comparison for offset models. $L 7$ worst-case parameters $\ldots \ldots \ldots \ldots 35$

3.16. Meat layer at centered, $50 \%$ offset, and $100 \%$ offset positions ............. 36

4.1. Maximum and minimum relative power density as a function of time for the $\mathrm{L} 7$ core

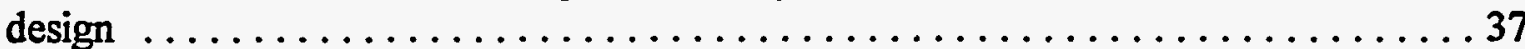

4.2. Contours of relative power density on upper and lower fuel plates for the L7 core

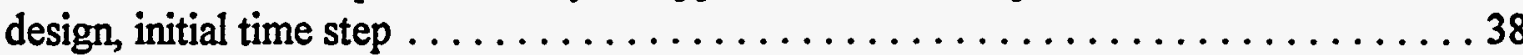




\section{LIST OF FIGURES (continued)}

4.3. Thermal conductivity of normal and segregated fuel as a function of time for the L7

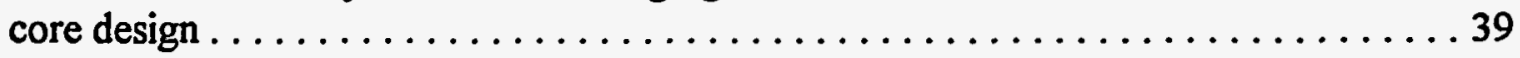

4.4. Maximum and minimum oxide layer thickness as a function of time for the $\mathrm{L} 7$ core

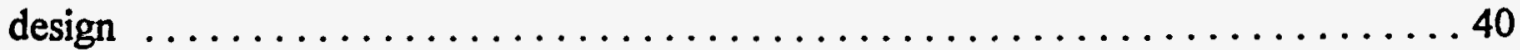

4.5. Maximum and minimum coolant temperature as a function of time for the L7 core

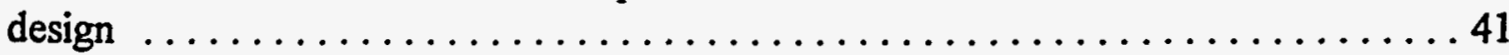

4.6. Grid from VENTURE analysis of ANSR L7 fuel plates $\ldots \ldots \ldots \ldots \ldots \ldots \ldots \ldots$

4.7. Meat thickness design grid for ANSR $L 7$ fuel plates $\ldots \ldots \ldots \ldots \ldots \ldots \ldots \ldots 44$

5.1. Peaking factor results from the LAT analysis of the L7 core design compared with design limit results as a function of meat thickness $\ldots \ldots \ldots \ldots \ldots \ldots \ldots . \ldots 49$

5.2. Maximum temperature results from the LAT analysis of the L7 core design compared with design limit results as a function of meat thickness $\ldots \ldots \ldots \ldots \ldots \ldots \ldots 50$

5.3. Maximum peaking factors from L7 core design LAT analysis compared with the

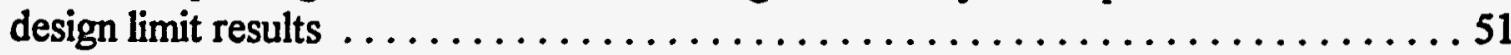

5.4. Comparison of $\mathrm{L} 7$ maximum temperatures with design limit analysis $\ldots \ldots \ldots \ldots 52$

5.5. Contours of peaking factor on $\mathrm{L} 7$ fuel plates. First time step of LAT analysis. The

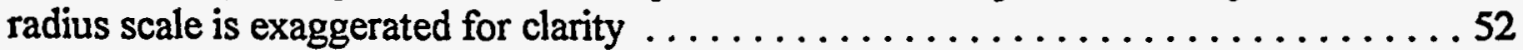

5.6. Contours of maximum temperature in the segregation volume on $L 7$ fuel plates, first time step of LAT analysis . . . . . . . . . . . . . . . . . . . 53

5.7. Carpet plot of maximum temperature in the segregation volume on $\mathrm{L} 7$ fuel plates, first

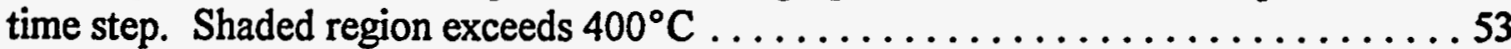

5.8. Contour plot of maximum temperature in the segregation volume on $L 7$ fuel plates drawn to scale. First time step of ALT analysis. Shaded region exceeds $400^{\circ} \mathrm{C} \ldots .54$

6.1. Peaking factor results from the LAT analysis of the G693 core design compared with

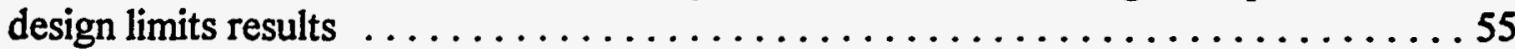

6.2. Maximum temperature results in the segregation volume from the LAT analysis of the G693 core design compared with design limits results as a function of meat thickness . 56

6.3. Maximum peaking factors from $\mathrm{G} 693$ core design LAT analysis compared with the

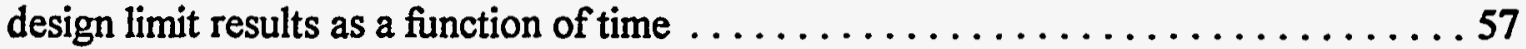

6.4. Maximum temepratures from G693 core design LAT analysis compared with the

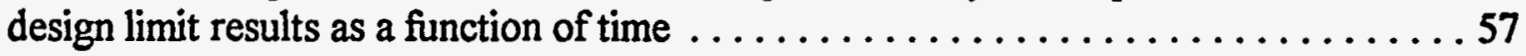

6.5. Contours of peaking factor on G693 fuel plates, first time step of LAT analysis . . . . 58

6.6. Contours of maximum temperature in segregation volume on G693 fuel plates, first time step of Lat anlaysis . . . . . . . . . . . . . . . . . . . . 59

6.7. Carpet plot of maximum temeprature in segregation volume on $\mathrm{G} 693$ fuel plates, first time step of LAT analysis. Shaded region exceeds $400^{\circ} \mathrm{C} \ldots \ldots \ldots \ldots \ldots \ldots$

6.8. Contour plot of maximum temperature in segregation volume on $\mathrm{G} 693$ fuel plates drawn to scale, first time step of LAT analysis. Shaded region exceeds $400^{\circ} \mathrm{C} \ldots \ldots 60$

6.9. Contours of temperature for spot that has the highest maximum temperature of the G693 core design, nominal LAT analysis 
6.10. Carpet plot of maximum temperatures in region near the segregatiuon volume. Region

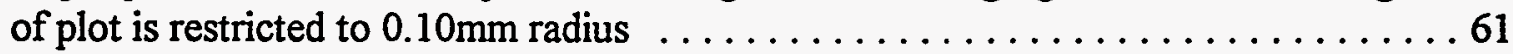

6.11. Maximum temeprature in normal fuel as a function of time for the G693 core design,

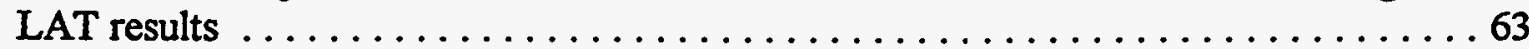

7.1. DART Thermal conductivity estimates .....................67

7.2. Thermal conductivity for pure $\mathrm{U}_{3} \mathrm{Si}_{2}$ and $\mathrm{U}(\mathrm{A1}, \mathrm{Si})_{3} \ldots \ldots \ldots \ldots \ldots \ldots 6$

7.3. Maximum temperatures in a $\mathrm{G} 693 \mathrm{LAT}$ analysis with conversion of fuel to $\mathrm{U}(\mathrm{Al}, \mathrm{Si})_{3} \quad .68$

7.4. Carpet plot of termpatures aroun hot spot with highest temeprature. G693 LAT results with fuel conversion to $\mathrm{U}(\mathrm{Al}, \mathrm{Si})_{3} \ldots \ldots \ldots \ldots \ldots \ldots \ldots \ldots \ldots$

7.5. Maximum temperatures from G693 LAT anlaysis using different transition

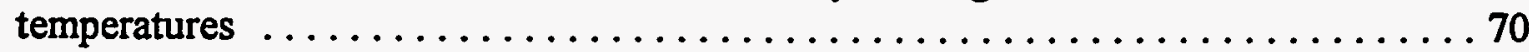

7.6. Maximum temperature for G693 LAT analyses suing standard 2-mm and 1-mm-diam.

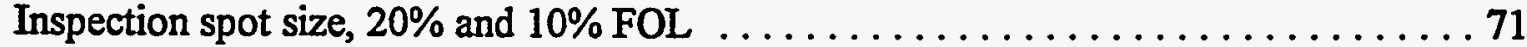

7.7. Maximum peaking factors for G693 LAT analyses using standard $20 \mathrm{~mm}$ and $1-\mathrm{mm}-$ diam. Inspection spot size, $20 \%$ and $10 \%$ FOL $\ldots \ldots \ldots \ldots \ldots \ldots \ldots \ldots \ldots$

7.8. Maximum temperature for $\mathrm{G} 693 \mathrm{~L}$ Lat analyses using standard $20 \mathrm{~mm}$-diam. Inspection spot size, $10 \%$ FO with different transition temepratures .............. 72

7.9. HEATING maximum temperatures for the $\mathrm{G} 693,300 \mathrm{MW}$, zero uncertainties case. Comparison of adiabatic and perfect thermal contact models with models using air in nonbond cavity ................................ 74

8.1. Thermal conductivity estimates for high-enriched and medium-enriched normal fuel . . 75

8.2. Thermal conductivity estimates for highly-enriched and medium-enriched segregated

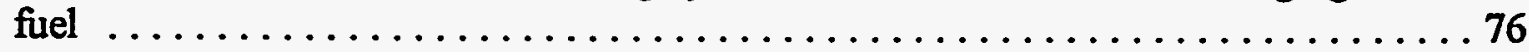

8.3. Comaprison of temperatures from Lat anlaysis, $95 \%$ limits, G693, high-enriched and

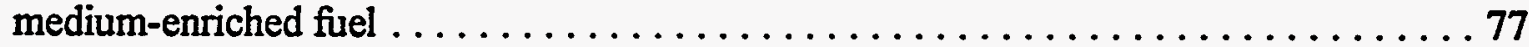

8.4. Comparison of maximum peaking factors from LAT analysis, $95 \%$ limits, G693, high0enriched and medium-enriched fuel $\ldots \ldots \ldots \ldots \ldots \ldots \ldots \ldots \ldots . \ldots \ldots 77$

8.5. Maximum temperatures and peaking factors as a function of diameeter of theexcess fuel loading inspection spot. Fuel overload $-20 \% \ldots \ldots \ldots \ldots \ldots \ldots \ldots 79$

8.6. Maximum temperatures and peaking factors as a function of the excess fuel loading overload factor. Segregation inspection spot diameter $-0.54 \mathrm{~mm} \ldots \ldots \ldots . .79$

8.7. DART thermal conductivity estimates for medium-enriched normal fuel as a function

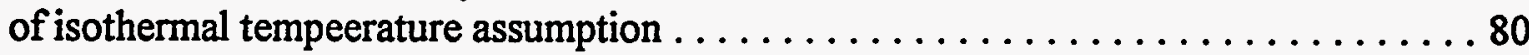

8.8. Comparison of maximum temepratures for LAT analyses of the modified G693, $95 \%$ limits case using DART produced thermal conductivities of the normal fuel with different isothermal temperature assumptions $\ldots \ldots \ldots \ldots \ldots \ldots \ldots \ldots \ldots . .61$

B.1. Critical heat flux anlaysis model $\ldots \ldots \ldots \ldots \ldots \ldots \ldots \ldots \ldots \ldots \ldots . \ldots \ldots \ldots \ldots$

B.2. Maximum peaking factor as a function of the size of an adiabatic region on the surface on the model $\ldots \ldots \ldots \ldots \ldots \ldots \ldots \ldots \ldots \ldots \ldots \ldots \ldots \ldots \ldots . . . . \ldots \ldots$

B.3. Maximum temperature in the center of segregation volume as a function of the size of an adiabatic region on the surface on the model $\ldots \ldots \ldots \ldots \ldots \ldots \ldots \ldots .4$ 


\section{LIST OF FIGURES (continued)}

C.1. Peaking factor correlation fro permutatin data set compared wiht L7-best data ..... C.3

C.2. Maximum temperature correlation of permutation data set compared with L7-best dataC.6

D.1. Meat thickness distribution for the $\mathrm{L} 7$ core design $\ldots \ldots \ldots \ldots \ldots \ldots \ldots \ldots \ldots \ldots$

D.2. Relative power density distribution on $\mathrm{L} 7$ plates, all time steps ........... D. 4

D.3. Contours of thermal conductivity of normal fuel for each time step, L7 core design . . D.5

D.4. Contours of thermal conductivity of segregated fuel, $\mathrm{L} 7$ design $\ldots \ldots \ldots \ldots \ldots$ D. 6

D.5. Contours of fission density, L7 design, $95 \%$ limits TASHA case ........... D.7

D.6. contours of oxide layer thickness, "Best Estimate" TASHA case, L7 design . . . . . D.8

D.7. Contours of oxide layer thickness, "Best Estimate" TASHA case, L7 design . . . . . D.9

D.8. Contours of coolant bulk temperature, "Best Estimate" TASHa case, L7 design . . . . D.10

D.9. Meat thickness distribution for the $\mathrm{L} 7$ core design $\ldots \ldots \ldots \ldots \ldots \ldots \ldots \ldots \ldots \ldots \ldots \ldots$

D.10. Contours of relative power density for each time step, G693 ........... D. 14

D.11. Contours of thermal conductivity of normal fuel, G693 design ........... D.15

D.12. Contours of thermal conductivity of segregated fuel, G693 $\ldots \ldots \ldots \ldots \ldots \ldots$ D. 16

D.13. Contours of fission density, G693 . . . . . . . . . . . . . . . . . . . D.17

D.14. Contours of oxide layer thickness, "Best Estimate" TASHA case, G693 . . . . . . . D. D.18

D.15. Contours of coolnat temperature, "Best Estimate" TASHA case, G693 . . . . . . . D.19

E.1. Comparison of fission density generated from power density (shaded) and burnup data

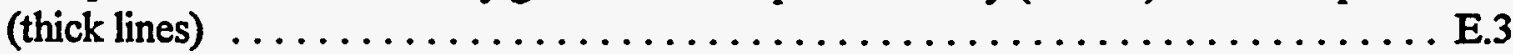

E.2. Relative difference between fission density generated from power density and burnup data 


\section{LIST OF TABLES}

Table

Page

1.1. ANSR fuel plate dimensions for hot spot investigations $\ldots \ldots \ldots \ldots \ldots \ldots \ldots$

2.1 Thermal properties for Hilvety and Chapman study $\ldots \ldots \ldots \ldots \ldots \ldots \ldots$

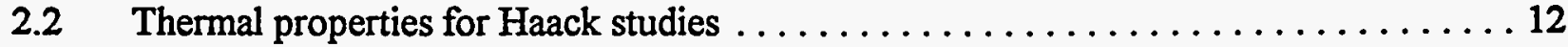

2.3 Thermal properties for Kirkpatrick ANSR studies $\ldots \ldots \ldots \ldots \ldots \ldots \ldots \ldots$

3.1 Fuel Thermal Conductivities . . . . . . . . . . . . . . . . . . . . 17

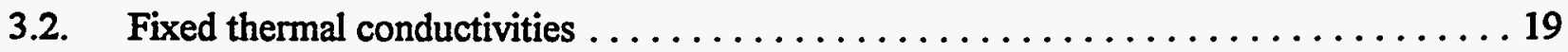

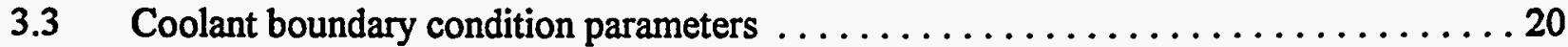

3.4. ANSR Thermal design limits pertinent to hot spot investigations $\ldots \ldots \ldots \ldots \ldots$

4.5 Summary of $\mathrm{L} 7$ core design values at EOC $\ldots \ldots \ldots \ldots \ldots \ldots \ldots \ldots \ldots$

A.1 Format of the input meat thickness distribution data set $\ldots \ldots \ldots \ldots \ldots \ldots \ldots$ A.2

A.2 Format of a time step block in the relative power density data set . . . . . . . . A.3

A.3 Format of a time-step block in the oxide thickness data set . . . . . . . . . A.3

A.4 Format of a time step block in the coolant temperature data set $\ldots \ldots \ldots \ldots \ldots$. . . .

A.5 Format of a time-step block in the Fission Density Data set $\ldots \ldots \ldots \ldots \ldots \ldots$. . . . . .

A.6 Format of tecconl and tecconu files $\ldots \ldots \ldots \ldots \ldots \ldots \ldots \ldots \ldots \ldots \ldots$

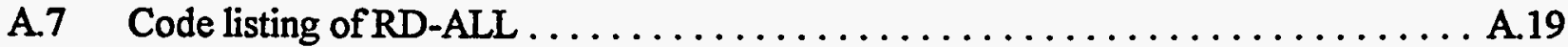

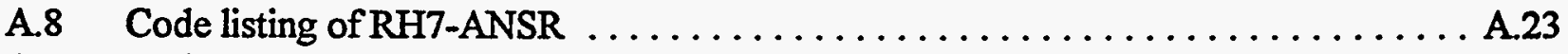

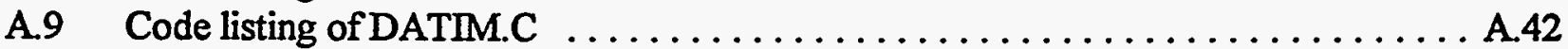

C.1 Ranges of parameters $\ldots \ldots \ldots \ldots \ldots \ldots \ldots \ldots \ldots \ldots \ldots \ldots \ldots \ldots \ldots \ldots$. 2

C.2 Fit quality of peaking factor correlation for various data sets $\ldots \ldots \ldots \ldots \ldots \ldots$. $\ldots \ldots$

C.3 Fit quality of maximum temperature correlation for various data sets . . . . . . . C.8

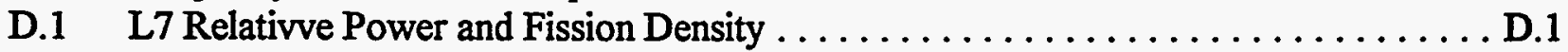

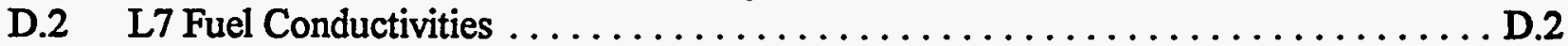

D.3 L7 TASHA 95\% Limits Case Oxide Thickness and Coolant Temeprature . . . . . . . D.2

D.4 L7 TASHA "best estimates" Case Oxide Thickness and Coolnat Temeprature . . . . . D.2

D.5 G693 Relative Power and Fission Density . . . . . . . . . . . . . . . . . . D.3

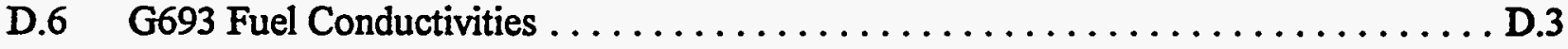

D.7 G693 TASHA 95\% Limits Case Oxide Thickness and Coolant Temperature . . . . . . D.4

D.8 G693 TASHA "best estimates" Case Oxide Thickness and Coolant Temperature . . . . D.4

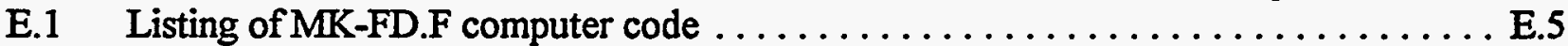

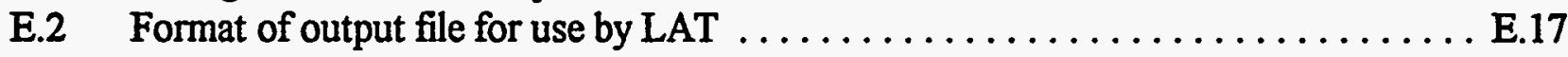

E.3 Format of output file for surface density distribution $\ldots \ldots \ldots \ldots \ldots \ldots \ldots \ldots$

E.4 Format of input file for burnup fraction distribution $\ldots \ldots \ldots \ldots \ldots \ldots \ldots \ldots$

E.5 Format of output file for use with TECPLOT $\ldots \ldots \ldots \ldots \ldots \ldots \ldots \ldots \ldots$ 


\section{ACRONYMS}

\begin{tabular}{|c|c|}
\hline $1-\mathrm{D}$ & one-dimensional \\
\hline 2-D & two-dimensional \\
\hline 3-D & three-dimensional \\
\hline ANS & Advanced Neutron Source \\
\hline ANSR & Advanced Neutron Source reactor \\
\hline $\mathrm{BOC}$ & beginning-of-cycle \\
\hline CHF & critical heat flux \\
\hline DART & dispersion analysis research tool \\
\hline DNB & departure from nucleate boiling \\
\hline $\mathrm{EOC}$ & end-of-cycle \\
\hline FO & fuel overload factor \\
\hline HEU & High Enriched Uranium \\
\hline HFIR & High Flux Isotope Reactor \\
\hline LAT & local analysis technique \\
\hline LSSS & limiting safety system set points \\
\hline NA & not applicable \\
\hline ORNL & Oak Ridge National Laboratory \\
\hline THTL & Thermal Analysis of Steady-State Heat Transfer for ANS \\
\hline
\end{tabular}




\section{ACKNOWLEDGMENTS}

My thanks to Grady Yoder, Dave Morris and the ANSR project for sponsoring my efforts for the several years of our association. George Copeland has provided many instructive discussions and insights and has reviewed the document in various forms. Jeff Rest has been gracious to supply thermal conductivity estimates in the forms needed for this effort. Also thanks to John Kirkpatrick for the many discussions of aspects of the HFIR and ANSR.

A special thanks to Colin West, Doug Selby and Paul Williams who were gracious enough to spend the time and attention to greatly increase the readability and accuracy of this report. Of course, our technical editor, Cathy Shappert, also greatly improved the readability and understanding of this report. 



\section{ABSTRACT}

The Advanced Neutron Source Reactor (ANSR) is a research reactor designed to provide the highest continuous neutron beam intensity of any reactor in the world. The present technology for determining safe operations were developed for the High Flux Isotope Reactor (HFIR). These techniques are conservative and provide confidence in the safe operation of HFIR. However, the more intense requirements of ANSR necessitate the development of more accurate, but still conservative, techniques. This report details the development of a Local Analysis Technique (LAT) that provides an appropriate approach.

Application of the LAT to two ANSR core designs are presented. New theories of the thermal and nuclear behavior of the $\mathrm{U}_{3} \mathrm{Si}_{2}$ fuel are utilized. The implications of lower fuel enrichment and of modifying the inspection procedures are also discussed.

Development of the computer codes that enable the automatic execution of the LAT is included. 


\section{INTRODUCTION}

The ANSR is a research reactor designed to provide the highest continuous neutron beam intensity of any reactor in the world. Additional objectives of the design are to provide materials irradiation facilities and isotope production facilities as good as or better than the High Flux Isotope Reactor (HFIR). Thus the operational conditions of this design are more challenging than in HFIR.

\subsection{PURPOSE OF STUDY}

The techniques used to provide safety analyses of the HFIR fuel plate defects provided conservative estimates of the maximum peaking factors and fuel meat temperatures. The peaking factor is the ratio of the local surface heat flux at a fuel defect location to the nominal heat flux (assuming homogenous fuel at design conditions) at that location. These techniques were sufficient to provide confidence in the safe operation of the HFIR design. However, because the ANSR operating conditions are more demanding than in HFIR, these techniques are too limiting. The overly conservative analysis assumptions would require reducing maximum power to levels that would not meet experimental needs.

This report describes an analysis technique that provides a more accurate, but still conservative, estimate of the maximum peaking factors and fuel meat temperatures caused by fuel defects and other perturbations from the design conditions.

The defects and perturbations investigated include: local fuel segregation, lack of a metallurgical bond, local coolant temperature, thermal physical property variation due to composition, temperature, and fission density, and location in the core.

\subsection{ANSR FUEL PLATE DESIGN}

The ANSR fuel is contained in thin composite plates that are manufactured in an involute shape. The involute plates are placed in a cylindrical volume with coolant channels between adjacent plates (Figs. 1.1 and 1.2). This design maintains constant thickness coolant flow channels and fuel plates across the cylindrical volume. The fuel plate is composed of clad layers that are of equal thickness, with a central volume that contains filler and/or fuel meat. The edges of the plate (in the span direction) contain no fuel meat, instead the central volume in the edge region is filled with the same material as the clad. Thus the fuel is sealed into the plate on both sides and along the edges by the clad material, Aluminum 6061 . The clad, filler and meat are metallurgically bonded by a rolling process that compresses the 
fuel meat into a cermet and produces a plate of the required thickness and uniformity.

The fuel is distributed in a configuration to level power throughout the core. In the HFIR design this is accomplished by a radial grading or variation of meat thickness as a function of radius to control the local fuel loading. The ANSR design requires both an axial and a radial variation.

The normal fuel meat is a powder mixture of $\mathrm{U}_{3} \mathrm{Si}_{2}(11-30 \%$ by volume) and aluminum. The meat may not fill the central volume. The rest of the central volume is filled with aluminum powder. Table 1.1 contains the geometric parameters of the ANSR fuel plate that are pertinent to the hot spot studies.

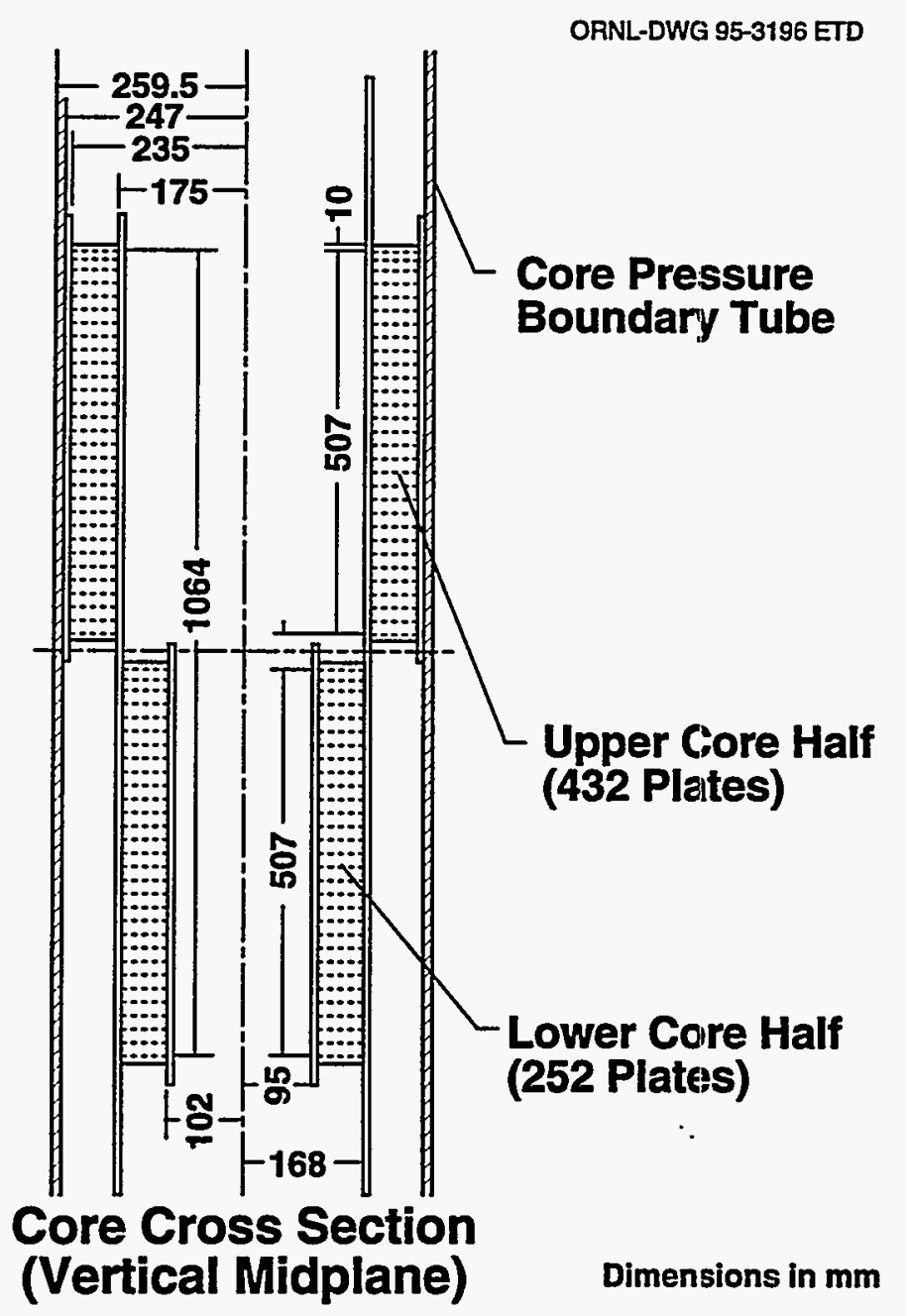

Fig. 1.1. ANSR fuel elements: vertical midplane section.

The fuel meat in the HFIR design is located next to one of the clad layers, producing an offset fuel layer when the fuel layer thickness is less than maximum. The proposed ANSR design requires that the fuel meat be 
centered in the fuel volume. Figure 1.3 compares these two designs for the minimum fuel meat thickness condition.

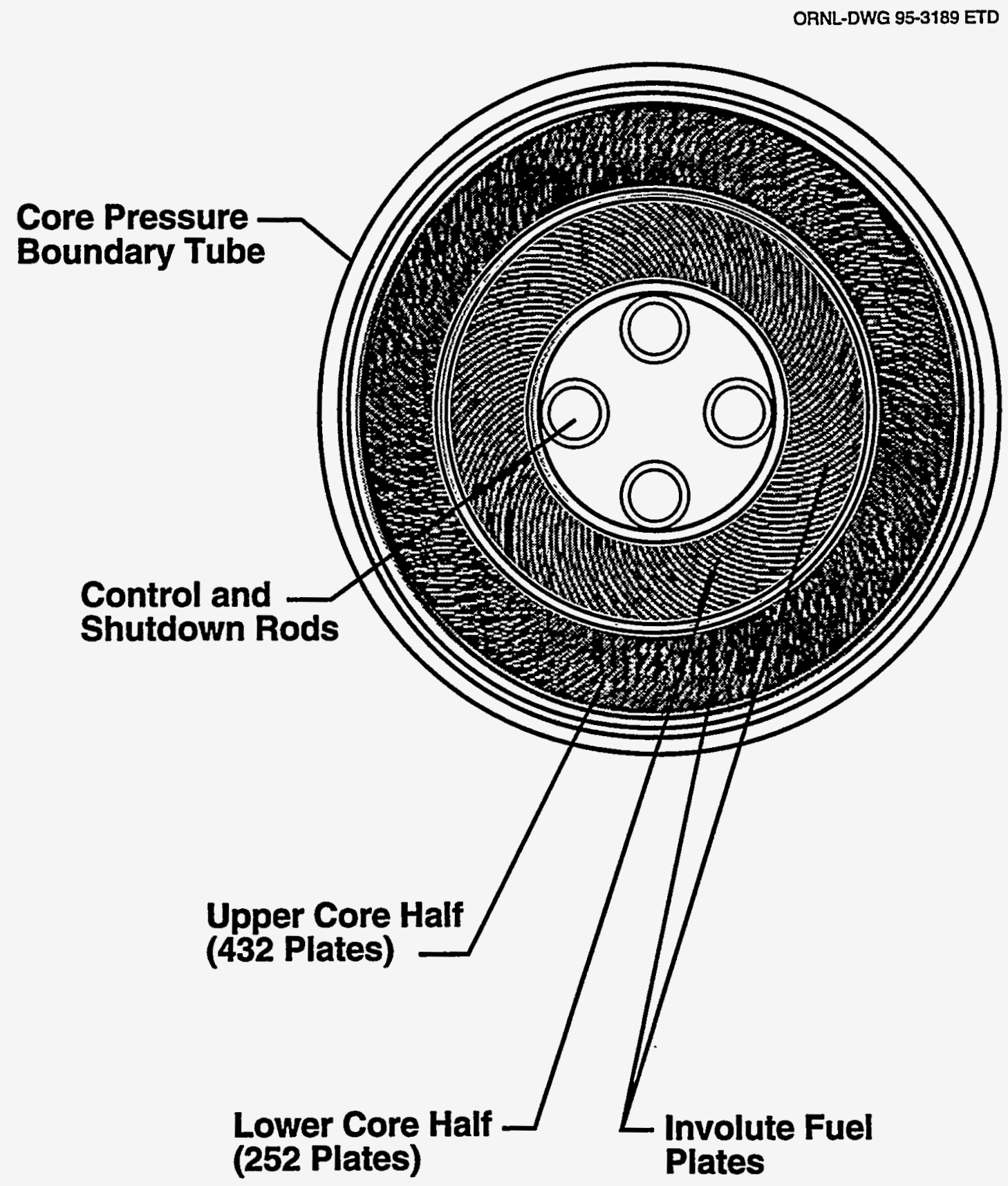

Plan View Dimensions in $\mathrm{mm}$

Fig. 1.2. Horizontal section of the fuel elements. 
Table 1.1 ANSR fuel plate dimensions for hot spot investigations

\begin{tabular}{llr}
\hline Clad thickness & $0.254 \mathrm{~mm}$ & $(10 \mathrm{mil})$ \\
Central volume thickness & $0.762 \mathrm{~mm}$ & $(30 \mathrm{mil})$ \\
Design maximum fuel thickness & $0.762 \mathrm{~mm}$ & $(30 \mathrm{mil})$ \\
Design minimum fuel thickness & $0.1778 \mathrm{~mm}$ & $(7 \mathrm{mil})$ \\
Total plate thickness & $1.270 \mathrm{~mm}$ & (50 mil) \\
\hline
\end{tabular}

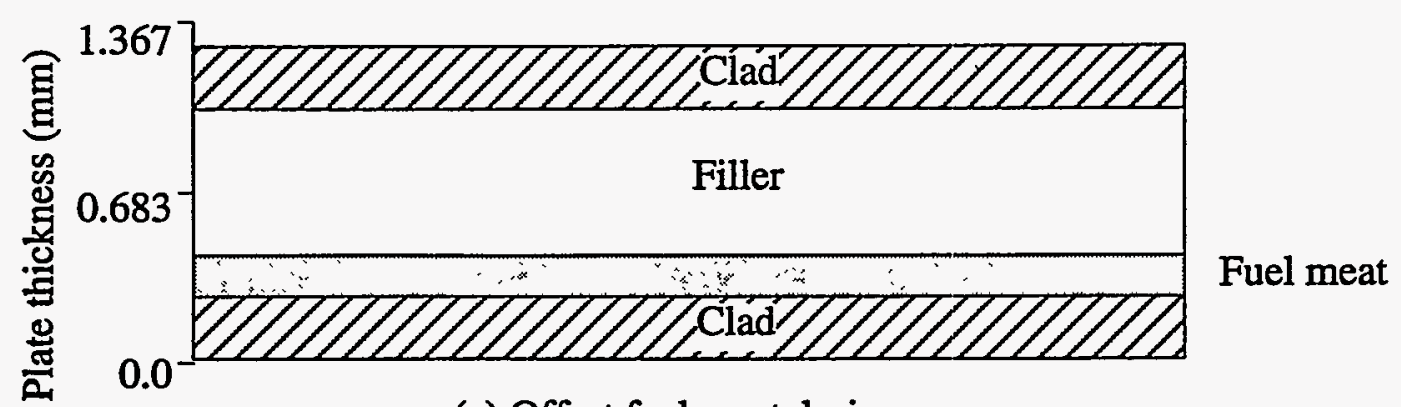

(a) Offset fuel meat design

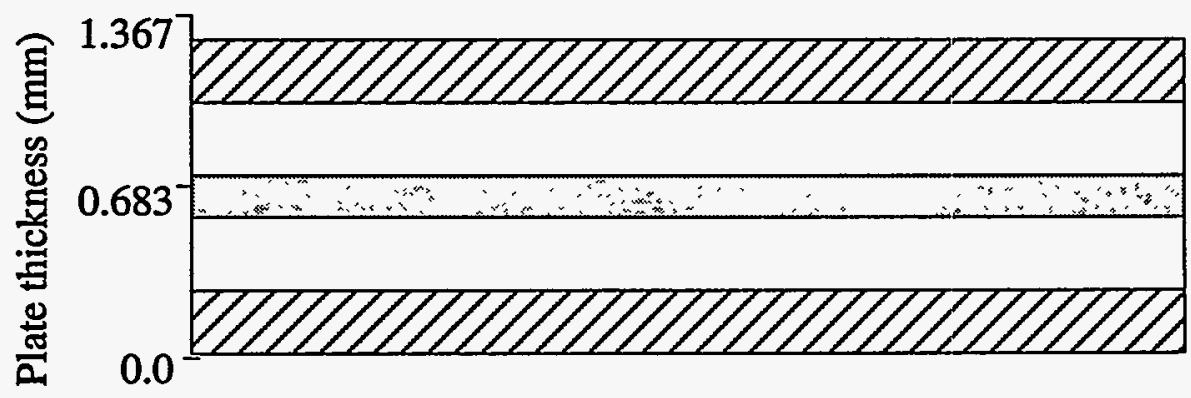

(b) Centered fuel meat design

Fig 1.3. Section of the ANSR fuel plate showing placement of fuel meat within fuel volume for (a) offset and (b) centered fiel meat design.

\subsection{FUEL TEMPERATURE CRITERION}

The current ANSR criterion for the maximum temperature in the fuel was set in response to some of the studies described in this report. The original criterion requires that the fuel temperature not exceed $400^{\circ} \mathrm{C}$ for even the smallest volume. As discussed in later sections the application of this criterion to minuscule "hot spot" proved to be overly conservative and might be excessively restrictive on the reactor performance. A new criterion for consideration was proposed by the ANSR Fuel Temperature Workshop also described later in this report. This criterion restricts the average temperature in a small fuel volume to be less than $400^{\circ} \mathrm{C}$. The volume is 
calculated for a cylinder whose height is the thickness of the fuel meat layer and whose cross-sectional area is $1.0 \times 10^{-6} \mathrm{~m}^{2}$.

\subsection{FUEL DEFECTS STUDIED}

\subsubsection{Excess Local Fuel Loading}

The fuel meat is composed of solid fuel particles in an aluminum matrix with some voids possible. The fuel is manufactured by mixing aluminum powder with fuel particles. Particle size control is achieved by passing the meat and filler mixtures through several screens to limit the maximum size of an individual particle in the mixture to less than $0.015 \mathrm{~mm}$ (150 microns). However, the mixing of the fuel particles and filler material can produce inhomogeneities, regions of excess fuel that are called segregation spots. It is postulated that several fuel particles somehow agglomerate into volumes larger than the maximum single particle size. The maximum concentration expected in this segregated material is estimated to be $50 \% \mathrm{U}_{3} \mathrm{Si}_{2}$ with $35 \%$ aluminum and $15 \%$ void. ${ }^{1}$ Volumes of this material in the fuel plate are detectable in the X-ray inspection process if there is greater than a $20 \%$ fuel overload in a 2-mm-diameter spot. The $20 \%$ excess fuel is called the fuel overload factor (FO). A plate with one such spot is rejected as unacceptable.

The size and shape of the local fuel segregation volume and the concentration of the segregated material within the 2-mm-diam spot are unknown. As a worst-case approximation, a volume that contains all the excess fuel is assumed to be at the center of the inspection spot. A cylinder of the maximum concentration of fuel that produces the uniformly distributed FO in the inspection spot size is assumed to be the segregation volume. The cylinder height is assumed to be the same as the meat layer thickness. The cylinder geometry produces the highest surface heat flux estimates and is therefore a conservative assumption. The radius of the cylinder is a function of the FO, inspection spot size, maximum concentration and maximum fuel loading.

\subsubsection{Lack Of Metallurgical Bond}

Another potential manufacturing flaw is a failure to form a metallurgical bond (nonbond) in the plate during the rolling process. Proposed inspection procedures will limit the maximum allowable nonbond to a $1-\mathrm{mm}$ diameter.

This nonbond area can produce a major modification to the local heat flow and increase the local heat flux from the surface of the clad into the coolant channel at the opposite side of the plate. The heat transfer across a nonbond depends on many parameters such as contact pressure and gas 
composition between the surfaces of the nonbond, fuel composition at the surfaces, hardness, yield strength, fuel swelling, local burnup, and surface emissivities. Most of these parameters are not known with any precision for the conditions in the ANSR. Although there will be some heat transfer across the nonbond, an adiabatic assumption (no heat transfer) will result in a higher local heat flux and maximum temperature and is therefore conservative. With this assumption the exact thickness assumed for the nonbond is not vital as long as the thickness is a small fraction of the diameter. In most of the analyses of this study, the nonbond thickness is assumed to be $0.1 \mathrm{~mm}(100 \mu \mathrm{m})$.

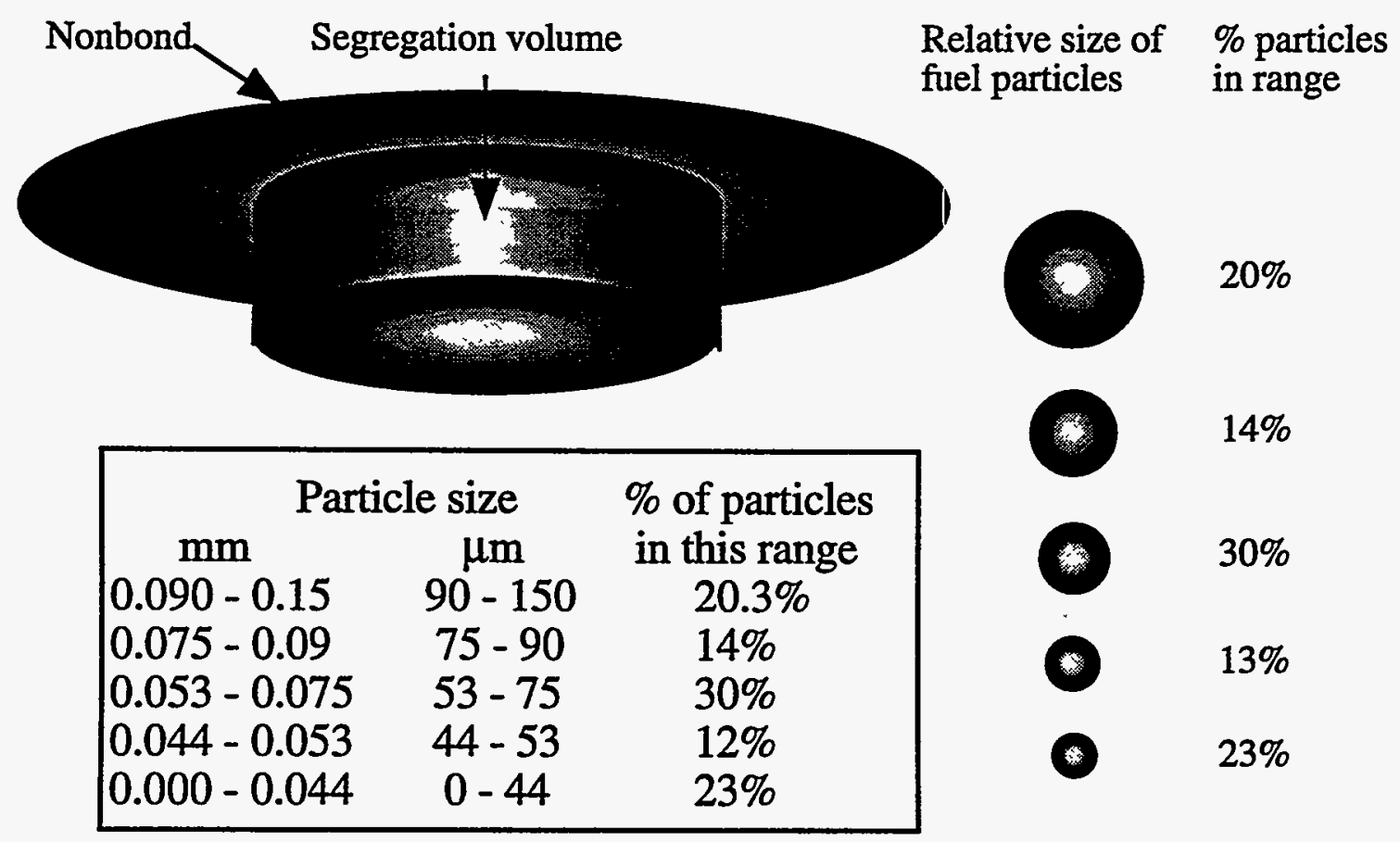

Sizes of segregation volume and fuel particles

Minimum meat thickness

Maximum fuel particle size $\frac{\mathrm{mm}}{0.178} \quad \frac{\mu \mathrm{m}}{178}$

Segregation cylinder diameter

0.15

150

(For $50 \% \mathrm{U} 3 \mathrm{si} 2,35 \% \mathrm{Al}, 15 \%$ void)

0.512 . 512

Fig. 1.4. Segregation volume and nonbond compared with fuel particle size range.

The most severe position for a nonbond depends upon the type of plate design. For an offset or a perfectly centered neat layer the most-severe location is on the fuel meat and filler interface. This arrangement produces 
the maximum peaking factor and fuel temperature. In investigations of fuel meat layers that are slightly displaced from the exact center due to manufacturing tolerances, the most damaging nonbond void position is on the meat-filler interface that is closest to the center of the plate.

\subsubsection{Centering Tolerance}

The ANSR design requires that the fuel meat layer be centered in the volume between the clad layers. The extent to which manufacturing processes will be able to achieve this goal has not been established. Small variations in the meat layer location could make significant differences in the peaking factors.

\subsubsection{Coolant Temperature}

In the previous hot spot studies (discussed in Sects. 2 and 3), the coolant bulk temperature was conservatively assumed to be the average exit coolant temperature. Most of the plate coolant is significantly lower in temperature. The lower local coolant temperatures will lower the local maximum fuel temperatures. Also, because most material properties and the forced convection heat transfer coefficient are temperature dependent, the peaking factors could be affected.

The use of the average exit temperature as the local coolant temperature also neglects the possibility of the upstream coolant being heated to higher than the exit temperature by a sequence of hot spots or other phenomena. This effect is approximated in the detailed or Local Analysis Technique (LAT) because the coolant temperatures are obtained from a TASHA analysis that incorporated the hot streak factors.

\subsubsection{Oxide Layer Thickness}

The oxide layer that grows on the aluminum-clad surface in contact with the coolant restricts the flow of heat to the coolant and can reduce the flow area of the channel. The temperature difference across the oxide layer is included in the LAT by modifying the heat transfer coefficient. The reduced flow area effect is incorporated into the LAT for some analyses by using the TASHA-generated coolant temperatures and by adjusting the heat transfer coefficient to that of the minimum thickness channel.

\subsection{ORGANIZATION OF REPORT}

A discussion of the previous studies of HFIR and ANSR fuel plate defects is included in Sect. 2. Analyses performed by Giles for the ANSR are discussed in Sect. 3 as a background for the LAT developed for the ANSR that is discussed in Sect. 4. Section 5 discusses the LAT analyses of 
the L7 core design while Sect. 6 discusses the same for the G693 core design. The ANSR "Workshop on Maximum Fuel Temperatures" is discussed in Sect. 7. Section 7 also contains a description of the extension of the LAT to incorporate these latest theories on the changes to the fuel induced by the nuclear and thermal conditions. Section 8 contains the description of the application of the LAT to medium, and low-enriched fuels and to the three-element core design.

The technique that allows automatic execution of the LAT is discussed in Appendix A, including a listing of the FORTRAN code, RH7-ANSR. Appendix B details a limited investigation of the growth potential of a steam bubble that might form on the surface of the fuel plate. Appendix $\mathrm{C}$ reports on the development of a functional replacement for the LAT, that under certain circumstances, might allow a single function to conservatively replace the more detailed and complex LAT. Appendix D reports on the details of the L7 core design. Appendix E contains a detailed description of the method of estimating the fission density as a function of time and compares this method with the VENTURE produced data at the end of the cycle. 


\section{PREVIOUS ANALYSIS OF FUEL DEFECTS}

\subsection{HFIR}

The ANSR fuel plate design is similar to the HFIR fuel plate. HFIR uses the same clad and filler material as ANSR but with uranium oxide fuel particles. The clad, fuel volume, overall plate and coolant channel thickness for both reactor designs are the same. HFIR uses a fuel meat design that is offset or adjacent to one of the clad plates. The general size of the fuel plates is similar for both reactors. The fuel loading and distributions and the location of the fuel plates are different, however.

On the scale of the fuel defects, the fuel plates are very similar and therefore the previous HFIR studies are pertinent to the ANSR.

\subsubsection{Hilvety and Chapman² Study (ORNL-TM-1903, 1967)}

This study of the HFIR fuel element has been one basis for the safety analyses for the HFIR reactor. A portion of this study involved the determination of the effect of potential fuel defects. These defects include the lack of a metallurgical bond and a coincident local fuel segregation volume. This defect pair produces local modifications to the surface heat flux that are bounded by the uncertainty factors. These uncertainty factors are used in the HFIR Steady State Thermal-Hydraulic code to determine operational limits and control and safety system set points.

The model developed for the hot spot study was a two-dimensional (2-D) axisymmetric heat transfer model $(12.7-\mathrm{mm}$ radius) around a coincident nonbond and segregation volume (Fig. 2.1). Thus the segregation volume and the nonbond were cylindrical features. Limiting the model to a small region around the defect allowed the calculation of maximum temperatures in the segregated fuel and detailed heat flux distributions on the surface of the clad to the coolant. The outer radius of the model was assumed to be adiabatic.

The nonbond was assumed to be a perfectly insulating thin $1.588-\mathrm{mm}$ (0.0625-in.) diameter disk. This disk was centered over the segregation spot at the junction of the meat layer and the filler material. The thickness of this disk was not reported.

The maximum excess fuel loading for this study was $30 \%$ in a 1.98-mm (5/64-in.)-diameter inspection area. The segregation volume was assumed to extend completely across the meat layer and was assumed to be comprised of closely packed uniform spheres of $\mathrm{U}_{3} \mathrm{O}_{8}$ (packing factor, 0.74). The cylinder radius was $0.208 \mathrm{~mm}(0.0082 \mathrm{in}$.). The heat generation in this 
cylinder due to the more concentrated fuel is 10.3 times that of normal fuel in the same location. The heat generation for the segregated material appears to be overly conservative. It is roughly twice the maximum possible. ${ }^{3} \mathrm{~A}$ smaller heat generation of $270 \mathrm{~W} / \mathrm{cm}^{3}\left(15,100 \mathrm{Btu} / \mathrm{h} \cdot \mathrm{in}^{3}\right)$ was assumed to exist in the clad.

The thermal properties of the materials were assumed to be constant and are reported in Table 2.1. These properties represent the materials at the beginning-of-cycle or unirradiated condition.

Table 2.1 Thermal properties for Hilvety and Chapman study

\begin{tabular}{lcc}
\hline & \multicolumn{2}{c}{ Conductivity } \\
\cline { 2 - 3 } Material & $\mathrm{W} / \mathrm{m}^{\circ} \mathrm{C}$ & Btu/h.in..$^{\circ} \mathrm{F}$ \\
\hline Normal Fuel $\mathrm{U}_{3} \mathrm{O}_{8}+\mathrm{Al}$ & 168.5 & 97 \\
Segregated Fuel $\mathrm{U}_{3} \mathrm{O}_{8}$ & 47.1 & 27.1 \\
Clad Al & 168.5 & 97 \\
\hline
\end{tabular}

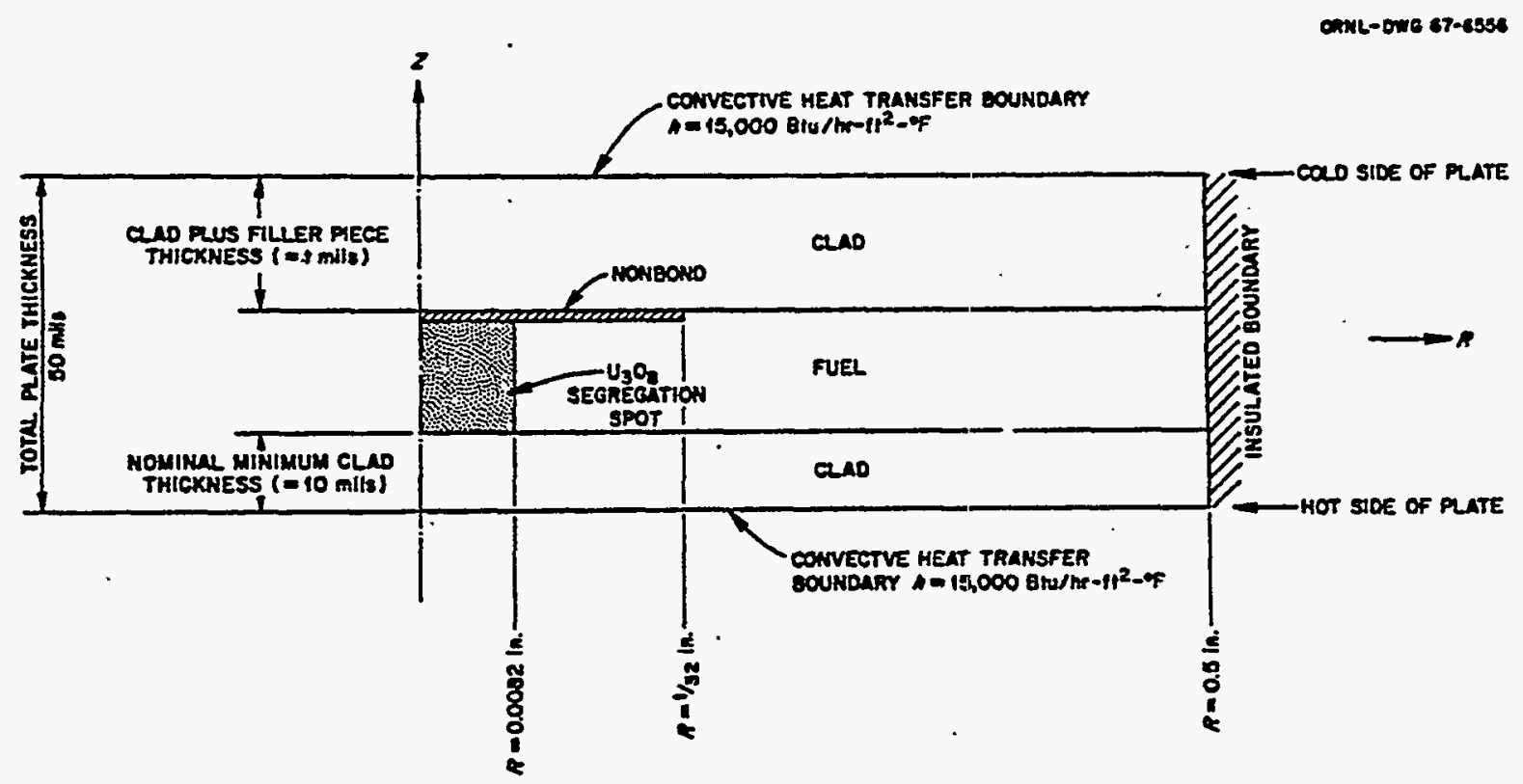

Basic ASSUMPTIONS:

1. NO HEAT TRANSFER ACROSS NON-BOND

2. CIREUNAFEREHTIAL STMMETRY

3. PACXING FRLCTION OF UJOA PARTICLES UN SEGREGATION SPOT $=0.74$

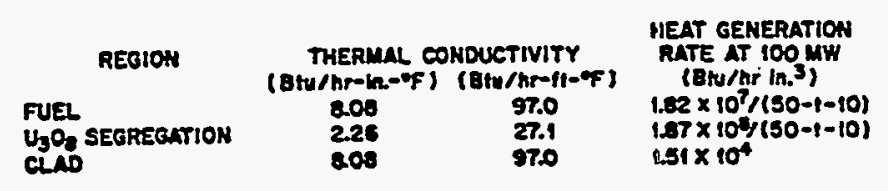

Fig. 2.1. Hilvety and Chapman thermal model. 
Although an oxide layer is mentioned in the report, neither the thickness used nor the conductivity of the oxide film is reported. A fixed, forced convection heat transfer coefficient was applied to the upper and lower clad surfaces of $85 \mathrm{~kW} / \mathrm{m}^{2}{ }^{\circ} \mathrm{C}\left(15,000 \mathrm{Btu} / \mathrm{h} \cdot \mathrm{ft}^{2} .^{\circ} \mathrm{F}\right)$. The fluid temperature used for this boundary condition was not reported.

The conservative estimate for the maximum peaking factor determined by Hilvety and Chapman was 1.7.

\subsubsection{Haack ${ }^{4}$ Analyses (ORNL-TM-1904, 1967)}

This study reviewed the previous work using more recent information about material properties and inspection techniques and concluded that previous uncertainty factors were conservative for isolated coincident nonbond/segregation spots. The basic axisymmetric model reported in ref. 4 was similar to ref. 2 except as noted below.

The nonbond was modeled by an adiabatic disk the same size as in ref. 2 with a thickness of $0.0254 \mathrm{~mm}$ (0.001 in.).

The segregation cylinder radius was increased to $0.249 \mathrm{~mm}$ (0.0098 in.). The maximum excess fuel loading allowed in this study was reduced to $27 \%$ in a $1.98-\mathrm{mm}$ (5/64-in.)-diameter inspection spot. The exact values of heat generation rates were not reported but described as follows:

The heat generation rates in the nominal fuel were determined from the power density data assuming that all of the heat was generated in the fuel cermet. Those in the segregated fuel were assumed to be those in the nominal fuel multiplied by the ratio of the packing factor of the segregated fuel to that of the nominal fuel.

The clad was assumed to generate heat at a rate of $100 \mathrm{~W} / \mathrm{g}$ that is equivalent to the $270 \mathrm{~W} / \mathrm{cm}^{3}$ of ref. 2 . This heat generation in the clad appears to be a conservative addition of heat load to the model.

The thermal properties of the materials were assumed to be constant and are reported in Table 2.2.

The maximum oxide layer thickness was assumed to be $0.076 \mathrm{~mm}$ ( 0.003 in.). The same heat transfer coefficient as in ref. 2 was applied to the clad surfaces. The fluid temperature was not reported.

Haack's studies were reported in slightly more detail and investigated effects such as the size of the axisymmetric model and the segregation cylinder and the presence of adjacent defects or a second coincident nonbond. Haack also studied the area near the edge of the plate where the meat layer is thinnest and power density the highest. 
Although Haack did not duplicate the Hilvety and Chapman analysis exactly, he concluded that the previous results were conservative for isolated defect pairs (coincident nonbond and segregation cylinder).

Table 2.2 Thermal properties for Haack studies.

\begin{tabular}{lcc}
\hline & \multicolumn{2}{c}{ Conductivity } \\
\cline { 2 - 3 } Material & $\mathrm{W} / \mathrm{m}^{\circ} \mathrm{C}$ & $\mathrm{Btu} / \mathrm{h} \cdot \mathrm{in} \cdot 0^{\circ} \mathrm{F}$ \\
\hline Fuel Inner Element & 132.5 & 76.3 \\
Fuel Outer Element & 110.5 & 63.6 \\
Segregated Fuel $\mathrm{U}_{3} \mathrm{O}_{8}$ & 13.05 & 7.51 \\
Clad & 151.1 & 97.0 \\
Oxide & 2.25 & 1.3 \\
\hline
\end{tabular}

\subsubsection{Kirkpatrick ${ }^{3}$ HFIR Analyses (K/CSD/TM-79, 1990)}

This study reviewed Hilvety and Chapman's work again using modern computer codes. Where possible, Kirkpatrick's major assumptions were the same as Hilvety and Chapman to verify the original analyses. He also investigated variations in many parameters that were not reported in the original documents and investigated the effects of alternative assumptions on segregation spot models (power and size), nonbonds (size, location and heat transfer), and reactor power. Again Kirkpatrick could not duplicate the analyses in ref. 2 exactly but concluded that previous hot side peaking factors were conservative.

\subsection{ANSR}

\subsubsection{Kirkpatrick5,6 ANSR Analysis (Internal Correspondence, 1989)}

Kirkpatrick used axisymmetric hot spot models and assumptions similar to the previous HFIR studies to estimate the uncertainty factors for the ANSR fuel plate design. The effects of variation in reactor power, thermal conductivities, nonbond size, and oxide layer thickness were performed. Coincident and noncoincident (or independent) nonbonds and segregation cylinders were also studied.

The basic model was the same as ref. 2 with the following changes to some of the modeling parameters. The nonbond diameter was the same as previous studies but the thickness was $0.001 \mathrm{~mm}\left(3.94 \times 10^{-5}\right.$ in.). This is smaller than reported by Haack. The thickness of a physical nonbond is not known. The effect of the nonbond thickness on peaking factor (the major result of these and previous studies) is not great as long as the thickness is a 
small faction of the clad. The maximum uranium density was varied from 1.6 to $2.25 \mathrm{mg} / \mathrm{mm}^{3}$ in this study. The thermal parameters used in this study are reported in Table 2.3 .

The segregation cylinder was assumed to be formed of pure solid fuel $\left(\mathrm{U}_{3} \mathrm{Si}_{2}\right)$, and was $0.263 \mathrm{~mm}(0.207 \mathrm{in})$ in radius. The heat generation in the segregated fuel was calculated from the assumption of pure fuel in a cylinder and was 5.03 times normal fuel. The temperature-dependent conductivity of pure $\mathrm{U}_{3} \mathrm{Si}_{2}$ and the clad was used. The conductivity of the normal fuel $\left(\mathrm{U}_{3} \mathrm{Si}_{2}+\mathrm{Al}\right)$ was varied from $160.0-225.0 \mathrm{~W} / \mathrm{m}^{\circ} \mathrm{C}$ in parametric studies.

Table 2.3 Thermal properties for Kirkpatrick ANSR studies

\begin{tabular}{lcc}
\hline Material & \multicolumn{2}{c}{ Conductivity } \\
\cline { 2 - 3 } & $\mathrm{W} / \mathrm{m}^{\circ} \mathrm{C}$ & $\mathrm{Btu} / \mathrm{h} \cdot \mathrm{in} . .^{\circ} \mathrm{F}$ \\
\hline Fuel inner element & $160.0-225.0$ & $92.1-129$ \\
Segregated fuel @60 & 14.8 & 8.52 \\
Clad @ $60^{\circ} \mathrm{C}$ & 167.3 & 96.3 \\
Oxide & 2.25 & 1.3 \\
\hline
\end{tabular}

The oxide thickness was varied up to $0.03 \mathrm{~mm}$. The convective boundary condition heat transfer coefficient used the Petukhov ${ }^{7}$ correlation with properties for water at ANSR conditions and local surface temperatures. These studies were used to explore some design options for a preliminary ANSR design. 


\section{PREVIOUS ANSR (GILES) ANALYSES (ORNL/TM-12398, 1994)}

Investigations of other design issues pertinent to the fuel plate hot spots were performed over several years by this author ${ }^{8-10}$ with the same basic axisymmetric model philosophy. Many issues were studied during this period. These issues were initially studied by assuming a set of thermal parameters and performing a series of analyses where the meat thickness was varied from the maximum to the minimum design limits. I call this a meat thickness variation study. This section will summarize briefly those studies as a basis to understand the development of a more detailed or a local analysis technique.

\subsection{DESCRIPTION OF MODEL}

The basic model for the ANSR Hot Spot Studies was a two-dimensional axisymmetric model around a postulated nonbond and coincident segregation volume. The nonbond was modeled as a thin adiabatic disk. The diameter of the nonbond was determined from the largest nonbond that would pass the proposed inspection procedures. The thickness of the nonbond disk was assumed to be $0.1 \mathrm{~mm}$. Inspection limits ranging from $1 / 32$ to $1 / 16$ in. diameter were studied. Limited investigations of allowing gas conductivity and radiation across the nonbond were done.

As in the earlier investigations, the segregation volume was modeled as a cylinder the same height as the meat layer (Fig. 3.1). Various inspection limits were analyzed over duration of these studies resulting in segregation volumes of different sizes. The composition of the segregation material was varied from pure solid fuel to a cermet of $50 \%$ fuel, $35 \%$ aluminum and $15 \%$ void. The solid pure material was chosen as in Kirkpatrick's studies as the most concentrated form of the fuel that could exist. This assumption was judged to be too conservative. During tests of the packing of the granulated material, the maximum observed density was $50 \%$. This value was adopted as the upper limit on the maximum density of a segregated material for the later part of the series of studies. The maximum density has a strong influence on the size of the segregation spot. The conductivity of the segregation material was initially determined from interpolation on an experimentally derived curve of conductivity as a function of the percentage of fuel volume. These data were for unirradiated fuel. Irradiation can produce significant material changes. The fuel particles swell due to gaseous fission products and close the voids, initially increasing the conductivity. As the fission density increases, the gaseous fission products will further change the conductivity. Other effects such as recrystallization further modify the 
thermal conductivity. Later studies incorporated results from the DART ${ }^{11}$ code for the homogenized thermal conductivity as a function of the fission density for both the normal and segregated fuel. The DART results for thermal conductivity are determined for a unit volume of the cermet that contains a mixture of fuel and filler particles and some nearly unavoidable voids.

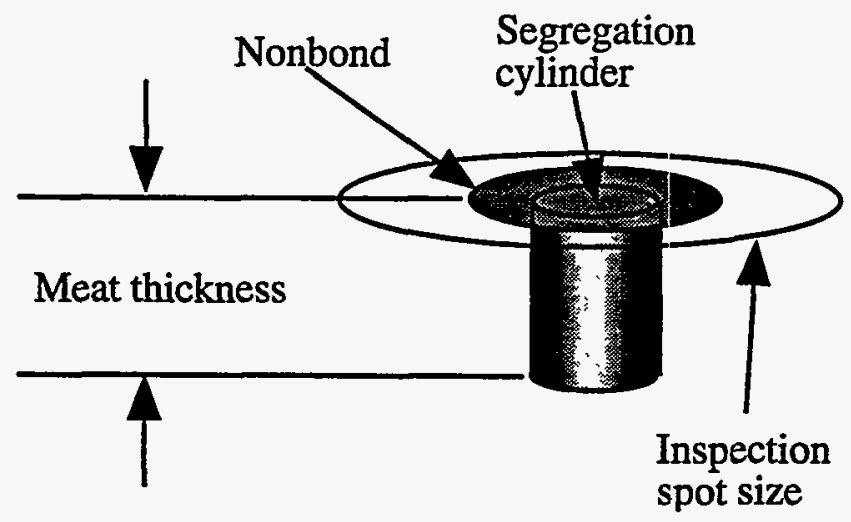

Fig. 3.1. Cylindrical segregation volume model.

\subsubsection{Fuel Thermal Conductivity}

The conductivity of the $\mathrm{U}_{3} \mathrm{Si}_{2}$ fuel particles changes under irradiation due to the generation of gaseous fission products and the formation of compounds not in the original mixture. This complex phenomenon causes the homogenized conductivity of normal fuel to generally decrease with increased fission density (increased burnup). Initial estimates of the homogenized thermal conductivity as a function of the fission density for normal fuel at $93 \%$ enrichment were provided by J. Rest. ${ }^{12}$ Rest also reported an estimate of the more complex behavior of the conductivity of the segregated fuel (also at 93\% enrichment). These initial estimates by the DART code are shown in Fig. 3.2. The gray region on the figure represents the span of fission densities found in the L7 core clesign (a designation for a specified fuel loading) and the vertical line represents the ANSR Design Limit fission density. The thermal conductivity estimate for the segregated material is compared with that of pure unirradiated fuel material in Fig. 3.3. Improved estimates for lower enrichment levels made by an improved DART model with more recent data are reported in Sects. 7 and 8.

It is important to emphasize the significance of the use of homogenized thermal conductivity and its effect on the calculated temperatures. The conductivities used in these calculations will produce temperature estimates that represent the volume averaged temperature within a small volume of the cermet and not the maximum temperature within a single fuel particle. The true maximum temperature of the fuel particles will 
be higher than calculated by these models. This situation is true whenever the homogenized thermal conductivity is higher than that of pure $\mathrm{U}_{3} \mathrm{Si}_{2}$, shown on Fig. 3.3. The importance of these temperatures is discussed more fully in Sect. 7.

The L7 core design and these thermal conductivity estimates were used for the detailed analysis reported in this section.

The thermal conductivity of the normal and segregated fuel used in the thermal analyses is interpolated on these curves by the fission density in the $\mathrm{U}_{3} \mathrm{Si}_{2}$ particles. The fission density, $\mathrm{F}_{\mathrm{d}}$, is calculated from the initial surface density of $235 \mathrm{U}$ and the burnup estimates provided by the VENTURE14 code.

$$
F_{d}=\frac{S_{d} B_{u}}{t m v_{f}}\left(\frac{A_{v}}{M_{w}}\right) \frac{\text { fissions }}{m^{3}}
$$

where

$$
\begin{aligned}
& \mathrm{S}_{\mathrm{d}}=\text { initial surface density, }\left(235 \mathrm{U} \mathrm{g} / \mathrm{mm}^{2}\right) \\
& \mathrm{t}_{\mathrm{m}}=\text { meat thickness, } \mathrm{mm}, \\
& \mathrm{v}_{\mathrm{f}}=\text { volume fraction of fuel }=0.112, \\
& \mathrm{~A}_{\mathrm{v}}=\text { Avogadro's number }=6.023 \times 10^{23}(1 / \mathrm{mol}) \\
& \mathrm{Mw}=\text { molecular weight }=235(\mathrm{~g} / \mathrm{mol}) \\
& \mathrm{B}_{\mathrm{u}}=\text { burnup }=\rho_{\mathrm{uf}} / \rho_{\mathrm{ua}} \\
& \rho_{\mathrm{uf}}=\text { density of fissioned } 235 \mathrm{U}, \\
& \rho_{\mathrm{ua}}=\text { maximum density of } 235 \mathrm{U} \text { atoms that can fission, }
\end{aligned}
$$

A summary of the range of thermal conductivities used in these analyses is included in Table 3.1 .

Table 3.1. Fuel Thermal Conductivities

\begin{tabular}{cc}
\hline Material & (W/m ${ }^{\circ} \mathrm{C}$ ) \\
\hline Normal fuel & (see Fig. 3.2) \\
Beginning of cycle & 170.0 \\
End of cycle at ANSR Design Limit (ANSR peak) & 66.3 \\
Segregated fuel - 50\% $\mathrm{U}_{3} \mathrm{Si}_{2}$ 15\%void 35\%Al & (see Fig. 3.2) \\
Beginning of cycle & 14.5 \\
End of cycle & 24.5 \\
$\mathrm{U}_{3} \mathrm{Si}_{2}$ (unirradiated) & $13.36+2.4 \times 10^{-3} \mathrm{~T}$ \\
\hline
\end{tabular}




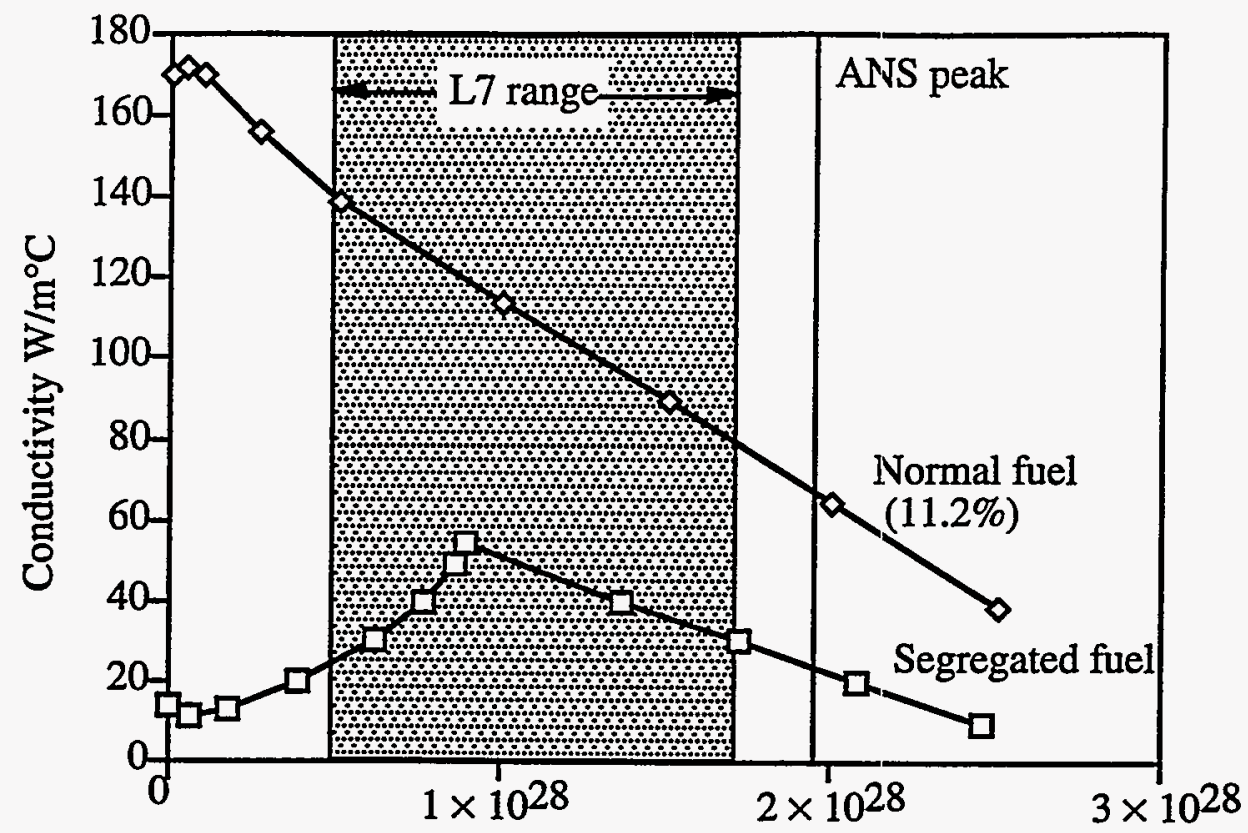

Fission density in $\mathrm{U}_{3} \mathrm{Si}_{2}$ (fission $/ \mathrm{m}^{3}$ )

Fig. 3.2. Comparison of thermal conductivity of normal fuel and segregated fuel as a function of fission density. Both sets of results were produced by the DART code.

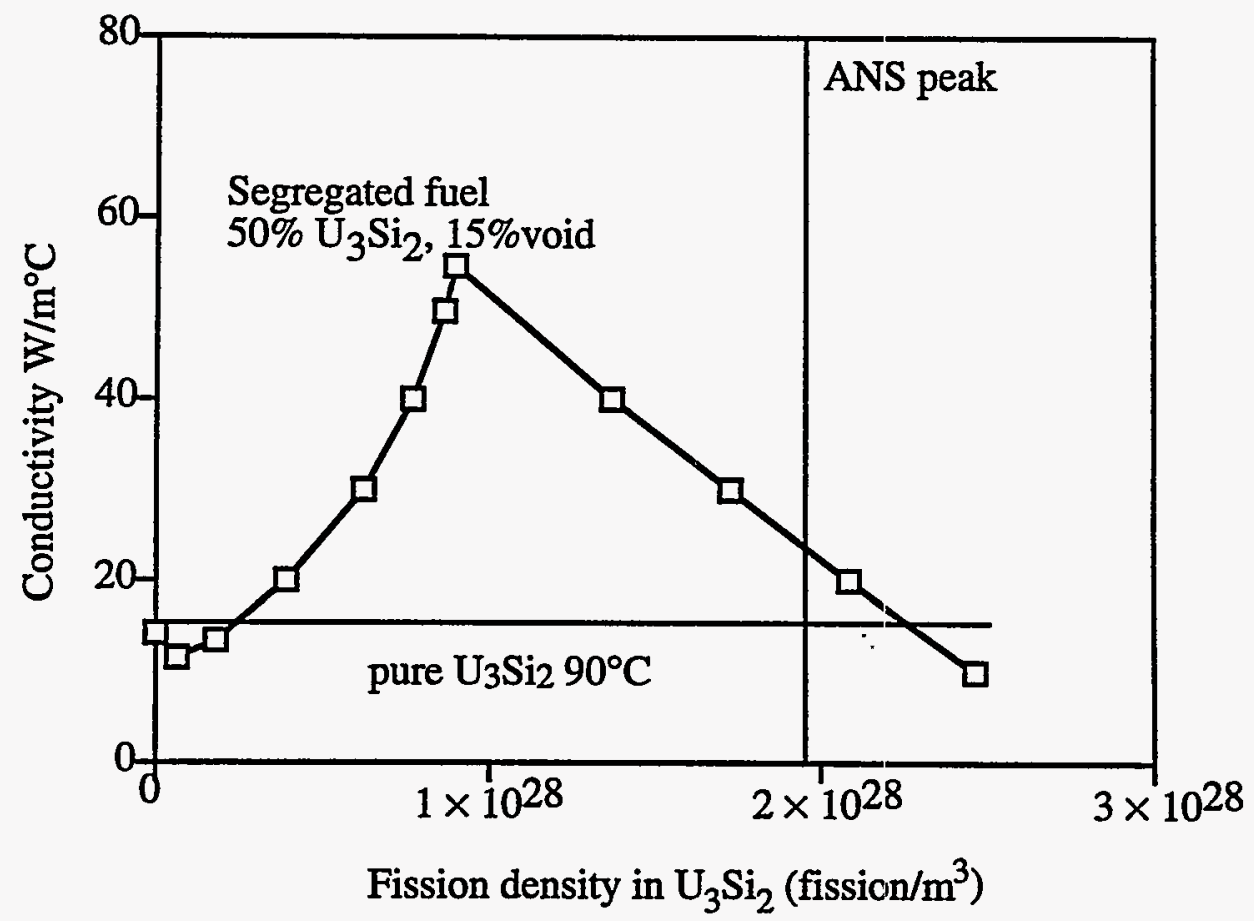

Fig. 3.3. Comparison of thermal conductivity of segregated fuel and unirradiated $\mathrm{U}_{3} \mathrm{Si}_{2}$. 
3.1.2 Fixed Thermal Conductivities

A summary of the thermal conductivities that were not varied during these analyses is included in Table 3.2.

Table 3.2. Fixed Thermal Conductivities

\begin{tabular}{cc}
\hline Material & $\left(\mathrm{W} / \mathrm{m}^{\circ} \mathrm{C}\right)$ \\
\hline Filler & 170.0 \\
Oxide & 2.3 \\
Clad & $15.693+1.8738 \times 10^{-2} \mathrm{~T}-2.5238 \times 10^{-5} \mathrm{~T}^{2}$ \\
\hline
\end{tabular}

\subsubsection{Heat generation}

The heat generation in the fuel meat is a function of the average local heat flux, the maximum design power density, and the meat thickness or

where

$$
q=\frac{M P_{d} H_{a}}{t_{m}}\left(\frac{W}{\mathrm{~mm}^{3}}\right)
$$

$$
\begin{aligned}
& \mathrm{MP}_{\mathrm{d}}=\text { maximum relative power density, } \\
& \mathrm{H}_{\mathrm{a}}=\text { average heat flux }\left(\frac{\mathrm{W}}{\mathrm{mm}^{2}}\right) \\
& \mathrm{T}_{\mathrm{m}}=\text { meat thickness, } \mathrm{mm} .
\end{aligned}
$$

The heat generation in the clad and filler is not known and is assumed to be zero for these calculations. All of the heat generated in the plates is assumed to be concentrated in the fuel meat. These assumptions produce conservative estimates of the peaking factor and maximum temperature by overestimating the heat generation in the fuel meat.

\subsubsection{Coolant Parameters}

The fluid properties for $\mathrm{D}_{2} \mathrm{O}$ flowing in a gap $1.27 \mathrm{~mm}$ high and $70.29 \mathrm{~mm}$ wide were used in the Petukhov correlation to estimate the heat transfer coefficient. The $\mathrm{D}_{2} \mathrm{O}$ properties were obtained from ref. 13 . The Petukhov correlation for heat transfer coefficient as a function of temperature is used with the parameters in Table 3.1. 
Table 3.3. Coolant boundary condition parameters

\begin{tabular}{lll}
\hline Velocity & $2.5 \times 10^{4} \mathrm{~mm} / \mathrm{s}$ & $2.5 \mathrm{~m} / \mathrm{s}$ \\
Hydraulic diameter & $2.494 \mathrm{~mm}$ & $0.002494 \mathrm{~m}$ \\
Gap height & $1.27 \mathrm{~mm}$ & $0.00127 \mathrm{~m}$ \\
Gap span & $70.29 \mathrm{~mm}$ & $0.07029 \mathrm{~m}$ \\
Bulk temperature & $90.0^{\circ} \mathrm{C}$ & $90.0^{\circ} \mathrm{C}$ \\
Reynolds number & $1.8 \times 10^{5}$ & $1.8 \times 10^{5}$ \\
Friction factor & 0.0173 & 0.0173 \\
Prandtl number & 2.4 & 2.4 \\
Dynamic viscosity & $3.44 \times 10^{-10} \mathrm{Ns} / \mathrm{mm}^{2}$ & $3.44 \times 10^{-4} \mathrm{Ns} / \mathrm{m}^{2}$ \\
Fluid conductivity & $0.0634 \mathrm{~W} / \mathrm{mm} \mathrm{K}$ & $6.34 \mathrm{~W} / \mathrm{m} \mathrm{K}$ \\
\hline
\end{tabular}

The Petukhov correlation for Nusselt number, $\mathrm{Nu}_{\mathrm{p}}$, is (ignoring entrance effects):

$$
N_{u_{p}}=\frac{F_{f} \operatorname{Re}_{b} \operatorname{Pr}_{b}\left(\frac{\mu_{b}}{\mu_{w}}\right)^{p .11}}{8\left[\left(1+3.4 F_{f}\right)+\left(11.7+\frac{1.8}{\operatorname{Pr}_{b}^{1 / 3}}\right)\left(\frac{F_{f}}{8}\right)^{/ 2}\left(\operatorname{Pr}_{b}^{2 / 3}-1\right)\right]}
$$

where

$$
\begin{aligned}
& \mathrm{Nu}_{\mathrm{p}}=\frac{\mathrm{h} \mathrm{D}_{\mathrm{h}}}{\mathbf{k}_{\mathrm{b}}} \\
& \mathrm{h}=\text { heat transfer coefficient } \\
& \left.\mathrm{D}_{\mathrm{h}}=\text { hydraulic diameter (for 2-D gaps }=2 \mathrm{H}\right) \\
& \mathrm{H}=\text { height of fluid channel } \\
& \mathrm{k}_{\mathrm{b}}=\text { fluid conductivity at bulk fluid temperature } \\
& \mathrm{Re}_{\mathrm{b}}=\text { Reynolds number at bulk fluid temperature } \\
& \mathrm{Re}_{\mathrm{b}}=\frac{\mathrm{V} \mathrm{D}_{\mathrm{h}}}{\mathrm{u}_{\mathrm{b}}} \\
& \mathrm{V}=\text { fluid velocity } \\
& \mathrm{v}_{\mathrm{b}}=\text { kinematic viscosity at bulk fluid temperature } \\
& \mathrm{Pr}_{\mathrm{b}}=\text { Prandtl number at bulk fluid temperature } \\
& \mu_{\mathrm{b}}=\text { fluid dynamic viscosity at bulk fluid temperature } \\
& \mu_{\mathrm{w}}=\text { fluid dynamic viscosity at wall temperature } \\
& \mathrm{F}_{\mathrm{f}}=\text { Filonenko friction factor }=\left[1.82 \log \left(\mathrm{Re}_{\mathrm{b}}\right)-1.64\right]^{-2}
\end{aligned}
$$

The properties at the specified state were obtained by evaluating the functions defined in ref. 13 using a FORTRAN code. This code also generated a table of the heat transfer coefficient as a function of the temperature from the Nusselt number by the following equation: 


$$
\mathrm{h}=\frac{\mathrm{k}_{\mathrm{b}} N \mathrm{Nu}_{\mathrm{p}}}{\mathrm{D}_{\mathrm{h}}}
$$

\subsubsection{Excess Local Power}

Proposed inspection procedures require that the maximum allowable local excess power be no more than $20 \%$ over a fuel plate area of $2 \mathrm{~mm}$ ( 80 mils) in diameter. Several mechanisms can be postulated to produce this excess. The assumption that would produce the most concentrated uranium volume would be a dense lump of pure $235 \mathrm{U}$ be located at the center of the 2-mm-diameter spot. This scenario, however, appears to be unlikely to occur in the manufacturing process. The next most concentrated segregation spot assumption would be a solid lump of $\mathrm{U}_{3} \mathrm{Si}_{2}$ at the center of the spot. Since the $\mathrm{U}_{3} \mathrm{Si}_{2}$ powder is controlled to have particles smaller than $150 \mu \mathrm{m}$, it is also unlikely that this condition will occur. Since the typical pour density or apparent density of the fuel powders is about $50 \%$, this is taken as the maximum density of an inhomogeneous spot. Rolled fuel containing 50\% ceramic will typically contain $15 \%$ void and $35 \%$ matrix aluminum. ${ }^{1}$ For a segregation spot made up of a cylinder of $50 \%$-density $\mathrm{U}_{3} \mathrm{Si}_{2}$ extending the thickness of the fuel meat layer and surrounded by normal fuel, the segregation cylinder diameter is $0.474 \mathrm{~mm}$. This cylinder segregation model was used for the analyses reported in this section.

An alternative, and perhaps a more conservative assumption, of surrounding the segregated spot with an annular volume containing only filler (no fuel) was not used. No mechanism has been proposed that can produce this condition. (This assumption may not be more conservative because the much higher conductivity of the filler would reduce the effect of the larger $50 \% \mathrm{U}_{3} \mathrm{Si}_{2}$ spot.)

The ratio of heat generation in the segregated fuel to that of normal fuel is assumed to be equal to the ratio of the densities of $235 \mathrm{U}$ in segregated and normal fuel:

where

$$
\mathrm{Q}_{\mathrm{s}}=\left(\frac{\mathrm{p} \rho_{\mathrm{s}}}{\rho_{\mathrm{n}}}\right)
$$

$$
\begin{aligned}
\mathrm{p} & =\text { volume fraction of } \mathrm{U}_{3} \mathrm{Si}_{2}=0.50 \\
\rho_{\mathrm{s}} & =235 \mathrm{U} \text { density in segregated fuel }\left(\frac{235 \mathrm{U}_{\mathrm{mg}}}{\mathrm{mm}^{3}}\right) \\
& =\mathrm{Mu} \mathrm{En}=10.472 \mathrm{mg} / \mathrm{mm}^{3} \\
\rho_{\mathrm{n}} & =235 \mathrm{U} \text { density in normal fuel }\left(\frac{235 \mathrm{U}_{\mathrm{mg}}}{\mathrm{mm}^{3}}\right) \\
& =\text { En } \rho_{\mathrm{tn}}=1.1184
\end{aligned}
$$




$$
\begin{aligned}
\mathrm{Mu} & =\text { mass of uranium (all isotopes) in } \mathrm{U}_{3} \mathrm{Si}_{2}=\mathrm{r}_{\mathrm{ts}} \rho_{1} \\
\mathrm{r}_{\mathrm{ts}} & =0.921 \frac{\mathrm{TU}}{\mathrm{U}_{3} \mathrm{Si}_{2}} \\
\rho_{1} & =\text { density of pure } \mathrm{U}_{3} \mathrm{Si}_{2}=12.2 \mathrm{mg} / \mathrm{mm}^{3}\left(12.2 \mathrm{~g} / \mathrm{cm}^{3}\right) \\
\mathrm{En} & =\text { enrichment }=0.932 \frac{23 \mathrm{U}}{\mathrm{TU}} \\
\rho_{\mathrm{tn}} & =1.2 \frac{\mathrm{TUmg}}{\mathrm{mm}^{3}}
\end{aligned}
$$

For $50 \% \mathrm{U}_{3} \mathrm{Si}_{2}, \mathrm{Q}_{\mathrm{s}}=4.681$ relation

The diameter of the segregation spot $\left(d_{s}\right)$ is calculated from the

$$
1.0 \mathrm{~A}+\left(\mathrm{Q}_{\mathrm{s}}-1.0\right) \frac{\mathrm{d}_{\mathrm{s}}^{2} \pi}{4}=1.2 \mathrm{~A}
$$

where

$\mathrm{d}_{\mathrm{s}}=$ diameter of the segregation spot,

$\mathrm{d}_{\mathrm{i}}=$ diameter of the inspection spot $=2.03 \mathrm{~mm}$,

$\mathrm{A}=$ area of the inspection spot $=\frac{\mathrm{pd}_{\mathrm{i}}^{2}}{4}=3.24 \times 10^{-6} \mathrm{~m}^{2}$,

$d_{s}=\sqrt{\frac{0.8 A}{3.681 \mathrm{p}}}=0.473 \mathrm{~mm}(473 \mu \mathrm{m})$ for $50 \% \mathrm{U}_{3} \mathrm{Si}_{2}$.

The excess local heat generation is also a function of the average local power or

where

$$
\mathrm{q}_{\mathrm{s}}=\frac{\mathrm{Q}_{\mathrm{s}} \mathrm{p}_{\mathrm{d}} \mathrm{H}_{\mathrm{a}}}{\mathrm{t}_{\mathrm{m}}}
$$

$$
\begin{aligned}
& \mathrm{p}_{\mathrm{d}}=\text { local relative power density } \\
& \mathrm{H}_{\mathrm{a}}=\text { average heat flux, } \\
& \mathrm{t}_{\mathrm{m}}=\text { meat thickness }
\end{aligned}
$$

The shape of the segregation volume is not known. Shapes other than cylinders are possible. One alternative shape investigated was a disk of segregated material. The size of the disk was determined from the possibility of the segregation volume in the fuel meat being a cause of the metallurgical bond failure. Thus, the segregation volume and nonbond would be coincident. If the segregation spot causes the nonbond then it is logical to assume that both would have the same diameter. If the nonbond were the 
same diameter as the segregation cylinder model, then this nonbond size would not be detected. One cannot ensure that the nonbond size will be smaller than the maximum allowable. Therefore, the nonbond must be assumed to be the maximum allowable size and the segregation spot must assumed to be the same diameter. Because maximum allowable segregation volume is less than that of a cylinder with the nonbond diameter $(1 \mathrm{~mm})$, the simplest segregation region to meet these conditions is a disk adjacent to the nonbond. The nonbond/segregation spot is conservatively assumed to be located at the interface between the fuel meat and filler. Figure 3.4 compares the geometry of a cylinder segregation model with a disk segregation model for the thickest fuel meat zone.

The local power excess can also be produced by a uniform distribution of higher power fuel material in the inspection area. Although this assumption is probably the least conservative possible, it was also investigated to quantify the benefits of better control of the manufacturing process.

\subsection{PHENOMENA NOT INCLUDED IN THESE INVESTIGATIONS}

\subsubsection{Fuel Swelling}

The fuel swells under irradiation due to the production of gaseous fission products. Although individual fuel particles increase $50 \%$ in size, this increase produces a maximum of only $6 \%(0.07 \mathrm{~mm})$ growth in total plate thickness during the fuel cycle. This thickness change has not been included in these types of studies since the increase in fuel volume will produce a reduction in local heat generation and thus a slight reduction in peaking factor and maximum temperature at the end of the cycle. Fuel swelling may also apply enough force to decrease the size or close the nonbond completely. Maintaining the initial geometry is a conservative assumption.

\subsubsection{Plate Bowing}

Plate bowing can affect the coolant channel width, bulk temperature, heat transfer coefficient and local plate geometry. The curvature of the fuel plate is ignored in these studies since the hot spot model radius is small and the plate surfaces are essentially flat over this region. The small changes in the local curvature that can be produced by bowing should not affect these analyses. Local changes in the thicknesses of the plate components are not expected due to the small amount of bowing allowed.

As with the oxide layer thickness, the effects on the heat transfer into the coolant caused by bowing are incorporated into the LAT by the use of TASHA coolant temperatures and by modifying heat transfer coefficient for a minimum thickness channel. 


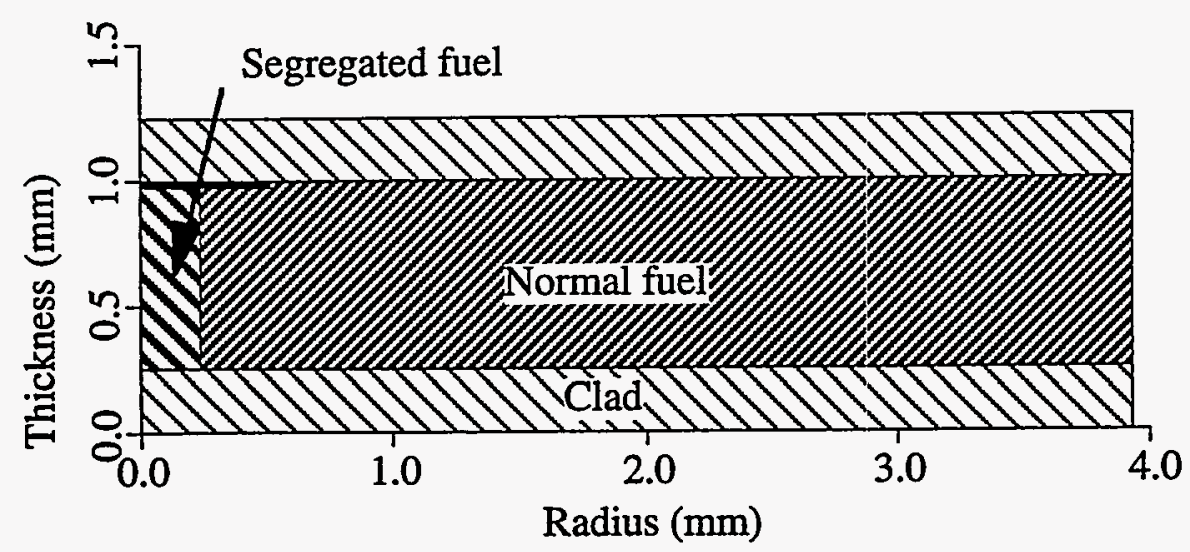

(a)

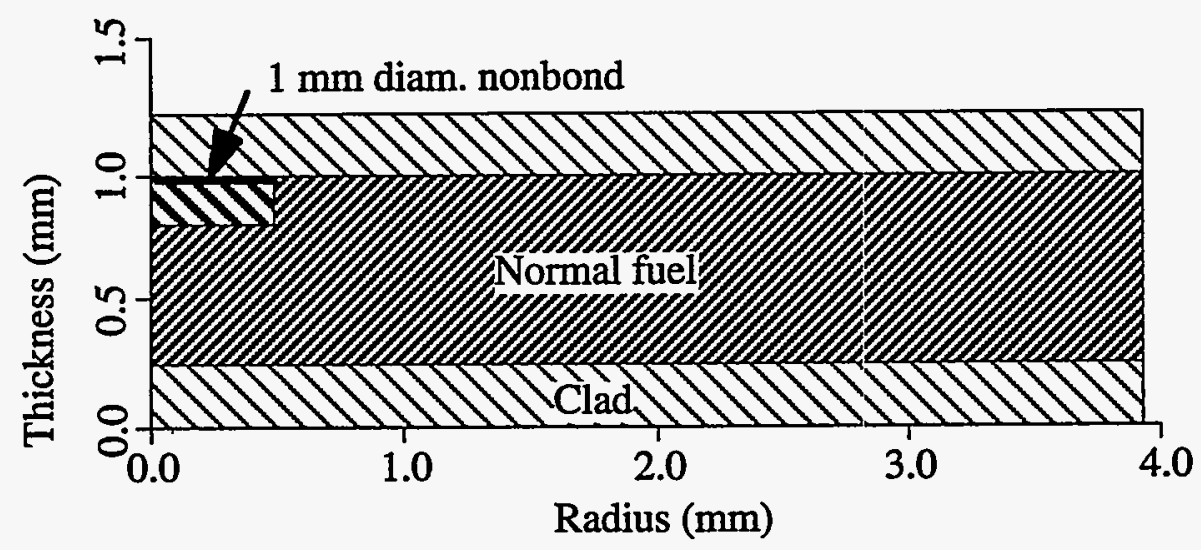

(b)

Fig. 3.4. Segregation volume models for maximum meat layer thickness, (a) cylinder and (b) disk.

\subsection{MEAT THICKNESS VARIATION ANALYSES}

Previous HFIR and ANSR hot spot studies used conservative estimates on the thermal parameters in the idealized nonbond and segregation volume model to determine maximum possible peaking factors and fuel temperatures. Usually the conservative limits on all parameters except the meat thickness were set and the meat thickness was varied between the maximum and minimum possible to produce an estimate of the maximum possible peaking factor and fuel temperatures that can occur anywhere in a core. These analyses are conservative estimates on the bounding conditions because it is highly unlikely that all of the conservative limits will occur at the same time and place in the core. The limited number of analyses needed to bound the conditions is highly desirable when design studies or quick-look calculations are required. However, the overly 
conservative nature of this approach is not adequate for ANSR safety calculations. This is shown in Sects. 5 through 8.

The thermal parameters that are usually fixed in these analyses include the fuel relative power density and burnup, the coolant bulk temperature and flow rate, and the oxide layer thickness. The fuel relative power density, together with the meat thickness, determines the heat generation in the idealized model of the coincident nonbond and segregation volume. The fuel burnup determines the normal and segregated fuel conductivities, and the coolant conditions determine the forced convection heat transfer coefficient.

These types of analyses were used to estimate the maximum peaking factor and fuel temperatures. The maximum fuel temperatures limit $\left(400^{\circ} \mathrm{C}\right)$ is set by fuel swelling limits. The maximum peaking factor estimate produced from these analyses is used in subsequent thermal hydraulic calculations using the TASHA code. The peaking factor affects many parts of the TASHA analysis, but basically the lower the peaking factor the easier it is to achieve the ANSR goals and the larger are the operating margins.

Conservative estimates for the peaking factor are determined by using the minimum oxide layer thickness, maximum coolant flow rate and minimum coolant temperature. However, conservative estimates for fuel temperatures require the use of maximum oxide thickness and coolant temperatures and minimum coolant flow rates. Minimum fuel conductivities will also increase the maximum temperatures. The complex nature of the heat transfer problem and the behavior thermal conductivity as a function of fission density make difficult a definitive statement of which combination of thermal conductivities is strictly conservative. Although some analyses were performed with thermal values that were not strictly conservative, these analyses were useful in making general observations about the effects of various design options.

One such study was used to determine the effect of the shape of the segregation volume. Most of the previous analyses were performed with a circular cylinder composed of highly concentrated segregation material. One of the other shapes investigated was a disk of segregated material. The disk shape was prompted by the suggestion that the segregation volume could cause a nonbond by inhibiting the metallurgical bonding because of the difference in materials at the fuel filler interface. This idea led to considering a disk-shaped segregation volume that was the same diameter as the minimum detectable nonbond. The inspection procedure for excess fuel loading and the assumption of the maximum possible concentration of the segregated material determines the thickness of the disk. The disk, being larger in radius than the cylinder, does not need to extend completely through the meat layer in order to provide the same excess fuel loading as a cylinder that extends completely though the fuel meat. 
The results using a set of representative thermal parameters (but not strictly conservative) are reported in Figs. 3.5 and 3.6. The results show that the cylinder model of the segregation volume produces a more conservative peaking factor. It is also more conservative in predicting maximum fuel temperatures except for the largest fuel meat thickness.

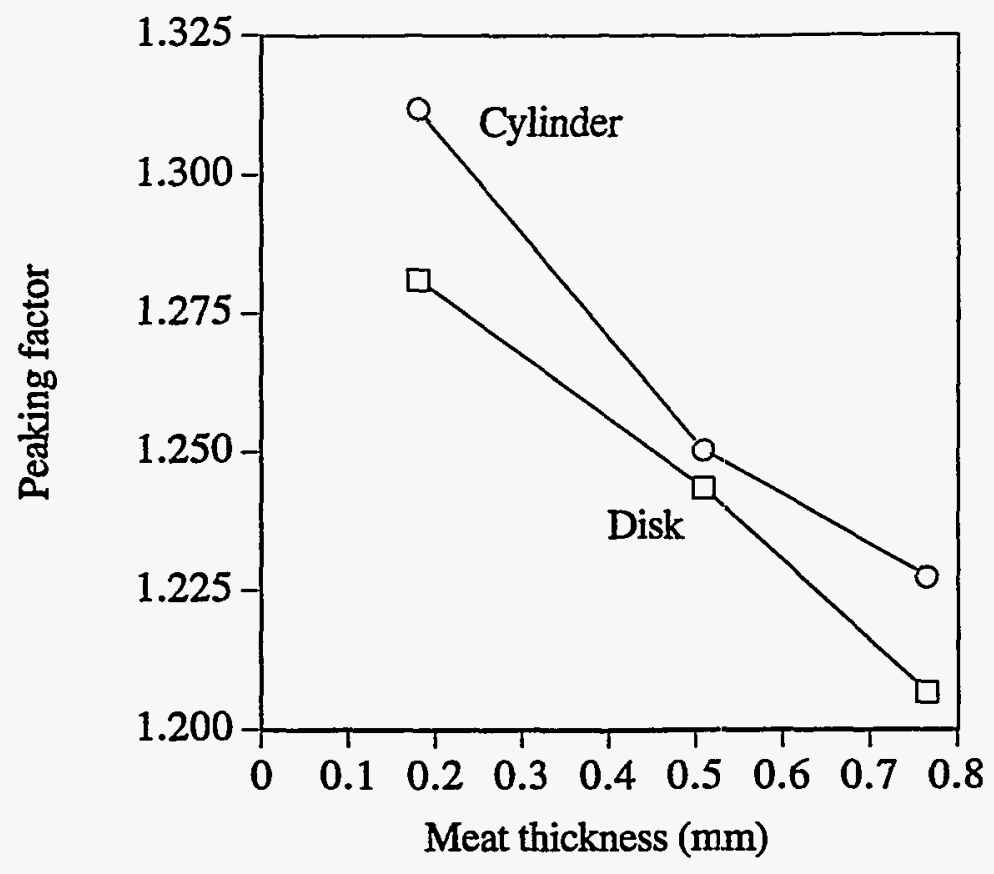

Fig. 3.5. Comparison of peaking factor between models using a cylinder or disk segregation volume.

It is not possible to be sure that the segregation volume would not concentrate into a cylinder; therefore, to be conservative, this shape was chosen for analysis. There is a small region (near maximum meat thicknesses) in which the cylinder model is not the most conservative with respect to the maximum temperatures. Most core designs limit the maximum meat thickness to less than the maximum considered here. In general due to manufacturing tolerances, the maximum meat thickness is $0.7366 \mathrm{~mm}$ rather than the $0.762 \mathrm{~mm}$ used in these analyses. This reduces the region of less than strict conservatism for the cylinder model to a very small area within the fuel plate and a very small temperature difference.

These analyses comparing the cylinder and disk segregation volumes are indicative of many such analyses using typical or representative thermal parameters. Such types of analyses are invaluable tools in making design option comparisons. However, safety analysis after the design has been generally fixed require more conservative choices for thermal parameters. The conservative estimates for the thermal parameters are therefore a deciding factor in the performance of the reactor. 


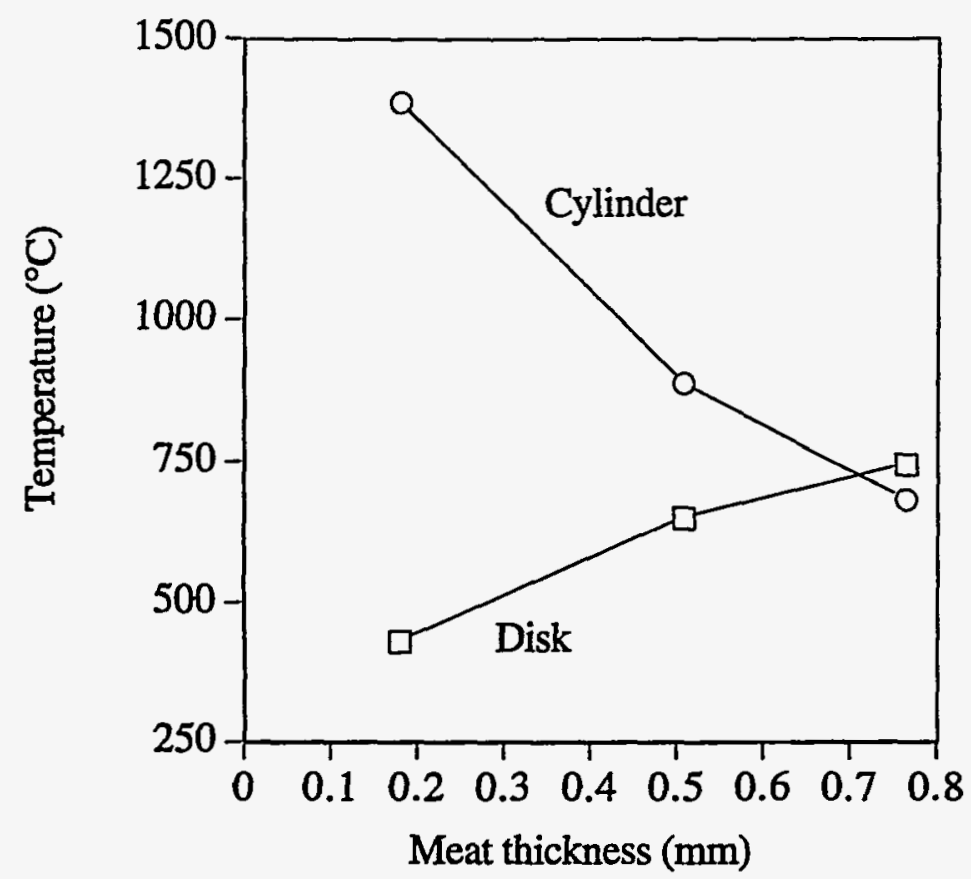

Fig. 3.6. Comparison of maximum temperatures between models using a cylinder or disk segregation volume.

\subsection{ANALYSES USING DESIGN LIMIT AS CONSERVATIVE ESTIMATE}

These investigations involved a series of meat thickness variation thermal analyses using the 2-D axisymmetric hot spot model discussed above. These HEATING7.2 calculations used the ANSR thermal design limits (Table 3.4) as conservative estimates. The design limits were set by various considerations and are used as targets that are not to be exceeded by any specific design. Operation within the design limits is possible and typical. These limits are bounding conditions that simplify the analysis procedure and allow investigation of the variation of other thermal parameters.

Variations in nonbond size, segregation factor, fuel meat location, coolant conditions, thermal conductivities, segregation models, and oxide thickness have been investigated in these studies. The following figures report a few selected results. Figure 3.7 reports a summary of the hot side peaking factor as a function of fuel meat thickness for models including a 1-mm-diameter nonbond only, a segregation cylinder only, and a nonbond with a segregation cylinder. All these results were for clean fuel plates (no oxide layer). The fuel meat layer was centered in the fuel volume. Figure 3.8 presents the maximum temperature results from these analyses. The 
conclusions of these studies are that the maximum peaking factors are on the order of 1.3 but that the maximum temperatures could exceed the $400^{\circ} \mathrm{C}$ limit.

Table 3.4

ANSR Thermal design limits pertinent to hot spot investigations

\begin{tabular}{lc}
\hline Relative power density & 2.31 \\
Maximum burnup $\left(\mathrm{B}_{\mathrm{u}}\right) \mathrm{EOC} *$ & 0.925 \\
Fuel conductivity $\left(\max . \mathrm{B}_{\mathrm{u}}\right)^{*}$ & $66.3 \mathrm{~W} / \mathrm{m}^{\circ} \mathrm{C}$ \\
Segregated fuel conductivity $\left(\max . \mathrm{B}_{\mathrm{u}}\right)^{*}$ & $24.5 \mathrm{~W} / \mathrm{m}^{\circ} \mathrm{C}$ \\
Minimum meat thickness & $0.1778 \mathrm{~mm}$ \\
Maximum meat thickness & $0.762 \mathrm{~mm}$ \\
Maximum oxide thickness EOC & $30.0 \mu \mathrm{m}$ \\
Minimum oxide thickness & $0.0 \mu \mathrm{m}$ \\
\hline
\end{tabular}

*est. from ref. 14

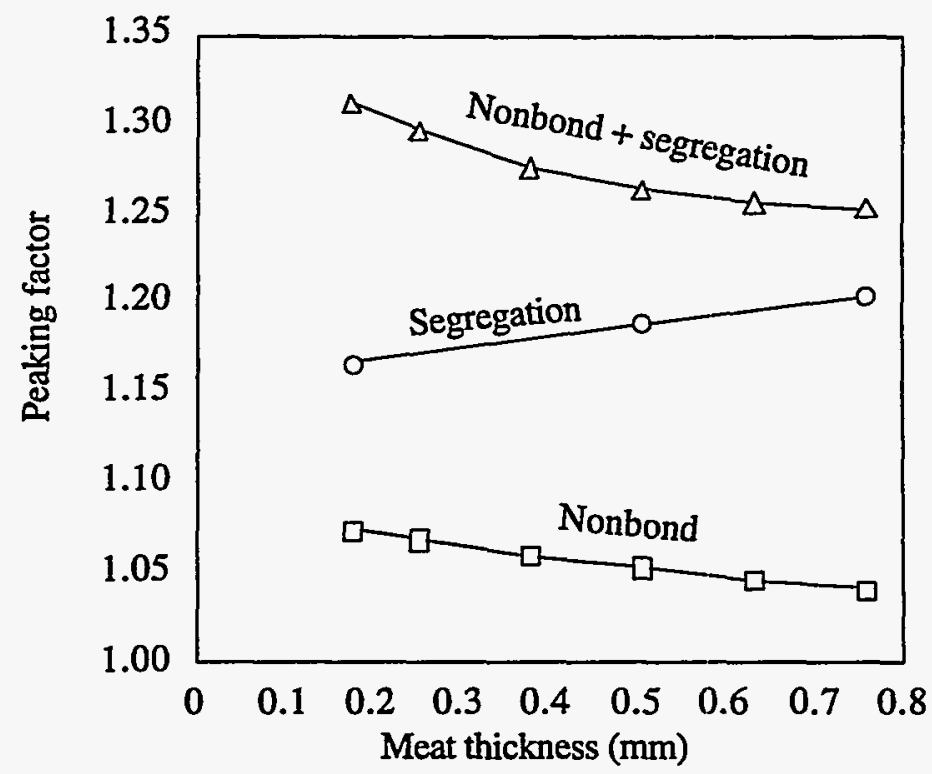

Fig. 3.7. Peaking factor as a function of meat thickness for combinations of fuel defects.

These and the earlier studies were designed to determine the maximum peaking factor and not necessarily the maximum temperature. The maximum forced convection heat transfer coefficient was chosen for these analyses to produce the maximum peaking factor estimates. The minimum heat transfer coefficient should be used for a more conservative maximum temperature estimate. Also, the coolant temperature used in these analyses was the average temperature at the exit, but not necessarily the maximum 
coolant temperature in the core. A more conservative assumption would be to use the maximum coolant temperature. Although the temperatures from these studies are not strictly conservative, trends in results between models should be reflected accurately.

The existence of a 30-micron oxide layer was also studied, and these results are reported in Figs. 3.9 and 3.10. The maximum possible oxide layer reduced the peaking factors, but it elevated the maximum temperatures. These results heightened the concern about the maximum fuel temperatures.

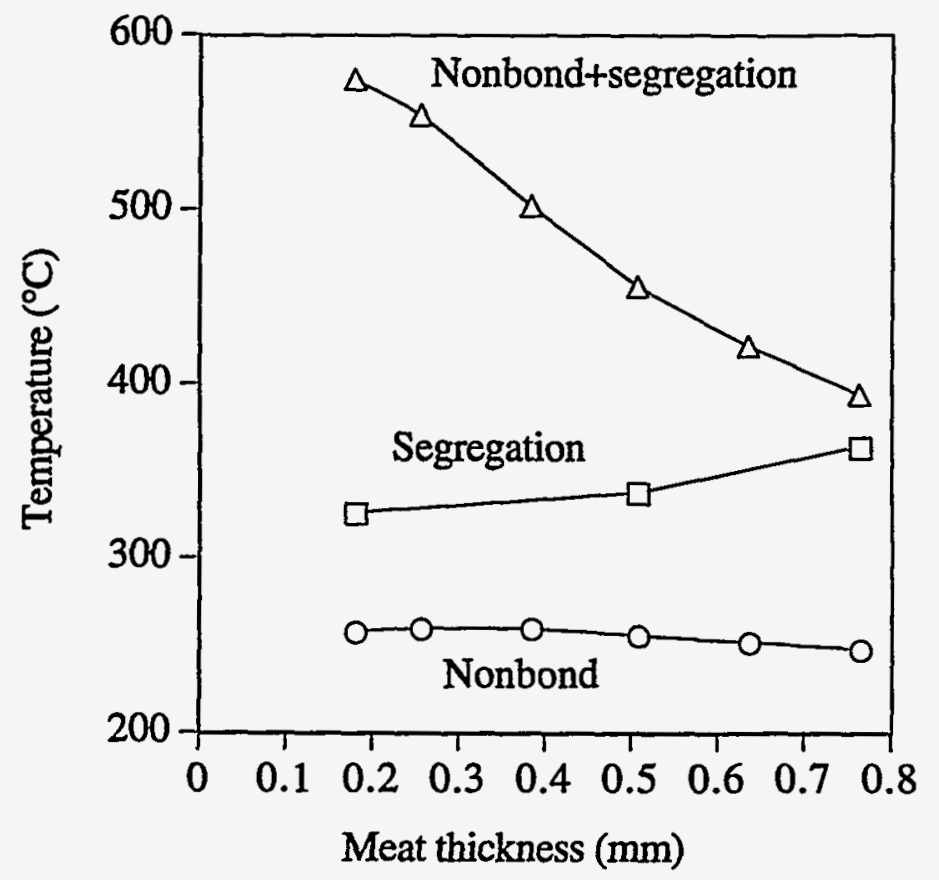

Fig. 3.8. Maximum fuel temperature as a function of meat thickness for combinations of fuel defects.

Meat thickness variation studies were also used to explore various design options. The decision to require centered fuel meat in the fuel volume was prompted by thermal results showing the maximum peaking factor for the fuel meat in various positions. Figure 3.11 presents a comparison of the peaking factors for an ANSR fuel meat placed adjacent to the clad (as in HFIR) with a centered fuel meat. The high peaking factor of 1.85 produced by the $100 \%$ offset fuel placed unacceptable limits on ANSR operating conditions that reduced the likelihood of meeting the design requirements. Even a small variation of $0.0254 \mathrm{~mm}(1 \mathrm{mil})$ from the centered placement would increase the peaking factors, as shown in Fig. 3.11. Clearly manufacturing tolerances would have to be controlled as much as possible and an uncertainty due to meat layer placement would have to be considered 
in the operating conditions. The maximum temperatures were relatively unaffected by the fuel layer placement, as shown in Fig. 3.12.

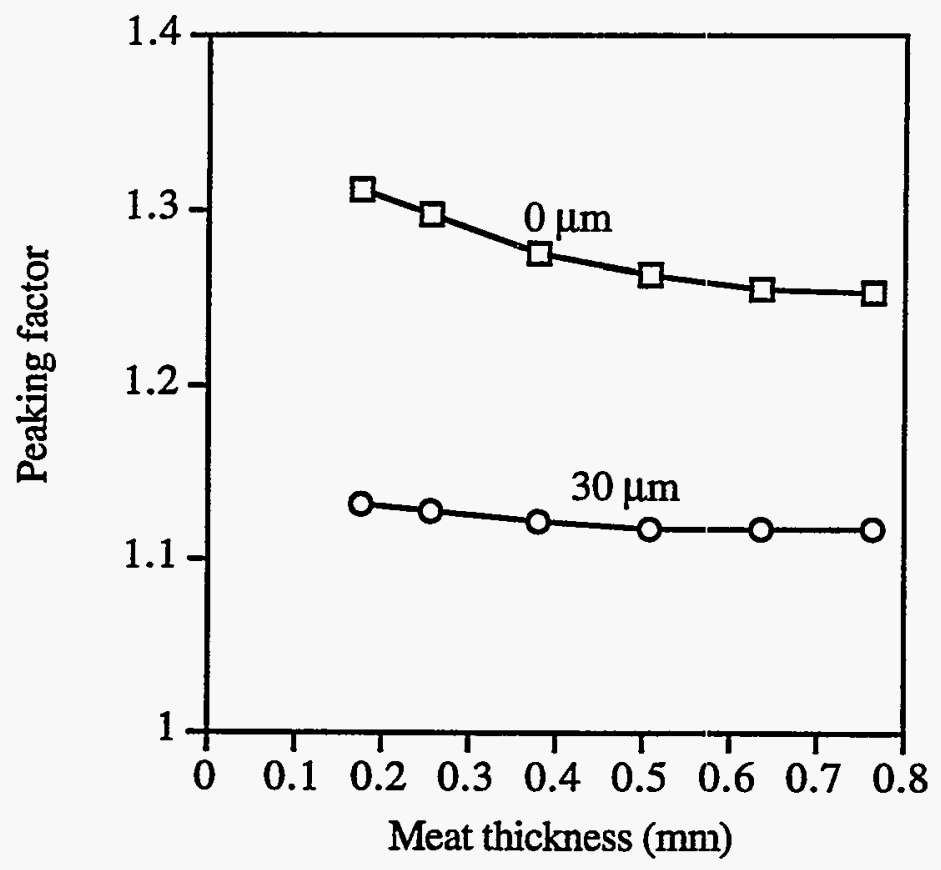

Fig. 3.9. Peaking factor as a function of meat thickness for design limit values with maximum and minimum oxide layer thickness.

\subsection{ANALYSES USING L7 CORE DESIGN WORST-CASE VALUES}

Due to conflicting constraints in the design process, a particular core design may not have all parameters at the ANSR design limits. This situation can allow a slight but significant modification in the conservative estimates for the thermal parameters used in the meat thickness variation analyses. A meat thickness variation study was performed using the thermal parameters found in the L7 core design. The L7 core is a two fuel element core with the inner and outer elements displaced axially from each other. This general arrangement is similar to the core design shown in Fig. 1.1. The L7 designator refers to the meat thickness distribution within the general ANSR fuel plate design. These distributions are shown in detail in Appendix D.

The maximum and minimum meat thicknesses used in the L7 core design are not equal to the design limits. Also, the maximum power density occurs early in the fuel cycle when the fuel conductivities are generally highest. The maximum power at the end of the cycle, when the fuel conductivities are lowest, is much lower than the design limits. The meat thickness variation studies were performed spanning a range from the 
maximum to the minimum values found in the L7 core design for end-of-cycle (EOC) thermal parameters. Table 3.5 presents a summary of the values that were used in the analyses. Figure 3.13 presents a comparison of the hot side peaking factor for L7 design values and design limit. A significant reduction in peaking factor was achieved. Figure 3.14 presents a comparison of the approximate maximum temperature for these studies. Maximum temperature for design values with no oxide is about the same as those for the actual maximum values found in the L7 design.

This comparison demonstrates that a significant reduction in the peaking factor is possible. The estimated maximum temperatures do show a significant reduction but still exceed the thermal limit.

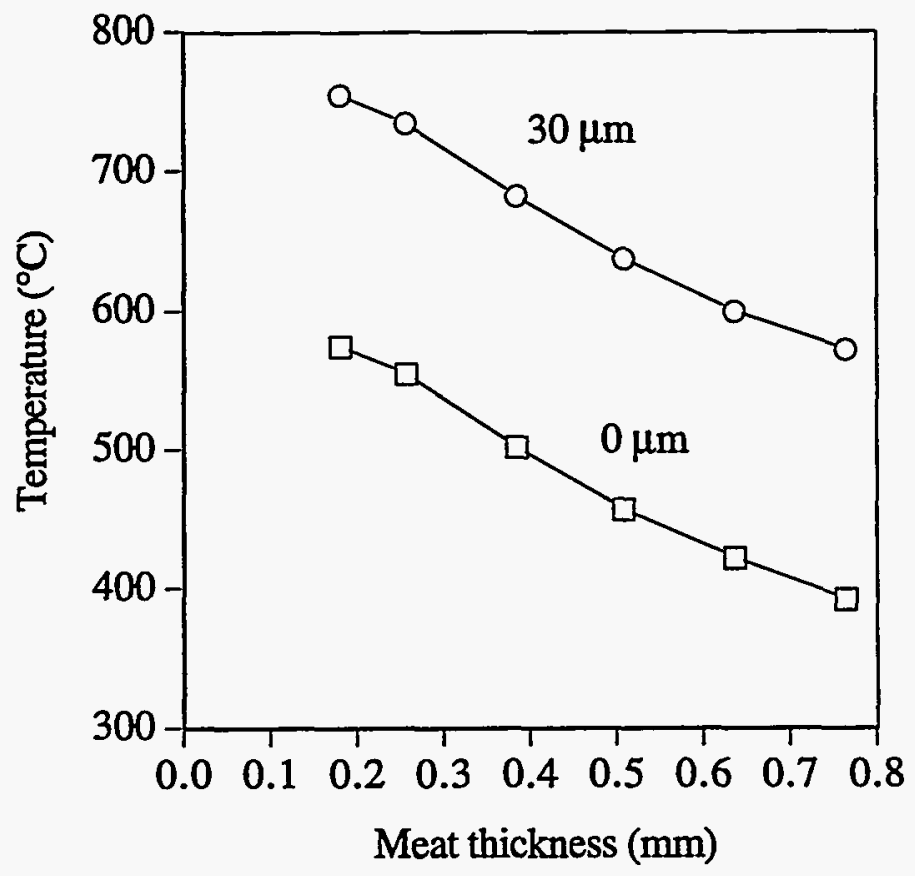

Fig. 3.10. Maximum temperature as a function of meat thickness for design limits values with maximum and minimum oxide layer thickness. 
Table 3.5. Summary of $\mathrm{L} 7$ core design values at EOC

Maximum relative power density

Maximum fuel burnup

Fuel conductivity at maximum burnup

Segregated fuel at maximum burnup

Minimum meat thickness

Maximum meat thickness

Minimum oxide thickness

Maximum oxide thickness
1.7803

0.799

$79.5 \mathrm{~W} / \mathrm{m}^{\circ} \mathrm{C}$

$30.9 \mathrm{~W} / \mathrm{m}^{\circ} \mathrm{C}$

$0.2214 \mathrm{~mm}$

$0.7366 \mathrm{~mm}$

$1.431 \mu \mathrm{m}$

$22.334 \mu \mathrm{m}$

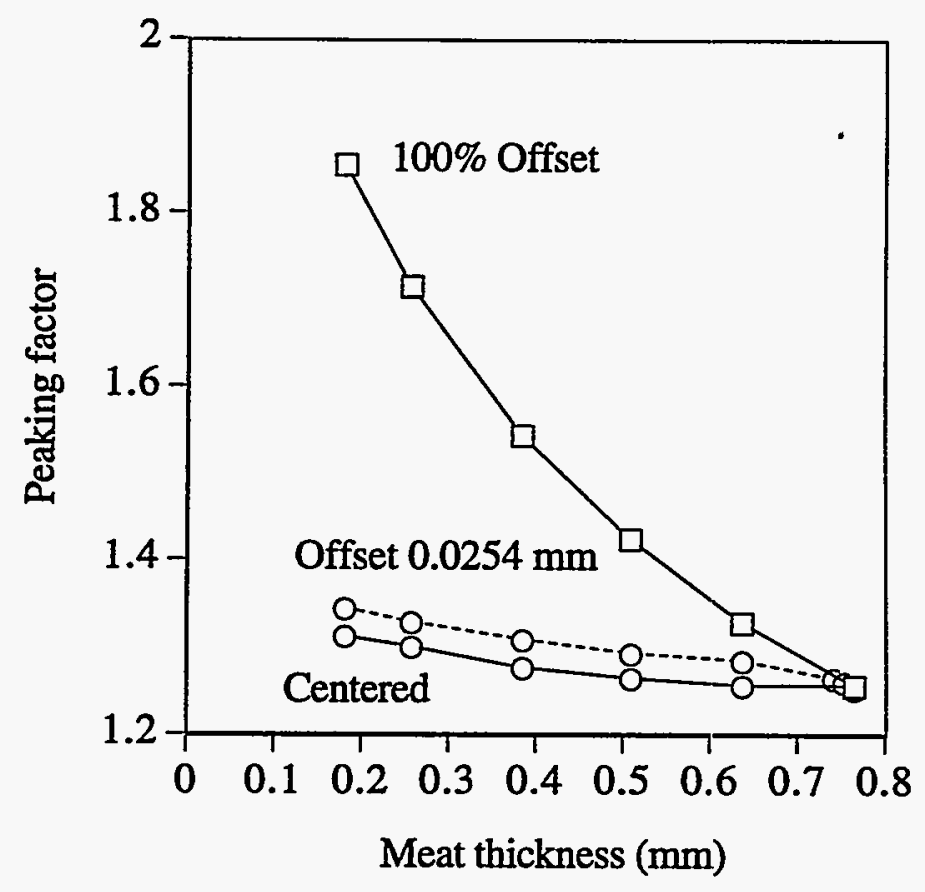

Fig. 3.11. Comparison of the peaking factors for models with fuel meat layers that were centered or offset. Results used design limit values. 


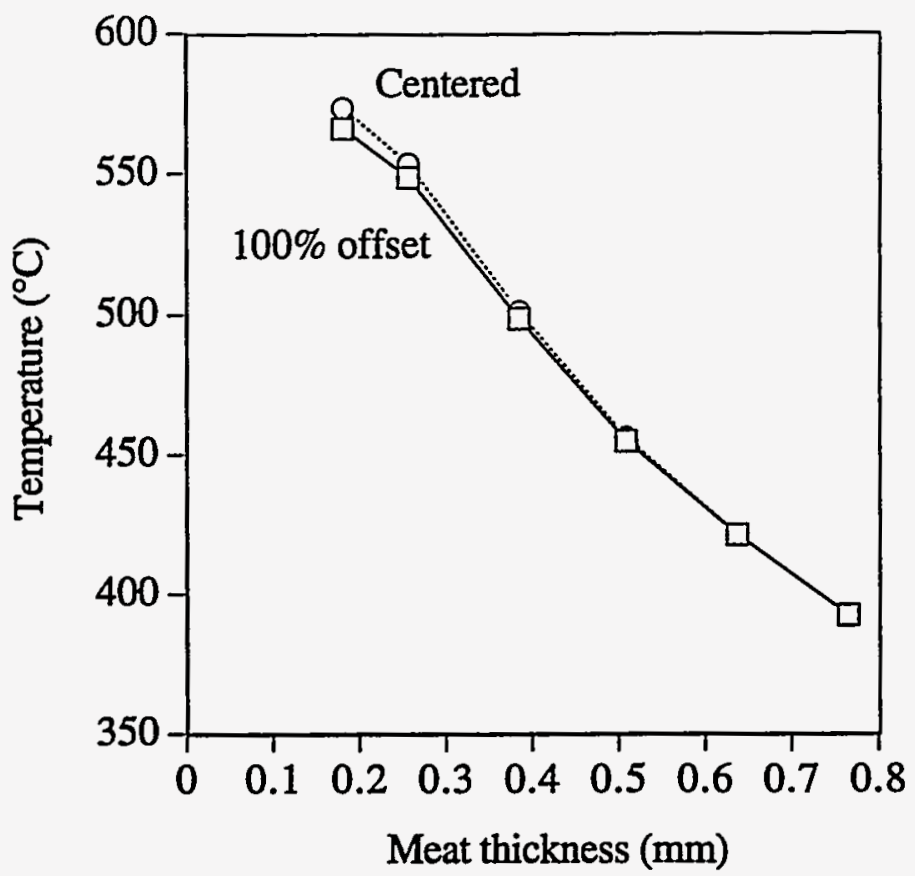

Fig. 3.12. Comparison of the maximum temperatures for models with fuel meat layers that were centered or offset. Results used design limit values.

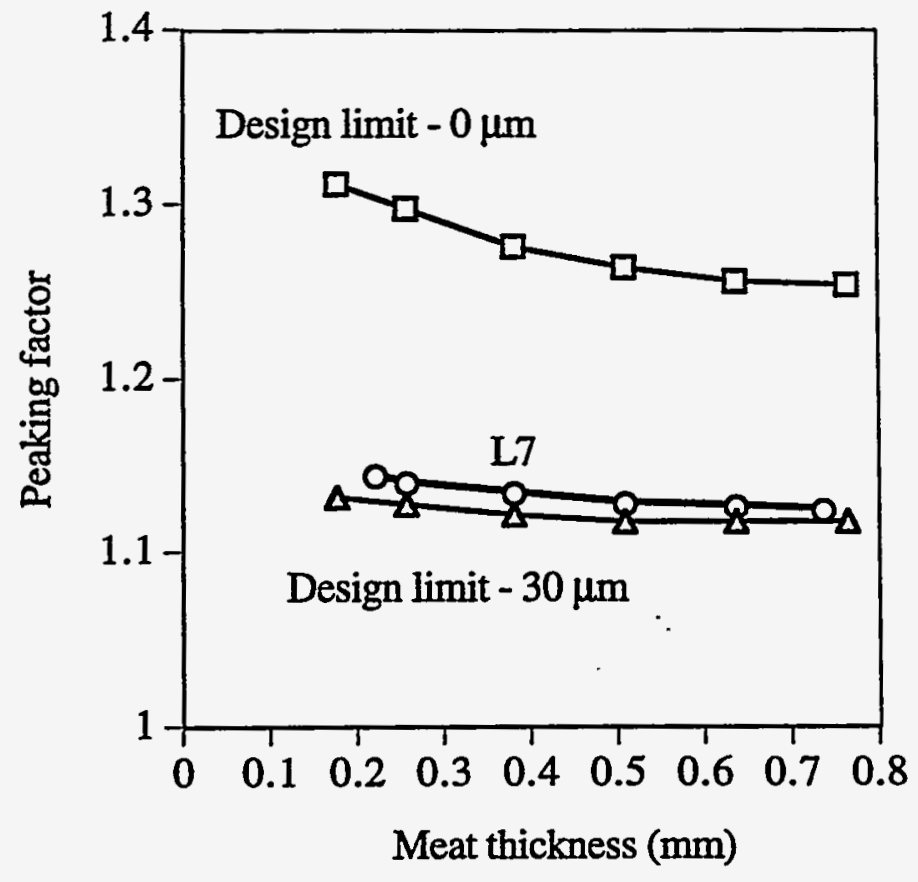

Fig. 3.13. Maximum peaking factors for design values is more conservative than the actual maximum values found in the L7 design. 


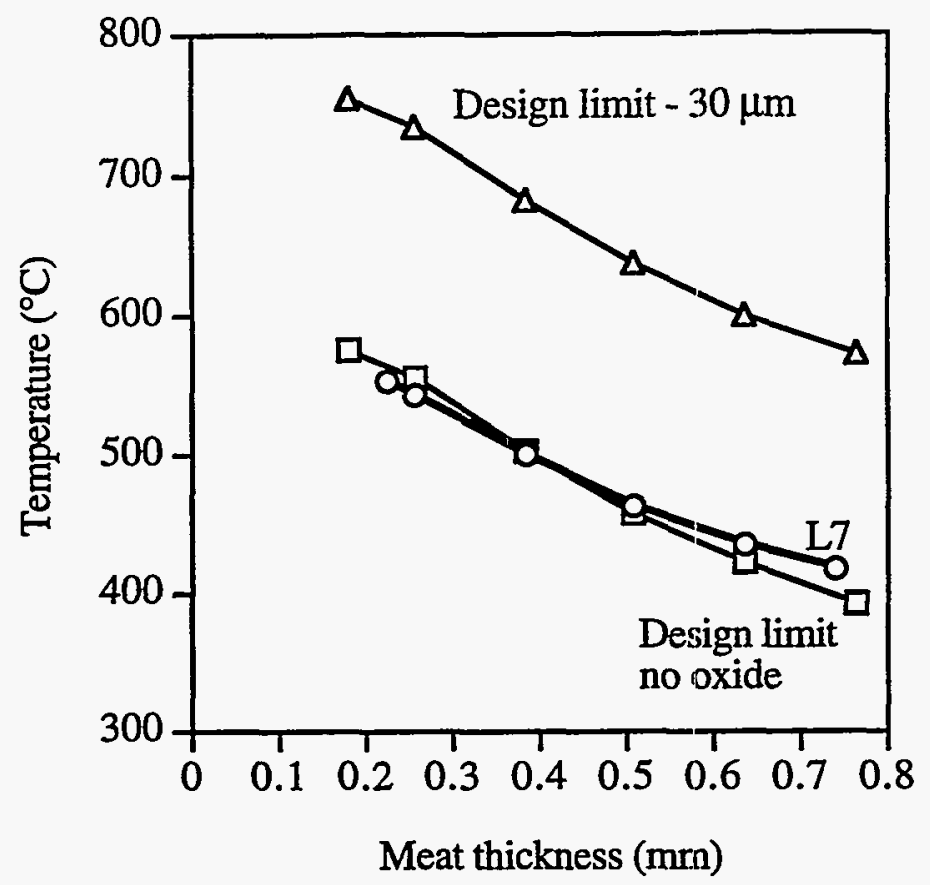

Fig. 3.14. Maximum temperatures for design limits at maximum and minimum oxide thickness compared with worst-case values from the L7 core design.

The investigation of the effect of offsetting the meat layer was also performed for the L7 values, and the results are reported in Fig. 3.15. The amount of offset for each curve is labeled in Fig. 3.15, and models describing the location of the meat layer are shown in Fig. 3.16. The centered meat model is labeled $0 \%$ offset. The curve labeled "0.0254 mm" is from a case with a constant offset of $0.0254 \mathrm{~mm}$ ( $1 \mathrm{mil})$. The offset fuel layer makes a significant difference in the peaking factor but no difference in the maximum temperature. Even with the less strict thermal parameters from the L7 core design, the peaking factors become excessive for a meat layer that is not close to the center.

The conclusion from these studies is that the meat must be close to the center to reduce the peaking factors to acceptable levels. This conclusion applies even in the less-strict analyses using the L7 core values. Also, the maximum fuel temperatures still exceed the design limit of $400^{\circ} \mathrm{C}$. In order to demonstrate that the ANSR design will function as intended, alternative analysis techniques will be needed to reduce the llevel of conservatism while establishing the overall safety of the reactor operation. The technique developed to accomplish these goals is described in the next section. 


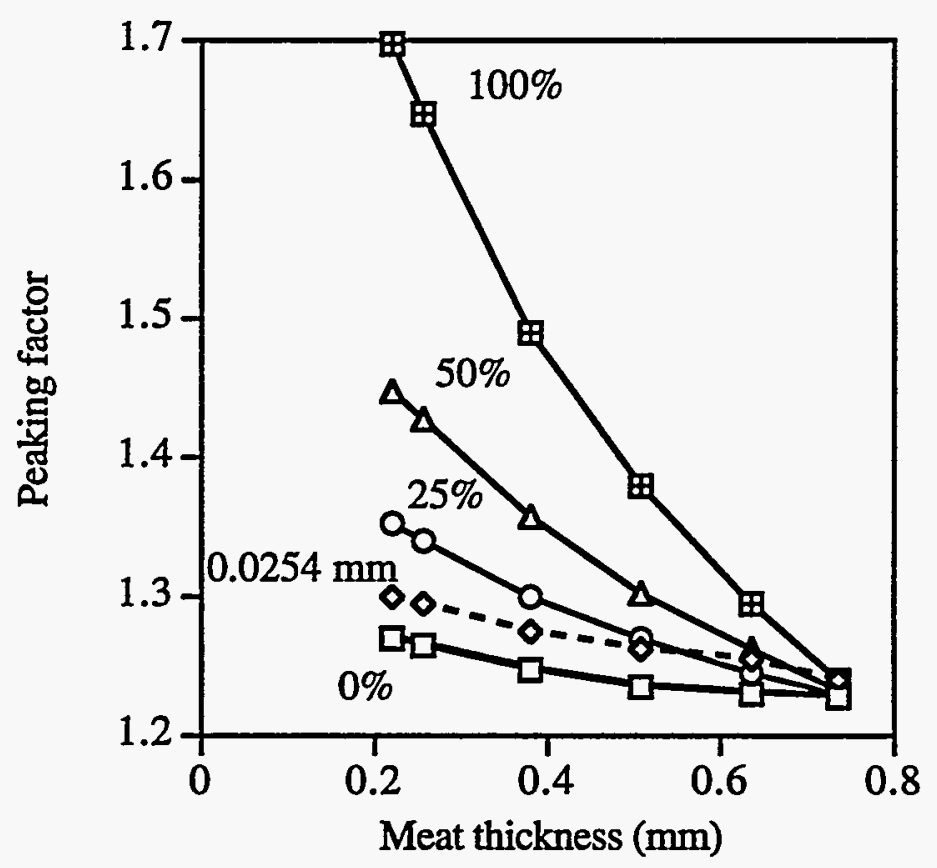

Fig. 3.15. Peaking factors comparison for offset models. L7 worst-case parameters. 

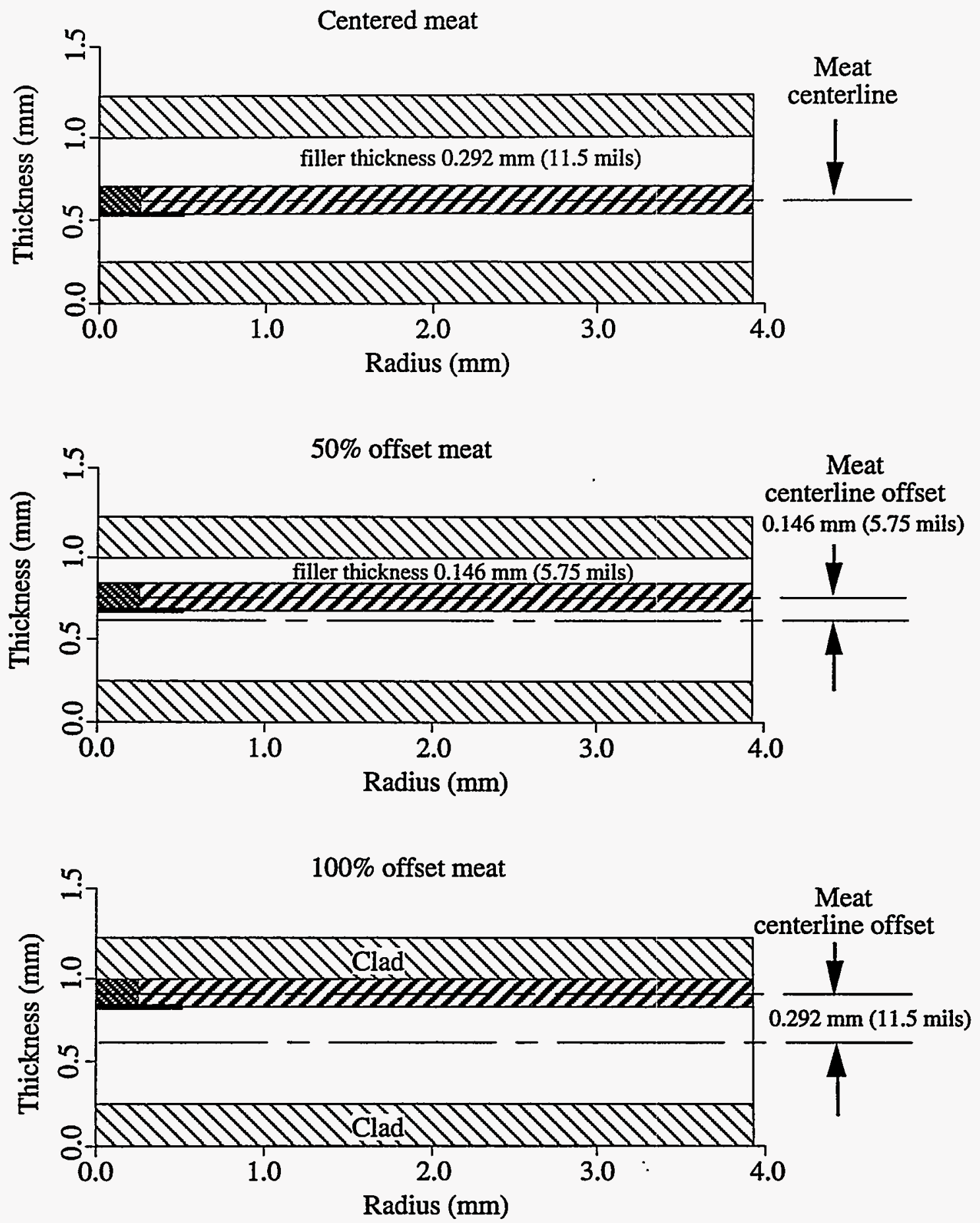

Fig. 3.16. Meat layer at centered, $50 \%$ offset and $100 \%$ offset positions. 


\section{LOCAL ANALYSIS TECHNIQUE}

The analysis techniques described in Sects. 2 and 3 are too conservative to allow the ANSR to generate the required neutron fluxes and experimental conditions. The Local Analysis Technique (LAT) was developed to relax some of the overly conservative assumptions made in the previous studies and provide a more accurate but still conservative technique to determine the reactor operating margins. The development of this technique was made possible by the emergence of more capable computing equipment. The limitations and improvements of these conservative assumptions are next discussed as an introduction to the LAT.

\subsection{DISCUSSION OF PREVIOUS ASSUMPTIONS:}

Assumption: Maximum relative power density occurs at all points and throughout the complete cycle. In reality, the power density varies with time and position. Figure 4.1 shows the calculated maximum and minimum relative power densities for the $\mathrm{L} 7$ core design as a function of time. The $35 \%$ reduction in maximum relative power density over the fuel cycle can produce a significant reduction in the fuel defect produced maximum temperatures.

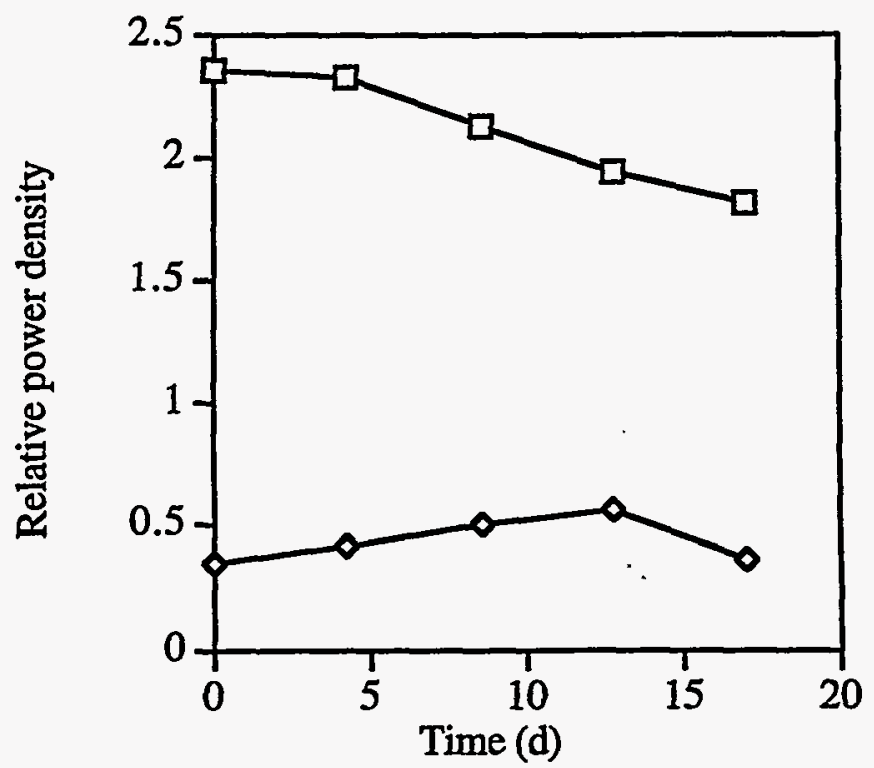

Fig. 4.1. Maximum and minimum relative power density as a function of time for the L7 core design. 
From this figure it can be inferred that a large portion of each fuel plate must have a relative power density much less than the maximum relative power density. The higher power densities exist in only a small portion of the plate as can be seen from Fig. 4.2. This figure shows the relative power density distributions for both $\mathrm{L} 7$ fuel plates at the first time step. Appendix D contains the relative power distributions for all time steps for the L7 core design.

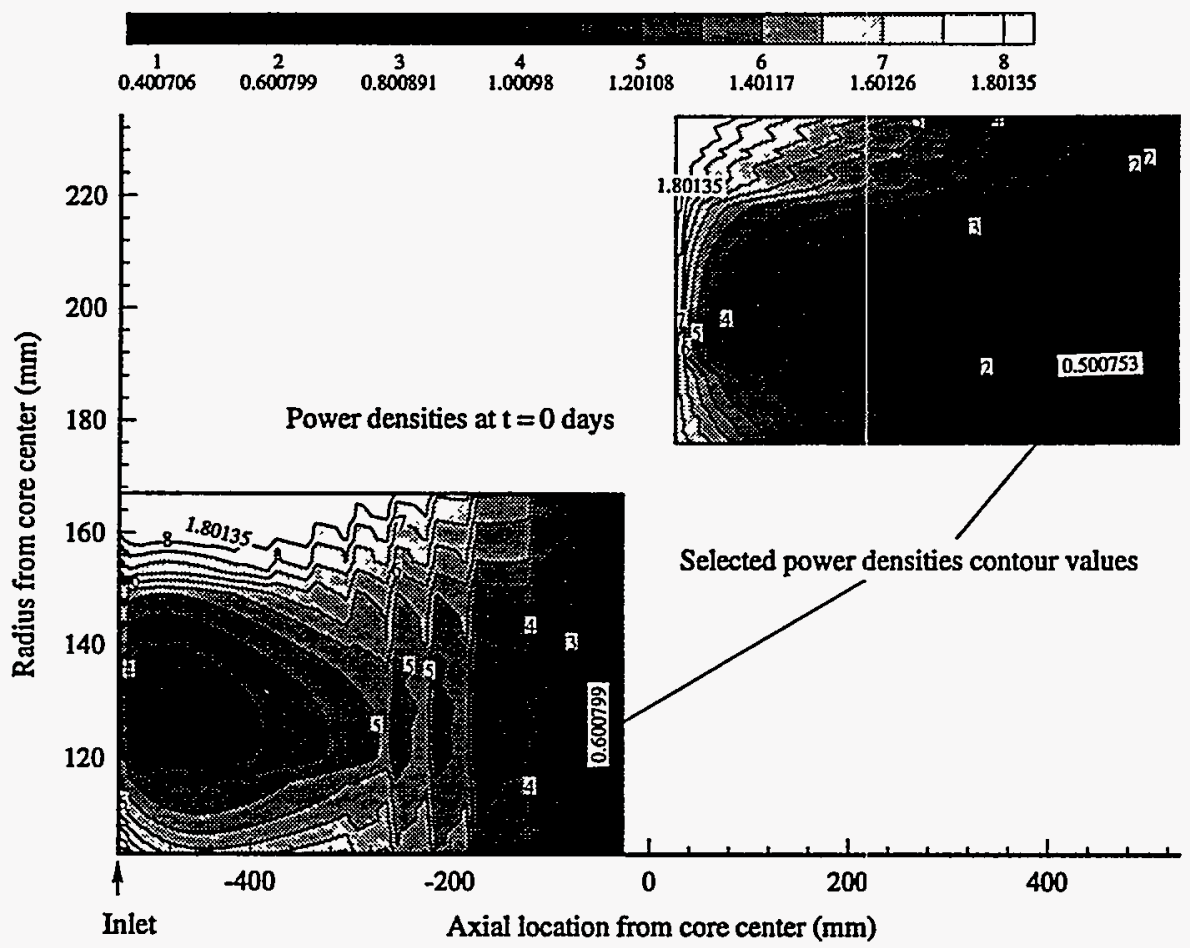

Fig. 4.2. Contours of relative power density on upper and lower fuel plates for the $\mathrm{L} 7$ core design, initial time step.

Assumption: Thermal conductivity is constant throughout the cycle and equal to the beginning-of-cycle (BOC) values for the normal and segregated fuel. Most of the previous analyses used the BOC values. The later analyses described in Sect. 3 used the EOC values. Section 3 discusses the variation of thermal conductivity of the normal and segregated fuel as the fission density increases throughout the radiation cycle. The fission density can be calculated from the VENTURE fuel burnup results. VENTURE only reports the local fuel burnup at the end of the cycle. In addition, the local fission density can be estimated from the local relative power density that is reported by VENTURE as a function of time. The technique for estimating the local time-dependent fission density is reported in Appendix E. Using the local fission density and the techniques discussed in Section 3, it is possible to estimate the thermal conductivities for every point in a specific design. 
Figure 4.3 shows the maximum and minimum conductivities for both normal and segregated fuel as a function of time for the L7 core design. As with the power density, there is a wide variation in these parameters across the fuel plates. Appendix D shows these data in detail.

In general, a reduced thermal conductivity will produce a higher maximum temperature. However, it is evident from comparison of Fig. 4.1 and 4.3 that the lower conductivity in normal fuel occurs only when the local power is relatively low. Also, it is not immediately apparent that the BOC or EOC thermal conductivities (used in most previous studies) are "strictly" conservative. The wide variation of conductivity during the cycle and the complex interaction with the thermal environment make it difficult to determine what single set of values represent the most conservative case.

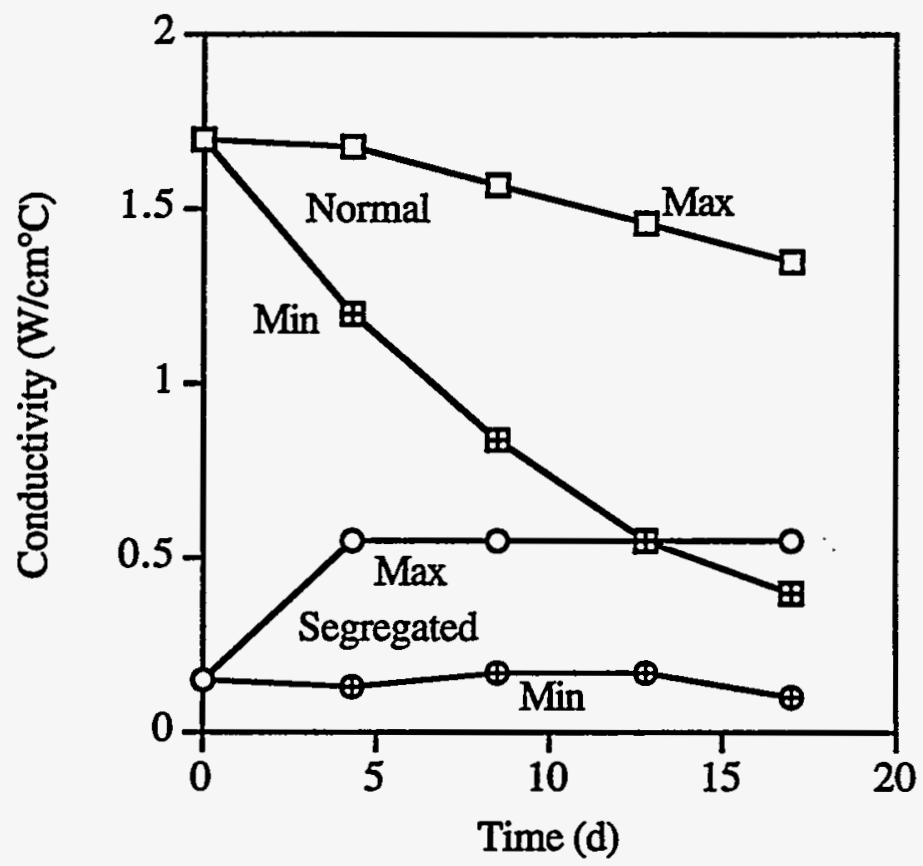

Fig. 4.3. Thermal conductivity of normal and segregated fuel as a function of time for the L7 core design.

Assumption: The same oxide thickness occurs at every point throughout the cycle. Previous calculations used either zero or the maximum oxide layer thickness. The maximum oxide thickness is set by design limits to be $30 \mu \mathrm{m}$ which is larger than that found in the L7 core design TASHA calculations. Figure 4.4 shows the maximum and minimum thickness as a function of time. Note the wide variation in oxide thickness at all but the first time step. Appendix D shows these data in detail. 
As shown in Sect. 3, the oxide layer thickness affects the peaking factor and maximum temperature in opposite manners. The most conservative assumption on oxide thickness for the peaking factor calculation is no oxide; the most conservative assumption for the maximum temperature calculation is the maximum oxide thickness. The previous analyses performed calculations with zero and the maximum thickness and selected the conservative values for the peaking factor and the maximum temperature.

Assumption: Coolant temperature is constant for all time and locations and equal to the bulk averaged exit temperature $\left(90^{\circ} \mathrm{C}\right)$. The maximum and minimum coolant temperatures as a function of time is shown in Fig. 4.5. The hottest coolant is near the exit: the higher power regions are near the inlet.

Assumption: The meat thickness varies from minimum to maximum for all the above conditions. In the L7 core design the meat thickness varies from 0.2961 to $0.7366 \mathrm{~cm}$. The meat thickness distribution over the L7 fuel plate is shown in Appendix D.

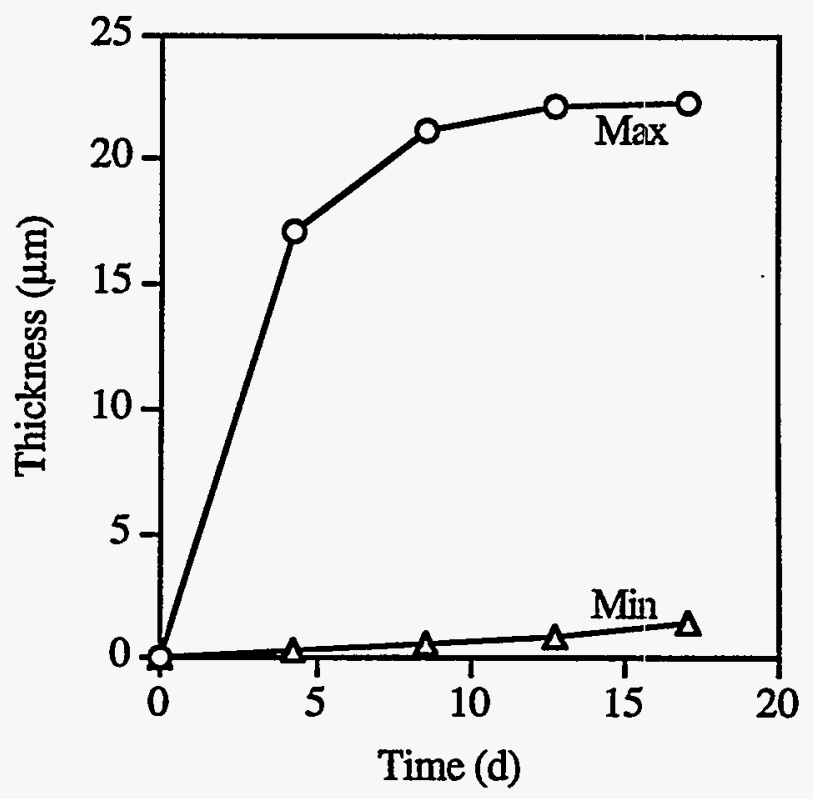

Fig. 4.4. Maximum and minimum oxide layer thickness as a function of time for the L7 core design.

A súbstantial and complex variation is noted in these parameters during the cycle and across the plate. The complexity of the interaction of these parameters makes it difficult to determine a unique set of strictly conservative values without excessive conservatism. The development of the LAT is an attempt to improve on this situation. 


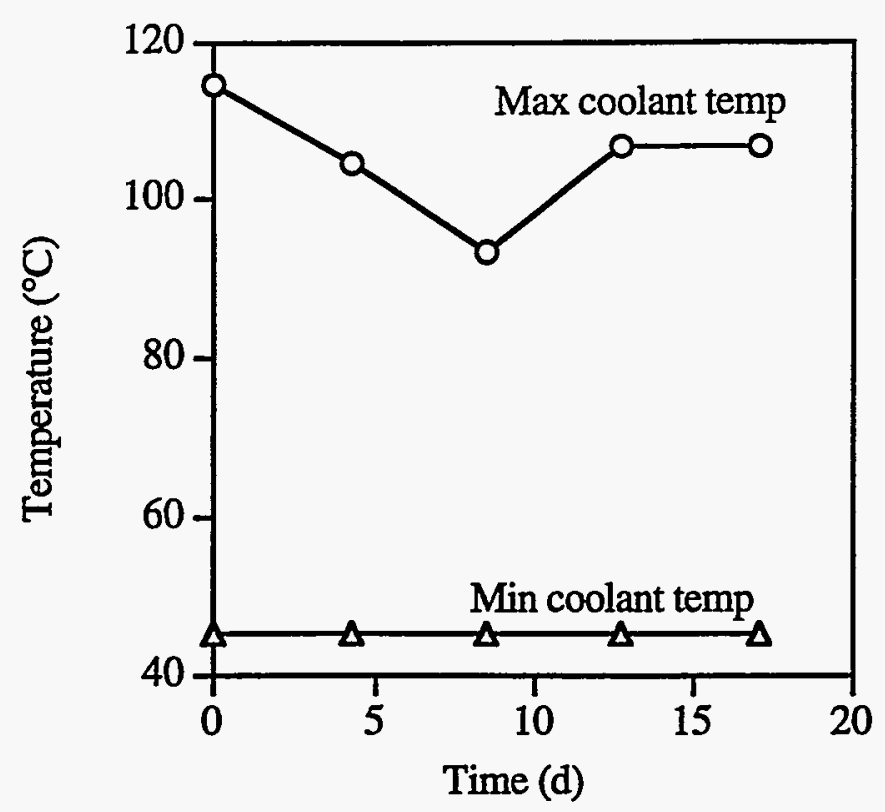

Fig. 4.5. Maximum and minimum coolant temperature as a function of time for the L7 core design.

\subsection{IMPROVED ANALYSIS TECHNIQUE}

How can we provide a more accurate analysis technique that remains conservative? Relaxing just one of the previous assumptions might provide sufficient margin to allow design goals to be met. This might be done by varying one parameter over time while the other parameters were fixed at conservative values. However, it was unclear what the interaction of the complicated thermal parameters would be. It is possible that the simplified analysis where just one parameter was varied over time might not be accurate or conservative enough. A more accurate analysis incorporating the interactions between these complex phenomena might produce worse results.

For example, one approach might be to use the maximum relative power density for each time step in a meat thickness variation analysis while fixing the other parameters at EOC values. Because the thermal conductivity varies over a large range during the reactor cycle, there is some uncertainty that the EOC values are conservative for the complete cycle. Similar doubts arise concerning the heat transfer coefficient, coolant temperature and oxide layer thickness. Not being able to make convincing arguments for remaining conservative precluded using this simpler approach. It may be possible to make such an argument. However, further development in this area was made unnecessary because of the existence of sufficient computational 
power and tools to allow a more detailed analysis technique to succeed. Even if a simplified analysis could be developecl and defended, it would produce more conservative results and might therefore be more limiting on the ANSR operation.

The approach chosen for this development was to take the current local conditions for every time step and location on the fuel plate. These local conditions were applied to a 2-D coincident nonbond and segregation defect model at each point on the fuel plates. This approach is the mathematical equivalent of moving an upper-limit defect pair around the plate to determine the worst possible defect location under the local conditions.

The LAT reduces the number and scope of arguments required to defend the conservatism of an assumption by using a large number of analyses to encompass the known (calculated) conditions on the fuel plate.

The points chosen for this study were those used in the VENTURE calculations. Two grids were used in those calculations;. (1) that used by VENTURE - a detailed two-dimensional ( $\mathrm{rz}$ ) neutronics calculation (Fig. 4.6), and (2) the meat thickness design data base grid (Fig. 4.7). The points in these grid are spread over the portion of the plate that contain fuel. The finer set of points, those for the VENTURE neutronics calculations, was chosen as the grid for the LAT.

The local conditions for each point on the plates were taken from the appropriate data base. There are three pertinent data bases. The meat thickness design data base, the VENTURE produced data base and the Thermal Analysis of $\underline{\text { Steady-State }} \underline{\text { Heat Transfer for }}$ ANSR ${ }^{10}$ (TASHA) produced data base.

The meat thickness design data base was developed by the core designers to meet the various constraints (power level, total fuel weight, etc.) and used as input into the VENTURE code. Calculated VENTURE power density load was used as input to the TASHA code. The results of these analyses are used to determine if the design meets all the constraints. For the LAT, the meat thickness data were interpolated onto the VENTURE grid.

VENTURE analyzes the neutron production (by fission), reflection and absorption within the ANSR core design and produces a data base of the relative power density as a function of time and location. The local relative power density from this data base was used directly to determine the local heat generation for the normal and segregated fuel in the LAT 2-D thermal models. The local relative power density was integrated to estimate the local fission density, discussed in Appendix E. The fission density was used to calculate the local time-dependent fuel concluctivities (normal and segregated) as discussed in Sect. 3.

TASHA uses time- and space-dependent relative power density distributions and estimates of uncertainty factors in a set of idealized 1-D 
thermal conduction and convection models to provide a conservative estimate of the worst possible local thermal conditions in the fuel and coolant. TASHA produces a data base containing the estimates of the local maximum temperature in normal fuel, coolant temperature, oxide layer thickness, and other thermal parameters.

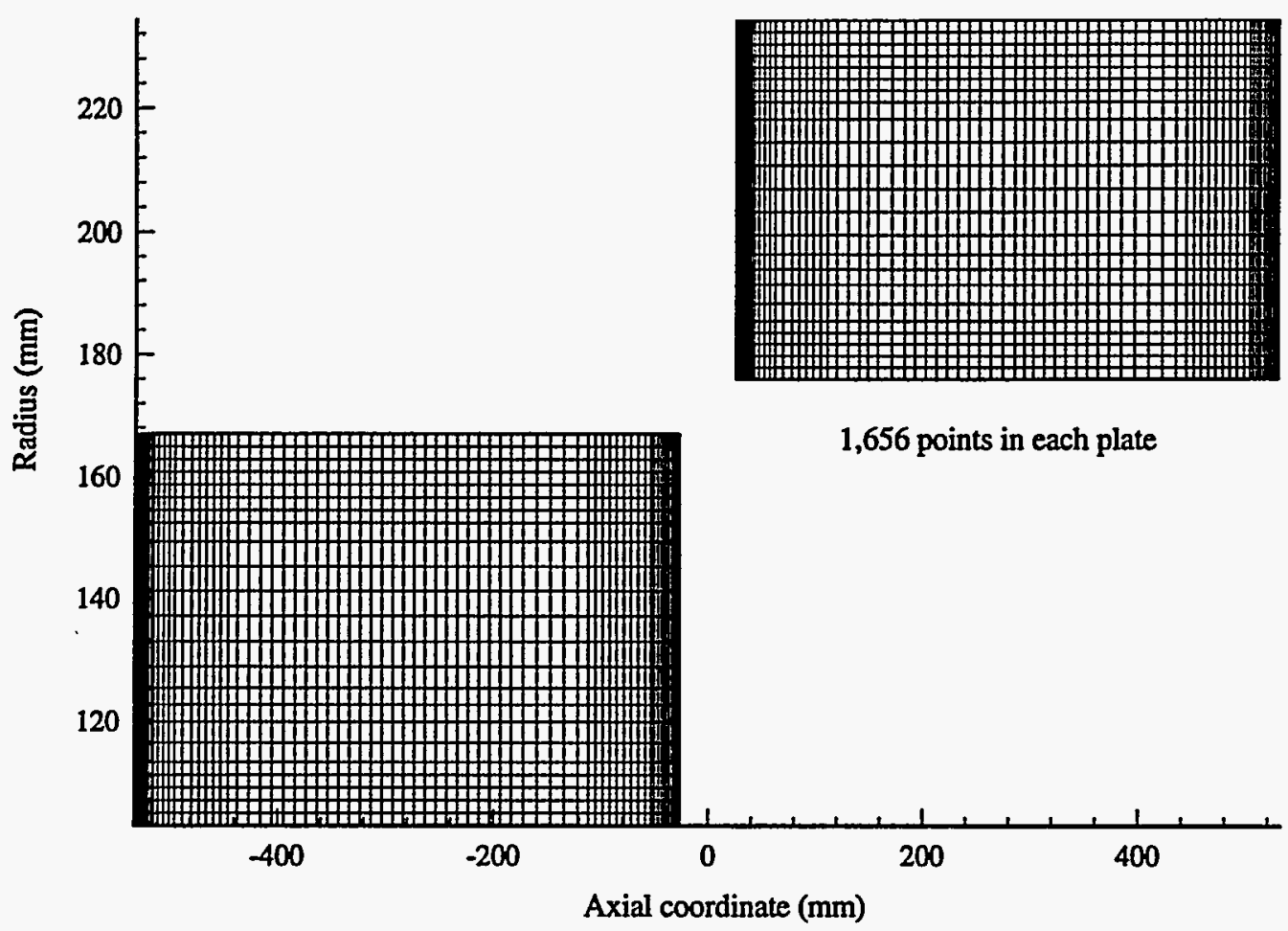

Fig. 4.6. Grid from VENTURE analysis of ANSR L7 fuel plates.

TASHA coolant temperature results are conservative. The code uses an uncertainty factor for the coolant temperature that accommodates the possible existence of a hot streak along a coolant flow line. Because of the locally elevated surface heat flux, coolant temperatures can be increased above what would be experienced with only normal fuel. The inspection procedure allows a hot streak that lies in a 12.7-mm-long, 0.4-mm-wide stripe that can produce a $10 \%$ increase in the local coolant temperature. This factor is included in the TASHA model. Therefore, the TASHA estimated coolant temperatures are higher than would be expected if no defects were present. 


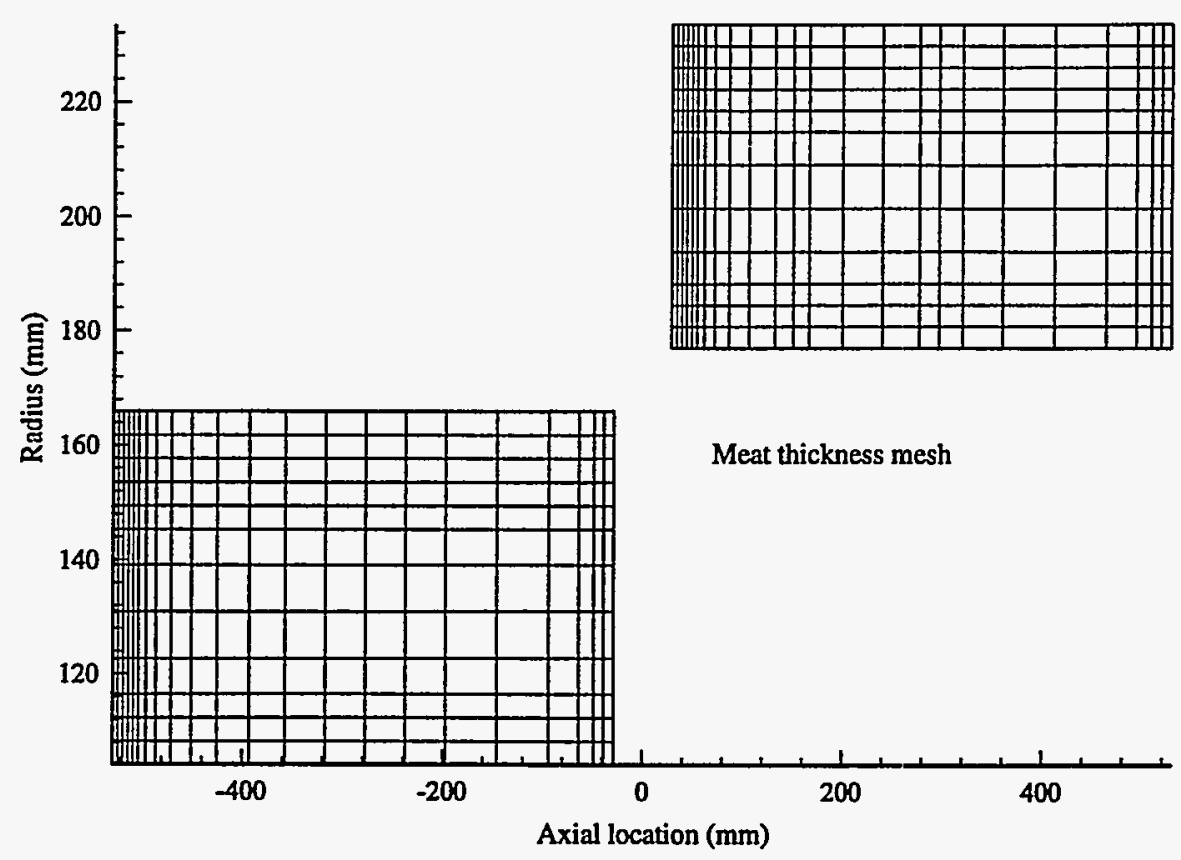

Fig. 4.7. Meat thickness design grid for ANSR L7 fuel plates.

The oxide layer growth is estimated in TASHA by using a somewhat conservative estimate of the local heat flux. The local heat flux, which is used to calculate the oxide layer thickness, is modified by the hot streak uncertainty factor of 1.10. However, the true local peaking factor (in an area less than $2 \mathrm{~mm}$ in diameter) can be higher than 1.10. As seen in Sect. 3, thicker oxide layers tend to increase the maximum fuel temperature but tend to decrease the maximum peaking factor. The TASHA oxide layer thicknesses are intended to conservatively estimate the maximum temperatures in the normal fuel. When used in the LAT they present a compromise in conservatism between maximum temperature and peaking factors.

The TASHA local coolant temperatures and oxide layer thicknesses are used in the local thermal analysis of the defect pair model to produce an estimate of the maximum fuel temperature and peaking factor at each point for each time step. However, TASHA uses some additional conservative assumptions that complicate the direct application of results. The power density at the beginning of the time step is used in TASHA to grow an oxide layer for the whole step. It then uses the power level at the beginning of the time step with the oxide at the end to calculate the maximum temperatures for the time step. With the exception of the early part of the cycle, the maximum power density at a point is generally dropping. Thus, this assumption is generally conservative in maximum temperature for all but the 
first part of the cycle. There are points on the fuel plates that increase in power later in the cycle. However, these points are generally lower in power and would produce temperatures lower than the earlier maximums. Still, this TASHA approximation is not strictly conservative at these points.

\subsubsection{Description of Model}

The basic model used in the LAT is the same 2-D axisymmetric coincident nonbond-segregation defect pair model described in Sect. 3. The local coolant temperature, oxide thickness, relative power density, meat thickness and conductivities are used in a series of calculations. Each input file describing the model at a point is automatically generated, analyzed by HEATING, and the results accumulated into a common file for postprocessing. The code that automatically performs this series of analyses is described in Appendix A.

Because of the detailed nature of the LAT it is possible to incorporate a more complex model of the thermal conductivity of the segregated fuel. This model is required by the complex chemical and thermodynamic interaction within the fuel under irradiation and is discussed in Sect. 7.

\subsubsection{Uncertainty Levels Used In The Analysis}

The LAT was used to perform nominal analysis where all thermal parameters were taken at the nominal value. The coolant temperatures and oxide thicknesses were taken from a TASHA "Best Estimate" analysis. In this type of TASHA analysis only hot streak (1.1) and hot spot (1.31) uncertainties are applied. This set of conditions is referred to as a nominal analysis in this report.

To provide confidence in the operation of the reactor, analyses using the TASHA code are performed. One type of these analyses is called a 95\% probability case. This analysis uses a set of parameters that represent the 95\% probability level for the major uncertainties. Some of these parameters include the coolant channel thickness $(-10 \%)$, power level and distribution uncertainty factors $(6 \%)$, heat transfer coefficient uncertainty $(-6 \%)$, and uncertainties due to fuel extending beyond the normal fuel boundary. To perform a 95\% LAT analysis these uncertainties were used in the TASHA code to calculate the oxide thickness and coolant temperatures. These same uncertainties were used in the LAT analysis to calculate the local heat transfer coefficient and heat generation. All other thermal parameters taken at nominal values.

The TASHA code has been used with another set of uncertainties to calculate the thermal limits for conditions at the limiting safety system setpoint (LSSS). The LAT has not yet been used for this type of analysis. 


\subsection{INVESTIGATIONS OF NOMINAL VALUES USED IN THE LAT}

The LAT uses calculated values as input. Some of these values can be generated by TASHA analyses that account for the probabilities of variations in these values to be expected in the real world. TASHA can incorporate uncertainties for the hot spot, hot streak, power level and distribution, and narrow channel conditions. These results are used in the LAT only for coolant temperature and oxide thickness. Fission densities and thermal conductivities have no uncertainties applied. Uncertainties in these parameters must be accounted for in the application of the LAT to design and safety analysis.

Although limited investigations of these uncertainties have been performed, more extensive investigations should be done. These investigations may require additional uncertainties to be applied to the LAT results. Also, in order to accommodate these uncertainties the LAT may have to be modified to perform two or more analyses at each point. Each separate analysis will use the local thermal conditions with different assumptions that are designed to be conservative for either the peaking factor or maximum fuel temperature. More than two analyses may be needed at each point because it may not be possible to determine a strictly conservative set of conflicting assumptions.

\subsubsection{Thermal Conductivity}

One investigation of the effects of uncertainties on the thermal conductivity was done for the L7 core design. In this study an early version of the LAT was used to produce the maximum temperatures for the EOC conditions only. The thermal conductivities of the normal and segregated fuel was varied by $\pm 10 \%$ from the nominal value calculated from the fission density at the end of the cycle. At each data point, five analyses were performed. The first analysis was for the nominal values. The second used the $+10 \%$ conductivities. The third uses the low $(-10 \%)$ conductivities. The fourth used $+10 \%$ for the normal fuel and $-10 \%$ for the segregated fuel. The final analysis used the $-10 \%$ for the normal and $+10 \%$ for the segregated fuel conductivity. This permutation process was used because it was not clear which set of parameters would be the worst condition in all cases. This variation produced only a $3.5 \%$ increase in the maximum temperature. Therefore, it was concluded that the maximum temperatures are affected by the thermal conductivity but that it was not overly sensitive to them.

\subsubsection{Oxide Layer Thickness Growth Model}

The exact oxide layer thickness at any time is not known precisely. TASHA estimates were used in the LAT initially. LAT calculates the local maximum heat flux and clad-oxide interface maximum temperature for each 
hot spot. The LAT was modified to use this local information to more conservatively calculate the local oxide layer thickness and local maximum temperature. The LAT-produced oxide thicknesses were $28 \%$ larger than TASHA's because the local maximum surface heat flux was used. Even with this more conservative oxide layer, the maximum temperatures were only slightly higher, $\sim 3 \%$.

The oxide growth correlation used in TASHA and LAT was developed from experiments on plates that were maintained at uniform heat fluxes for different of coolant temperatures. It is not known if non-uniform local heat fluxes (i.e. local hot spots) will produce oxide growth rates similar to the experiments. The TASHA assumption of using the hot streak uncertainty factor of 1.1 on the heat flux is equivalent to assuming that the heat flux over a certain area is the controlling parameter rather than the local or peak heat flux. The LAT analyses assumed that the oxide growth was controlled by the peak heat flux and the oxide layer was of a uniform thickness over the surface of the model. Both TASHA and the current LAT assume that the oxide layer is the same on both the hot and cold surfaces. It is likely that the hot and cold side oxide layer will be of different thicknesses. Although, it is possible to simulate these more sophisticated oxide growth models in the LAT, the development was halted before completion.

\subsubsection{Time Discretization}

The LAT uses the same time discretization as the VENTURE calculations. The VENTURE time discretization used for the L7 core design is fairly coarse. Limited investigations of interpolating between the VENTURE time steps indicate that early time steps may not be fine enough to calculate the maximum temperature and peaking factor.

These investigations used the VENTURE and TASHA results and linearly interpolated between the time steps for the local power density, fission density, coolant temperature and oxide thickness. A linear interpolation for such complex phenomena as the production of nuclear heating or production of fissioned nuclei is a very crude approximation. This approximation is taken only because of the lack of other data, and it gives only a limited indication of the true response of the thermal system. The results did indicate that the early time step of 4.25 days was not sufficient. For later VENTURE calculations a 1.0-day initial time step was used.

\subsubsection{Spatial Discretization}

It is conceivable, although unlikely, that in between the spatial data points picked for analysis there could exist conditions that could produce slightly greater maximum temperatures and peaking factors. The effect of this uncertainty is thought to be quite small and was not investigated. 


\subsection{ADVANTAGES OF THE LAT}

The LAT can provide the following advantages:

1. Provides assurance that maximum temperatures will be less than the extreme temperatures calculated in the previous strictly conservative analysis,

2. Provides assurance that the actual peaking factors are much lower than other analyses indicate,

3. The calculations can be implemented in a Monte Carlo thermohydraulics code,

4. Allows the development of a multidimensional functional representation of the results,

5. Allows the use of a more sophisticated model for the thermal conductivity and oxide growth model than the TASHA code, and

6. Reduces reliance on assumptions of conservatism that are difficult to defend based on more detailed understanding the fuel thermal properties. 


\section{L7 CORE DESIGN LAT RESULTS}

The LAT was applied to the L7 core design. The five time steps of the VENTURE calculation required 16,740 separate HEATING analyses. Even though each analysis only takes a few seconds of computing time on an IBM RISC 6000-580 computer, the complete series still takes several hours.

After the series is finished, the data base containing the results is large enough to offer some challenges in understanding. The results of an analysis using the TASHA 95\% probability case for the L7 core design are first presented here for comparison with the earlier meat thickness variation analyses. Thus Fig. 5.1 presents all of the peaking factors calculated for the complete cycle on one plot as a function of the local meat thickness. Figure 5.2 shows the maximum temperature for all the data points as a function of meat thickness. The results for the design limits analyses are included on these figures. All of the many data points from the LAT are below those from the design limits analyses and most are below the L7 EOC worst-case value meat thickness analyses of Sect. 3. The fact that the values exceed the L7 EOC limits demonstrates the nonconservatism of determining values from the EOC conditions only.

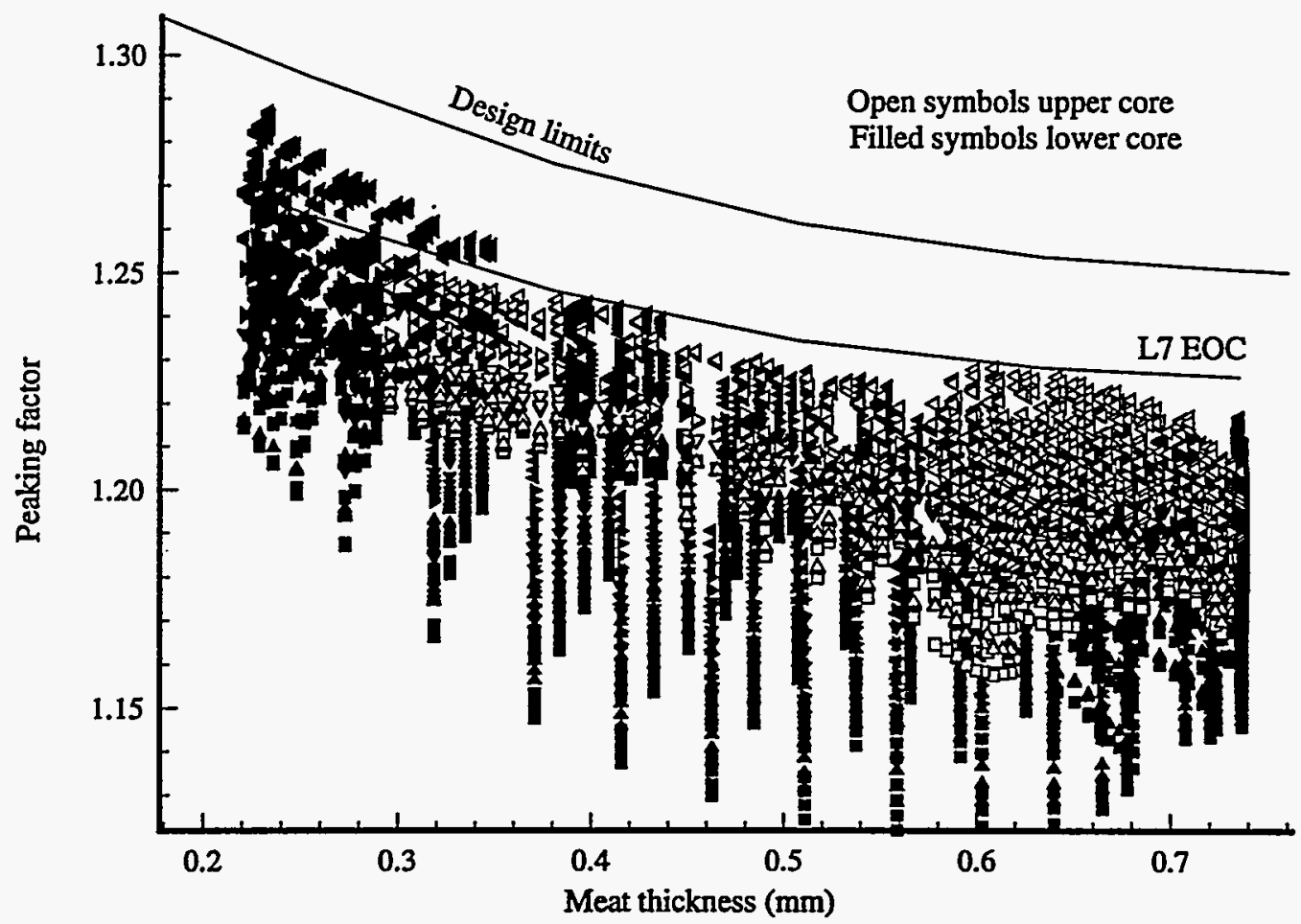

Fig. 5.1. Peaking factor results from the LAT analysis of the L7 core design compared with design limit results as a function of meat thickness. 


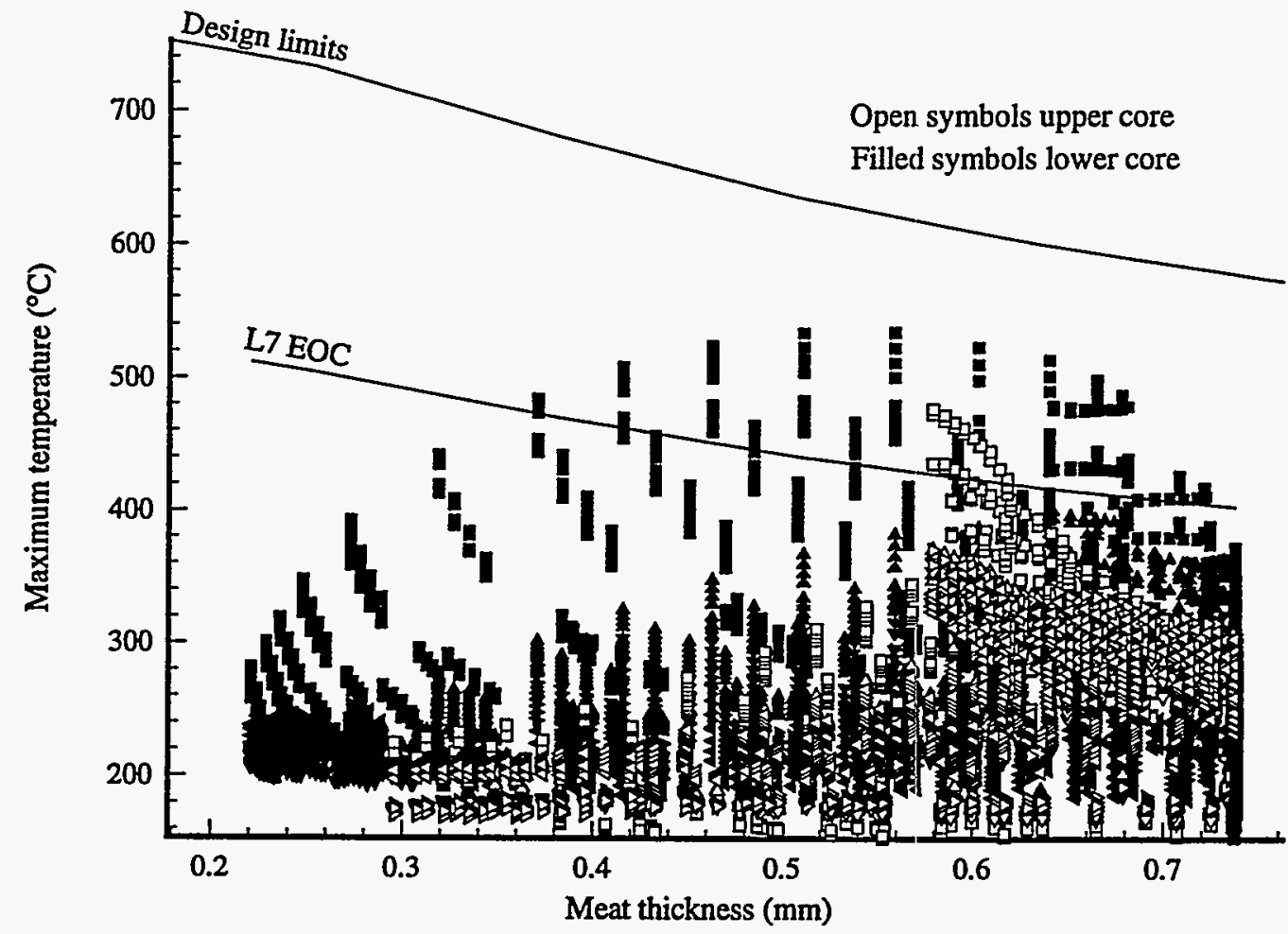

Fig. 5.2. Maximum temperature results from the LAT analysis of the L7 core design compared with design limit results as a function of meat thickness.

The differences between the peaking factor and maximum temperature results are significant. If the maximum values of the LAT results were used instead of the maximum design limit values the benefits would be about $6 \%$ to $21 \%$ in peaking factor and about $37 \%$ to $42 \%$ in maximum temperature. One approach that would be simpler than the LAT would be to use the design limit value as a function of local meat thickness. This approach is more conservative than necessary by at least 3\% in peaking factor and $14 \%$ in maximum temperatures. The maximum tenaperatures of the meat thickness variation analyses and the LAT analysis all exceed $400^{\circ} \mathrm{C}$ within the segregation volume.

The data summarized as a function of time are shown in Figs. 5.3 and 5.4. The large reduction in peaking factor early in the cycle can provide substantial improvement in operating conditions for the ANSR, because the most limiting condition usually occurs early in the cycle when the power density is largest. Also, the growth of the oxide layer is greatly influenced by the local heat flux. Reducing the peaking factor would produce thinner oxide layers than predicted by TASHA. A thinner oxide layer will reduce the 
maximum temperatures in the current and later time steps. In these calculations, the maximum temperature for the first time step exceeds $400^{\circ} \mathrm{C}$. The TASHA-produced overestimate of the oxide thickness was used in these calculations. More accurate estimates would produce lower maximum temperatures. Even with the TASHA overestimation in oxide thickness, the area of the plate that exceeds the limit is small, as will be shown below.

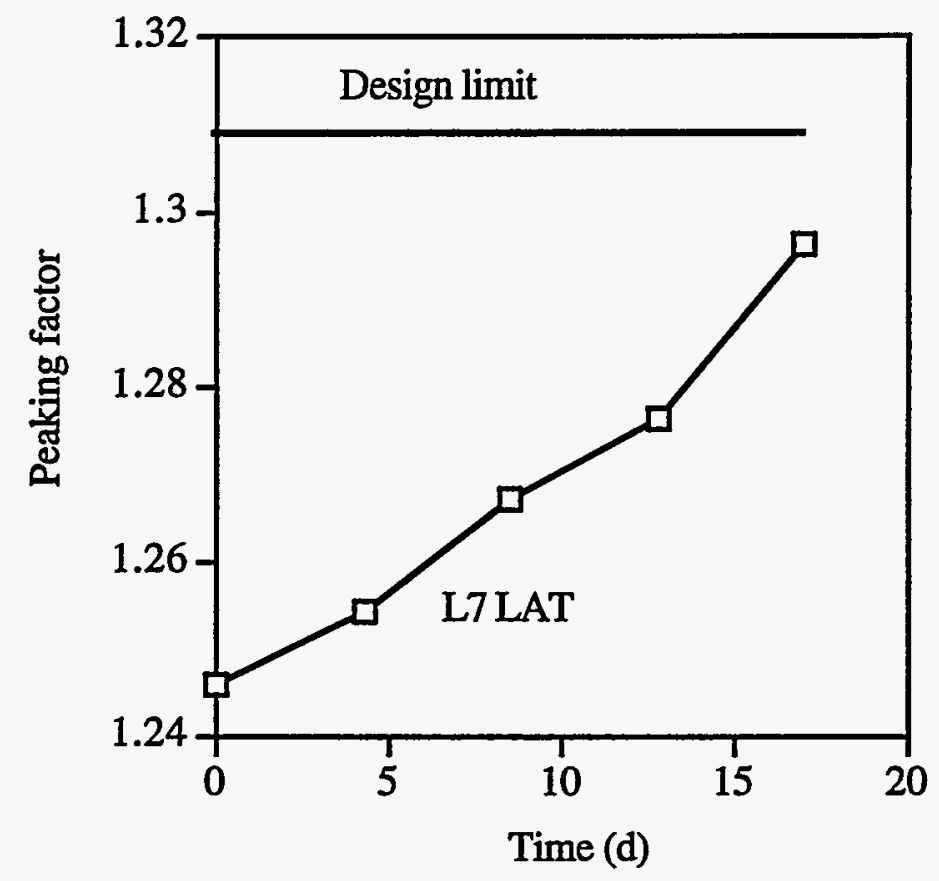

Fig 5.3. Maximum peaking factors from L7 core design LAT analysis compared with the design limit results.

Another method for visualization of the LAT results is to present them as contours on the fuel plate surface. For this type of presentation, the fuel plate is represented as a flat plate. The curved surface is represented as if it were flattened out. Figure 5.5 presents the contours of the LAT-produced peaking factor for the first time step for both plates of the L7 core design. Figure 5.6 shows the maximum temperature distribution on the surface of the fuel plates. Figures 5.7 and 5.8 show how small the areas of the plates are that exceed the thermal limits. Figure 5.7 presents the temperature results in a carpet plot where the height above the $\mathrm{rz}$ plane is determined by the local maximum temperature. Only a small region near the lower outer corner of the top plate exceeds the limit. On the bottom plate, only a thin stripe along the outer edge exceeds the limit. The rest of the area of the two plates is mostly well below the limit. Figure 5.8 shows the temperature contours on a representation of the plates drawn to scale to give the true proportion of the region that exceeds the thermal limit. 


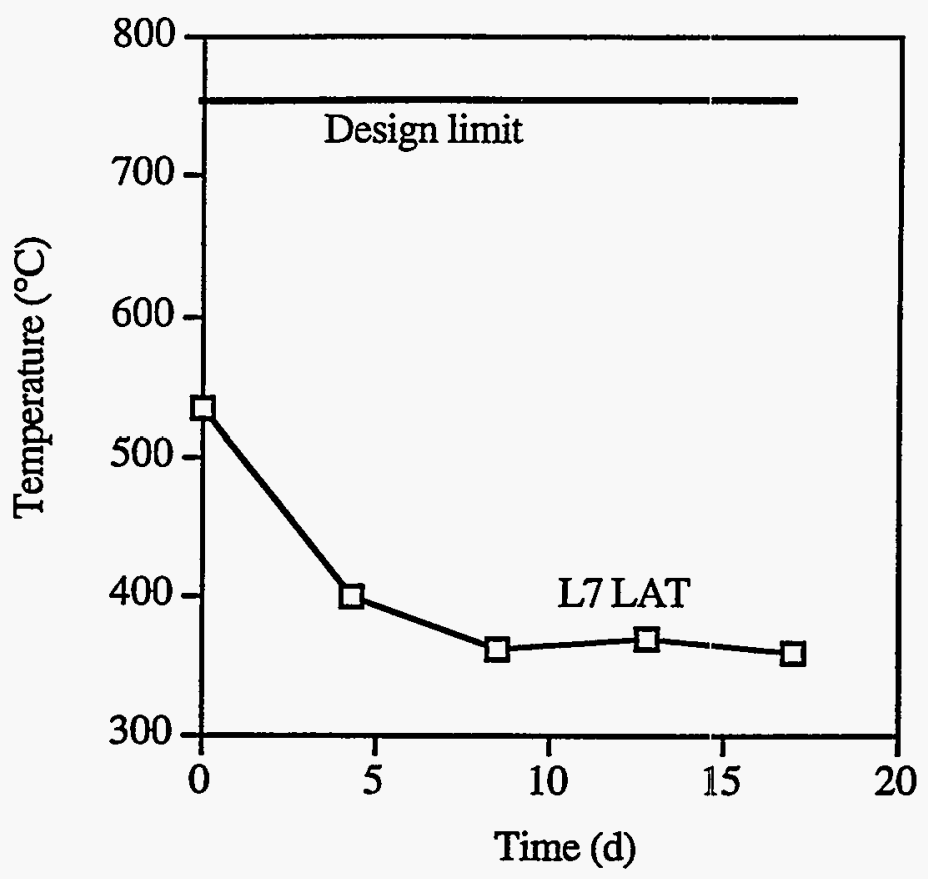

Fig 5.4. Comparison of L7 maximurn temperatures with design limit analysis.

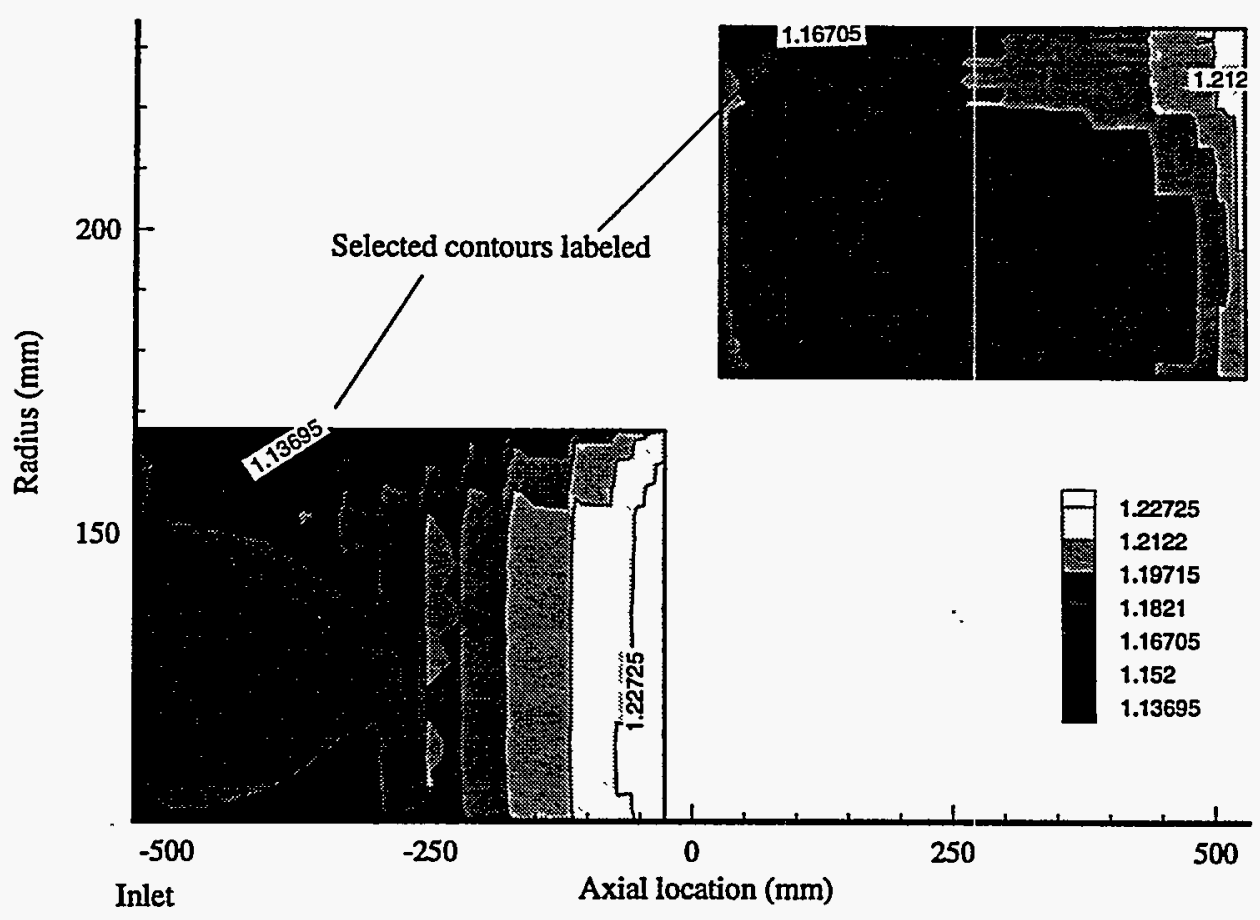

Fig. 5.5. Contours of peaking factor on L7 fuel plates. First time step of LAT analysis. The radius scale is exaggerated for clarity. 


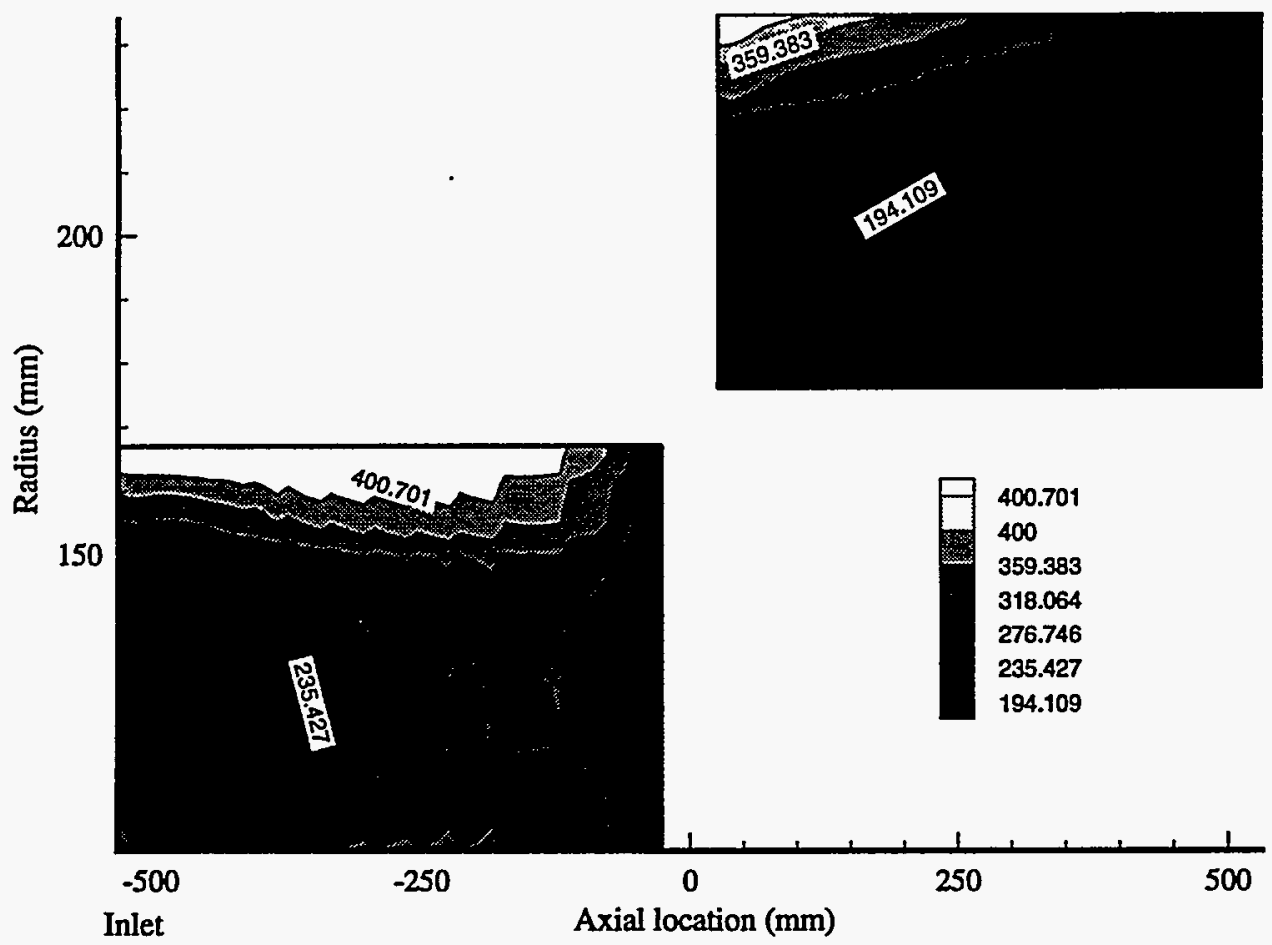

Fig. 5.6. Contours of maximum temperature in the segregation volume on L7 fuel plates, first time step of LAT analysis.

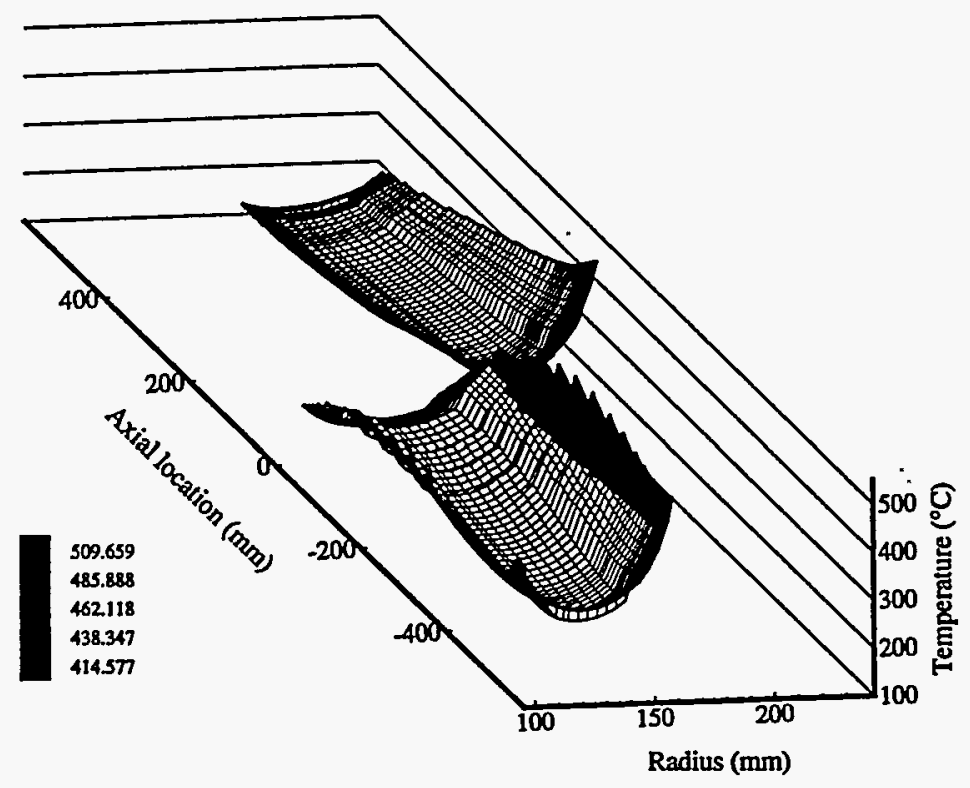

Fig. 5.7. Carpet plot of maximum temperature in the segregation volume on L7 fuel plates, first time step. Shaded region exceeds $400^{\circ} \mathrm{C}$. 


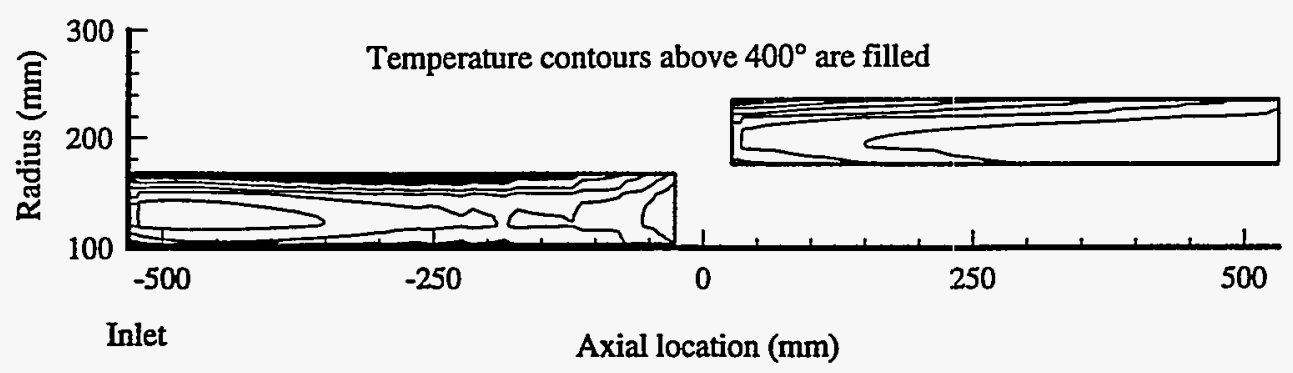

Fig. 5.8. Contour plot of maximum temperature in the segregation volume on L7 fuel plates drawn to scale. First time step of LAT analysis. Shaded region exceeds $400^{\circ} \mathrm{C}$. 


\section{G693 CORE DESIGN LAT RESULTS}

The results of the $\mathrm{L} 7$ thermal hydraulic analysis, together with other design constraints and modifications prompted the development of a new core design, G693. The LAT G693 core design nominal analysis results are first presented here for comparison with the earlier meat thickness variation analyses. Thus Fig. 6.1 presents all of the peaking factors calculated for the complete cycle on one plot as a function of the local meat thickness. Figure 6.2 shows the maximum temperatures for all the data points as a function of meat thickness. The results for the design limits and L7 EOC analyses are included on these figures. All of the many data points from the LAT are below the design limit and L7 EOC analyses. Comparing Figs. 5.1 and 5.2 with 6.1 and 6.2 show that the G693 core design improved the local peaking factors substantially. This new core design significantly reduced the temperatures on most of the plates. However, the maximum temperature in the segregation volume produced by this design is nearly the same as the previous design.

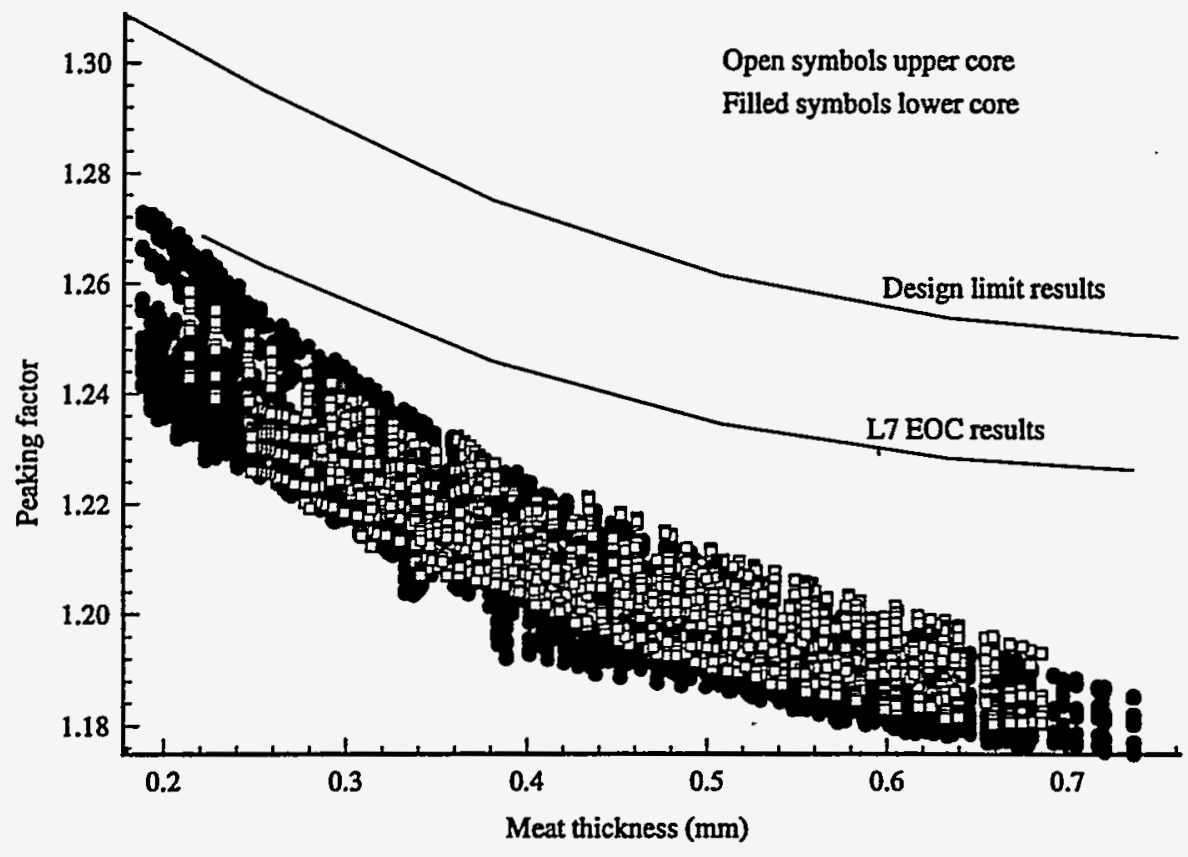

Fig. 6.1. Peaking factor results from the LAT analysis of the G693 core design compared with design limits results. 


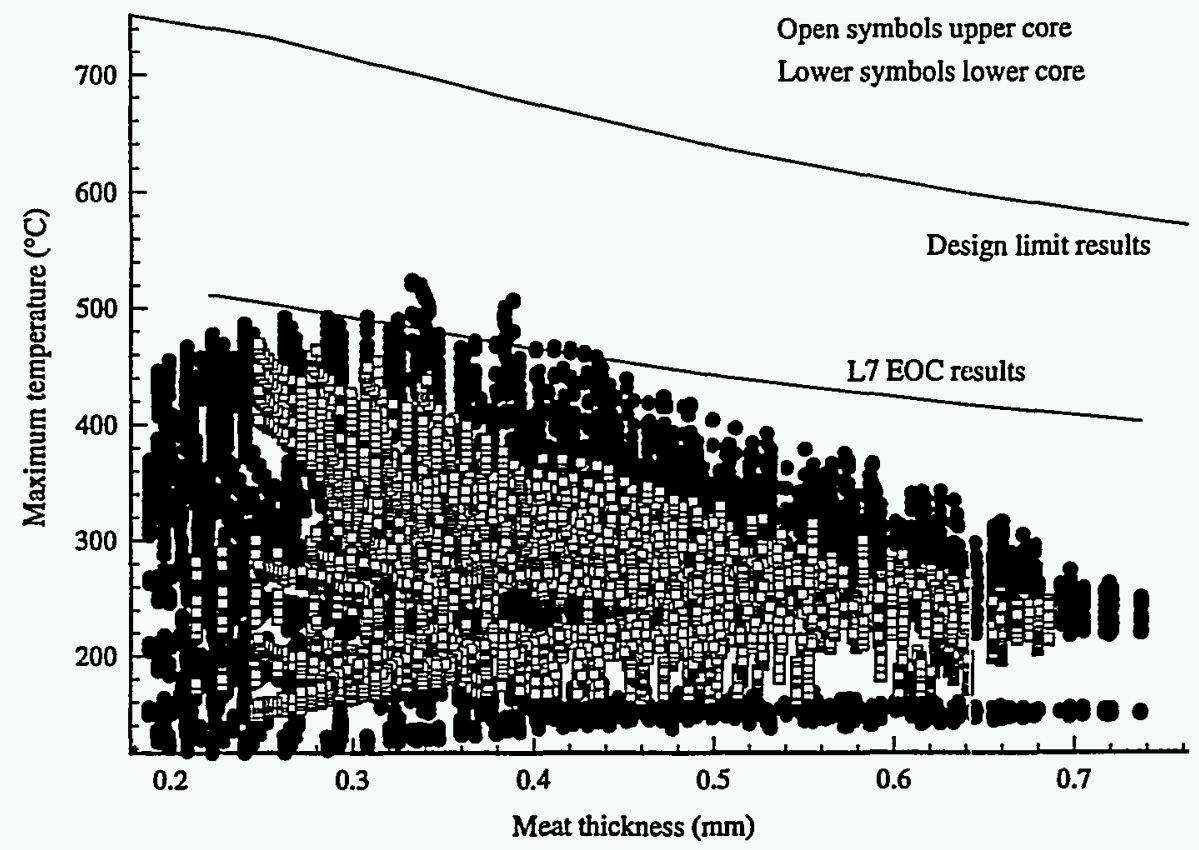

Fig. 6.2. Maximum temperature results in the segregation volume from the LAT analysis of the G693 core design compared with design limits results as a function of meat thickness.

The differences between the peaking factor and maximum temperature results are significant. If the maximum extent of the LAT results were used instead of the maximum design limit values the benefits would be about $3 \%$ in peaking factor and about $30 \%$ in maximum temperature. If the design limit values as a function of local meat thickness are used in a meat thickness variation analysis it would be more conservative than necessary by at least $3 \%$ in peaking factor and $25 \%$ in maximum temperatures. The maximum temperatures in the segregated fuel of the G693 LAT still exceed $400^{\circ} \mathrm{C}$.

The data summarized as a function of time are shown in Fig. 6.3 and 6.4. The maximum segregated fuel temperature for the first three time steps exceeds $400^{\circ} \mathrm{C}$. Although the area of the plate that exceeds $400^{\circ} \mathrm{C}$ in the G693 design is larger than the L7 design it is still a small portion of the plate area. 


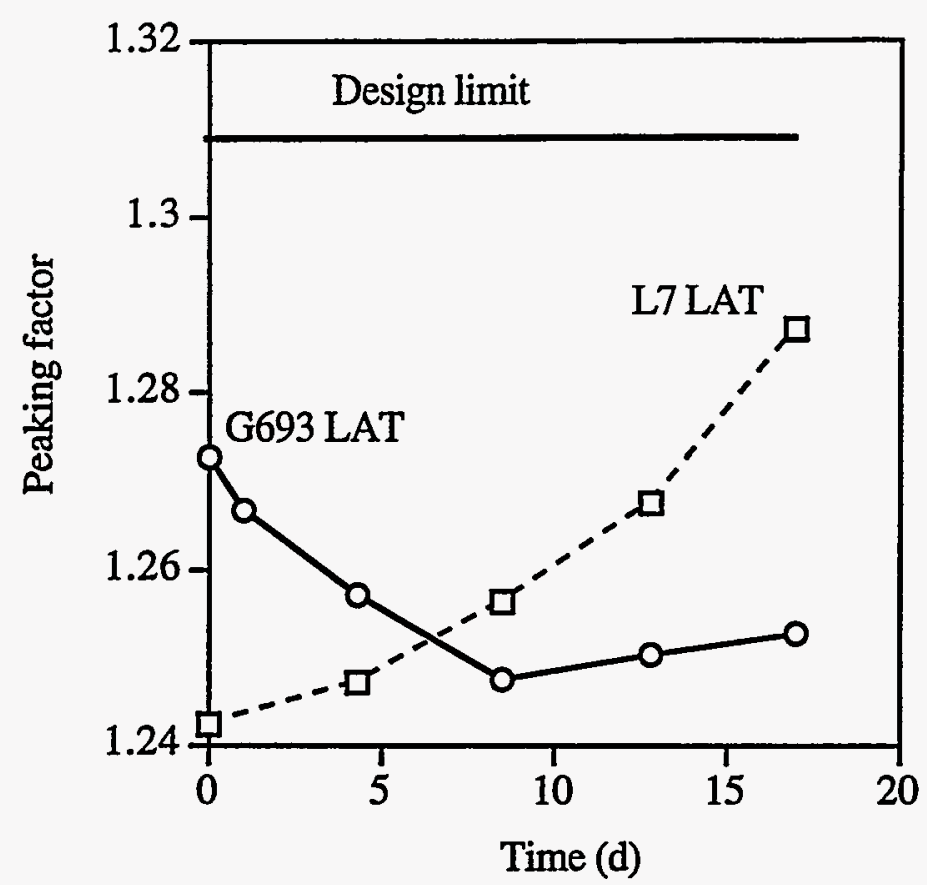

Fig. 6.3. Maximum peaking factors from G693 core design LAT analysis compared with the design limit results as a function of time.

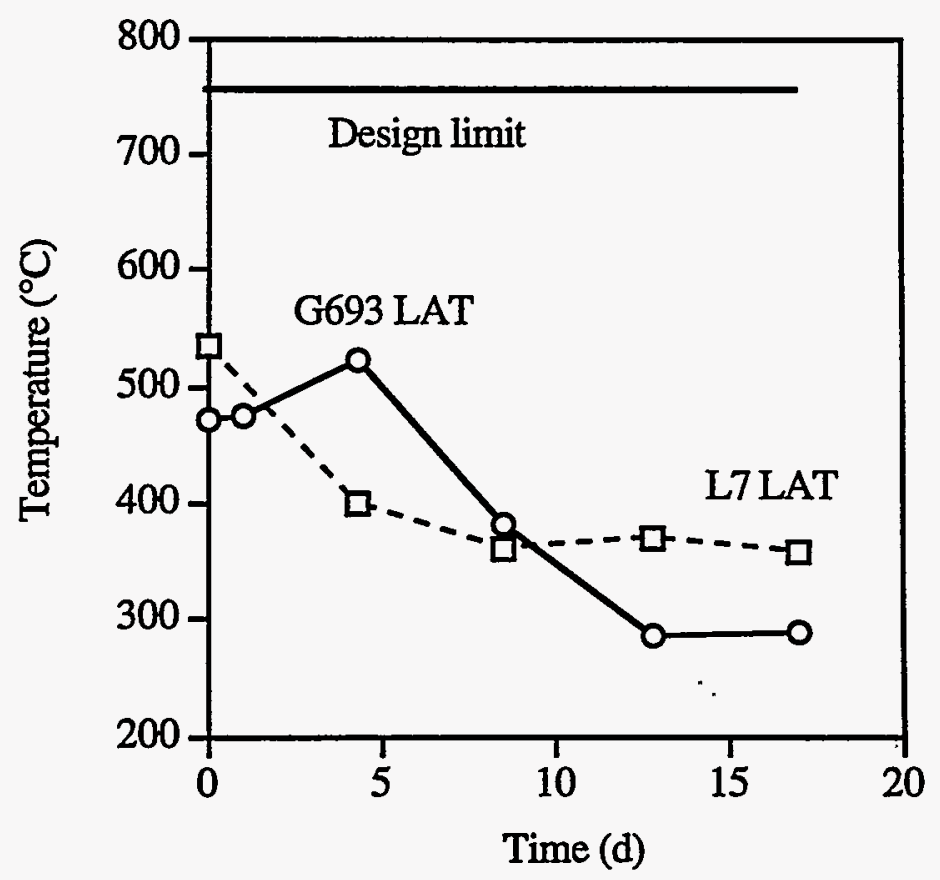

Fig. 6.4. Maximum temperatures from G693 core design LAT analysis compared with the design limit results as a function of time.

Figure 6.5 presents the contours of LAT-produced peaking factor for the first time step for both plates of the G693 core design. Figures 6.6 and 
6.7 show the limited extent of the area of the plate that exceeds $400^{\circ} \mathrm{C}$ maximum segregated fuel temperature. Figure 6.7 presents the temperature results in a carpet plot where the height above the $\mathrm{rz}$ plane is determined by the local maximum temperature. Only a small region near the lower outer corner of the top plate exceeds $400^{\circ} \mathrm{C}$. On the bottom plate, only a thin stripe along the outer edge exceeds this temperature. The rest of the area of the fuel plates is mostly well below this temperature. Figure 6.8 shows the temperature contours on a representation of the plates drawn to scale.

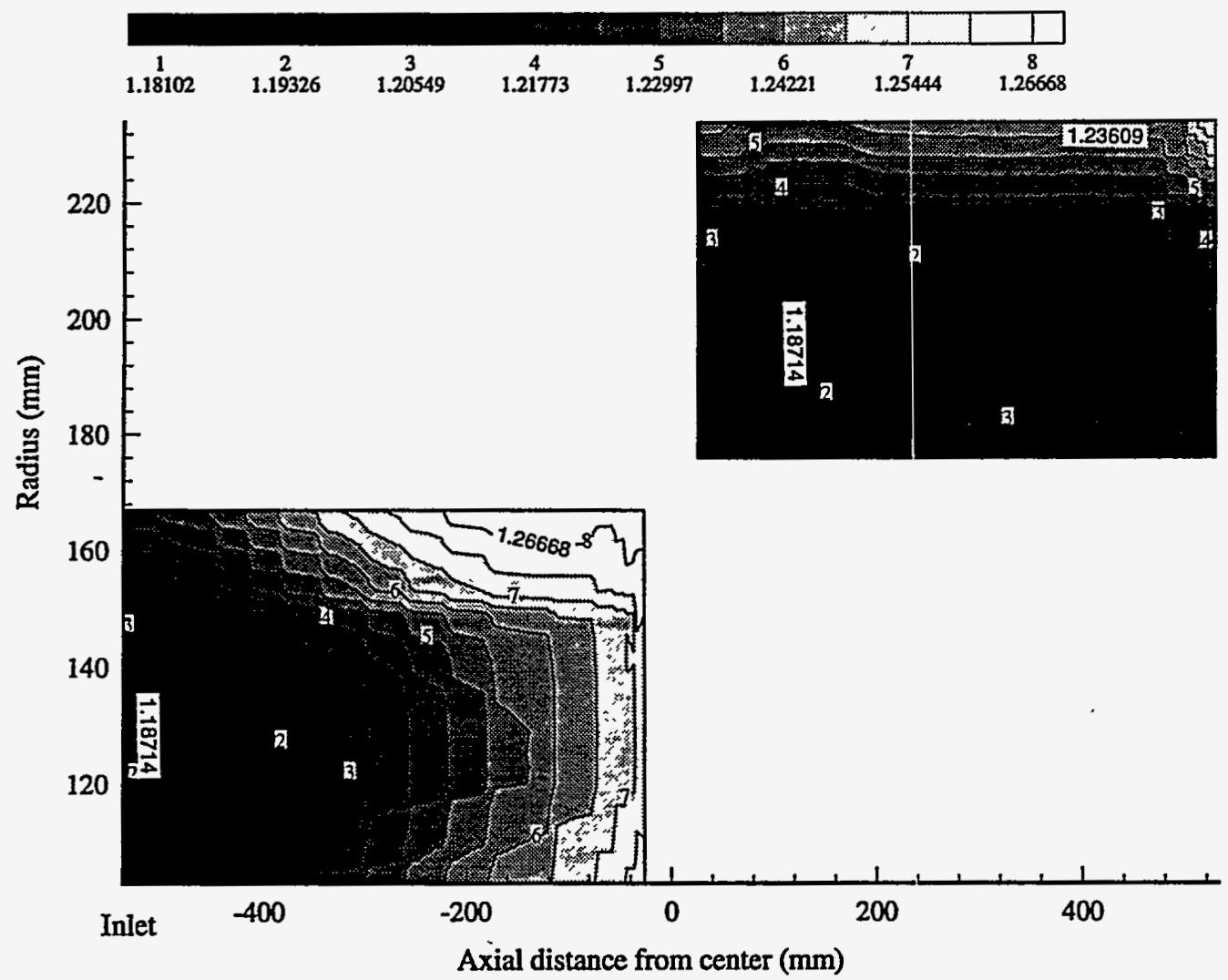

Fig. 6.5. Contours of peaking factor on G693 fuel plates, first time step of LAT analysis.

The high temperatures found in the LAT analyses are generally limited to the segregation volume. Figure 6.9 shows the temperature contours for the axisymmetric hot spot model at the point that produces the highest maximum temperature. The segregation volume is outlined in this figure. The highest thermal gradient is where the temperature contours are closest together. The concentration of contours is almost entirely limited to the segregation region. Figure 6.10 presents the same data on a carpet plot where the vertical coordinate is the local temperature. The surface is quite steep in the segregation volume and relatively level everywhere else. To mark the segregation volume, the grid is overlaid on the temperature surface. 
The contour that is near the segregation volume boundary is labeled to indicate the temperature of this interface, $192^{\circ} \mathrm{C}$. This value is much less than $400^{\circ} \mathrm{C}$. These figures show how limited the region of excessive temperatures is around a segregation volume.

From the differences between the two core designs in the hot spot study results, it was concluded that it should be possible to develop a fuel distribution that could reduce the maximum temperature and peaking factors to acceptable values while satisfying all other constraints. Before this was attempted, the meaning and importance of the thermal limit were examined.

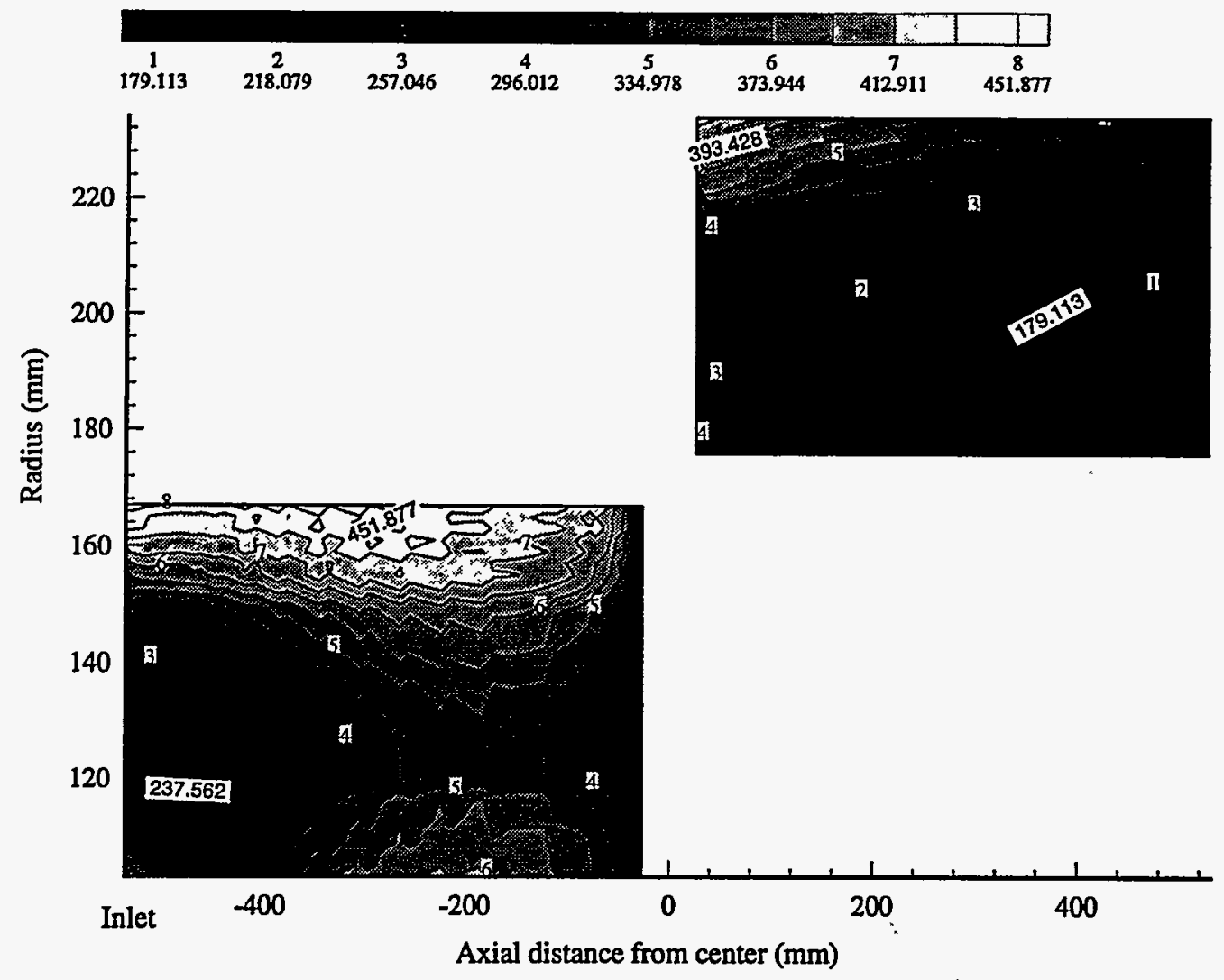

Fig. 6.6. Contours of maximum temperature in segregation volume on G693 fuel plates, first time step of LAT analysis. 


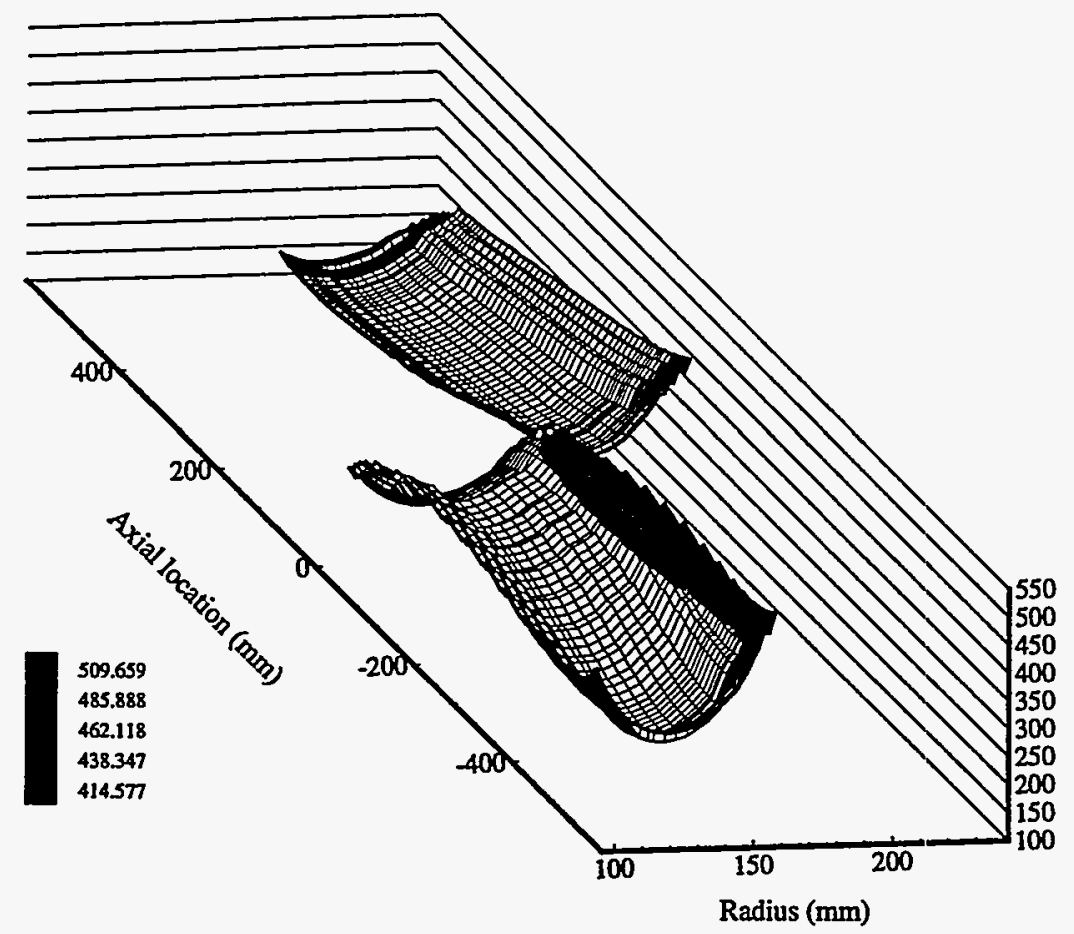

Fig. 6.7. Carpet plot of maximum temperature in segregation volume on G693 fuel plates, first time step of LAT analysis. Shaded region exceeds $400^{\circ} \mathrm{C}$.

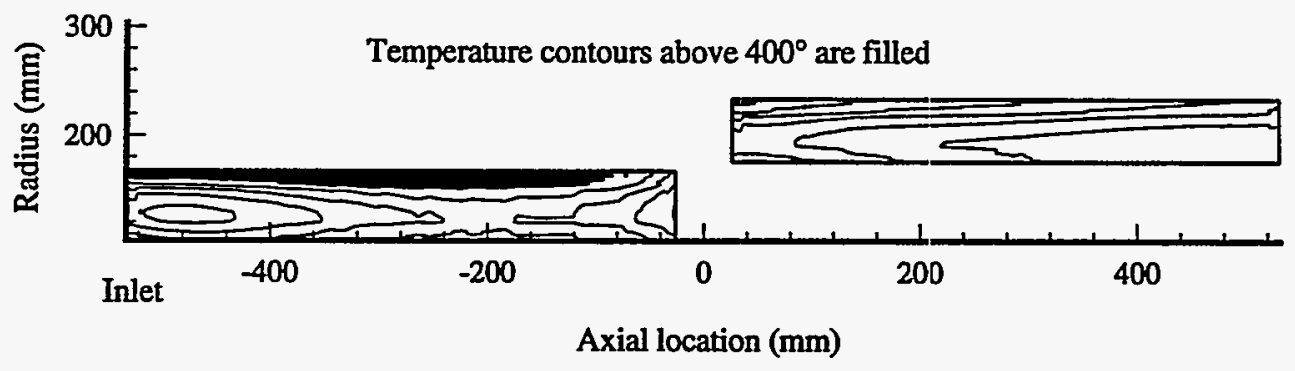

Fig. 6.8. Contour plot of maximum temperature in segregation volume on G693 fuel plates drawn to scale, first time step of LAT analysis. Shaded region exceeds $400^{\circ} \mathrm{C}$. 


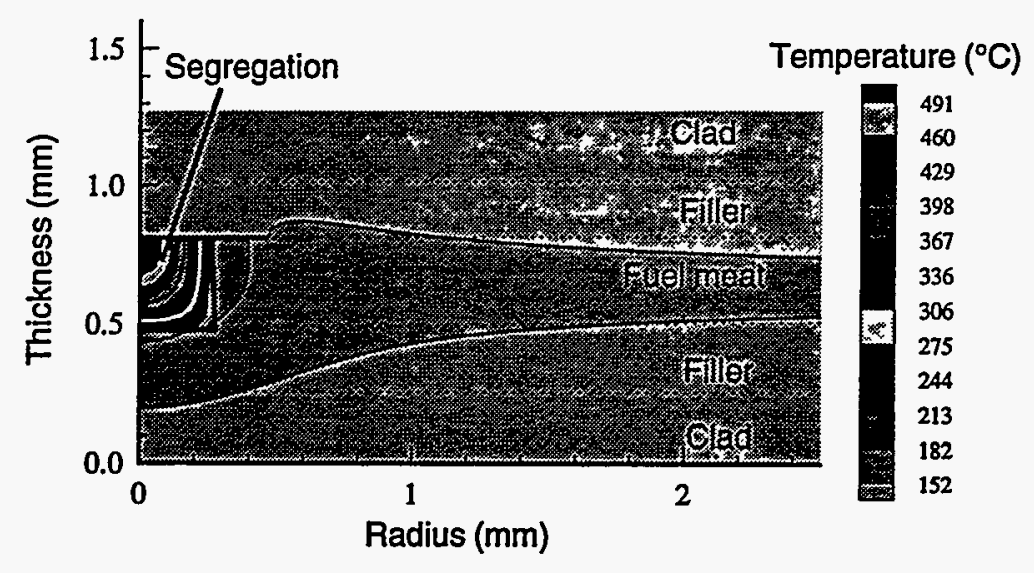

Fig. 6.9. Temperature contours for the highest maximum temperature spot, G693 core design, nominal LAT analysis.

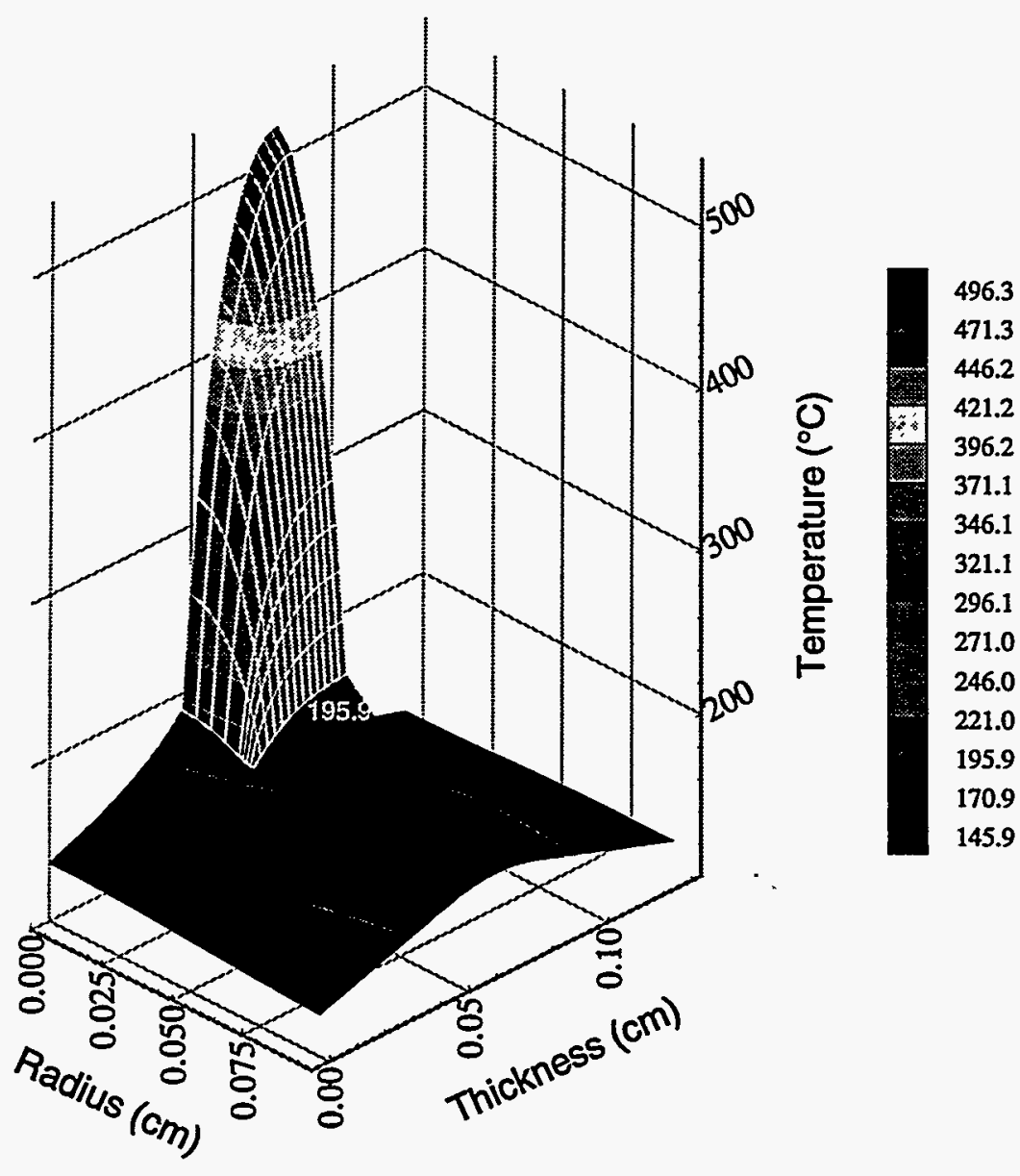

Fig. 6.10. Maximum temperature carpet plot of region near the segregation volume. Region of plot is restricted to $0.1-\mathrm{mm}$ radius. 
The L7 and G693 LAT analyses results indicate that the thermal limit of $400^{\circ} \mathrm{C}$ is still exceeded. What do these results mean? First, it must be remembered that the LAT maximum temperature results are for the temperature within a small volume within the fuel defect pair model. The LAT maximum temperatures are hypothetical results assuming that a nonbond-segregation volume pair that just passes the inspection limits exists at every point. This condition is more severe than possible because of the additional constraints on the inspection process. These defects can be no closer than $2.0 \mathrm{~mm}$, and the volume of the fuel that exceeds the thermal limit is on the order of the segregation volume, $0.2 \mathrm{~mm}$ diam. The inspection process requires that the maximum fuel in a plate is limited; thus there cannot be an unlimited number of segregation volumes. In addition, the numbers of segregation volumes in proximity are limited by the hot streak constraint. This constraint rejects any plate that has $10 \%$ excess fuel in a thin stripe $12.7 \mathrm{~mm}$ long, $0.4 \mathrm{~mm}$ wide. This condition limits the number of segregation volumes in the stripe to 3.88 . Therefore the segregation volumes cannot be closer than $3.2 \mathrm{~mm}$ on average. The minimum VENTURE grid spacing is about $2.0 \mathrm{~mm}$. Thus the maximum temperature results represent local maximum temperatures within very small volumes scattered over the plate. The centerline temperature in normal fuel (maximum local temperature in normal fuel-without a defect) is considerably lower and well below the thermal limit. Figure 6.11 presents the maximum temperature within normal fuel as a function of time for the G693 core design. The results are produced by a 1-D analysis in normal fuel without the presence of defects. This temperature represents the general temperature at each data point and is well below the thermal limit. These results were produced by a 1-D analysis at each data point. Although 2-D conduction between data points was not considered, the results should be very close to the maximum temperatures in the plates at these locations. Comparison with the TASHA code results shows good agreement.

Why is this distinction important? The thermal limit of $400^{\circ} \mathrm{C}$ was set by an examination of experimental plates exposed to fixed temperatures and neutron fluences. The highest temperature that these plates survived without any significant dimensional change was $400^{\circ} \mathrm{C} .{ }^{16}$ Above that temperature, the plates exhibited some swelling. This swelling was assumed to be due to fuel damage. Thus the thermal limit determined for a uniformly heated plate was conservatively assumed for any microscopic volume within the plate. It is highly unlikely that such localized high-temperature regions as calculated by the LAT would be able to affect the overall plate thickness. The result from the LAT and examination of the assumptions behind the ANSR fuel plate thermal limit prompted the "ANSR Fuel Plate Maximum Temperature Workshop" that is discussed in the Sect. 7. 


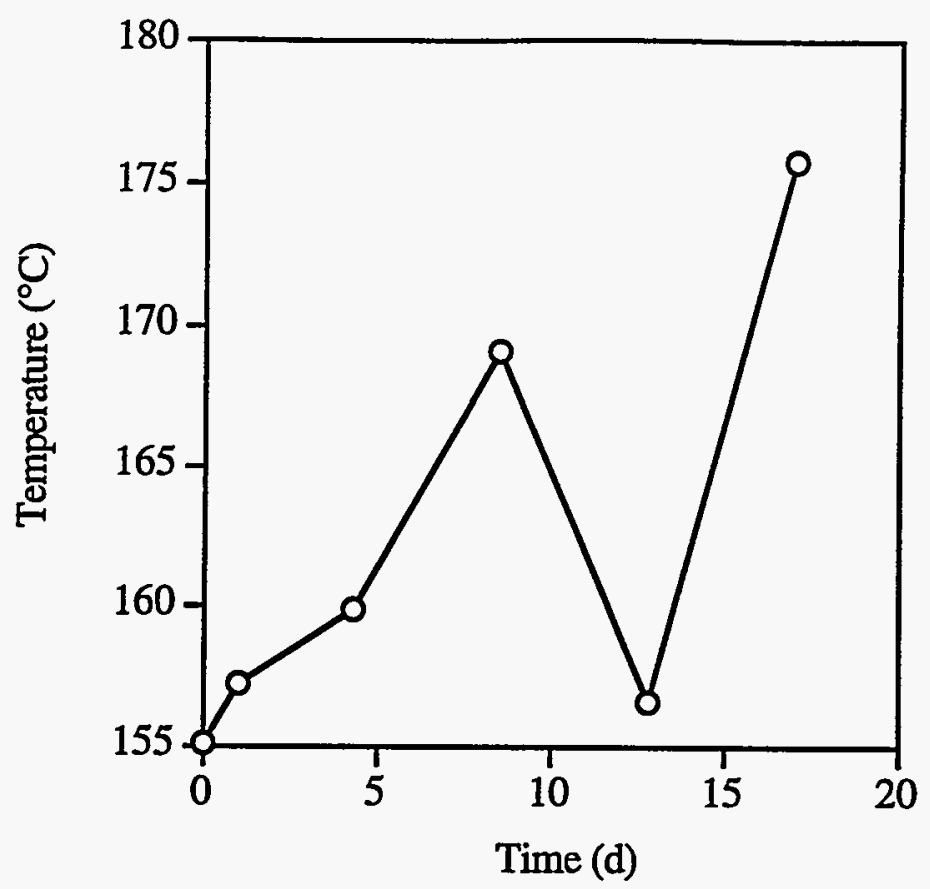

Fig. 6.11. Maximum temperature in normal fuel as a function of time for the G693 core design, LAT results. 


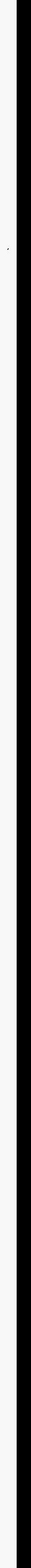




\section{ANSR FUEL TEMPERATURE WORKSHOP}

After review of the L7 and G693 LAT results, it became obvious that extensive efforts would have to be taken in the design optimization in order to meet a $400^{\circ} \mathrm{C}$ maximum fuel temperature limit in the segregation volume. To prevent this effort being expended unnecessarily, the assumptions of the thermal limits were examined in light of current experimental data and fuel property theories. This was accomplished at the "Workshop meeting on ANSR Fuel Plate Temperatures" held at ORNL on January 27, 1994 (see ref.17). The workshop participants included ANSR and HFIR fuels development, analysis and project personnel. The issue of fuel temperature was discussed from all perspectives.

The main motivation for the workshop stemmed from the high temperatures being calculated in the hot spot studies. In the studies, the excessive temperatures were limited to a volume about the size of the segregation volume. These temperatures occurred in a cylinder $0.3 \mathrm{~mm}$ in diameter and $0.2 \mathrm{~mm}$ long. The temperatures exterior to this cylinder were typically only slightly elevated above the temperature expected in normal fuel without defects. The temperature of this normal fuel mixture was $200^{\circ} \mathrm{C}$ or less.

It was the unanimous conclusion of the fuel development personnel that this small, high-temperature region would not be likely to cause a problem in the plate. The $400^{\circ} \mathrm{C}$ thermal limit is still appropriate for the average temperature of fuel plate volumes larger than the segregation volume. The minimum size of this region was not determined but was thought to be several times the size of the segregation volume. Thus the excessive temperatures found in the LAT analyses were not harmful and would not require an optimization effort specifically intended to minimize the temperatures in the segregated material. The maximum temperature in the normal fuel would still be the effective acceptance criterion.

Also discussed at the workshop were the latest experimental data on irradiated $\mathrm{U}_{3} \mathrm{Si}_{2}$ fuel. These data indicate that irradiated $\mathrm{U}_{3} \mathrm{Si}_{2}$ dispersed in aluminum at high temperatures is converted into $\mathrm{U}[\mathrm{Al}(75), \mathrm{Si}(25)]_{3}$ by aluminum that diffuses into the particles. This compound has the $\mathrm{UAl}_{3}$ crystal structure and apparently its good retention of fission gases and resistance to bubble formation. This conversion significantly reduces the thermal conductivity. The conversion is considered to occur above $400^{\circ} \mathrm{C}$.

\subsection{G693 ANALYSIS - CONVERSION OF FUEL TO U(AL,SI) 3 MODEL}

The conversion of the fuel particles under irradiation at high temperatures produces a complex thermal behavior that was incorporated 
into the G693 LAT analysis. The conversion would reduce the thermal conductivity at the center of the segregation volume and therefore it would increase the maximum temperature estimates. The rate and completeness of this conversion are not known as functions of temperature. It was therefore conservatively assumed that the conversion was complete and instantaneous for any of the fuel reaches $400^{\circ} \mathrm{C}$. This assumption was applied to both the segregated and normal fuel materials.

It was assumed that the aluminum at these high temperatures is mobile enough to migrate from the segregated material and convert all the material that exceeded the conversion temperature. This migration was assumed to remove all of the aluminum from the segregated noaterial. The swelling of the fuel particles is assumed to close all of the voids. Therefore, this segregated material outside of the central region was conservatively assumed to be converted to pure fuel. These assumptions produce a region of pure $\mathrm{U}(\mathrm{Al}, \mathrm{Si})_{3}$ within a cylinder of pure $\mathrm{U}_{3} \mathrm{Si}_{2}$. This is modeled in the LAT as a cylinder of $\mathrm{U}(\mathrm{Al}, \mathrm{Si})_{3}$ within a cylinder of $\mathrm{U}_{3} \mathrm{Si}_{2}$.

The DART model was modified to incorporate information from new irradiation experiments. These modifications produced estimates of conductivities that exhibited more complex behaviors. ${ }^{18}$ Figure 7.1 presents the new conductivity estimates for normal and segregated fuel as a function of fission density. Figure 7.2 shows the behavior: of the pure $\mathrm{U}_{3} \mathrm{Si}_{2}$ and $\mathrm{U}(\mathrm{Al}, \mathrm{Si})_{3}$ materials as a function of fission density. These estimates were used in analyses of the G693 core design. In these analyses the conductivity of the pure $\mathrm{U}_{3} \mathrm{Si}_{2}$ was assumed to be a constant whenever temperatures were above the maximum reported from the DART code. The DART calculations were halted when conditions exceed the applicability of the assumptions.

The volume changes due to the chemical changes and the closure of the voids are assumed to balance out. The model assumed that there is no net change in the size of the segregation region.

The uranium in the segregation volume is assumed to remain in place during this conversion process. Only the aluminum is assumed to be mobile. Thus, the heat generation rate per unit volume in the new composite $\mathrm{U}(\mathrm{Al}, \mathrm{Si})_{3} / \mathrm{U}_{3} \mathrm{Si}_{2}$ segregation volume is assumed to be the same as in the initial segregated $50 \% \mathrm{U}_{3} \mathrm{Si}_{2}$ material.

The maximum temperatures from two cases are shown in Fig. 7.3. Both cases use the new model for the segregation volume with conversion to $\mathrm{U}(\mathrm{Al}, \mathrm{Si})_{3}$. The case labeled $95 \%$ used the $95 \%$ probability values and the nominal case used the nominal values as described in Sect. 4.2. The temperatures are much higher $\left(1100^{\circ} \mathrm{C}\right)$ than the previous thermal models, but they are lower than the melting temperature of either the $\mathrm{U}(\mathrm{Al}, \mathrm{Si})_{3}$ or $\mathrm{U}_{3} \mathrm{Si}_{2}$ material by at least $250^{\circ} \mathrm{C}$. The melting point of $\mathrm{UAl}_{3}$ is $1350^{\circ} \mathrm{C}$. The 
$\mathrm{U}(\mathrm{Al}, \mathrm{Si})_{3}$ material should have a melting point on the same order. The melting point of the $\mathrm{U}_{3} \mathrm{Si}_{2}$ is $1665^{\circ} \mathrm{C}$.

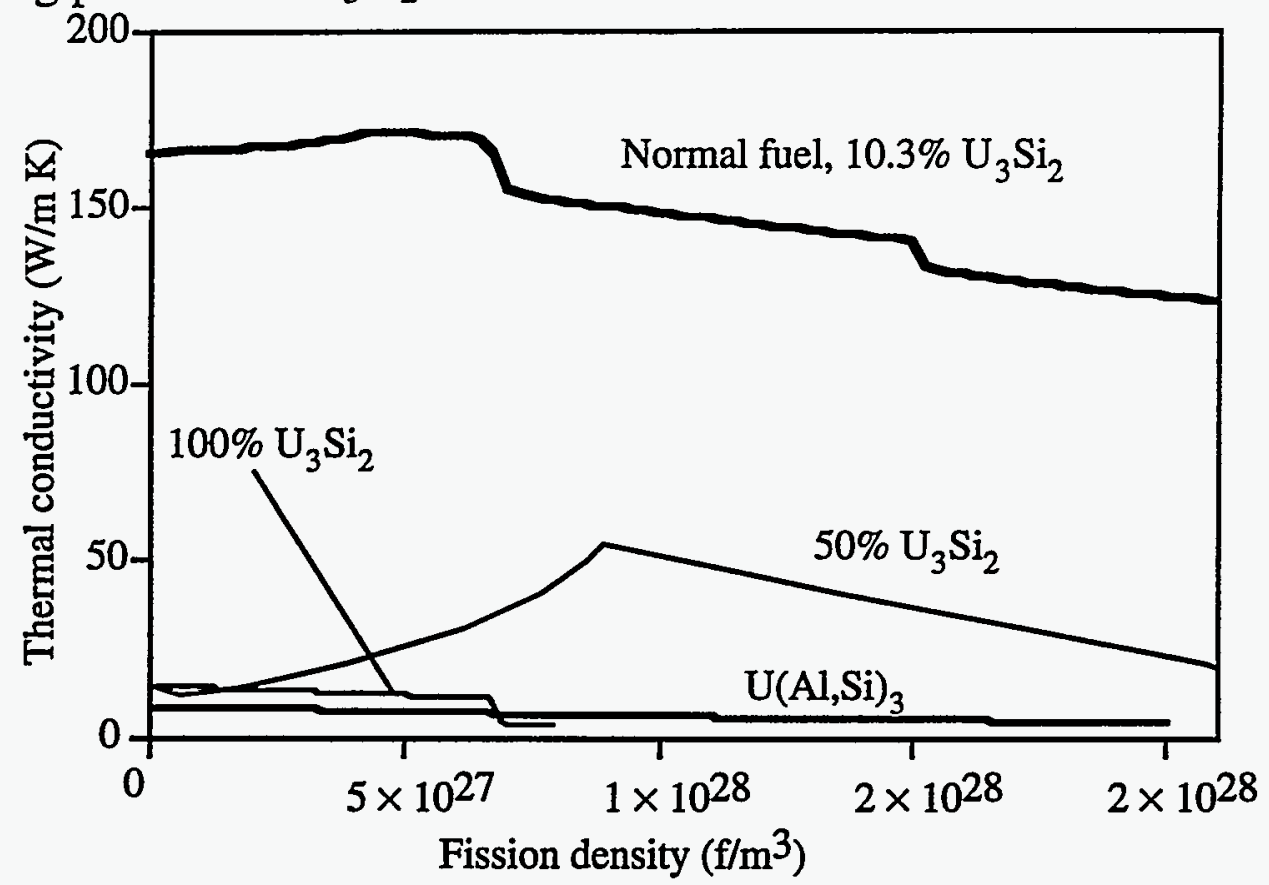

Fig. 7.1. DART thermal conductivity estimates for HEU fuel.

Since the DART conductivity estimates are homogenized effective conductivities, it is possible that the temperature within the center of the fuel particle could be high enough to produce localized melting. It is not clear that localized melting within the fuel particles is a dangerous condition. It is possible that some experimental samples have undergone localized melting at the center of a particle. No indications of problems due to localized melting have been found.

The maximum temperature in the normal fuel adjacent to the segregation volume is considerably lower than in the segregation volume, as shown in Fig. 7.4. This figure presents a carpet plot of the temperature around the hot spot that produces the highest temperature on either plate. The region that contains the $\mathrm{U}(\mathrm{Al}, \mathrm{Si})_{3}$ is marked by the grid overlay on the temperature surface. The maximum $\left(347.3^{\circ} \mathrm{C}\right)$ and minimum $\left(136^{\circ} \mathrm{C}\right)$ contours that exist in the $\mathrm{U}_{3} \mathrm{Si}_{2}$ material that makes up the remainder of the segregation volume are also marked. Although the temperatures in the center of the composite segregation cylinder are quite high, these excessive temperatures are extremely limited in volume. The temperature of the normal fuel is well below the thermal limit. The lower conductivity of the $\mathrm{U}_{3} \mathrm{Si}_{2}$ material limits the temperature in the normal fuel and exacerbates the high temperatures. 


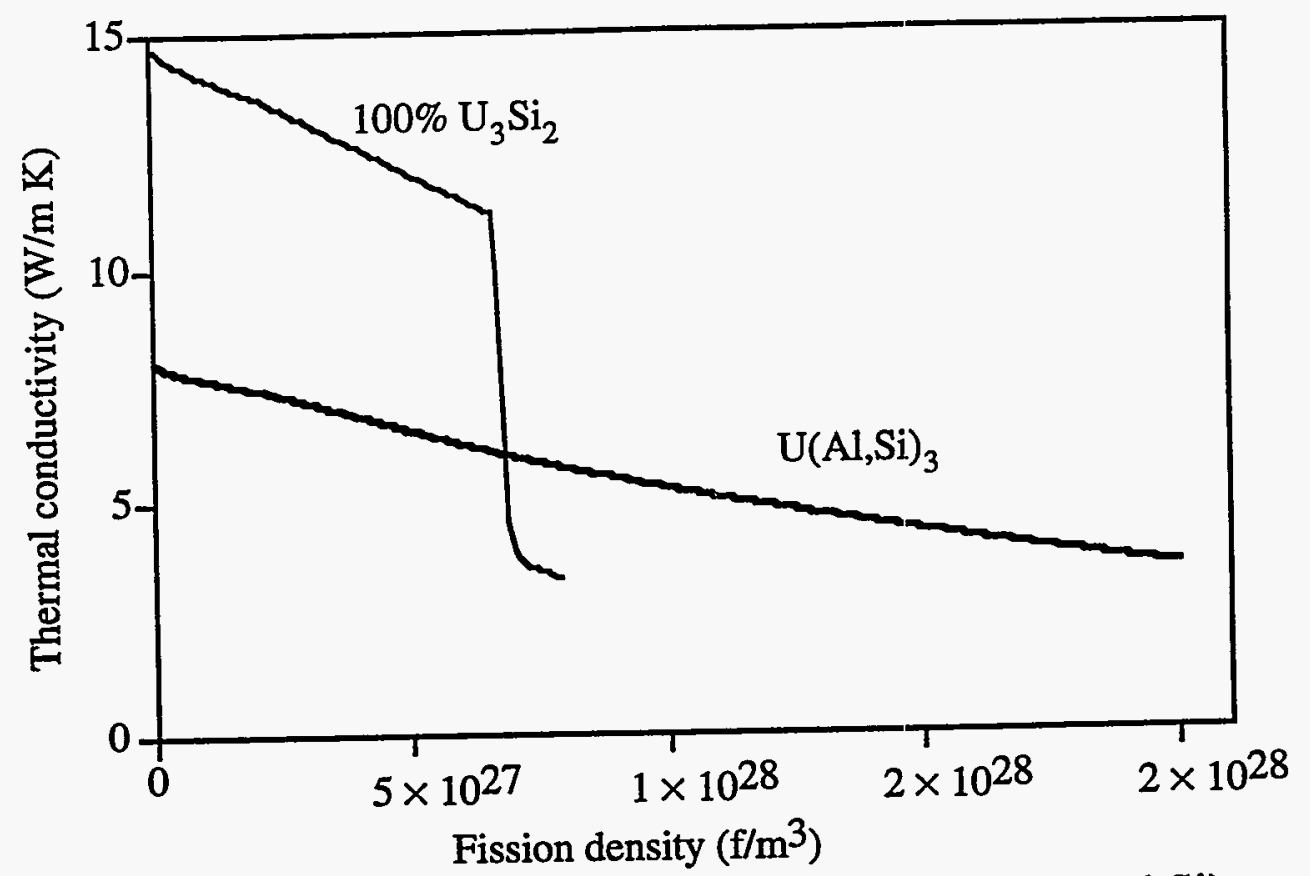

Fig. 7.2. Thermal conductivity for pure $\mathrm{U}_{3} \mathrm{Si}_{2}$ and $\mathrm{U}(\mathrm{Al}, \mathrm{Si})_{3}$.

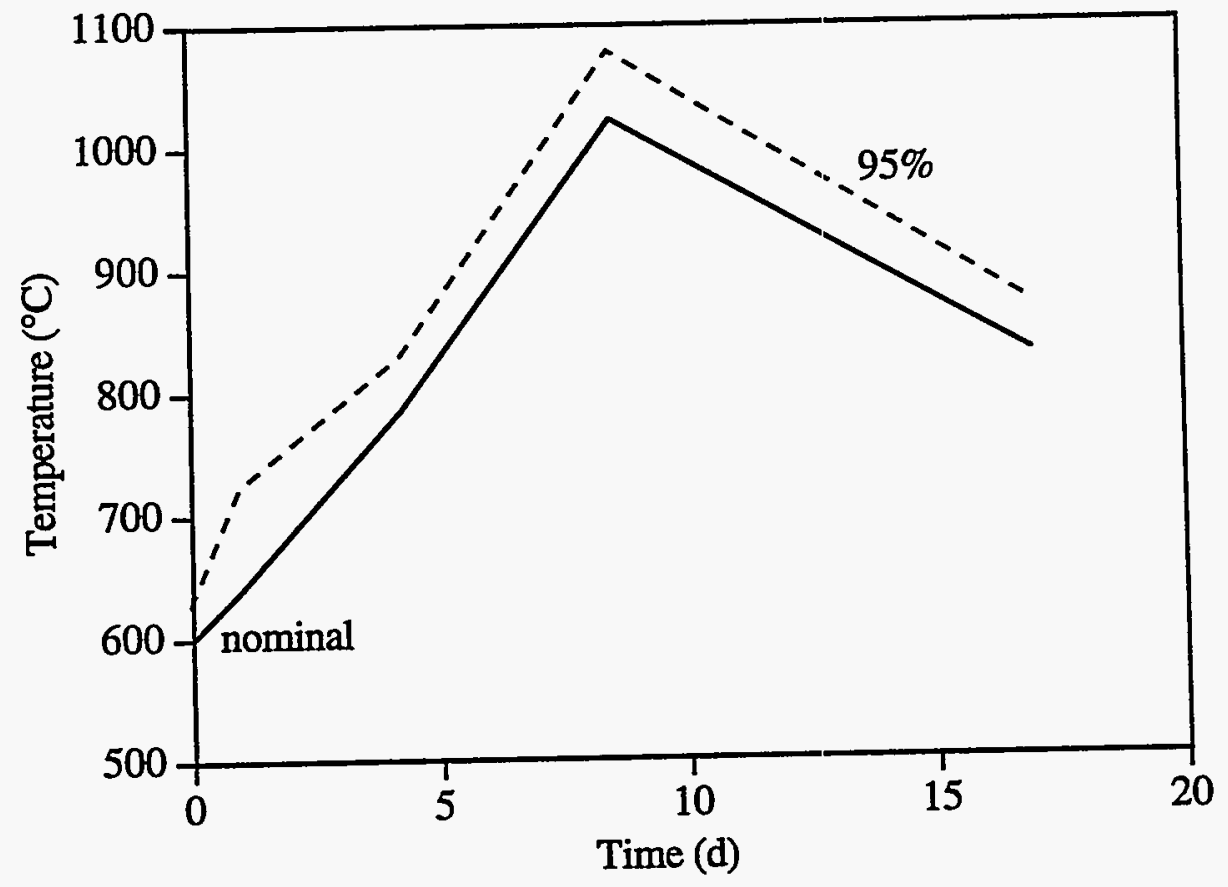

Fig. 7.3. Maximum temperatures in a G693 LAT analysis with conversion of fuel to $\mathrm{U}(\mathrm{Al}, \mathrm{Si})_{3}$. 


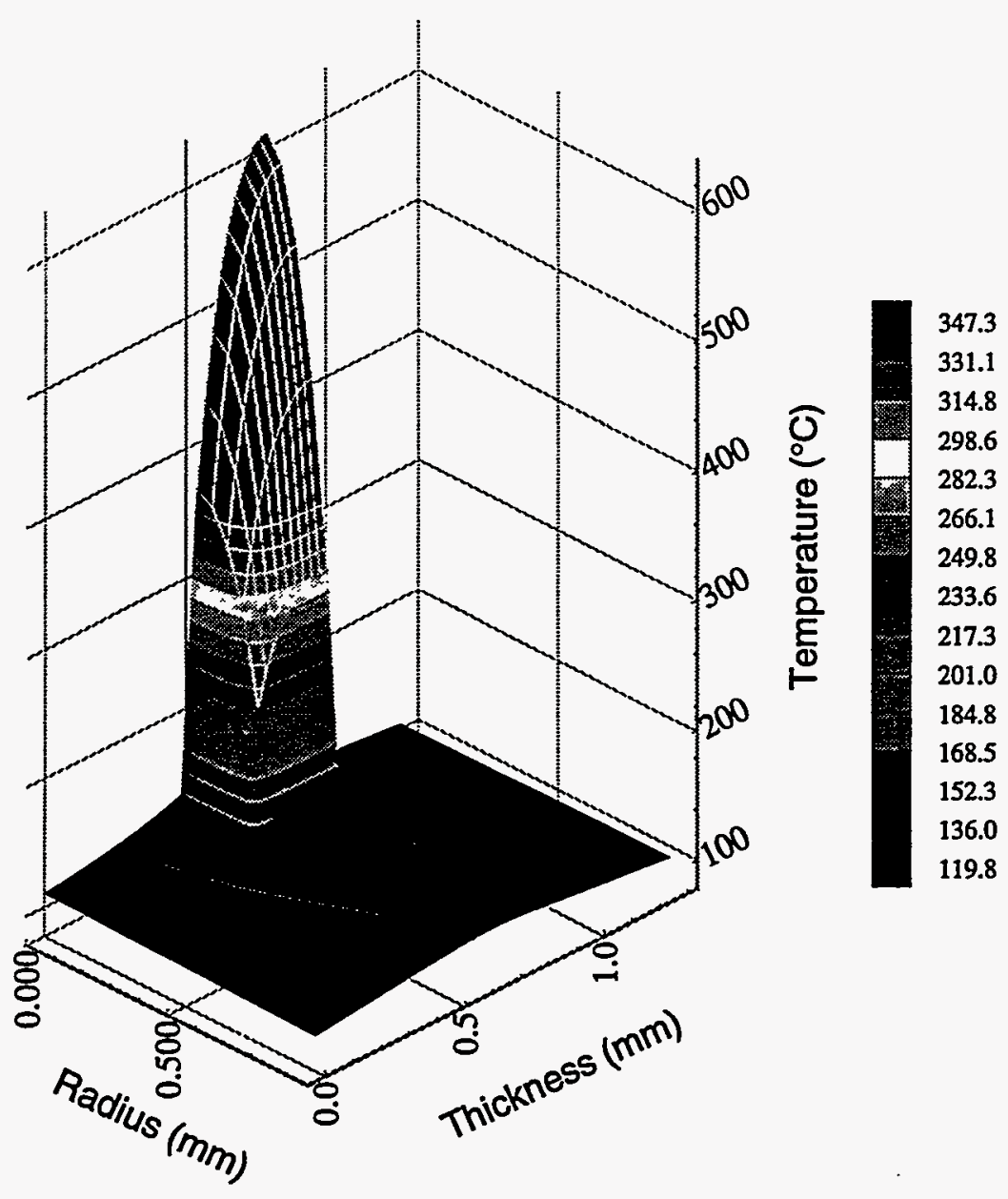

Fig. 7.4. Carpet plot of temperatures around hot spot with highest temperature. G693 LAT results with fuel conversion to $\mathrm{U}(\mathrm{Al}, \mathrm{Si})_{3}$.

These results were generated with a model that assumes that the conversion of $\mathrm{U}_{3} \mathrm{Si}_{2}$ to $\mathrm{U}(\mathrm{Al}, \mathrm{Si})_{3}$ occurs at a temperature of $400^{\circ} \mathrm{C}$. This transition temperature is not known precisely. The conversion will likely occur over a range of temperatures, with an increasing rate and an increasing completeness of the conversion with temperature. The potential effect of this assumed transition temperature was examined using this model by modifying the transition temperature over a range of 350 to $450^{\circ} \mathrm{C}$. Figure 7.5 presents the maximum temperature results from these analyses. The lower transition temperature increases the temperatures both early and late in the transient. However, the largest maximum temperature is about the same for all three transition temperatures. The peaking factor is not affected significantly by the transition temperature. 


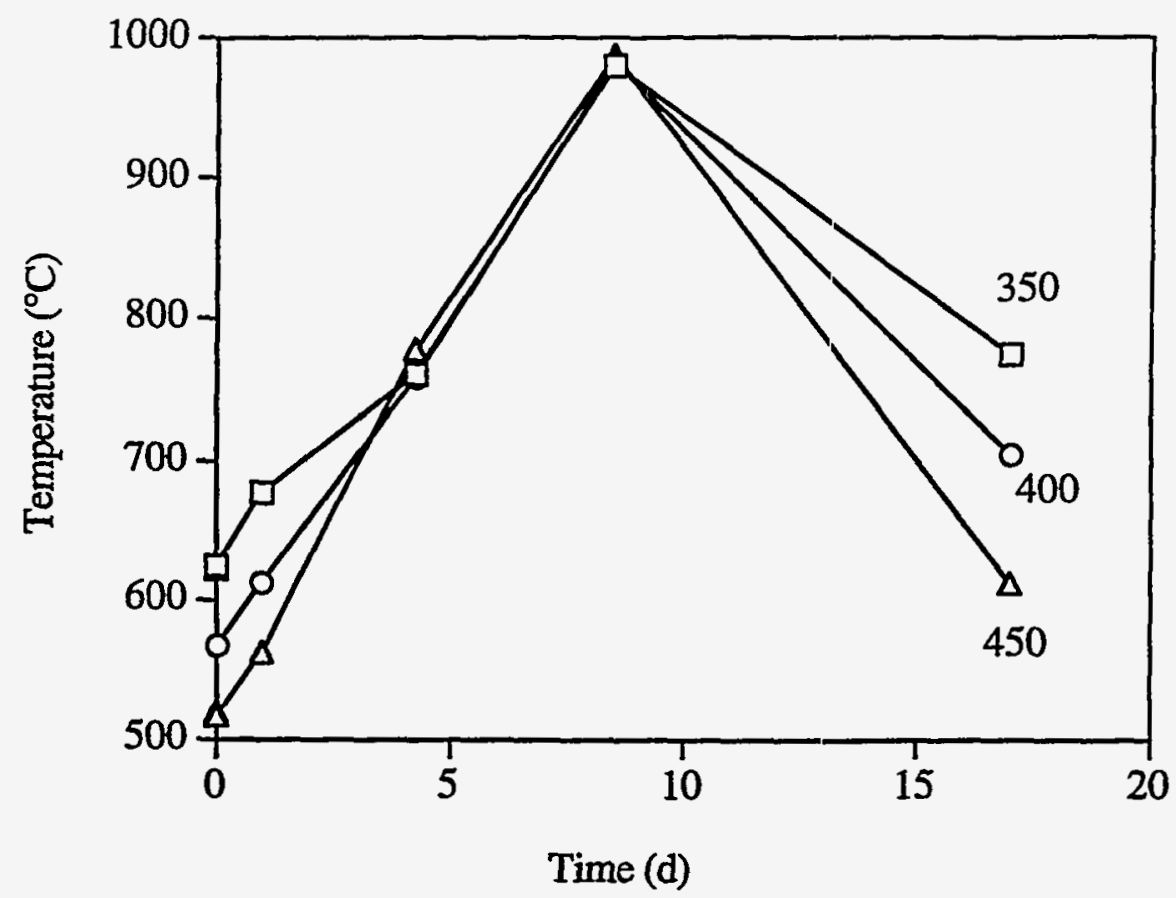

Fig. 7.5. Maximum temperatures from G693 LAT analysis using different transition temperatures.

The current model incorporating these complex phenomena was used to analyze various inspection procedures and other phenomena. Reducing the inspection spot size on the excess fuel loading limit reduces the maximum temperatures and peaking factors, as shown in Figs. 7.6 and 7.7. Reducing the amount of excess fuel loading allowed also reduces these parameters. These cases used segregation inspection spot sizes of $1-\mathrm{mm}$ diam and the standard 2-mm diam. The fuel overload factors (FOs) used were the standard of $20 \%$ and a reduced value of $10 \%$.

The effect of the assumed transition temperature is more significant than shown in Fig. 7.5 for some of these cases. For example, Fig. 7.8 shows the maximum temperatures for a case using the standard inspection spot size and a reduced $\mathrm{FO}$ of $10 \%$, the nonlinearity of the transition becomes evident. The case with the $400^{\circ} \mathrm{C}$ transition temperature did not undergo significant transition and the temperature remained below $400^{\circ} \mathrm{C}$. The case using a $350^{\circ}$ assumed transition temperature did transition, and the resulting temperatures were much higher. 


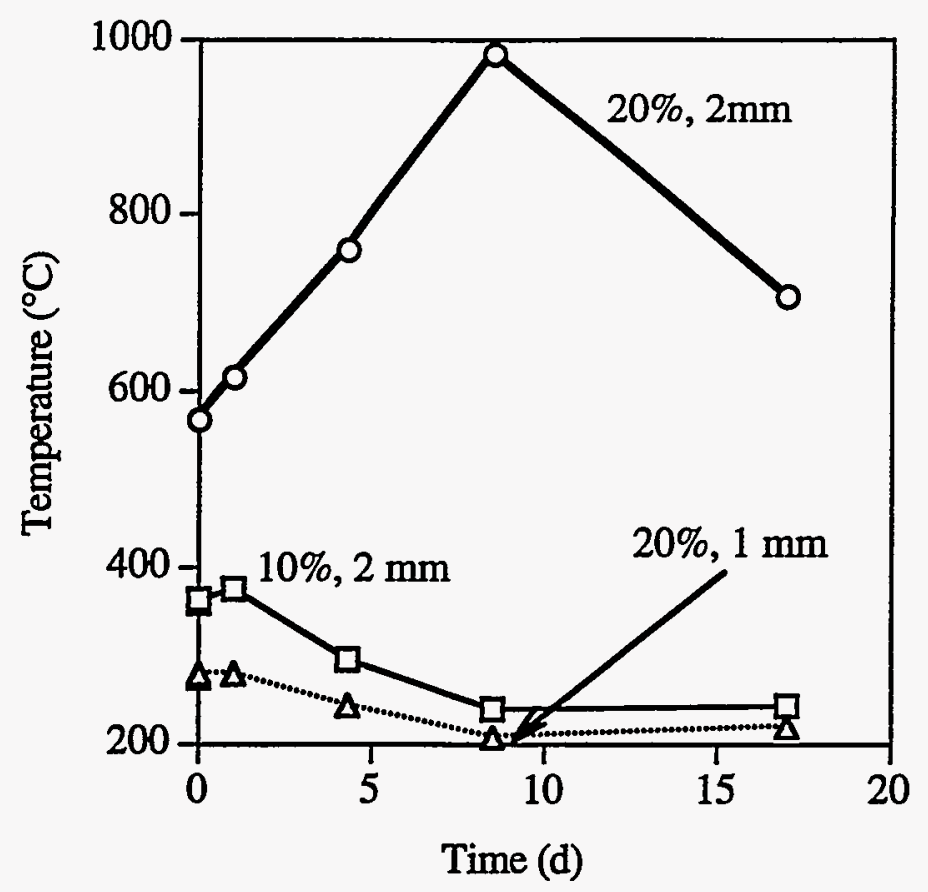

Fig. 7.6. Maximum temperature for G693 LAT analyses using standard 2-mm and 1-mm-diam inspection spot size, $20 \%$ and $10 \%$ FOL.

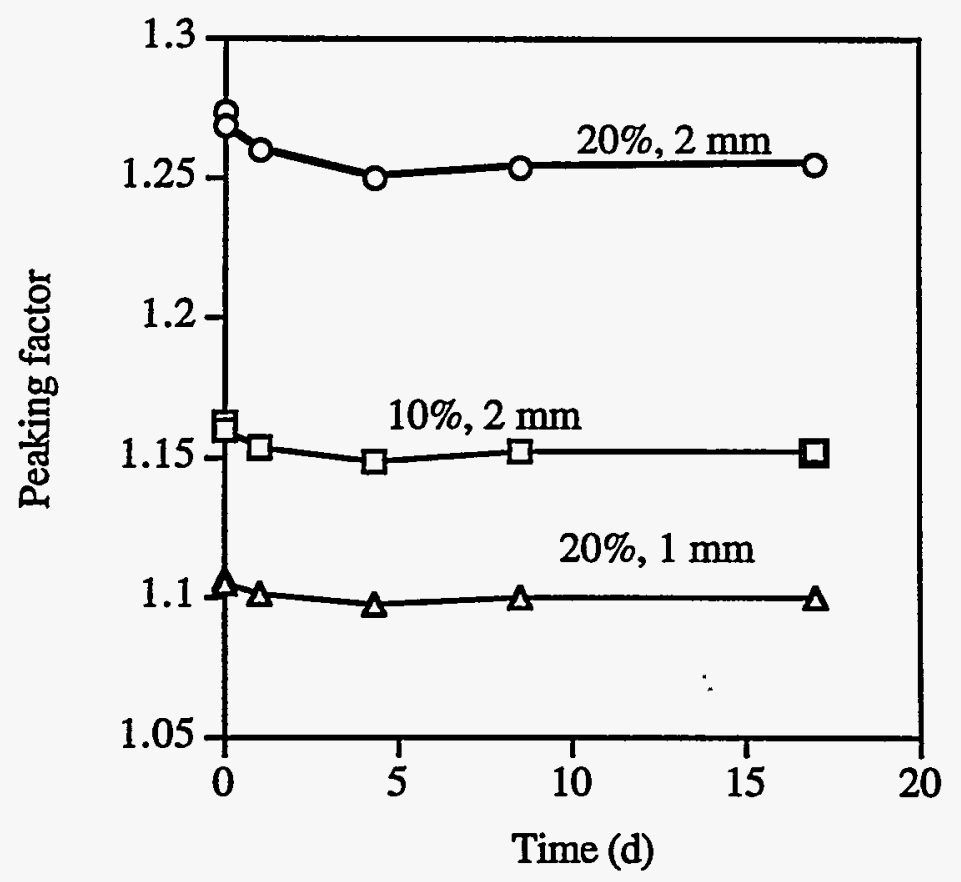

Fig. 7.7. Maximum peaking factors for $\mathrm{G} 693$ LAT analyses using standard $2-\mathrm{mm}$ and 1-mm-diam inspection spot size, $20 \%$ and $10 \%$ FOL. 


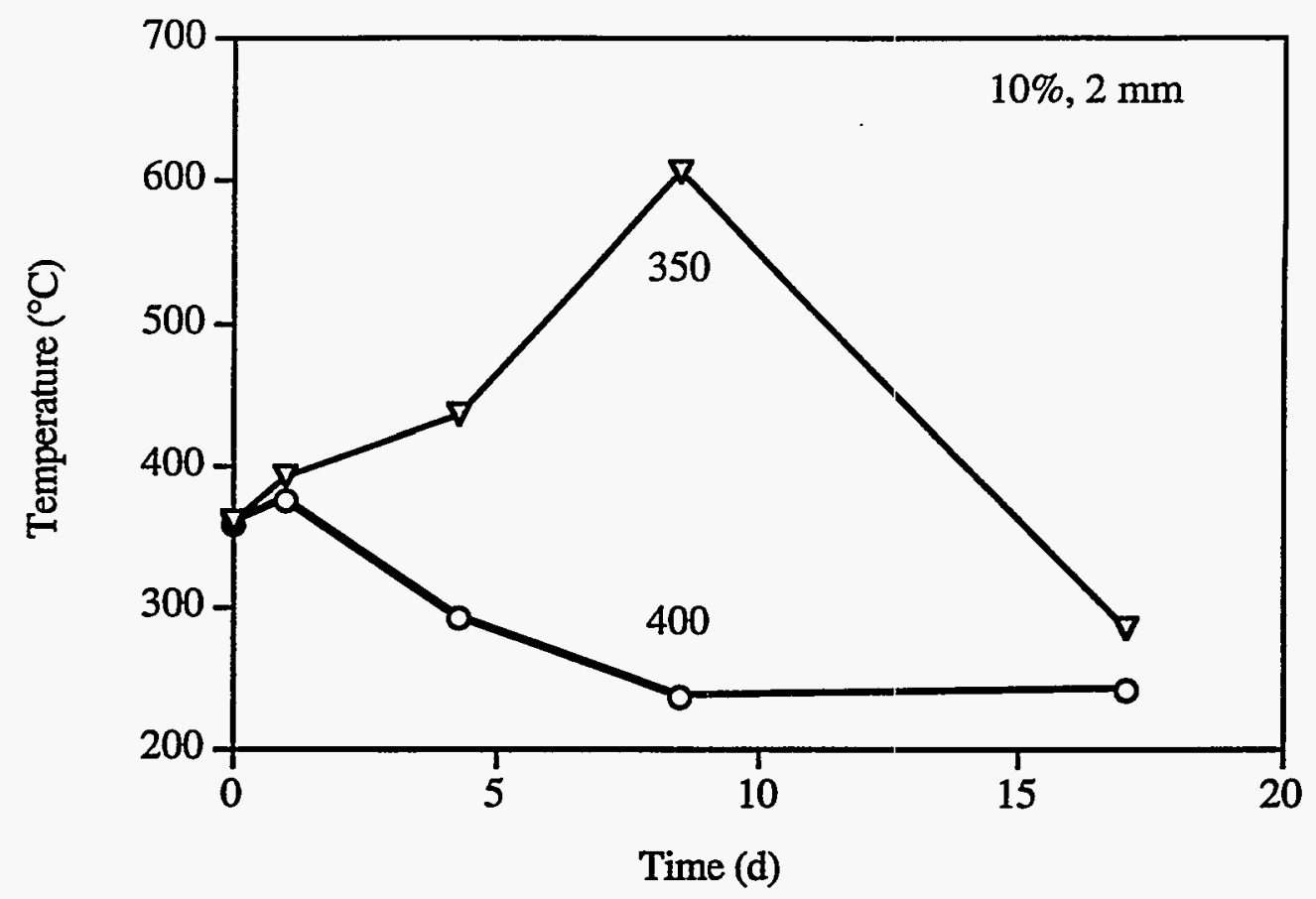

Fig. 7.8. Maximum temperature for G693 LAT analyses using standard 2-mm-diam inspection spot size, $10 \%$ FO with different transition temperatures.

\subsection{NON-ADIABATIC NONBOND}

Most previous models used for the hot spot analysis assumed that the nonbond was an adiabatic volume. No heat was transferred across this region. It is likely that some gas will exist in the nonbond cavity and that the nonbond will be of a significant thickness. As the fuel cycle progresses, some gaseous fission products could escape into the nonbond, changing the composition of the gas in the nonbond cavity. This could produce a pressure rise that could increase the thickness of the nonbond volume. If a large enough pressure is applied it is possible to propagate the nonbond void further. G. T. Yahr ${ }^{19}$ produced an analysis that estimates the pressure needed to propagate the nonbond as a crack in the material at 7.6 MPa.

The thermal model used in the LAT was modified to include the effects of gas in the nonbond void and thermal radiation across the nonbond void. The gas for this analysis was assumed to be air at atmospheric pressure. Air was chosen because it is a likely contaminant in the fuel plate manufacture and the conductivity of air is higher than most of the candidate gases (krypton, argon, xenon). Although gases like helium and neon have higher thermal conductivities than air they are not expected to be present. The nonbond cavity analyzed was a disk of uniform thickness of 0.1 or 
$0.34 \mu \mathrm{m}$. Yahr indicated that $0.34 \mu \mathrm{m}$ is the maximum thickness of the nonbond cavity at $7.6 \mathrm{MPa}$. The conductivity of air does not change significantly with increasing pressure so normal pressure conductivity was used in the model. The cavity is small enough that convective effects can be neglected.

The only potential modification to the conductivity that could be identified was the temperature-jump condition that occurs when the gap thickness is on the order of one-tenth the mean-free-path. The mean-free-path of air molecules at $1 \mathrm{~atm}$ is about $6.0 \times 10^{-8} \mathrm{~m}$ or $0.06 \mu \mathrm{m}$. This condition would occur for only a small portion of the maximum thickness nonbond and would reduce the conductivity by 10 to $20 \%$. This small effect was not included in the results that follow.

Figure 7.9 presents the maximum temperature for a model that incorporates the thermal conductivity of air in the nonbond cavity of two thicknesses $(0.1$ and $0.34 \mu \mathrm{m})$. This analysis is for the G693 core design with nominal values. The maximum temperatures from a model without a nonbond (perfect thermal contact across the meat filler interface) are also included for comparison. Air in the nonbond cavity can produce a $200^{\circ} \mathrm{C}$ to $300^{\circ} \mathrm{C}$ drop in the maximum temperature. The gas in the nonbond void would likely include lower conductivity gases later in the cycle. Thus the actual temperature reduction is expected to be less than calculated here.

Included in this model was an approximation of the thermal radiation across the nonbond cavity. This model assumed that both surfaces were black (perfect absorbers-emitters). The magnitude of the effect of this heat transfer mechanism is not large $\left(-5^{\circ} \mathrm{C}\right)$. In reality the surfaces would not be totally black and the heat transferred by radiation would be less than in this model.

For the initial nonbond there may be significant thermal contact in addition to the gas conduction and thermal radiation. This effect will be reduced quickly as the surfaces move apart under the influence of the internal pressures. These contacts were not included in this model since the maximum temperatures occur later in the cycle after the internal pressures have been assumed to increase significantly. If the postulated pressure increase does not happen then the effective conductivity of the nonbond cavity could be much larger than in this model but not as large as perfect thermal contact. 


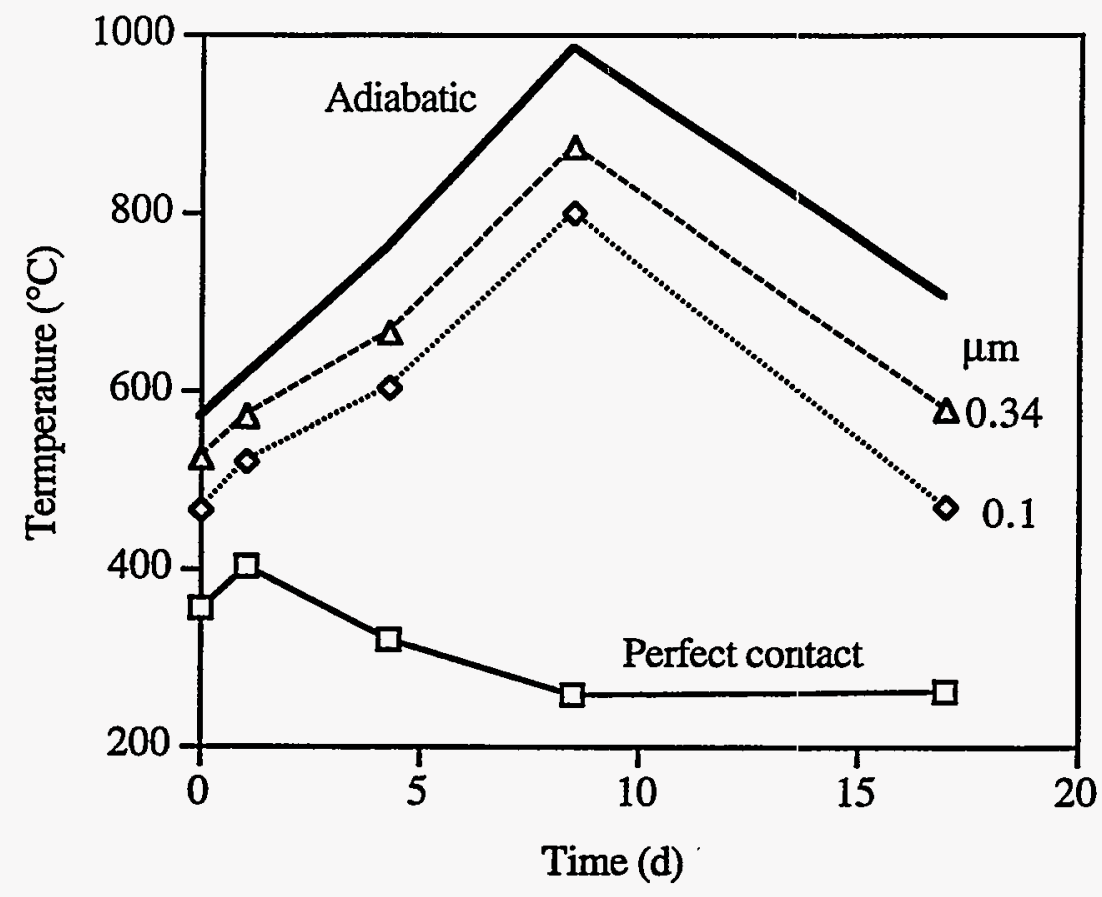

Fig. 7.9. HEATING maximum temperatures for the G693, 300-MW, "best-estimate" case. Comparison of adiabatic and perfect thermal contact models with models using air in nonbond cavity.

Yahr's calculations indicate that the nonbond cavity would have a thickness variation across the radius, with the maximum at the centerline and zero at the edges. I investigated this thickness variation by approximating the cavity as a series of concentric disks of different thicknesses. This model produced maximum temperatures that were within $20^{\circ} \mathrm{C}$ of the uniform thickness cases. 


\section{ENRICHMENT STUDIES}

In late 1994 the ANSR design was modified to use $50 \%$ or $35 \%$ enriched fuel with a much higher fuel density of $3.5 \mathrm{~g} / \mathrm{l}$. These changes modified the thermal behavior of the normal and segregated fuels. Figures 8.1 and 8.2 present the new . conductivity estimates for normal and segregated fuel. These figures are plotted as a function of the burnup fraction for comparison. Since the enrichment levels are quite different between these two materials, the maximum fission densities in the fuel particles are different. Note in Fig. 8.1 that the conductivity of the 50\%- and $35 \%$-enriched, 3.5-g/l normal fuel is much lower than the $93 \%$-enriched, 1.3-g/l density fuel used in the earlier core designs. Estimated thermal conductivity for the $35 \%$-enriched segregated material was not produced.

The modified fuel specifications and other design considerations required a new core design. However, the calculations reported in this section were performed on a modified G693 core design because the new design was not complete at the time these calculations were requested. The continued development of the LAT technique and extension to the newer core designs was not completed. The LAT was used with this new fuel material to investigate several issues that relate to the inspection specifications. These investigations are reported in this section.

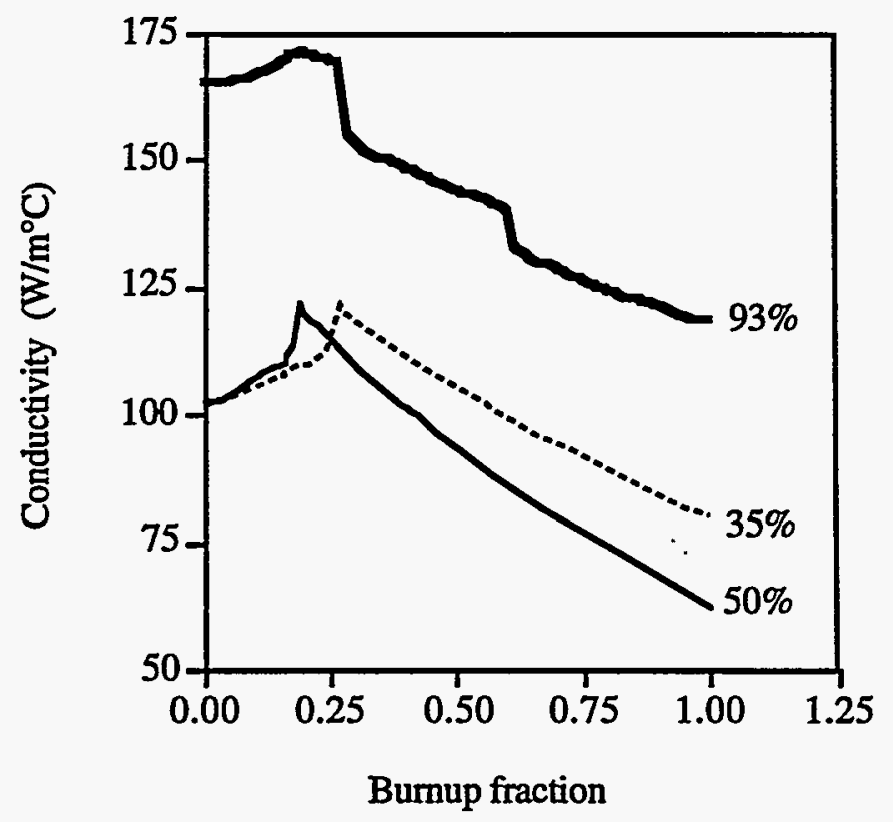

Fig. 8.1. Thermal conductivity estimates for high-enriched and medium-enriched normal fuel. 


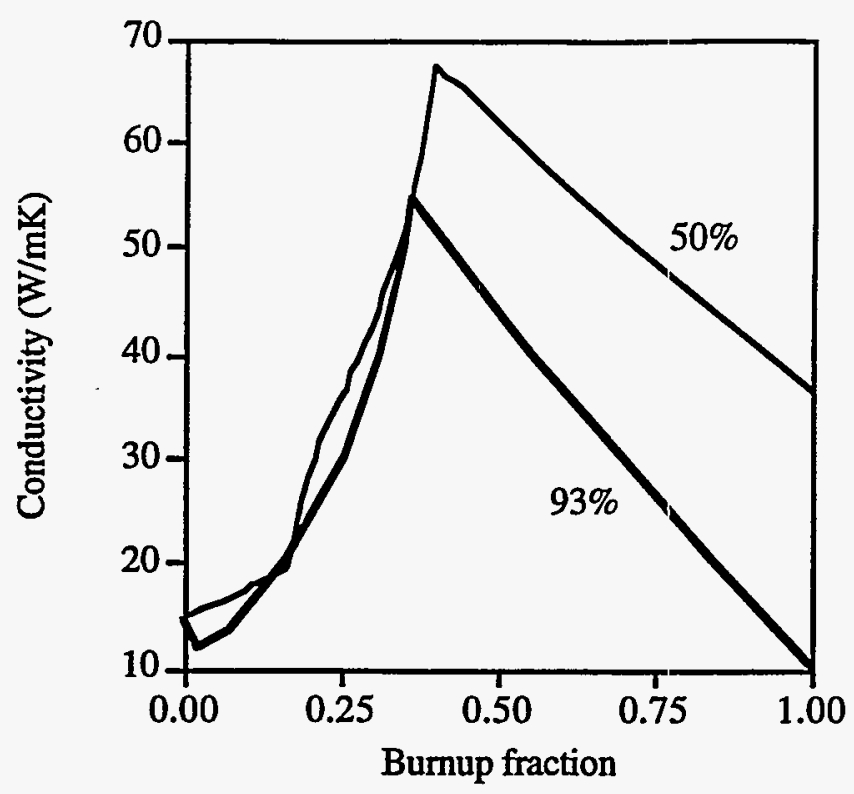

Fig. 8.2. Thermal conductivity estimates for high-enriched and medium-enriched segregated fuel.

The effect of changing the fuel density and enrichment was investigated by using the G693 core design as a means of varying the thermal parameters and fission density is a reasonable manner. The fission density of the G693 design was converted to burnup fraction as a function of time. This variable was used to interpolate the local thermal conductivities and these were used in a LAT analysis of the G693 core. This analysis used the local coolant temperatures and oxide thicknesses generated in a TASHA 95\% probability case for the G693 core design. The local meat thickness and relative power density were from the VENTURE analysis of the G693 core design. This analysis is a reasonable approximation taken in the absence of a new core design and was used investigate the modification of the fuel and to explore potential benefits of tightening some of the fuel plate inspection requirements.

Figures 8.3 and 8.4 compare the peaking factors and maximum temperatures from this LAT analysis with those from the G693 $95 \%$ probability LAT analysis. Although the reduced enrichment fuel reduces the maximum temperatures by about 5\%, they are still high. The maximum peaking factors were reduced by $3 \%$. This reduction was a little surprising since the reduced enrichment increased the size of the segregation volume. The increase in volume would increase the length of the lower conductivity heat transfer path from the center to the edge of the segregation volume. This was expected to increase the temperature center temperature. 


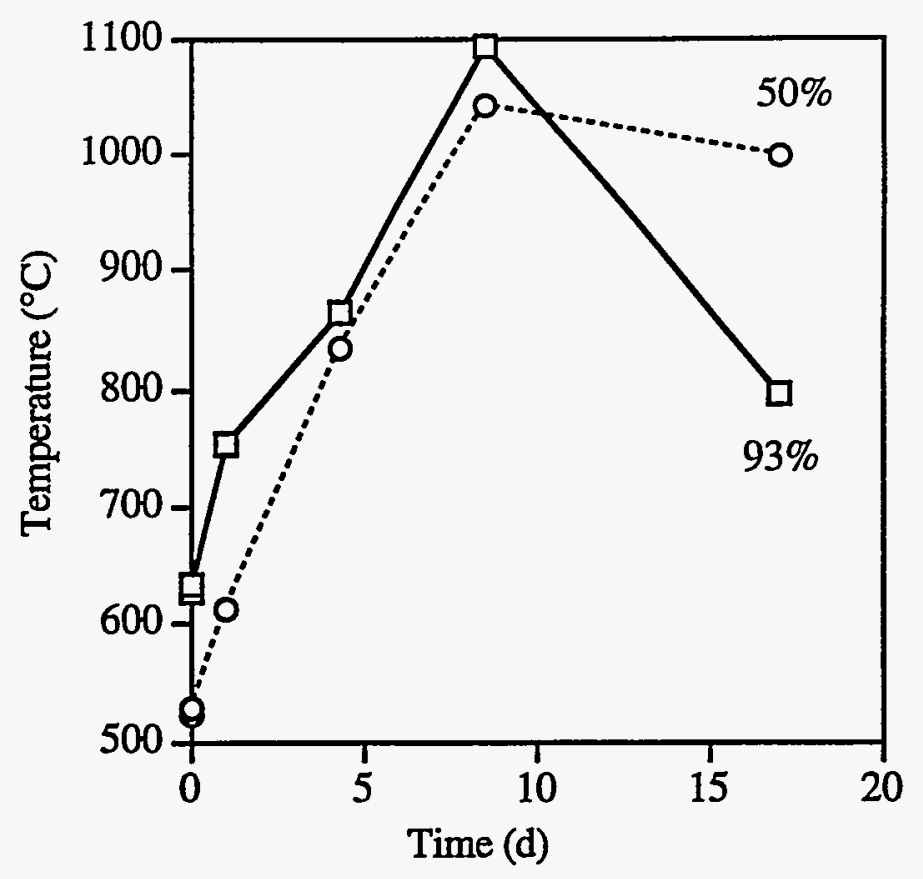

Fig. 8.3. Comparison of maximum temperatures from LAT analysis, 95\% limits, G693, high-enriched and medium-enriched fuel.

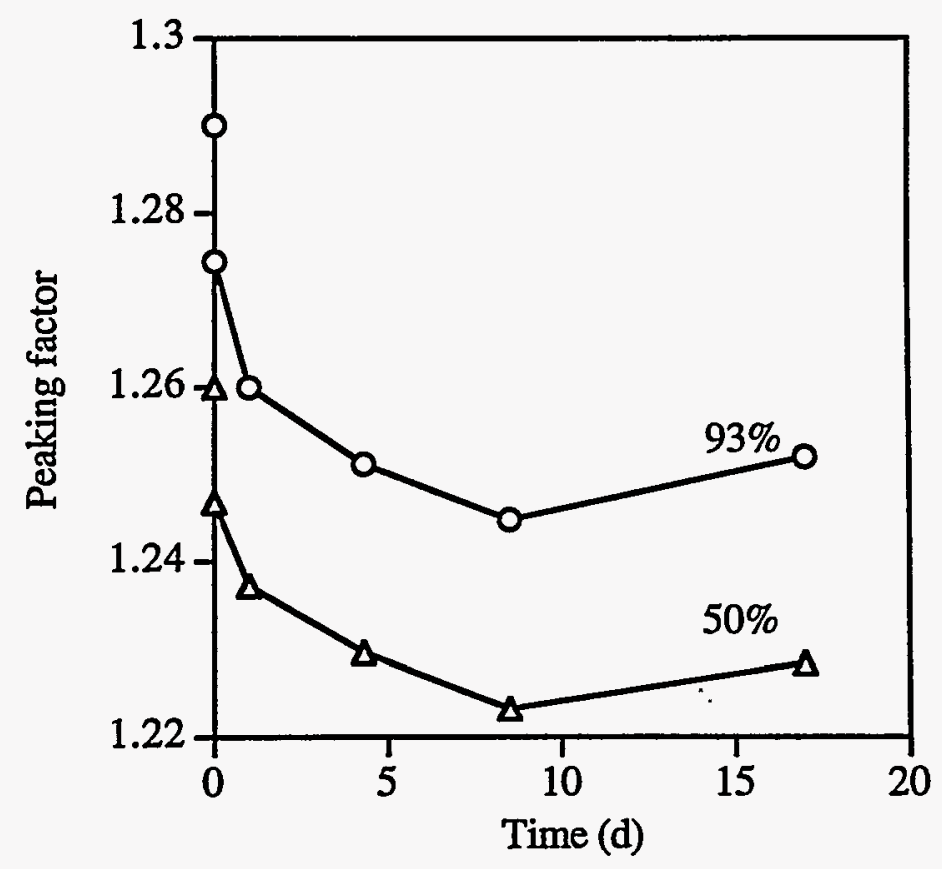

Fig. 8.4. Comparison of maximum peaking factors from LAT analysis, 95\% limits, G693, high-enriched and medium-enriched fuel. 
The ANSR excess fuel loading inspection spot size was taken from the HFIR testing procedures. This procedure was set before the HFIR was built based on the then current technology. Advances in technology demonstrated in Europe indicate that the inspection spot size could be reduced to a 0.4 by $0.54 \mathrm{~mm}$ spot from the 2-mm-diam circular spot. The $20 \%$ fuel overload factor (FO) is also based on old technology. It was speculated that this criterion could be reduced. The potential benefit of this reduction was estimated as a guide to judging if it would be cost effective to spend the development funds on tightening this inspection specification. The results of these studies are summarized in Figs. 8.5 and 8.6. The discontinuous behavior of the maximum temperature in Fig. 8.5 demonstrates the significance of the assumed transition temperature for the conversion of $\mathrm{U}_{3} \mathrm{Si}_{2}$ into $\mathrm{U}(\mathrm{Al}, \mathrm{Si})_{3}$. Although an inspection spot diameter of $1.4 \mathrm{~mm}$ produces this jump in maximum temperatures, an FO of $100 \%$ alone is still not sufficient to produce the conversion of the fuel material for a $0.54 \mathrm{~mm}$ diam. inspection spot size.

The small peaking factors and maximum temperatures produced by using the smallest inspection spot size prompted a revisit to the issue of centering the fuel meat within the fuel volume. It was discovered that with the smallest inspection spot, $F O=0.20$, the maximum peaking factor for a noncentered fuel meat was 1.175. Although this is higher than for a centered fuel meat (1.075), it is lower than the maximum peaking factor for the standard inspection specifications (1.26). These results were produced in a modified LAT G693 core design, $95 \%$ probability case. Other conditions may have different conclusions, but the need for centering should be reexamined as the core design matures.

\subsection{TEMPERATURE DEPENDENCY OF FUEL CONDUCTIVITIES}

The current model in the DART code assumes all the material is isothermal. The material in the model is assumed to remain at the initial temperature during the irradiation cycle. As has been shown, this is not the situation in the ANSR fuel. Also because of the internal heat generation in the fuel particle, the interior of a fuel particle is hotter than the surface. Although many of the chemical changes occur on the surface of the fuel particles, the interior temperatures do affect many of the conditions in the analysis. These effects are not accounted for in the current DART model. Unfortunately, development has been halted before a more detailed model incorporating these thermal effects as well as new experimental results could be developed. 


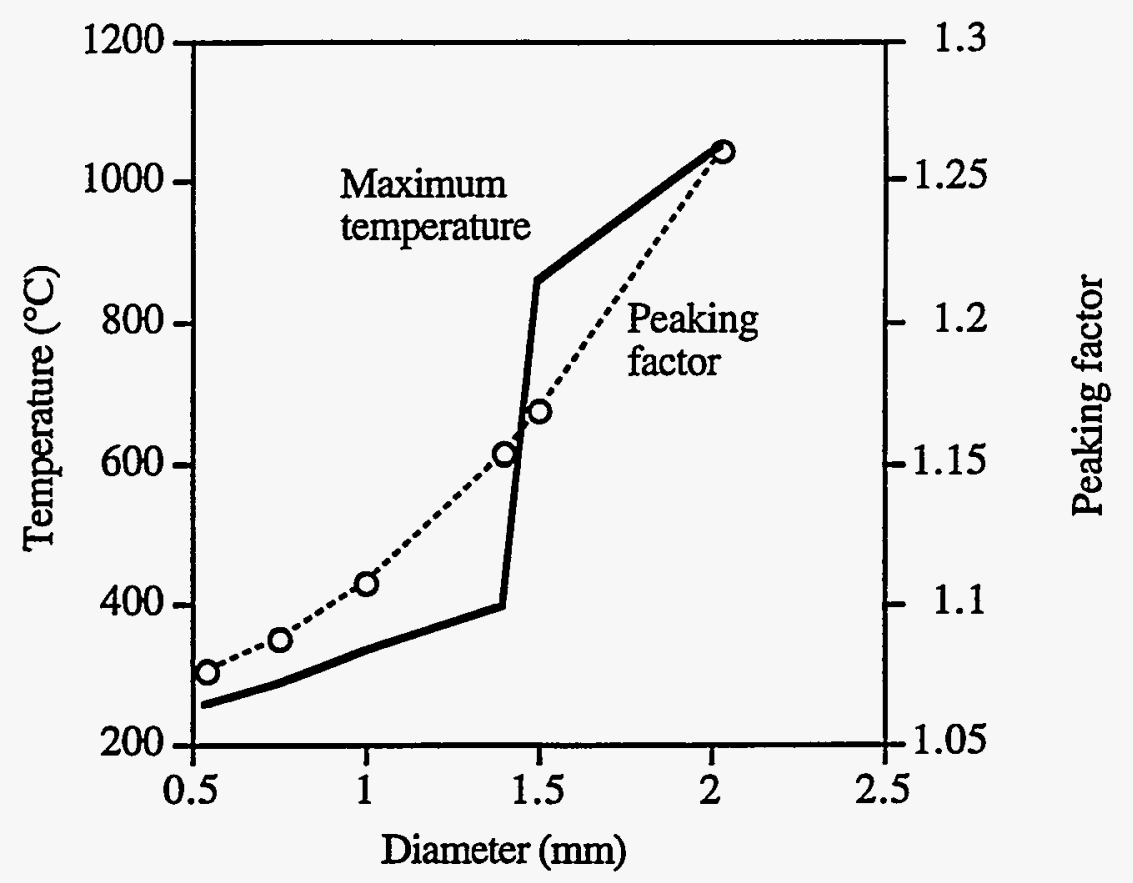

Fig. 8.5. Maximum temperatures and peaking factors as a function of diameter of the excess fuel loading inspection spot. Fuel overload $=20 \%$.

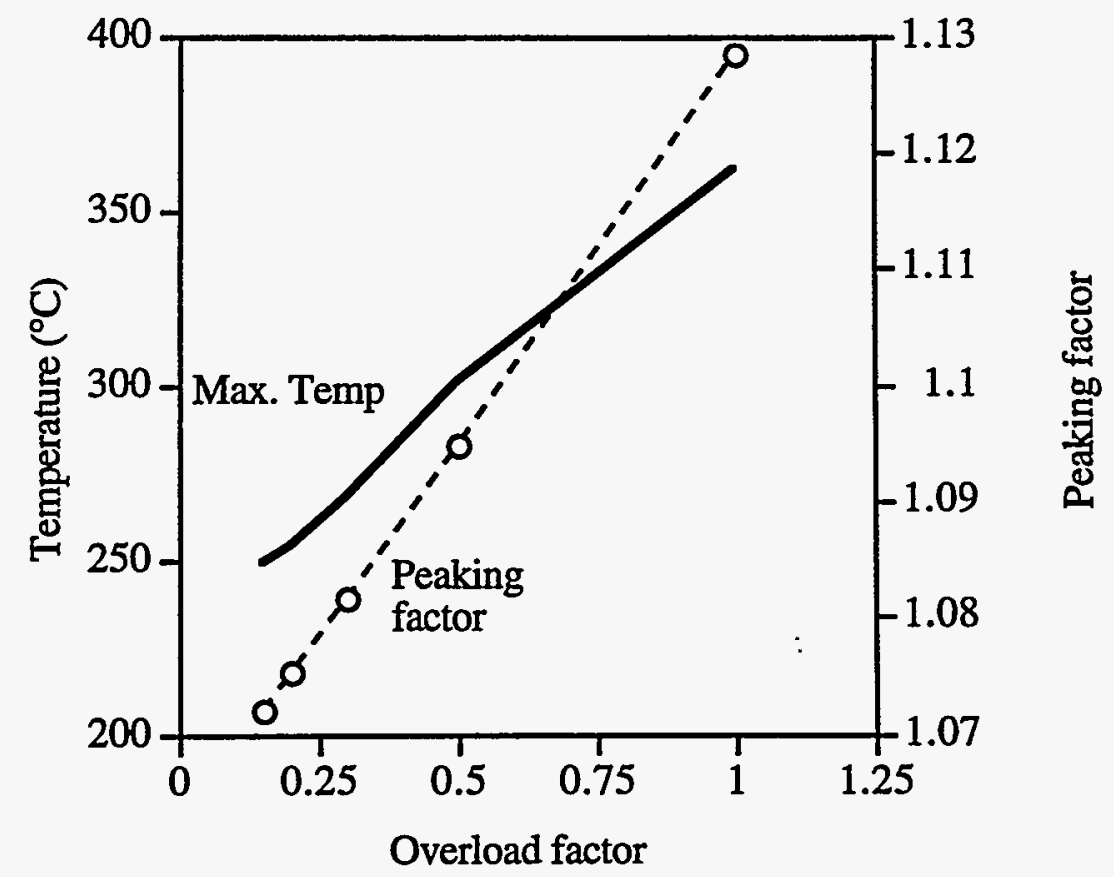

Fig. 8.6. Maximum temperatures and peaking factors as a function of the excess fuel loading overload factor. Segregation inspection spot diameter $=0.54 \mathrm{~mm}$. 
Initial investigations of the temperature dependencies of the fuel conductivities were conducted using the modified G693 LAT analyses. Again, these modified G693 analyses were used as reasonable approximations even though they did not represent an actual core design. Figure 8.7 presents the results of DART analyses for thermal conductivities of normal fuel with three different isothermal assumptions. Figure 8.8 presents a comparison of the maximum temperatures. Although there are significant differences between the conductivities produced using $473 \mathrm{~K}$ and $573 \mathrm{~K}$ as the isothermal temperature, the maximum temperatures show very little difference. There is a large difference between the $673 \mathrm{~K}$ case and the others. The major reason for this difference is the discontinuous effect of the $\mathrm{U}(\mathrm{Al}, \mathrm{Si})_{3}$ conversion transition temperature assumption. These calculations are very preliminary but are presented to demonstrate the complex behavior of the thermal conductivity of these cermets and to encourage more research. Precise determination of the thermal characteristics of these materials and the interaction of the chemical reactions with temperature and fission density will be require to improve the estimates of the thermal performance of these fuels. The large range in thermal conductivity and the complex interactions within the thermal environment make it very difficult to determine a conductivity value that will always be conservative for all conditions.

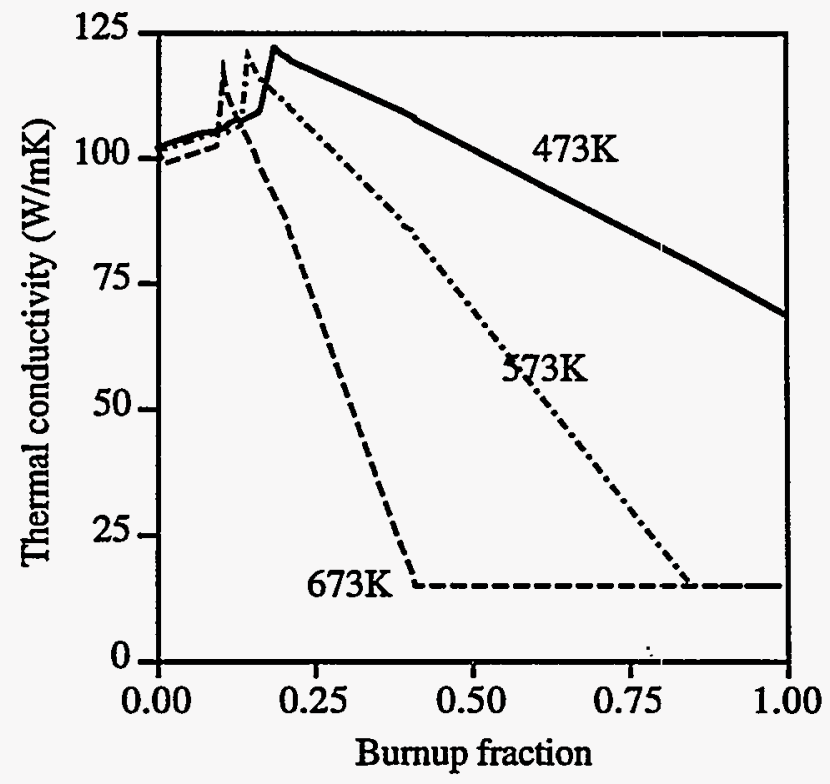

Fig. 8.7. DART thermal conductivity estimates for medium-enriched normal fuel as a function of isothermal temperature assumption. 


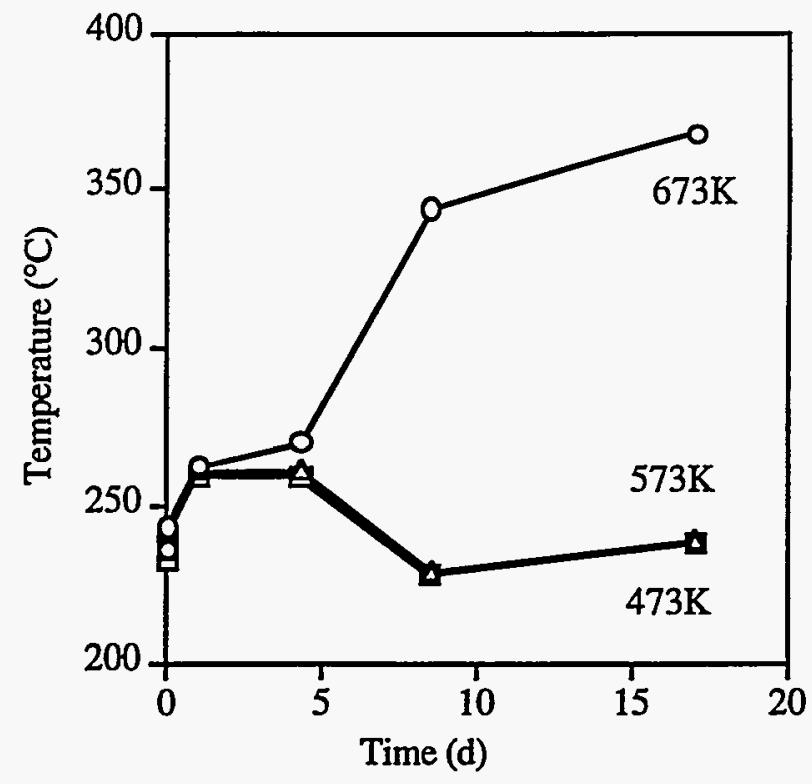

Fig. 8.8. Comparison of maximum temperatures for LAT analyses of the modified G693, 95\% limits case using DART produced thermal conductivities of the normal fuel with different isothermal temperature assumptions. 



\section{CONCLUSIONS}

It is difficult, if not impossible, to determine a single set of thermal parameters that will produce strictly conservative analyses. Conservative analyses produce results that are worse than would be experienced under the most severe possible conditions. Although these types of conservative assumptions have been made in the past, more detailed information, for example, on the complex phenomena that affect the thermal conductivity make the conservatism of those analyses less certain.

It is possible to analyze the ANSR fuel plate defects in a detailed manner incorporating estimates of the local conditions for thousands of data points and several time steps. This LAT can produce more accurate but still conservative estimates of the maximum peaking factors and temperatures in a specific design. These more accurate analyses can allow improved predictions of ANSR thermal hydraulic performance.

Tighter inspection could reduce the temperatures and peaking factors substantially. Even with tighter inspection, LAT is still needed due to the complex behavior of the fuel thermal conductivity as a function of fission density, temperature and $\mathrm{U}_{3} \mathrm{Si}_{2}$ conversion. The meat thickness variation analyses can't determine effects of the fuel conversion process.

The DART thermal conductivity model should be extended to incorporate the internal temperature of the fuel particles and a nonuniform temperature field across the model. This more complex model should be incorporated into the LAT to give more accurate representation of the thermal behavior.

Details on the conversion of the $\mathrm{U}_{3} \mathrm{Si}_{2}$ fuel into $\mathrm{U}(\mathrm{Al}, \mathrm{Si})_{3}$ should be developed. Determining the effect of temperature on the conversion rate and completeness would be required to incorporate a more accurate model into the LAT. 


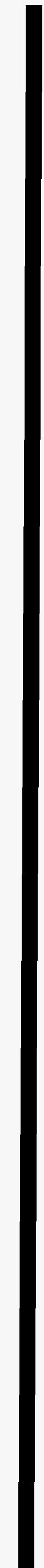




\section{REFERENCES}

1. B. W. Pace, G. L. Copeland, Fabrication Development for the Advanced Neutron Source Reactor, ORNL/TM-13070, Oak Ridge National Laboratory, Oak Ridge, Tenn., September 1995.

2. N. Hilvety, T. G. Chapman, HFIR Fuel Element Steady State Heat Transfer analysis, ORNL-TM-1903, Oak Ridge National Laboratory, Oak Ridge, Tenn., December 1967.

3. J. R. Kirkpatrick, Calculations for HFIR Fuel Plate Non-bonding and Fuel Segregation Uncertainty Factors, K/CSD/TM-79, Martin Marietta Energy Systems, Inc, Oak Ridge Tenn., October 1990.

4. Howard A. McLain, HFIR Fuel Element Steady State Heat Transfer Analysis Revised Version, ORNL-TM-1904, Union Carbide Corp., Nuclear Div., Oak Ridge National Laboratory, Oak Ridge, Tenn., December 1967.

5. J. R. Kirkpatrick, "Preliminary Results for ANS Fuel Plate Peaking Factors for Non-bonds", Internal correspondence to G. L. Yoder, September 18, 1989.

6. J. R. Kirkpatrick, "Preliminary Results for ANS Fuel Plate Peaking Factors for Non-bonds - Addendum: Peak Temperatures for Low Power Level," Internal correspondence to G. L. Yoder, September 21, 1989.

7. B. S. Petukhov, "Heat Transfer and Friction in Turbulent Pipe Flow with Variable Physical Properties," Advances in Heat Transfer, Vol. 6, 1970, p. 528.

8. G. E. Giles, Internal correspondence to G. L. Yoder, Oak Ridge National Laboratory, Oak Ridge, Tenn., October 1988.

9. G. E. Giles, "Thermal studies of ANS fuel plate - Distributed segregation assumption and centered meat fuel design," Internal correspondence to G. L. Yoder, October 9, 1991.

10. G. L. Yoder, Jr., compiler, Steady-State Thermal-Hydraulic Design Analysis of the Advanced Neutron Source Reactor, ORNL/TM-12398, Oak Ridge, Tenn., May 1994. 
11. J. Rest, "The DART Dispersion Analysis Research Tool: A Mechanistic Model for the Prediction of Fission-Product-Induced Swelling of Aluminum Dispersion Fuels.- Users Guide for Mainframe, Workstation, and Personal Computer Applications," to be published as an Argonne National Laboratory Report (1995).

12. J. Rest, J. L. Snelgrove, "An estimate of evolution of dispersion fuel thermal conductivity for hot spots with segregations $\left(50 \% \mathrm{U}_{3} \mathrm{Si}_{2}\right.$, $15 \%$ void)", Informal correspondence from J. L. Snelgrove, Argonne National Laboratory, to G. E. Giles, April 8, 1992.

13. Moshe Siman-Tov, "Physical Property Correlations for Water and Heavy Water," Internal correspondence to D. L. Selby, September 30, 1991.

14. D. R. Vondy, T B. Fowler, G. W. Cunningham, III, The BOLD VENTURE Computation System for Nuclear Reactor Core Analysis, Version III, ORNL-5711, Union Carbide Corp., Nuclear Div., Oak Ridge National Laboratory, June 1981.

15. J. L. Snelgrove, et al., Evaluation of Existing Technology Base for Candidate Fuels for HWR-NPR, ANL/NPR-93/002, Argonne National Laboratory, February 1983.

16. G. L. Hofman, "Some Recent Observations on the Radiation Behavior of Uranium Silicide Dispersion Fuel", Proceeding of the 1988 International Meeting on Reduced Enrichment for Research Reactors, ANL/RERTR/TM-13 CONF-8809221, September 1988.

17. "Minutes of the Workshop Meeting on ANS Fuel Plate Temperatures on January 27, 1994," Internal memo from George Copeland to Doug Selby, February 25, 1994.

18. J. Rest, Informal correspondence to G. E. Giles, October 5 and November 8, 1994.

19. J. W. Bryson, C. R. Luttrell, G. T. Yahr, "Structual Evaluation of Fuel Plate Debond," Monthly Progress Report for March 1994, ORNL/ANS/INT-5/V72, April 1994. 


\section{APPENDIX A}

\section{AUTOMATIC EXECUTION OF LAT ANALYSES}

The automatic execution of the LAT analysis is a three step process. In the first step a code, MK-FD, is run to generate a history of the fission density at each point. MK-FD is described in Appendix E. The output of MK-FD is a data set containing the fission densities for each data point. The second program, RD-ALL, uses this data set and others describing the local meat thickness, relative power densities, oxide thickness and bulk coolant temperatures. The meat thickness data are interpolated on the power density grid. The other data sets (except fission density) are inverted to adjust for the up-flow condition of the ANSR. These data are then written out to a new data set that contains the interpolated meat thickness and other data in the correct orientation to provide the input data for the LAT analyses. These codes must be run whenever the basic core design, number of time steps or the type of analysis (no-uncertainties, 95\% limits, etc.) is changed. They produced a pair of data sets, tecconu for the upper plate and tecconl for the lower plate.

The third step is to run the driver program RH7-ANSR. The driver program reads the two data sets in sequence, processes the data and then generates an input file for a HEATING analysis. A modified HEATING executable is called by the driver. This modification has user-supplied routines to report the required information and determine the nonlinear conductivities. The resulting HEATING output file is processed to extract information about the peaking factors, maximum temperature, maximum extent of the $\mathrm{U}(\mathrm{Al}, \mathrm{Si})_{3}$ cylinder, and various other data that are recorded in the output file $h p f$. This file is the main result of the LAT analysis. All other HEATING-produced and temporary files are deleted at the end of the analysis for each point. The file $h p f$ can be further processed to identify the maximum peaking factor, segregated and normal fuel temperatures, and oxide thickness as a function of time. It can also be used in TECPLOT to produce contour plots of peaking factors, temperatures and oxide thickness. The input parameters can also be displayed in this fashion.

The input and output files of the program RD-ALL are described in the sections of this appendix. 


\section{A. 2}

\section{A.1 RD-ALL INPUT FILES DESCRIPTIONS}

\section{A.1.1 Meat Thickness}

The meat thickness distribution is taken from the VENTURE input data set. The format of this data set is described in Table A.1. The mesh for this data is coarser than the relative power density data set as shown in Figs. 4.6 to 4.7. Appendix D contains contour plots of the meat thickness distribution for both the L7 and G693 core designs.

Table A.1. Format of the input meat thickness distribution data set

\begin{tabular}{|c|c|c|}
\hline \multicolumn{3}{|c|}{ Line 1-end Three entries in free-form format } \\
\hline Entry & Variable & Units \\
\hline 1 & TM & $(\mathrm{mm})$ \\
\hline & Meat thickness & \\
\hline 2 & $\mathbf{R}$ & $(\mathrm{mm})$ \\
\hline 3 & $\begin{array}{c}\text { Radius } \\
Z \\
\text { Axial location }\end{array}$ & $(\mathrm{mm})$ \\
\hline
\end{tabular}
A.1.2 Relative Power Density
The relative power density is obtained from the VENTURE code. This code produces power densities for each node for every time step. The format of one time step is described in Table A.2. The block of data is repeated for every time step. Data for both the upper and lower plates are included in each time step. For the G693 core design, the grid had 48 (radial) by 69 (axial) $=3,312$ grid points distributed over both plates.
The data from VENTURE have a small error in the axial locations of the upper core data points. The upper core is output in the inverted position. This error is corrected in RD-ALL. Only the upper-core data points are adjusted. 


\section{A.3}

Table A.2

Format of a Time Step Block in the Relative Power Density Data set

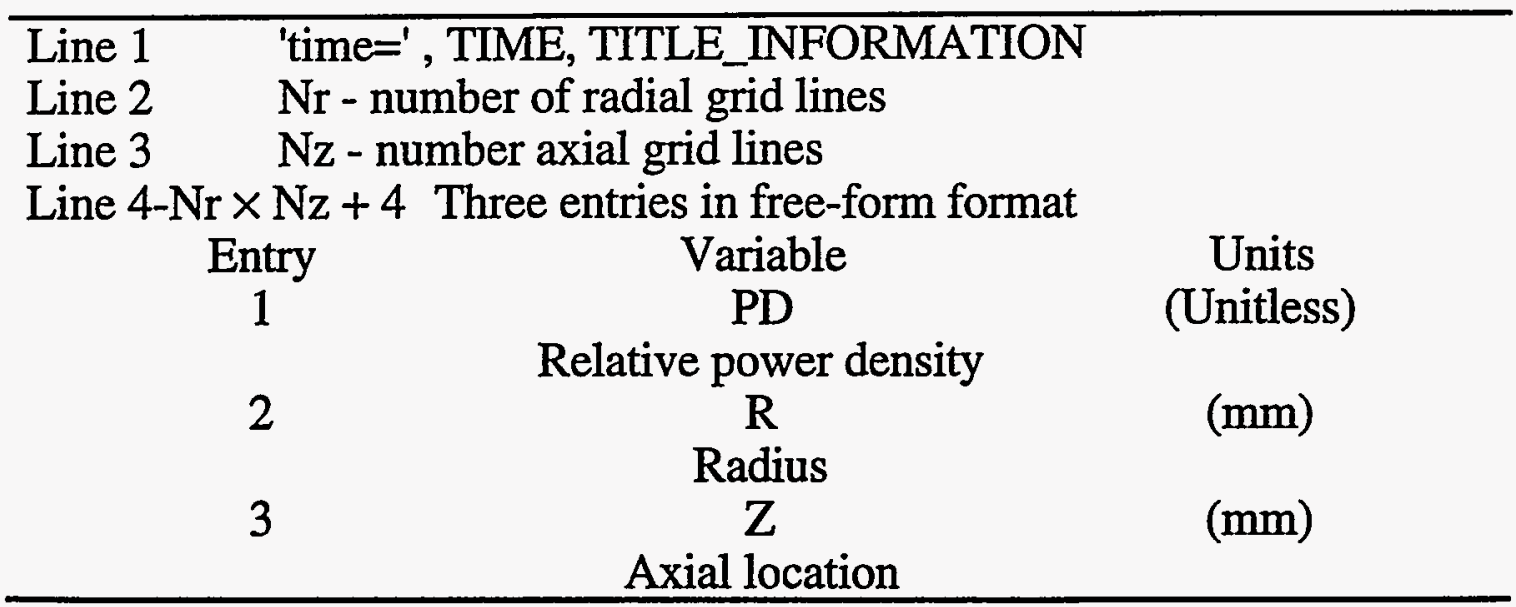

\section{A.1.3 Oxide Thickness}

The oxide thickness is reported by the TASHA code and is in an inverted orientation that is corrected in the program RD-ALL. The format of this data set is described in Table A.3.

The TASHA code is a modification of the HFIR steady-state code. In HFIR downflow is used to cool the core. As an expediency in the development of TASHA, the core coordinate system is inverted to simulate the upflow condition of ANSR. This required modification of the $\mathrm{Z}$ locations of the oxide thickness and coolant temperature data points is made to be consistent with the meat thickness, power density and fission density data sets.

Table A.3

Format of a Time-Step Block in the Oxide thickness Data set

\begin{tabular}{ccc}
\hline Line 1 & 'time=', TIME, Nu, N1, TITLE_INFORMATION \\
$\begin{array}{c}\text { Line 2-Nu+N1+2 } \\
\text { Entry }\end{array}$ & $\begin{array}{c}\text { Three entries in free-form format } \\
\text { Variable }\end{array}$ & $\begin{array}{l}\text { Units } \\
\text { OX }\end{array}$ \\
2 & Oxide thickness & $(\mu \mathrm{m})$ \\
$\mathrm{R}$ & $(\mathrm{mm})$ \\
3 & $\begin{array}{c}\text { Radius } \\
\text { Axial location }\end{array}$ & $(\mathrm{mm})$ \\
\hline
\end{tabular}




\section{A.4}

\section{A.1.4 Coolant Bulk Temperature}

The local coolant temperature is produced by the TASHA code and it is in the same order as the oxide thickness data set and requires the correction for the up-flow orientation of ANSR. The format of this data set is described in Table A.4.

Table A.4

Format of a Time Step Block in the Coolant Temperature Data set

Line 1

Line $2-\mathrm{Nu}+\mathrm{Nl}+2$

Entry

2

3 'time $=$ ' , TIME, Nu, Nl, 'TITLE_INFORMATION

Three entries in free-form format

Units

(mm)

(mm)

Axial location

\section{A.1.5 Fission Density}

The fission density is obtained from the relative power density produced by the VENTURE code. The technique for integrating the power density is described in Appendix E. The format of one time step of this data set is also described in Table A.5. This block of data is repeated for every time step. Data for both the upper and lower plates are included in each time step. For the G693 core design, the grid had 48 (radial) $\times 69$ (axial) $=3,312$ grid points distributed over both plates. The output of the MK-FD has been corrected for the VENTURE location error, so it is not adjusted in RD-ALL. 
Format of a Time-Step Block in the Fission Density Data set

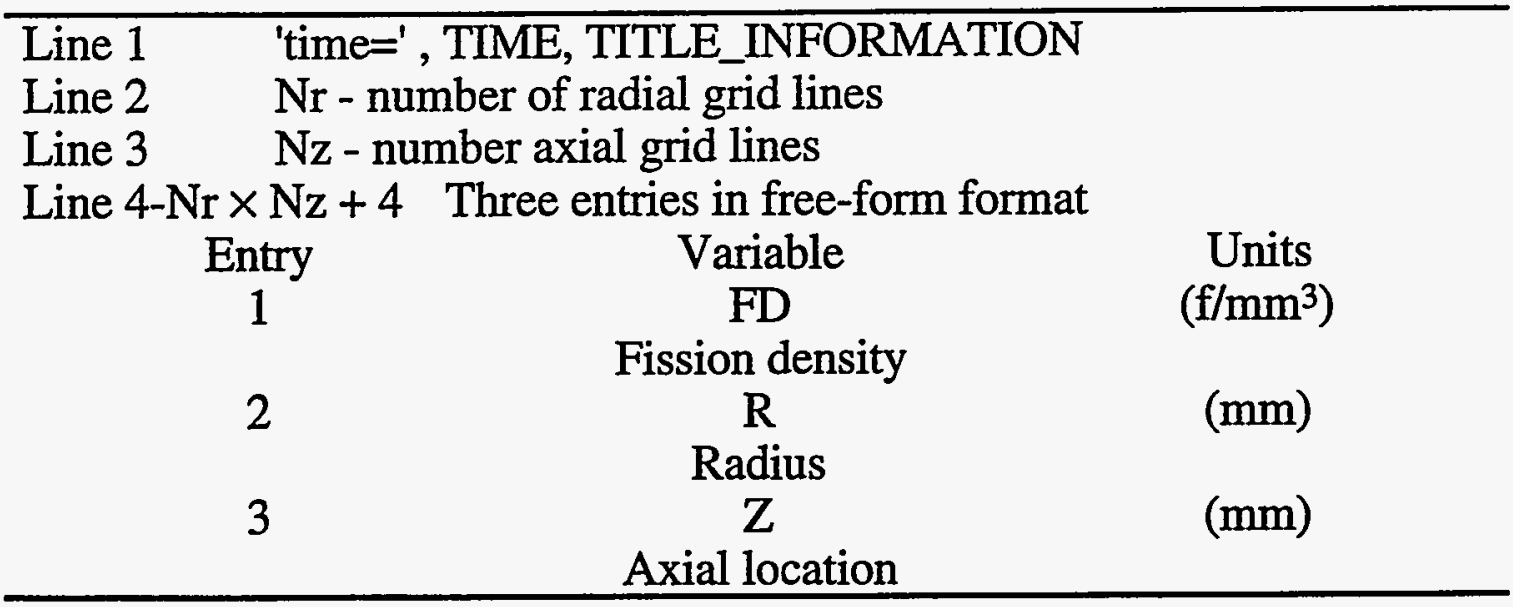

\section{A.2 RD-ALL OUTPUT FILE DESCRIPTION}

The primary output of the RD-ALL program are the file tecconl (lower core) and tecconu (upper core) which are used as input in LAT. The format of these files is described in Table A.6.

Table A.6. Format of tecconl and tecconu files

Line 1

Line 2

Line 3

Line 4-Nu+Nl+4

Entry

1

2

3

4

5

6 'title=' , TITLE_INFORMATION

'variables $=\mathrm{r} \mathrm{z}$ pd cd cdr fd tm ox tb'

'zone $T=$ ',TIME, ', i=69, j=24, f=point'

Nine entries in free-form format

Variable

$\mathbf{R}$

Radius

$\mathrm{Z}$

Axial location

PD

Relative power density

$\mathrm{CD}$

Thermal conductivity of

Units

(mm)

(mm)

(Unitless)

$\left(\mathrm{W} / \mathrm{cm}^{\circ} \mathrm{C}\right)$

normal fuel

CDR

Thermal conductivity of segregated fuel

FD

$\left(\mathrm{f} / \mathrm{mm}^{3}\right)$

Fission density 
A. 6

Table A.6 Format of tecconl and tecconu files (continued)

\begin{tabular}{ccc}
\hline 7 & TM & $(\mathrm{mm})$ \\
8 & Meat thickness & OX \\
Oxide thickness & $(\mu \mathrm{m})$ \\
9 & TB & $\left({ }^{\circ} \mathrm{C}\right)$ \\
\hline
\end{tabular}

\section{A.3 RD-ALL CODE LISTING}

The program RD-ALL is listed in Table A.7. It was developed to run on an IBM RISC 6000 using FORTRAN version xlf 2.3. 


\section{A.7}

\section{Table A.7. Code listing of RD-ALL}

C

C

C

C

C

c

c

c

100 continue $\&$ $\&$

$\mathbf{C}$

this code reads the pd, tm, ox, and fd tables. it writes out a table of $r, z, t m, f d, o x$, and pd and a table of $r, z, p d, t m, c d, c d r$, and ox in a form that can be used in the h7run script to run a h7.2 job for each data point.

parameter (maxpds $=4000, \operatorname{maxp} 2=1000)$

dimension $r$ (maxpds), $z$ (maxpds), fd (maxpds), pd (maxpds), cd (maxpds), $\mathrm{cdr}$ (maxpds), ox (maxpds), nr (maxpds) , $\mathrm{nz}$ (maxpds), $r c(\operatorname{maxp} 2), d r(\operatorname{maxp} 2), z c(\operatorname{maxp} 2), t m(\operatorname{maxp} 2), d z(\operatorname{maxp} 2)$, tb (maxpds)

common /pdgrid/mxru,mxzu, mnru,mnzu, mxrl, mxzl, mnrl,mnzl

character cline* 80, label, equal

data tmmax/0.7366/,isort/0/,itime/1/

open data sets

open (unit =5, file=' $/$ home $/$ geg $/$ anal $/ \mathrm{a} /$ fy $94 /$ tables $/ g 693 / g 693-t m$ ' status='old')

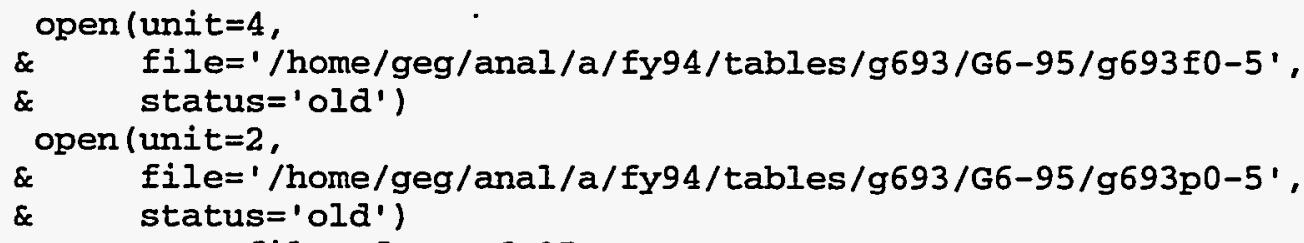

output tecplot tables of all variables

write $(10, *)$ 'title="g6be lc rzpccfto $10 / 7 / 93$ "'

write $(10, *)$ 'variables $=r z$ pd cd cdr fd tm ox tb'

write $(11, *)$ 'title="g6be uc rzpccfto $10 / 7 / 93$ "'

write $(11, *)$ 'variables $=r$ pd cd car fd tm ox tb'

read the $f d$, power density and oxide thickness for every time step write out in data set 


\section{Table A.7. Code listing of RD-ALL (continued)}

c

iutec $=m \times r u-m n r u+1$

jutec $=m x z u-m n z u+1$

iltec $=m \times r l-m n r l+1$

jltec $=\operatorname{mxz} 1-\mathrm{mnz} l+1$

c

c

C

C

C

C

c

C

c

C

C

C

do 5000 it $=1,6$

if(it.eg.2) then

rewind 2

itime $=1$

endif

c

C

$\&$

$\&$

if(it.eq.2) rewind 4

do $4000 i=1,1 p d$

six time steps are used

read the next power density time step

modified to calculate same as tasha $10 / 25 / 93$

call rdpd (pdtim, pd, lpd, r, z, itime)

if(it.eq.6) call rdpd(pdtim,pd,Ipd,r,z,itime)

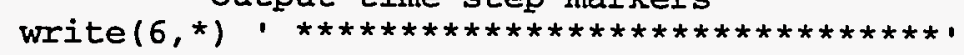

write $(6, *)$ ' Time step $=$ ',it, ' pdtim=',pdtim

write $(10$, ' ('zone $T=" t='$ ', $\{5.2, '$ ' , $i=69, j=24, F=$ POINT' ')')

write (11, '( ' 'zone $T=" t=$ ' , f5.2,' ", $i=69, j=24, F=$ POINT' ')') pdtim

read the next fd time step

read the next power density time step

modified to calculate same as tasha $10 / 25 / 93$

call rafd (fdtim, fd, lpd)

if(it.eq.6) call rafd(fdtim,fd,Ipd)

read the next oxide thickness time step

the tasha oxide data has only 5 time steps

if(it.gt.1) call rdox(oxtim, ox, lpd, r, z)

read the next bulk temperature time step

cal1 ratb(tbtim, tb, 1pd, r, z)

calculate conductivity from fd

call calcds (fd, cd, cdr, Ipd)

find max and min of variables in upper and lower core

call fdmnmx (fd,pd, tm, cd, cdr, ox, tb, lpd, r, z, ltm)

sort if isort ne 0

if(isort.ne.0) call sort(isort, fd,pd, cd, cdr,ox,r,z, Ipd)

find the meat thickness at the pd point 
Table A.7. Code listing of RD-ALL (continued)

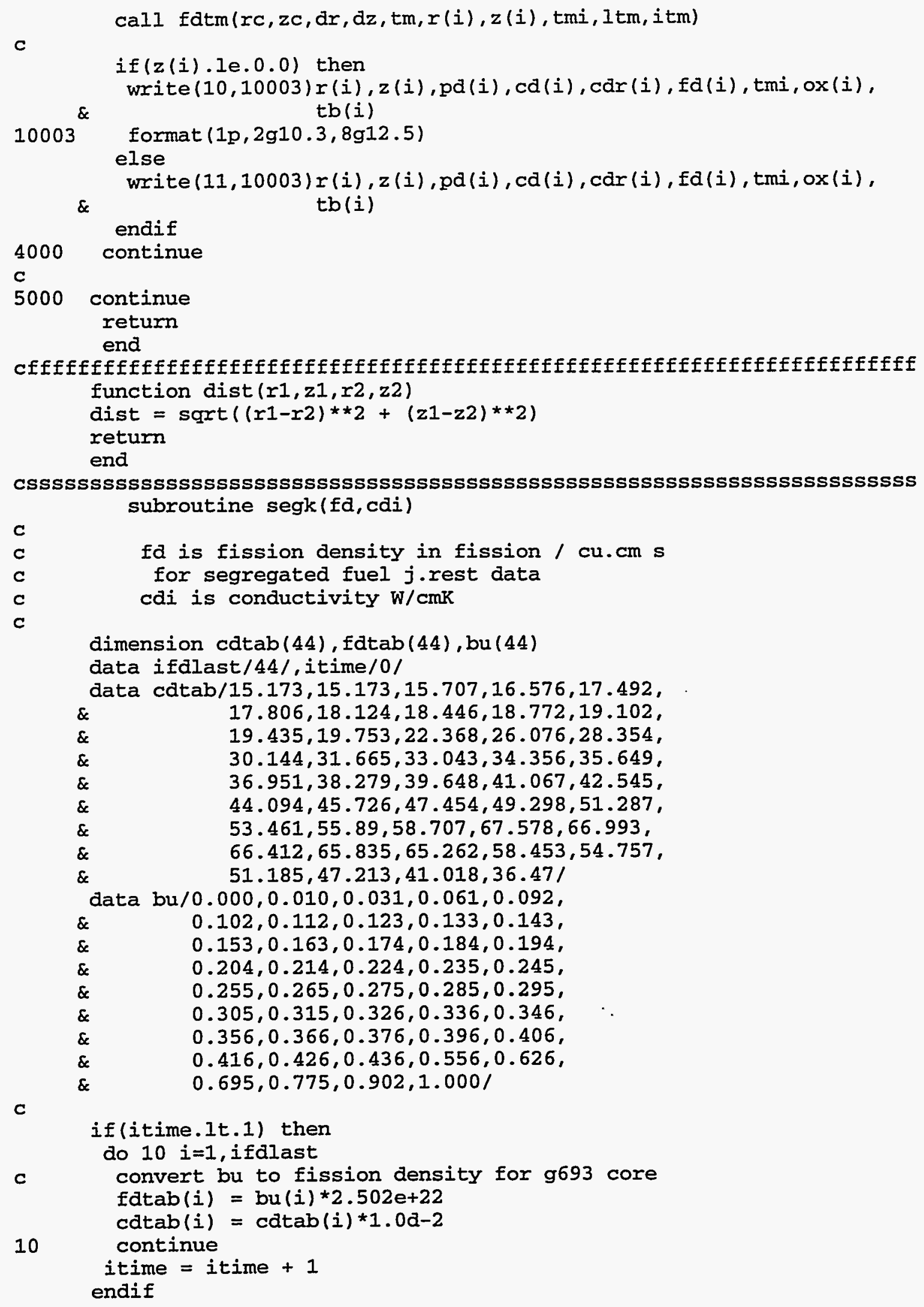




\section{A. 10 \\ Table A.7. Code listing of RD-ALL (continued)}

c

if(fd.1t.0.0) then

write $(6, *)$ ' error in table read $f d<0$ '

stop

endif

c

imatch $=0$

do $100 i=1$, ifdlast

if(fd.ge.fdtab(i)) imatch $=i$

100 continue

if (fd.ge.fatab(ifdlast)) then

fdratio = fd/fdtab (ifdlast)

write $\left(6,{ }^{*}\right)$ ' fd=maximum(seg) was',fd, fdratio

endif

$i=$ imatch

if(i.le.0) then

c if $\mathrm{fd}=0$ then $\mathrm{cd}=\mathrm{cdo}$

$\operatorname{cdi}=\operatorname{cdtab}(1)$

else if(i.ge.ifalast) then

$\mathbf{c}$

if $\mathrm{fd}>\max$ then $\mathrm{cd}=\mathrm{cd}$ \& $\mathrm{fdmax}$

cdi $=\operatorname{cdtab}(i f d l a s t)$

else

$\operatorname{cadi}=\operatorname{cdtab}(i)+(\operatorname{cdtab}(i+1)-\operatorname{cdtab}(i))$

$\&$

*(fd-fdtab(i))/(fdtab(i+1)-fdtab(i))

endif

c

return

end

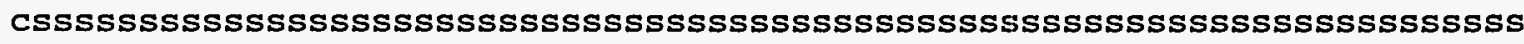
subroutine normk ( $\mathrm{fd}, \mathrm{cdi}$ )

C

c

c

C

C

C

dimension catab(23), fdtab (23), bu (23)

fd is fission density in fission / cu.cm s

for normal fuel - DART data

cdi is conductivity $\mathrm{W} / \mathrm{cmK}$

j-rest data for 3.5 fuel loading $50 \%$ enrich $10 / 10 / 94$

data catab/101.8,102.07,102.83,104.07,105.42,105.9,106.39,106.89,

\& $\quad 107.41,107.95,108.5,109.06,115.06,121.69,120.54,119.66$,

$\&$

$118.92,108.91,108.28,107.66,107.04,78.501,68.168 /$

burnup fraction taken from $j$ rest data

C

data bu/0.0,0.010,0.031,0.061,0.092,0.102,0.112,0.123,0.133,

$\& \quad 0.143,0.153,0.163,0.174,0.184,0.194,0.204,0.214,0.386$,

$\&$ $0.396,0.406,0.416,0.849,1.000 /$ :

data itime/0/

C

if(itime.eq.0) then

imaxt $=23$

itime $=1$

do $10 i=1$, imaxt

c convert bu to fission density for g693 core

fatab $(i)=b u(i) * 2.502 e+22$

fdtab $(i)=\operatorname{fdtab}(i) * 1.0221$

endif

if(fd.1t.0.0) then 


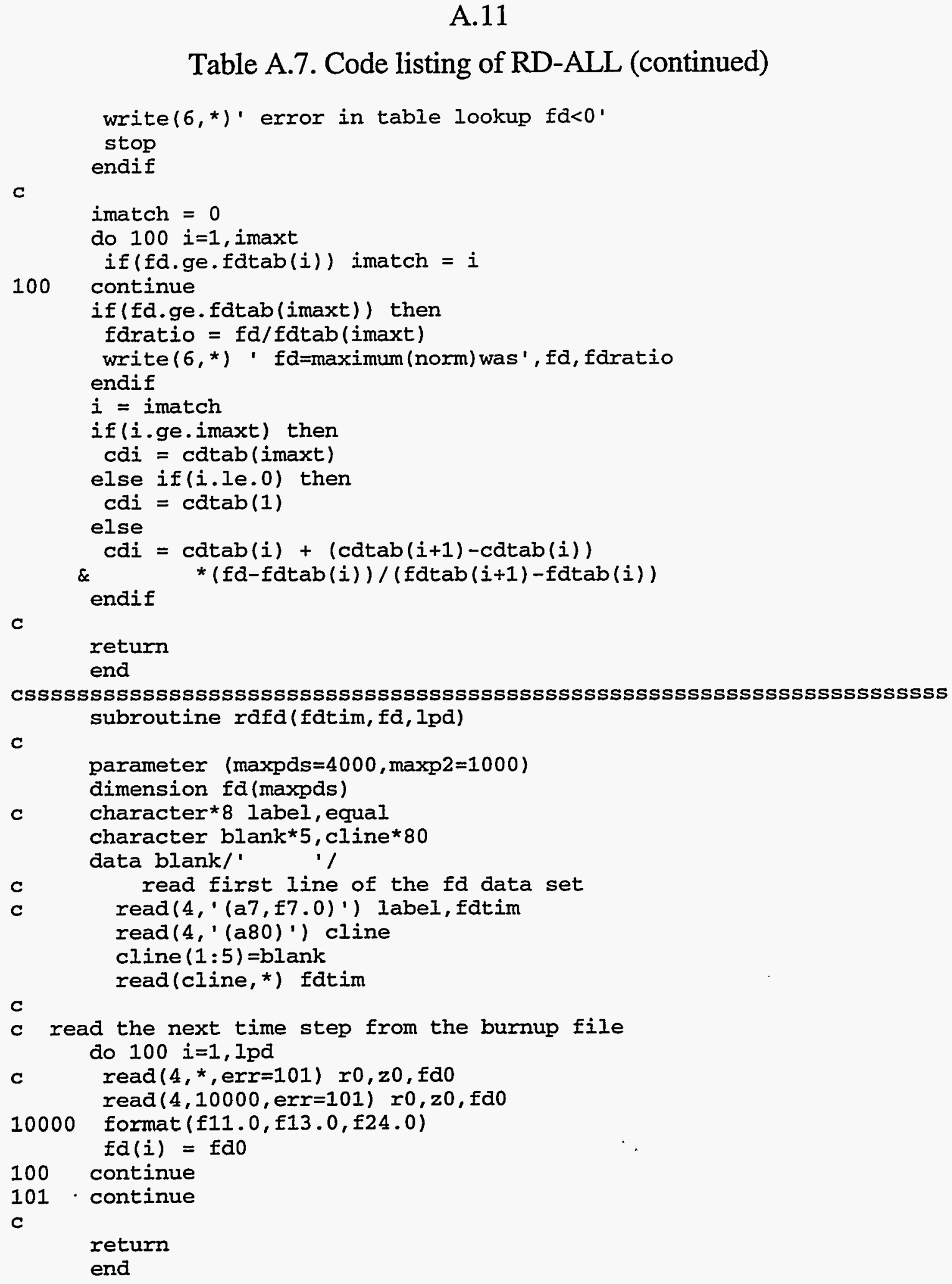




\section{A.12 \\ Table A.7. Code listing of RD-ALL (continued)}

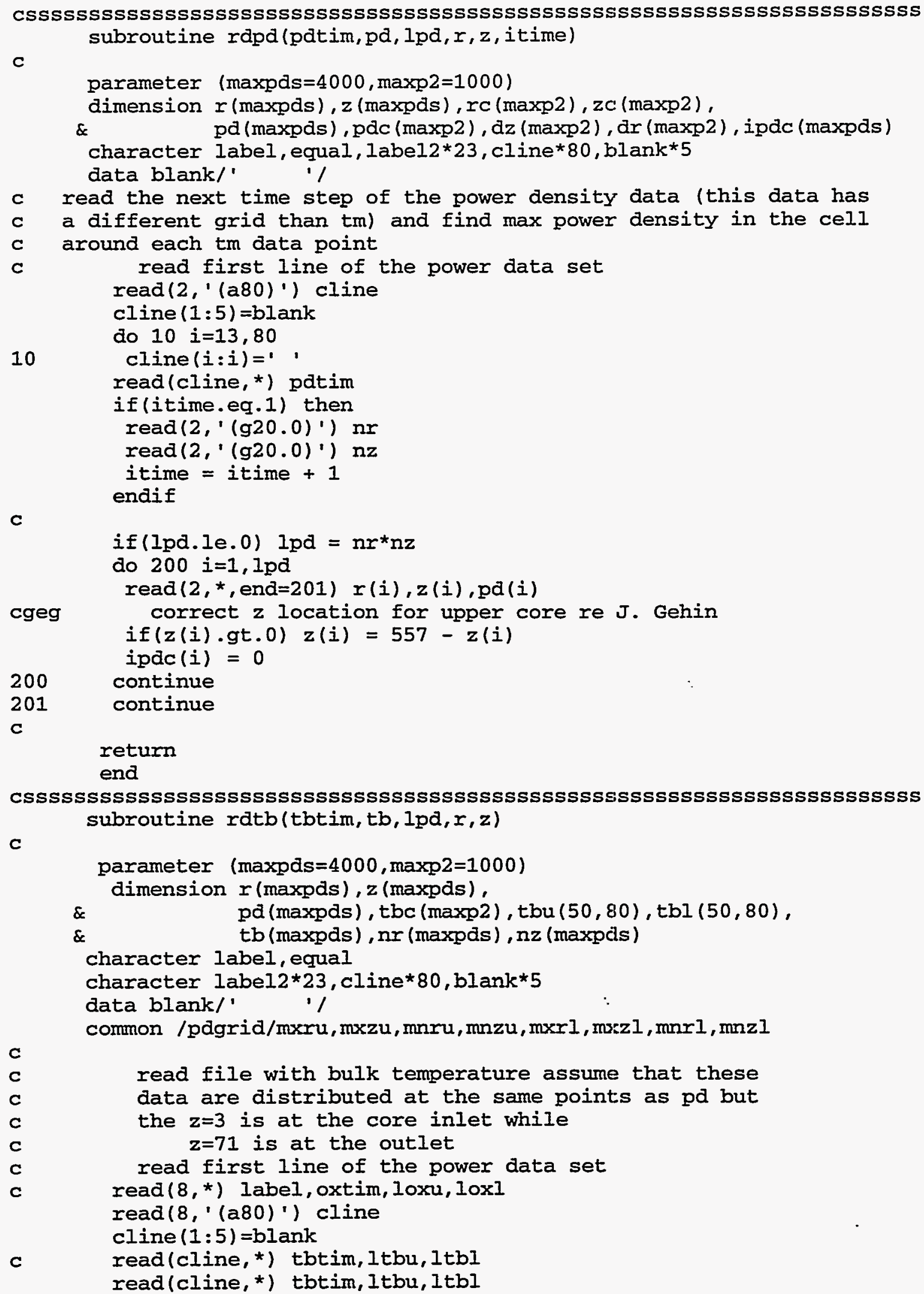

read file with bulk temperature assume that these

data are distributed at the same points as pd but

the $z=3$ is at the core inlet while $z=71$ is at the outlet

read first line of the power data set

read $(8, *)$ label, oxtim, loxu, loxl

read $\left(8, '(280)^{\prime}\right)$ cline

cline $(1: 5)=$ blank

read (cline, *) tbtim, ltbu, 1tbl

read(cline, *) tbtim, Itbu, Itbl 


\section{A. 13 \\ Table A.7. Code listing of RD-ALL (continued)}

c

$$
\begin{aligned}
& \operatorname{mxrl}=0 \\
& m \times z l=0 \\
& m n r l=1000 \\
& m n z l=1000 \\
& \text { do } 1100 \quad i=1,1 \text { tbl }
\end{aligned}
$$$$
\operatorname{read}(8, *) \mathrm{nr}(i), \mathrm{nz}(i), \mathrm{tbx}
$$$$
\text { if (nr(i).gt.0.and.nz(i).gt.0) then }
$$$$
\operatorname{tbl}(\mathrm{nr}(i), \mathrm{nz}(i))=\mathrm{tbx}
$$$$
i t b l=i
$$$$
\operatorname{mxx} I=\max (n x(i), \operatorname{mxr} l)
$$$$
\operatorname{mxzI}=\max (\mathrm{nz}(i), \operatorname{mxz} l)
$$$$
\operatorname{mnrl}=\min (n r(i), \operatorname{mnrl})
$$$$
\operatorname{mnzl}=\min (n r(i), \operatorname{mnrl})
$$$$
\text { else }
$$$$
\text { write }\left(6,{ }^{*}\right) \text { ' Error in rdtb:lc@i=',i,' } n r=1, n r(i), ' n z=', n z(i)
$$$$
\text { return }
$$$$
\text { endif }
$$

1100 continue

C

c

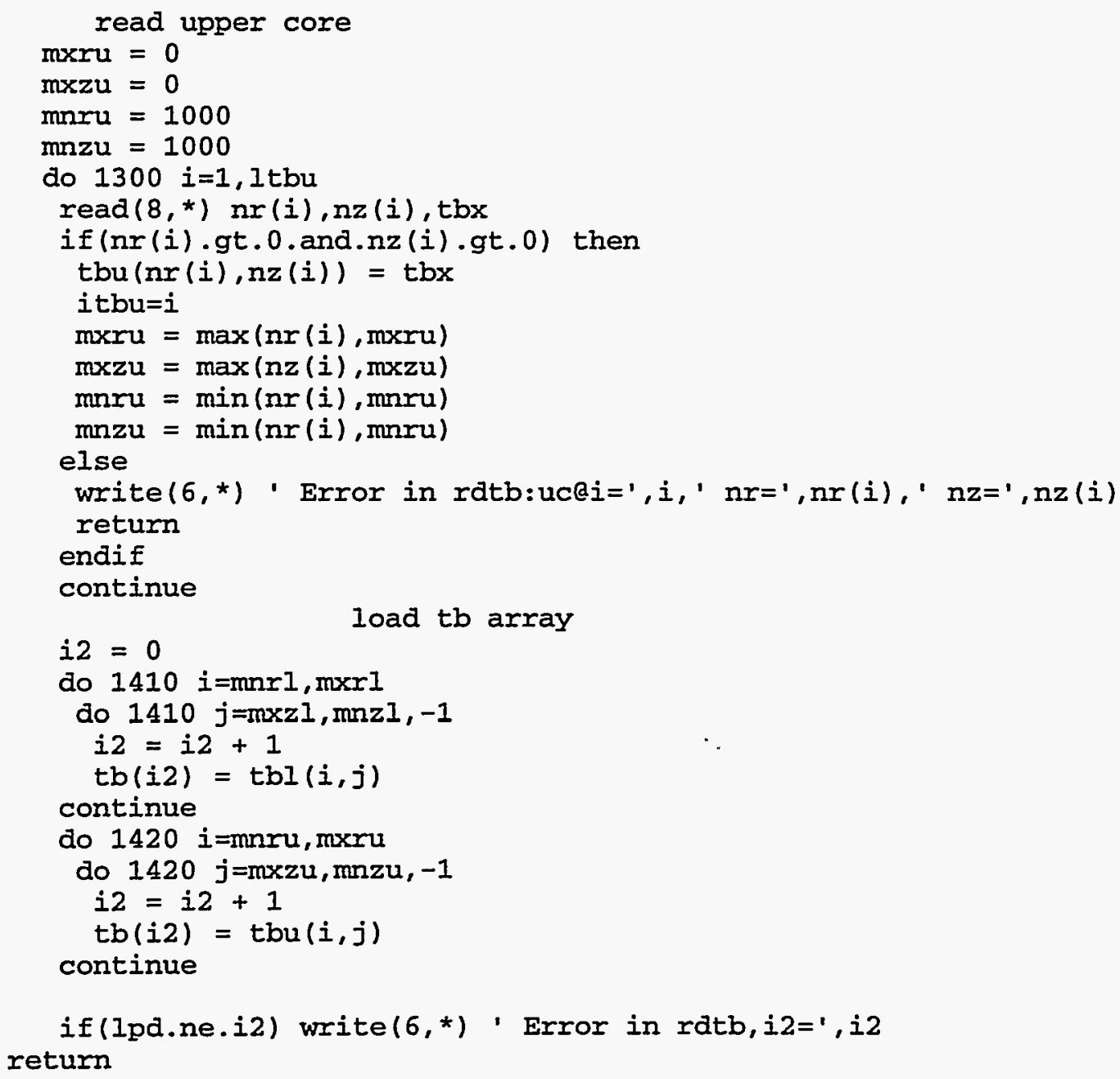




\section{A.14 \\ Table A.7. Code listing of RD-ALL (continued)}

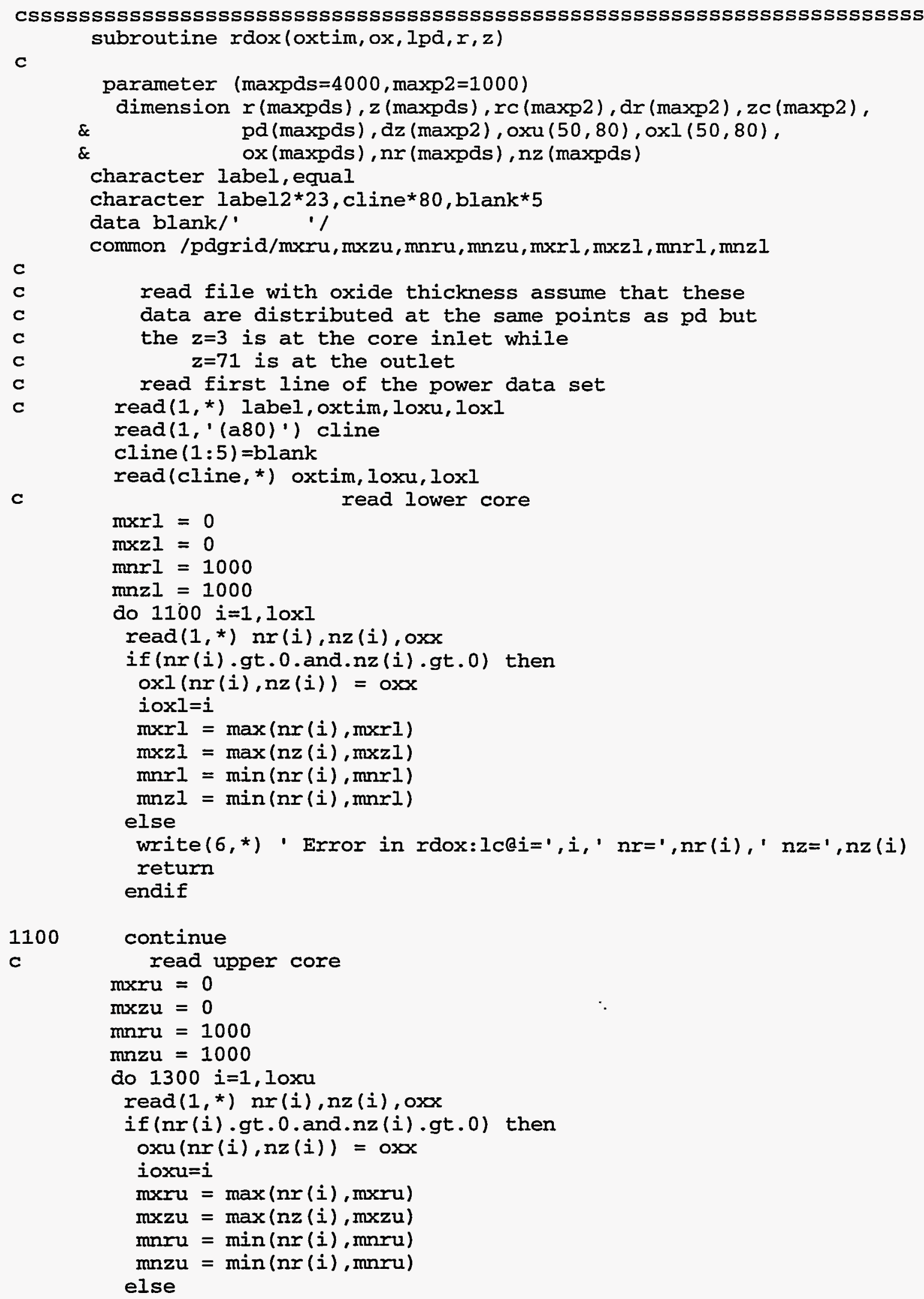




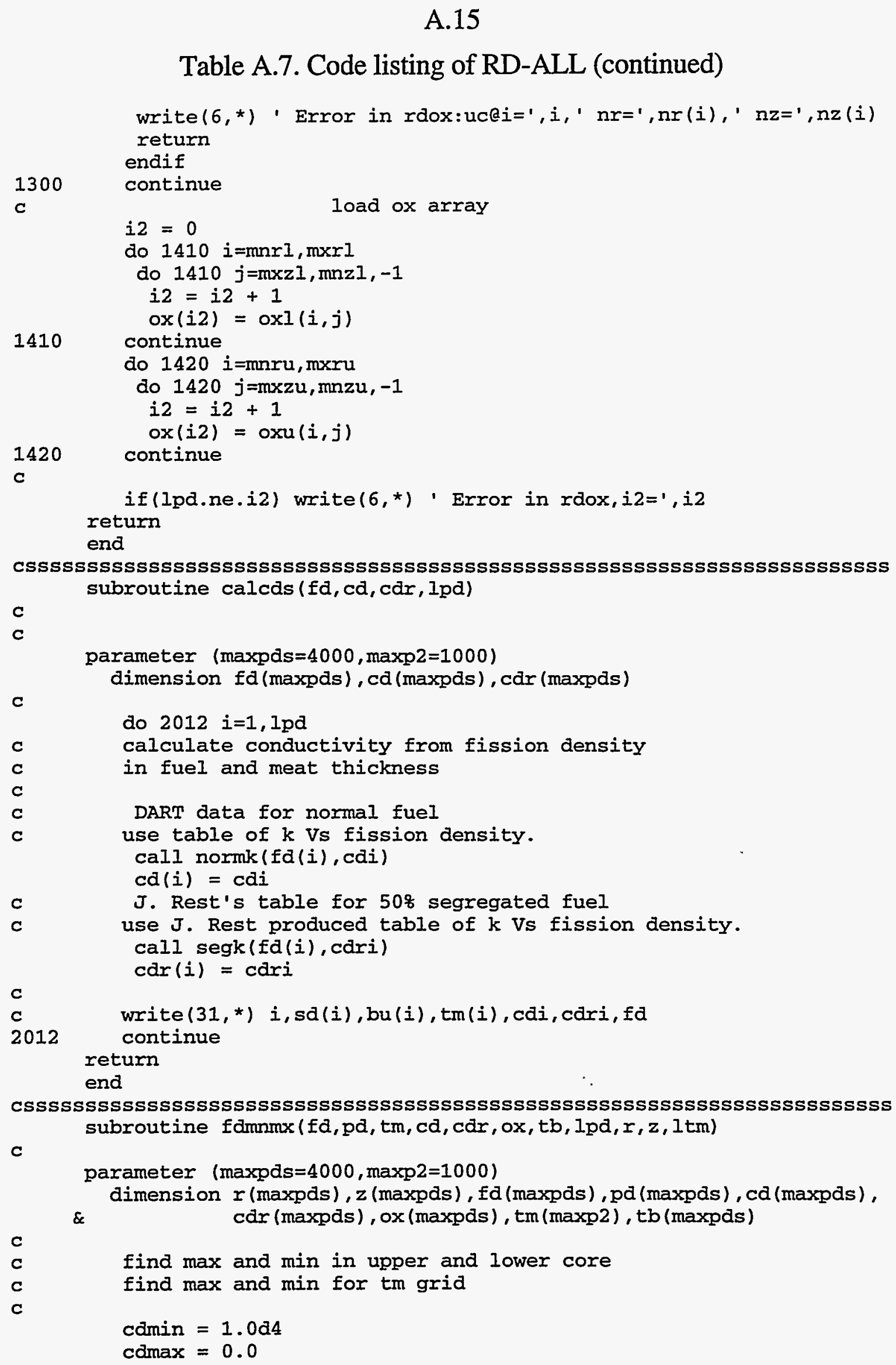




\section{A. 16}

Table A.7. Code listing of RD-ALL (continued)

c

c

c

c

C

c

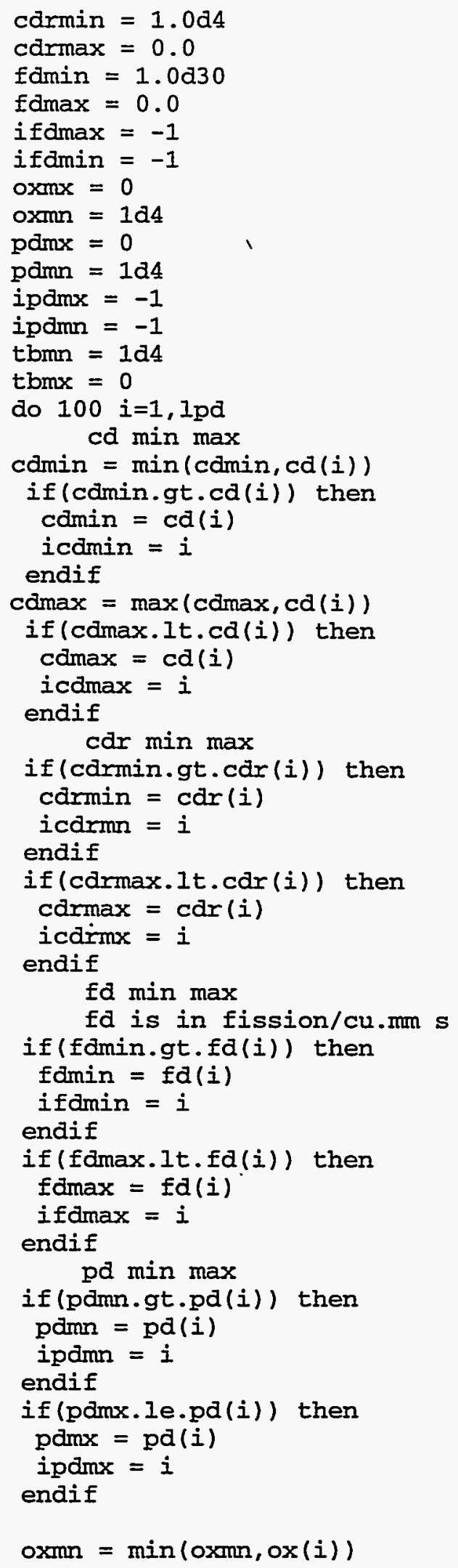




\section{Table A.7. Code listing of RD-ALL (continued)}

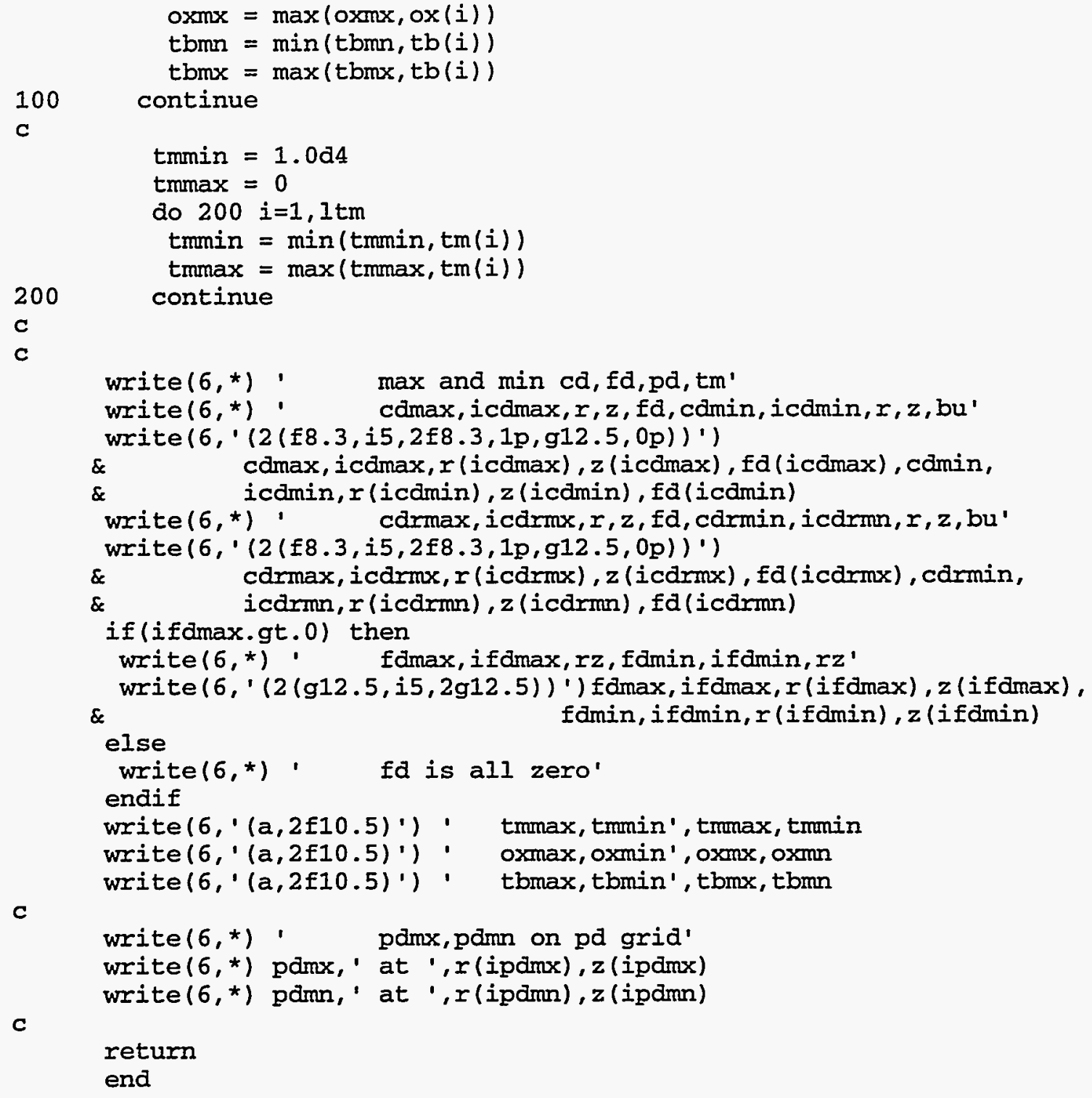

c

C 


\section{A.18}

\section{Table A.7. Code listing of RD-ALL (continued)}

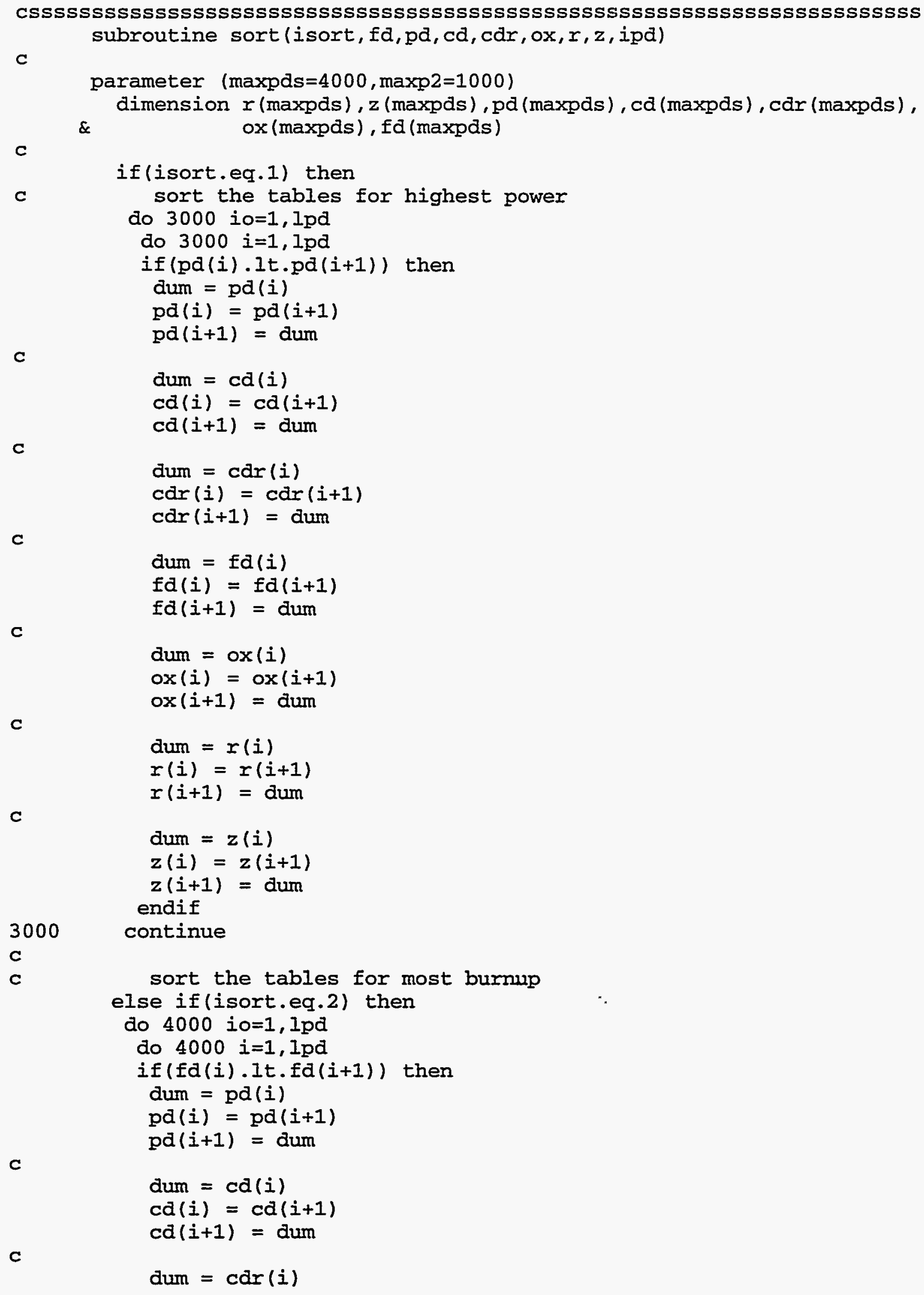

C

dum $=\operatorname{cdr}(i)$

C

dum $=f d(i)$

$f d(i)=f d(i+1)$

$f d(i+1)=d u m$

C

dum $=o x(i)$

$o x(i)=o x(i+1)$

ox $(i+1)=$ dum

c

dum $=r(i)$

$r(i)=r(i+1)$

$r(i+1)=$ dum

c

dum $=z(i)$

$z(i)=z(i+1)$

$z(i+1)=$ dum

3000 continue

c

sort the tables for most burmup

else if(isort.eq.2) then

do 4000 io $=1$, Ipd

do $4000 i=1$, Ipd

if(fd(i).It.fd(i+1)) then

dum $=p d(i)$

$p d(i)=p d(i+1)$

$\operatorname{pd}(i+1)=$ dum

C

dum $=c d(i)$

$c d(i)=c d(i+1)$

$c d(i+1)=d u m$

c

$\operatorname{dum}=\operatorname{cdr}(i)$

c 
A. 19

Table A.7. Code listing of RD-ALL (continued)

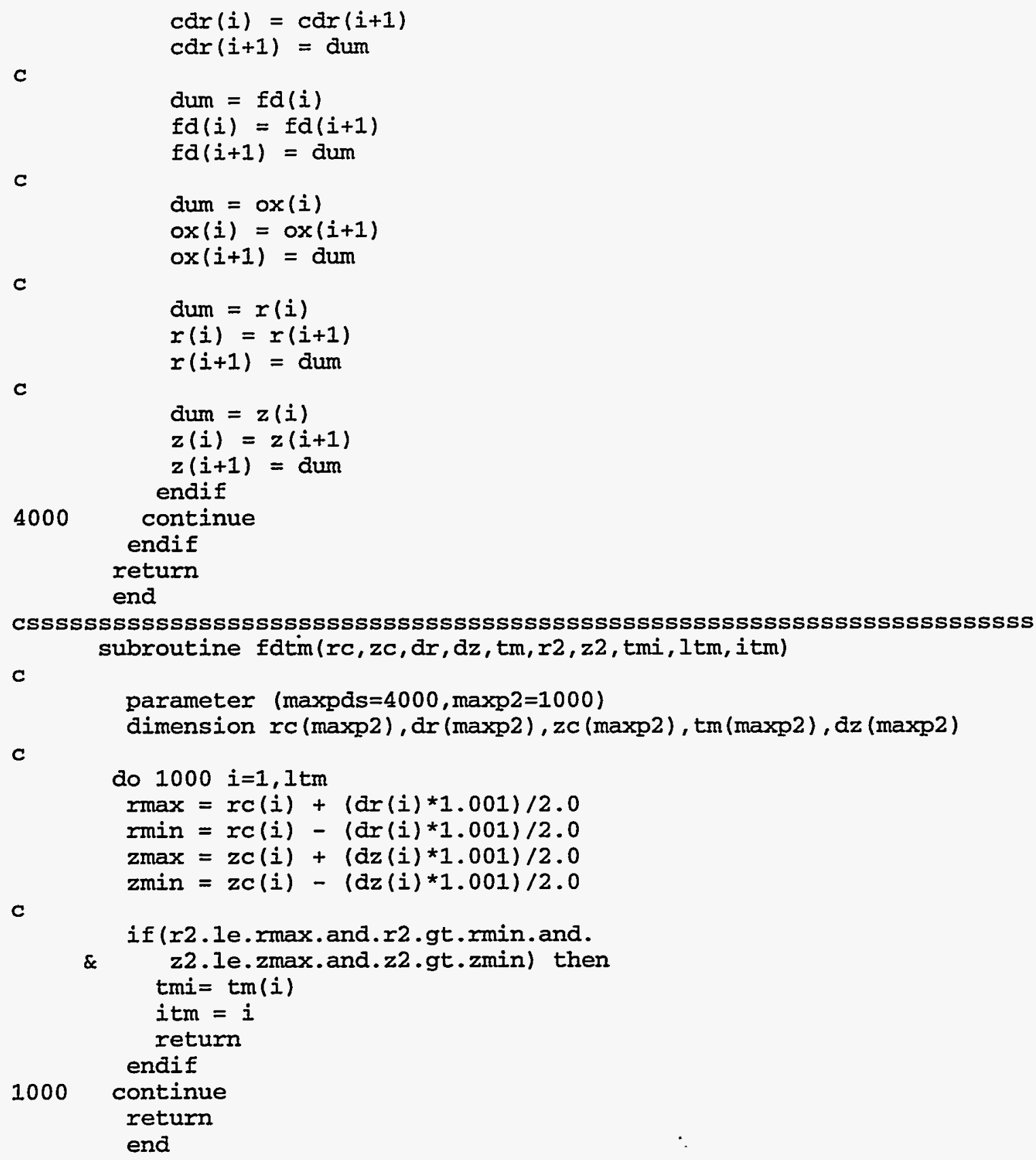

c

parameter (maxpds $=4000, \operatorname{maxp} 2=1000)$

dimension $r c(\operatorname{maxp} 2), d r(\operatorname{maxp} 2), z c(\operatorname{maxp} 2), t m(\operatorname{maxp} 2), d z(\operatorname{maxp} 2)$

c

c

\& z2.1e.zmax.and.z2.gt.zmin) then $\operatorname{tmi}=\operatorname{tm}(i)$

itm $=i$ return

endif

1000 continue

return

end 


\section{A.4 DRIVER PROGRAM RH7-ANSR}

The program RH7-ANSR reads the file tecconl and generates the input file necessary to run HEATING with user-modifications at each data point on the lower fuel plate for each time step. It repeats this process with the file tecconu.

The program is basically a set of write statements that output each line of a HEATING input file. Most of the data for the input lines are fixed. These are coded into the format statements as character strings. On some write statements a few variables are used to allow modifications of the input file consistent with the changed input parameters. The values of these variables are determined by the FORTRAN coding from the input parameters. Thus the program can adjust the model within the constraints of the generic model to perform an analysis using the local conditions.

For example, the local meat thickness changes the size and location of the region describing the segregated and normal fuel meat within the model. It also changes the size and location of the filler regions, the location of the nonbond void and the grid spacing definitions. The local relative power density with the local meat thickness and uncertainty factors determines the local heat generation. The local fission density determines the local thermal conductivity of normal and segregated fuel. The local coolant temperature determines the temperature of the convective boundary condition and the tabular function that describes the temperature dependent heat transfer coefficient.

Although this process is relatively straightforward, there are several situations that complicate the coding. One problem is that the numbers that define the region boundaries must be printed in the region data block of the input file to the same precision as in the grid data blocks. If the sequence of digits that describe a boundary of one region is not exactly the same as those for a boundary that should be adjacent, HEATING may not recognize that the regions should be touching. HEATING assumes that any portions of the modeling region that are not described by region data are volumes with adiabatic boundaries. In addition, HEATING will halt if it cannot match the region boundaries with the gross grid lines in the grid data blocks.

The region boundaries and gross grid lines are determined by arithmetic within the program. Roundoff to the output precision can produce values that are incorrectly identical in the output. This may produce region descriptions that are incorrect or in error. HEAT.ING will halt if it finds regions that shink to zero length in any dimension. Identical gross grids are also an unacceptable condition. It is also possible to calculate values that are not consistent with other fixed input. This situation can produce incorrect region boundaries or gross grid lines. For instance, if the minimum region 
boundary is larger than the maximum along one coordinate axis the code will stop. The gross grid lines must also be in increasing order.

For the region and grid data blocks, the logic must account for the possibility of regions that disappear. To prevent abnormal termination, these regions must be removed from the input. The logic must also prevent holes from being formed by adjacent regions that do not touch. The gross grid lines must be in ascending order with no duplicates.

The HEATING input technique of overlaying regions is used in RH7-ANSR to minimize some of these problem. It is possible to define a HEATING model where region definitions overlap one another. The code uses the order of region definitions to determine the properties of a node in the intersection. This technique reduces the required number of region definitions and allows several regions to be defined by fixed input, thus reducing the potential for errors in the coding and the probability of adjacent regions not touching.

In order to produce a properly formatted file there are additional constraints on the lines that are generated must which be observed. HEATING requires that every line be less than 70 characters long. In the Tabular Data Block, the many input numbers may be required. The logic produce lines that contain the data to sufficient precision and less characters than the HEATING limit. Several tables in the input require multiple lines. HEATING requires that such lines have a continuation character (@) in the first character position. The logic must be adaptive enough to produce input line for tables of different lengths. In addition, all keywords are required to start in the first location of the line. All other lines may start anywhere before the end of the line.

This generic model is representative of the two-element-core designs and would require slight modification to allow the three-element-core design.

\section{A.5 CODE LISTING FOR RH7-ANSR}

The program RH7-ANSR is listed in Table A.8. It was developed to run on an IBM RISC 6000 using FORTRAN version xlf 2.3. This program calls a C routine, DATIM, that is used to get the date and time. DATIM.C is listed in Table A.9. This routine was furnished by K. W. Childs.

The RH7-ANSR program listed in the table as a typical version. This version is one of the latest $95 \%$ limits case using the model that converts the segregated fuel material into $\mathrm{U}(\mathrm{Al}, \mathrm{Si})_{3}$ when the temperature exceeds the transition temperature. The fuel is enriched to $93 \%$. Other versions were 


\section{A. 22}

developed to use medium- and low-enriched fuel, to investigate various inspection requirements and to perform a nominal analysis.

A major variant of this program was developed to calculate the oxide growth based on the maximum heat flux in the HEATING model rather than use the TASHA results. This modification required two HEATING executions at every point for every time step. The first HEATING execution produced the heat flux estimate using the oxide thickness from the previous time step. An estimate of the oxide growth was made using the heat flux and temperatures. The second HEATING execution used the beginning of time-step parameters with this end-of-time-step estimated oxide thickness and recalculated the maximum temperatures and peaking factors for the time step. This method is similar to the TASHA technique and was developed for comparison. As mentioned in Sect. 4, this approach may not be strictly conservative. 


\section{A.23}

\section{Table A.8. Code listing of RH7-ANSR}

C

C

1

C

c

C

this code reads the $r, z$, pd, $c d, t m$, and ox data set and passes each data point to mk1-h7in which writes out a heating 7.2 input deck for this point. the heating 7.2 executable is then run. The hpf data is written to io from the executable (usrprt) this data is extracted and appended to the hpf data set. The print and plot data sets are then deleted. modified to read tb from teccon files

parameter (maxpds $=2000$ )

dimension rualmx (2, maxpds), dzual (2, maxpds), time (50)

character ctime*8, char $(15) * 1$

character card $* 160$, erstrt $* 5$, errlst $* 8$, errtt $1 * 160$, commd ${ }^{*} 160$, blk $* 160$

character pname $\star 80$, fname $\star 80$, tname $\star 80$, abuf $\star 20$, waltim $* 20$

data inh7/7/

data $t s h / 150.0 /, t s c / 100.0 /, t m a x o / 700.0 /$

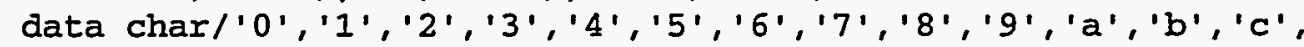
$\&$ 'd', 'e'/

data tstsmax $/ 0 /, t \operatorname{spfmx} / 0 /, t \operatorname{shfmx} / 0 /, t \operatorname{spmax} / 0 /, t s o m a x / 0 /$,

$\&$ $\operatorname{tsormx} / 0 /, \operatorname{tscdmx} / 0 /, \operatorname{tscdrmx} / 0 /, \operatorname{tsfdmx} / 0 /, \operatorname{txmax} / 0 /$

call datim(waltim)

write (abuf, '(a19)') waltim

read (abuf,' $(11 x, i 2,1 x, i 2,1 x, i 2)$ ') ih, im, is

itold $=$ is $+i m * 60+i h * 3600$

do $1 i=1,160$

$\operatorname{bIk}(i: i)=$ ' '

commnd $=\mathrm{blk}$

errttl $=\mathrm{blk}$

call system( 'date')

call system( 'uname $-a^{\prime}$ )

call system(

\&'cp /u/geg/anal/a/fy94/burnup/source/h7.2ans6/tmp') copy data files to /tmp

95\% limit data

pname $=1 / \mathrm{u} /$ geg $/$ anal $/ \mathrm{a} /$ fy $95 /$ tables $/ \mathrm{g} 693 / \mathrm{k}-573 \mathrm{k}$

no uncertainty data

pname $=$ '/home/geg/anal/a/fy94/tables/g693/G6-best $/$ ta-ox3 $/$ '

lpname $=$ lnblnk (pname)

tname $=1 / \mathrm{tmp} / \cdot$

Itname $=$ Inblnk (tname)

commnd $=$ ' $\mathrm{cp}$ '//pname $(1: 1$ pname $) / /$ teccon [ul] /tmp'

call system (comnin)

commind $=\mathrm{blk}$

call system( 'rm hpf')

call system( 'rm CPU')

write out header for hpf file commnd=

\& ' echo "variables $=r$ z pd c tm o p tx $f$ tb cr svt uvt tId" $>$ hpf " call system (commnd)

commind $=\mathrm{blk}$ 


\section{Table A.8. Code listing of RH7-ANSR (continued)}

C

C

c

10

c

11

c

c

C

c

21

c

30

c

c

$\mathrm{C}$

c

c

10

do 1000 icore $=1,2$

open normal and segregated fuel conductivity data sets if (icore.eq.1) fname = tname (1:1tname)//'tecconl' if (icore.eq.2) fname = tname (1:1tname)//'tecconu' open (unit=5, file=fname, status='old')

$$
\begin{aligned}
& \text { do } 10 \text { idum }=1,2 \\
& \text { read }\left(5, '(2160)^{\prime}\right) \text { card }
\end{aligned}
$$

do 11 idum=1, maxpds

rualmx (icore, $i d u m)=0$

dzual (icore, $i$ dum) $=0$

$$
\text { continue }
$$

izone $=0$

$i=0$

do 100 icn $=1,10000$

$i=i+1$

read unmodified teccon[lu] files

check for zone cards in data set

read $(5, '(a 160)$ ', end=101) card

if zone card then write out to hpf data set

if $(\operatorname{card}(1: 4)$.eq.'zone') then

izone $=$ izone +1

$i=1$

ctime $=\operatorname{card}(9: 18)$

do 21 icc $=80,1,-1$

if (card (icc:icc).ne.' ') then

iclast $=i c c+1$

go to 30

endif

continue

output last time step maximum and clear

if(izone.gt.1) call outmax(ctime,tstsmax, tspfmx, tshfmx,

$\&$

$\&$

$\&$

commnd = 'echo $\cdot$ ' // $/ \operatorname{card}(1:$ iclast $) / /$ ' ' > hpf'

call system(commind)

commnd $=\mathrm{blk}$

read $(5, \cdot(a 160)$ ', end $=101)$ card

if (card ( $1: 4)$.eq. 'zone')

$\varepsilon$

write $(6, *)$ ' Error two zones cards in a row'

endif

endif on zone

read $(\operatorname{card}, *)$ rc, zc, pd, cd, cdr, fd, tm, ox, tb

if (izone.eq.1) ox $=0$

delete heating input file before building the next case call system ('rm input')

open (unit=inh7, file=' input' , status='new')

delete all heating print files

call system ('rm print*')

delete all heating plot files

call system(' $\mathrm{rm}$ plot*') 


\section{Table A.8. Code listing of RH7-ANSR (continued)}

$\&$

$$
\text { close (inh7) }
$$

move plot data set to restart

call system('mv plot0000 restart')

run heating7 executable

call system('nice /tmp/h7.2ans6') extract hpf data and appended to hpf data set

commnd ='grep ",hpf" print*|sed -e "s/rzpcto,hpf,tx//" >> hpf' add fd tb and cdr to hpf data set

commnd ='grep ",hpf" print*|sed -e "s/rzpcto,hpf,tx//" > temphpf'

call system (commna)

commnd $=\mathrm{blk}$

open (unit=8, file= ' temphpf' , status='old')

rewind 8

read $(8, *) r 0, z 0, \operatorname{pd} 0, \mathrm{~cd} 0, \operatorname{tm} 0, \mathrm{ox} 0, \mathrm{pf0}, \mathrm{tx} 0$

if (tx0.gt.txmax) then

txmax $=t x 0$

commind $=\mathrm{blk}$

if (icore.eq.1) commnd = 'mv input inputl'//char (izone)

if(icore.eq.2) comma = 'mv input inputu'//char(izone)

call system (commnd)

endif

rewind 8

c

add seg \& ualx vol ave temp \& grab dzual\&rualmx to hpf data set call system ('rm temp')

commnd ='grep "volave" $\mathrm{pr}^{*} \mid \mathrm{sed}$-e "s/ volave .*=//">temp'

call system (commnd)

commin $=\mathrm{blk}$

open (unit $=8$, file=' temp' , status= ' old')

rewind 8

read $\left(8,{ }^{*}\right)$ segvat, ualvat, dzual (icore, $\left.i\right)$, rualmx(icore, $i$ )

read $(8, *)$ segvat, ualvat, dzu, rumx

dzual $(i c o r e, i)=\max (d z u a l(i c o r e, i), d z u)$

rualmx $(i c o r e, i)=\max ($ rualmx $(i c o r e, i)$, rumx $)$

close (8) add $T$ 1ds to hpf data set

call system('rm temp') still in mod - $12 / 17$

commnd ='grep "1dflux" pr*|sed -e "s/^ *1dflux .* $=/ /$ ">temp"

call system (commnd)

commnd $=\mathrm{blk}$

open (unit $=8$, file=' temp', status $=$ 'old')

rewind 8

$\operatorname{read}(8, *) \mathrm{d} 1, \operatorname{ts} 1 d, \operatorname{tmx} 1 d$

close ( 8 )

call system ('rm temphpf')

open (unit =8, file= ' temphpf', status= 'new')

write (8,

$\cdot(2(\mathrm{f} 6.1,1 \mathrm{x}), 3(\mathrm{f} 6.4,1 \mathrm{x}), 1 \mathrm{p}, 4(\mathrm{~g} 12.5,1 \mathrm{x}), 0 \mathrm{p}, \mathrm{f6} .2,1 \mathrm{x}, \mathrm{f6.4,1p}$,

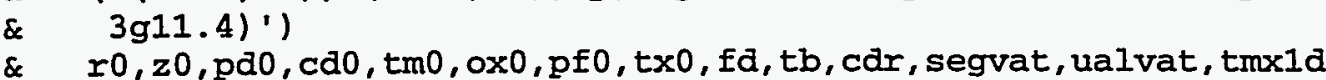
close (8)

call system('cat temphpf $\gg$ hpf') 


\section{A. 26 \\ Table A.8. Code listing of RH7-ANSR (continued)}

c

call datim(waltim)

write (abuf,'(a19)') waltim

read (abuf, ' $(11 x$, i2 $, 1 x, i 2,1 x, i 2)$ ') ih, im, is

itnew $=$ is $+i m * 60+i h * 3600$

if (itnew.ge.itold) then

itdif = itnew - itold

itold = itnew

else

itdif $=$ itnew $+(86400-$ itold $)$

itold = itnew

endif

call system('rm temptdif')

open (unit $=66$, file=' temptdif', status=' new' )

write $\left(66, '(i 8)^{\prime}\right)$ itdif

close (66)

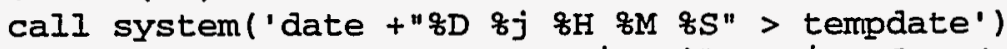

commind='grep "sed cp" pr*0* | tail $-1 \mid$ sed "s/ * E.* = *//" |

\& sed " $s /: / / g^{\prime}$ | paste tempdate temptdif - $>C P U$ '

call system (commnd)

C commnd $=\mathrm{blk}$

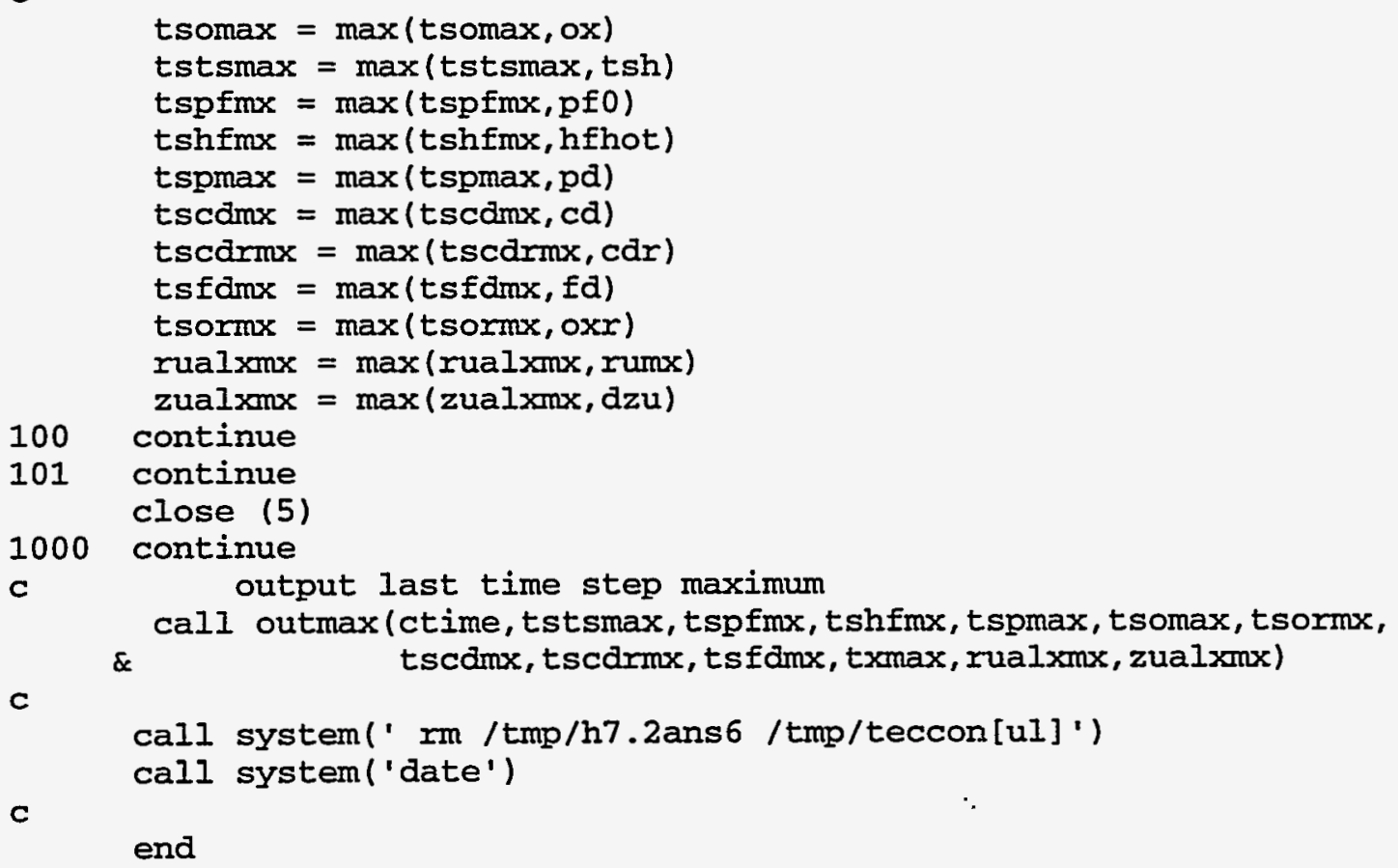




\section{A.27 \\ Table A.8. Code listing of RH7-ANSR (continued)}

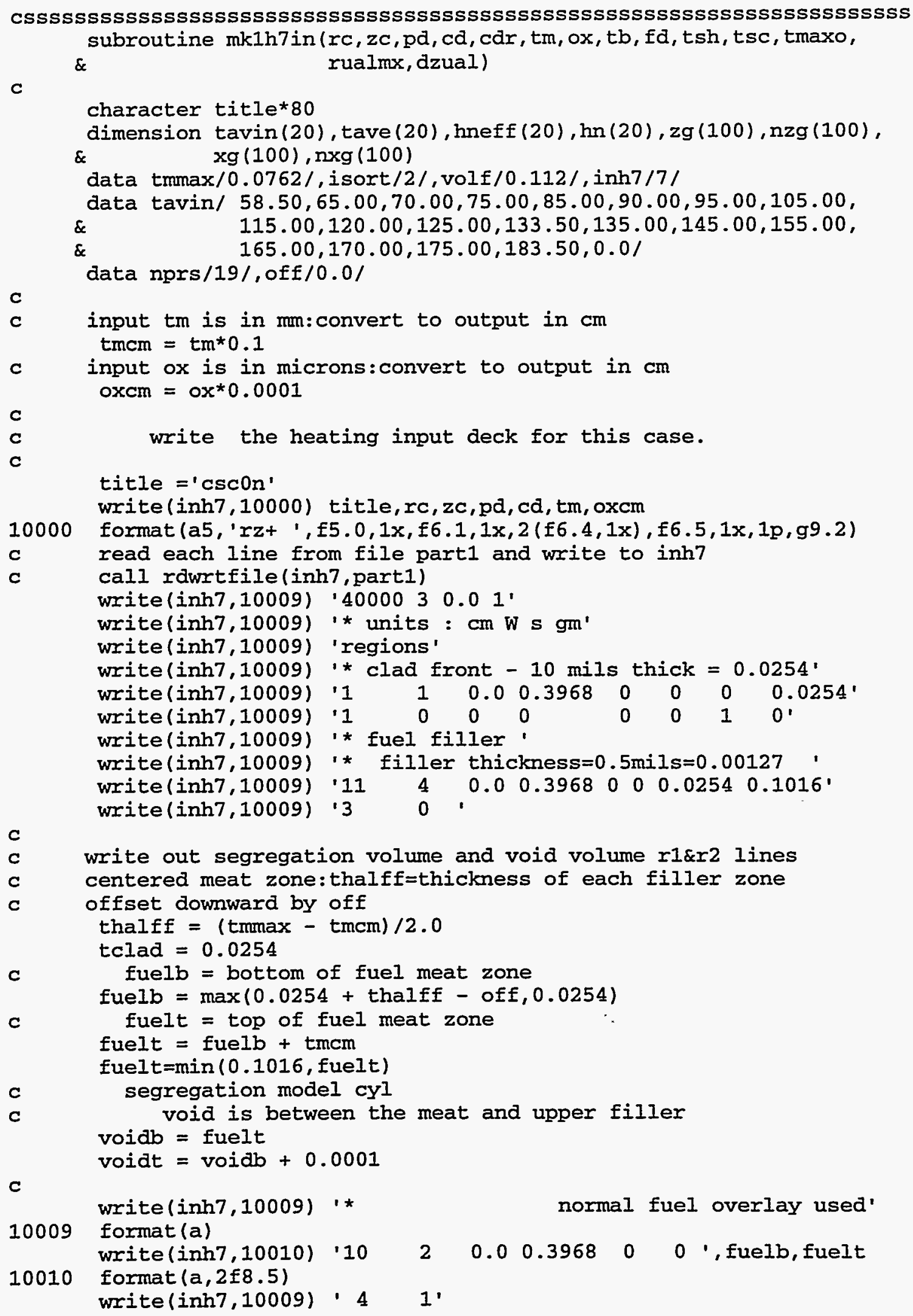


Table A.8. Code listing of RH7-ANSR (continued)

C

vof $=31.0$

segrad $=0.01552$

write(inh7,10021) ' * cyl seg.fuel overlay used, vof=', vof

10021 format $(a, f 6.2)$

write(inh7,10029) '20 30.0 ', segrad,' $0 \quad 0$ ', fuelb, fuelt

10029 format $(a, f 8.5, a, 2 £ 8.5)$

write (inh7,10009) ' 5 2'

C

cyl ualx overlay

if (rualmx.gt.1.0d-8) then

rualx $=\min ($ rualmx, segrad)

write (inh7,10021) '* cyl ualx.fuel overlay used'

zualb = fuelt - dzual

zualb $=\max (z u a l b$, fuelb)

write (inh7,10029) ' $50 \quad 50.0$ ',rualx, ' 0 ', zualb, fuelt

write $($ inh 7,10009$)$ ' 5 2'

endif

C

c write out the rest of region cards

c

write(inh7,10009) '*clad back'

$\begin{array}{lllllllll}\text { write (inh7, 10009) } & ' 2 & 1 & 0.0 & 0.3968 & 0 & 0 & 0.1016 & 0.1270 ' \\ \text { write (inh7,10009) } & ' 2 & 0 & 0 & 0 & 0 & 0 & 0 & 1 .\end{array}$

write (inh7,10009) '*'

write(inh7,10009) '* one dim model - detacted'

write (inh7,10009) '* clad front - 10 mils thick $=0.0254$ '

$\begin{array}{lllllllll}\text { write (inh7,10009) ' } 31 & 1 & 0.4 & 0.41 & 0 & 0 & 0 & 0.0254\end{array}$

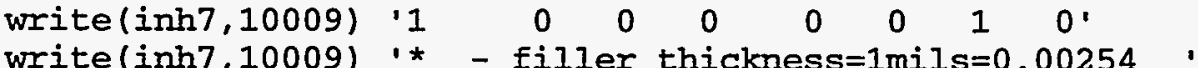

$\begin{array}{lllllllll}\text { write (inh7,10009) } & 34 & 4 & 0.4 & 0.41 & 0 & 0 & 0.0254 & 0.1016^{\prime}\end{array}$

write (inh7, 10009) ' 3 '0'

write (inh7,10009) ' *'

c

write out void here to be sure that all regions are overlaid write (inh7, 10009) '* '

write (inh7,10009) '* nonbond void $1 \mathrm{mmD}$ seg-fuel\&filler'

write (inh7,10011) ' 9000.00 .05000 , voidb, voidt

10011 format (a25, 2f8.5)

write $(\operatorname{inh} 7,10009) \cdot 0$ '

C

write (inh7,10009) '*

$\begin{array}{lllllll}\text { write }(\text { inh7,10010) } & 132 & 2 & 0.4 & 0.41 & 0 & 0\end{array}$

C

write(inh7,10010) '* clad back'

$\begin{array}{lllllllll}\text { write }(\operatorname{inh} 7,10010) & 133 & 1 & 0.4 & 0.41 & 0 & 0 & 0.1016 & 0.1270 \text { ' } \\ \text { write }(\operatorname{inh} 7,10010) & 12 & 0 & 0 & 0 & 0 & 0 & 0 & 11^{\prime}\end{array}$

write $(\operatorname{inh} 7,10010)$ '*'

C

write out the first of the materials cards

write (inh7, 10009) 'materials'

write(inh7,10009) ' $* * \star \star \star \star * \star * * \star$ clading aluminum 6061-to'

write (inh7,10009) ' 1 al6061-0 1 0 0 1'

write(inh7,10009) , $* \star \star \star \star \star * \star * \star *$ fuel filler al powder '

write(inh7,10009) ' 4 filler 1.7

write $(\operatorname{inh} 7,10009)$ 


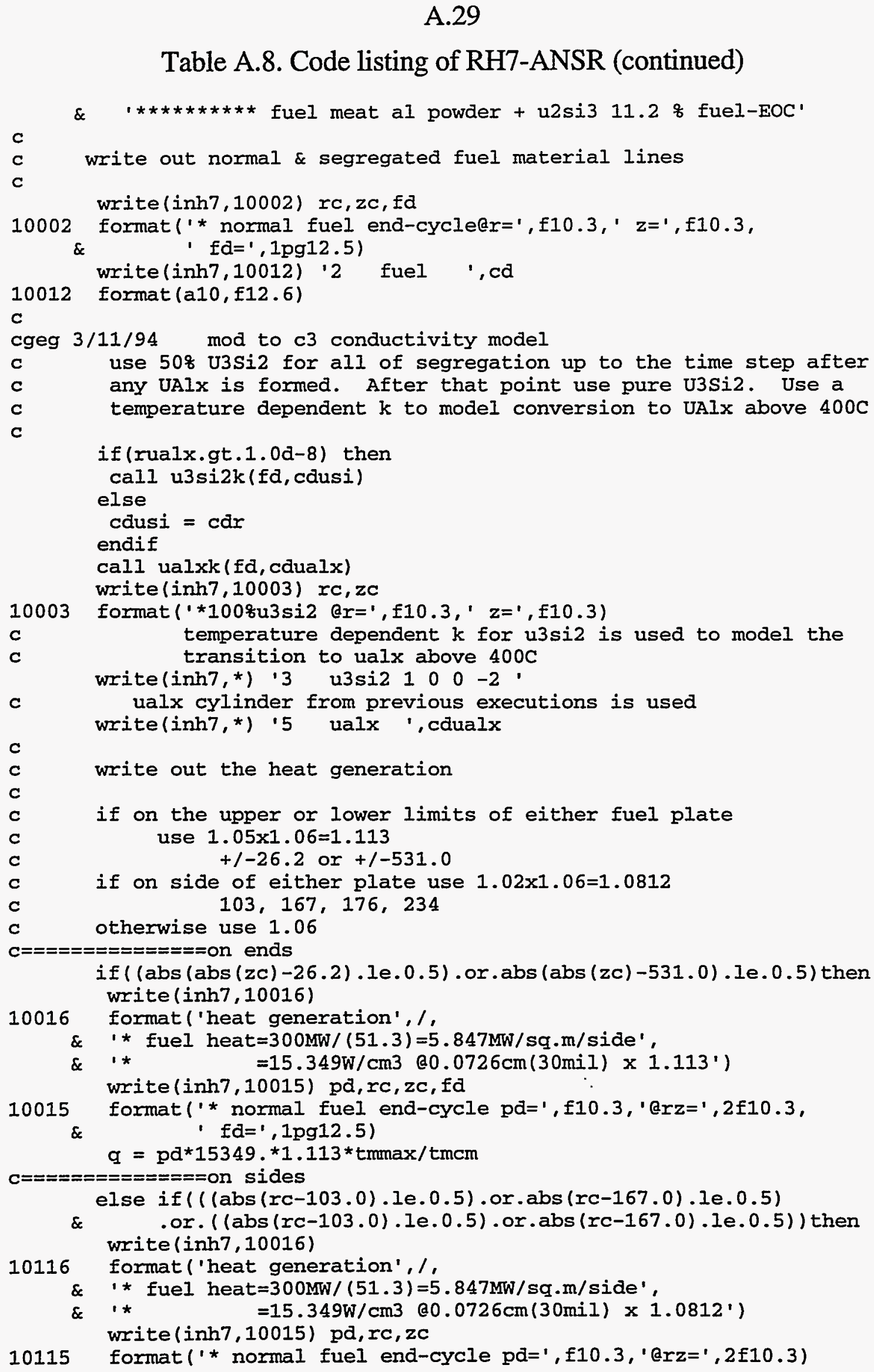




\section{A.30}

\section{Table A.8. Code listing of RH7-ANSR (continued)}

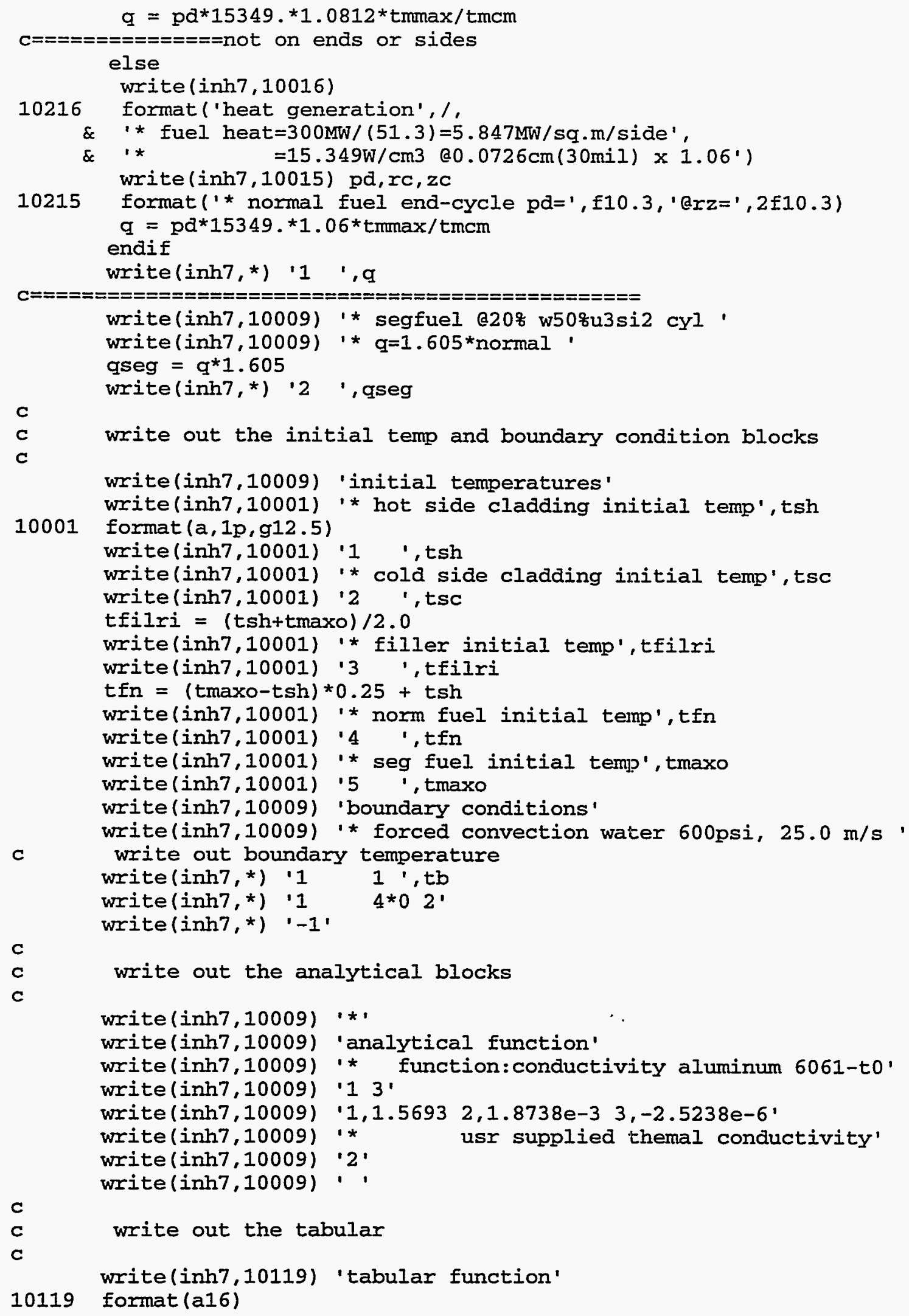




\section{A. 31 \\ Table A.8. Code listing of RH7-ANSR (continued)}

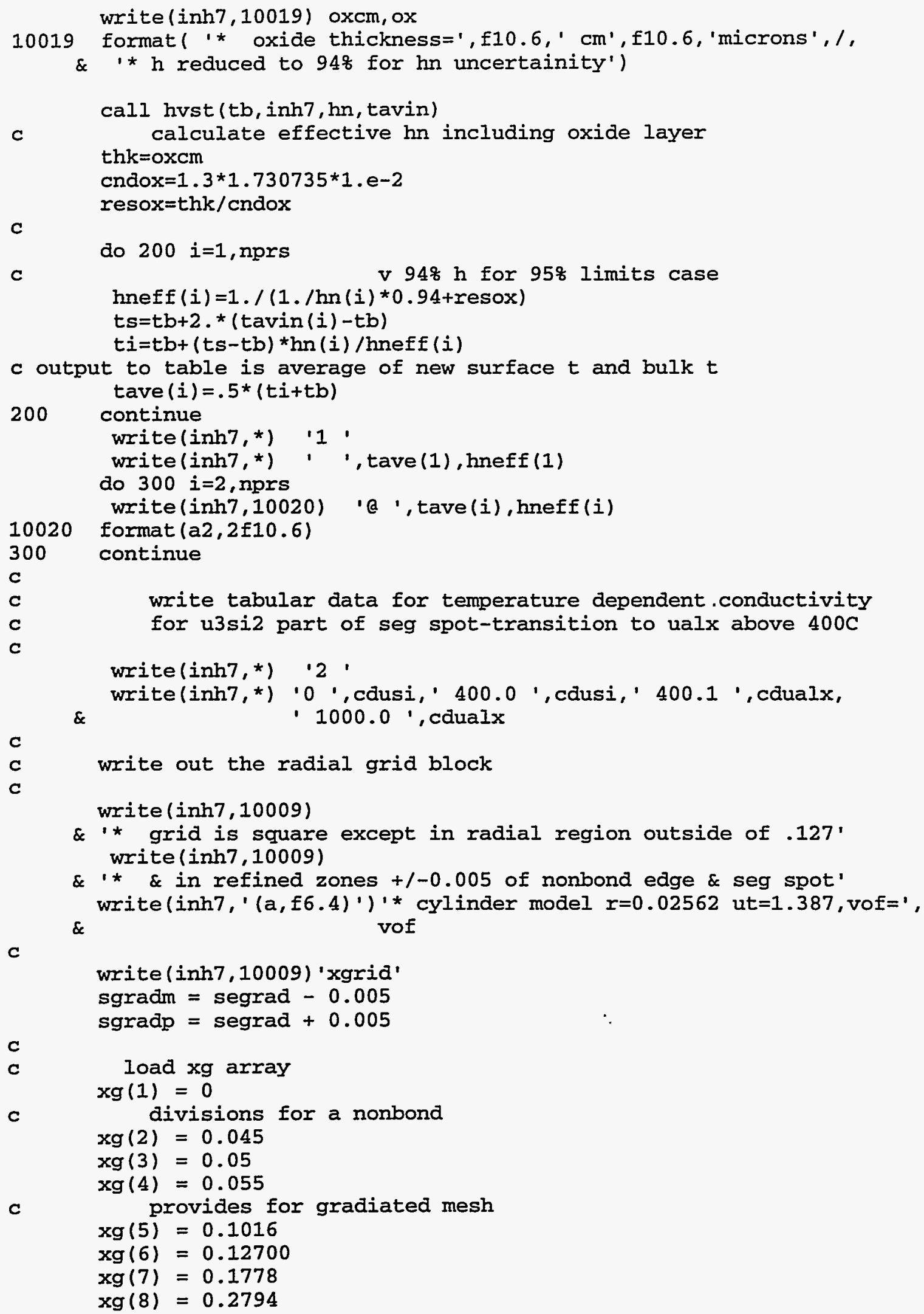

c provides for gradiated mesh

$\mathrm{xg}(5)=0.1016$

$\mathrm{xg}(6)=0.12700$

$x g(7)=0.1778$

$\mathrm{xg}(8)=0.2794$ 


\section{A.32}

Table A.8. Code listing of RH7-ANSR (continued)

c

C

C

c

C

C

C $\mathrm{xg}(9)=0.3968$

$1 d$ model

$\mathrm{xg}(10)=0.4$

$x g(11)=0.41$

segregation model

$\mathrm{xg}(12)=$ sgradm

$x g(13)=$ segrad

$\mathrm{xg}(14)=$ sgradp

the refined section inside the nonbond radius is the

last input

$i x g=14$

if (rualx.gt.1.0d-8) then

if (rualx.ge.sgradm.and.rualx.lt.segrad) then

$\mathrm{xg}(5)=$ rual $\mathrm{x}$

else if(rualx.1t.sgradm) then

$i x g=i x g+1$

$x g(i x g)=$ rualx

endif

endif

sort $x$ grid lines

call sortg $(x g, i x g)$

write out grid lines

if(ixg.le.7) then

write $(i n h 7,(7 f 8.5) ') \quad(x g(i w), i w=1, i x g)$

else

write (inh7, '(7f8.5)') (xg(iw), iw=1,7)

write (inh7,'('G, ', 7f8.5)') (xg(iw), iw=8, ixg)

endif

set divisions

dro $=\operatorname{sgradm} / 4$

$\operatorname{dr} 1=0.01 / 8$

$\mathrm{dr2}=(0.1016-0.05) / 9$

$\mathrm{dr} 3=\mathrm{dr} 2 * 2$

$\mathrm{dr} 4=\mathrm{dr} 3 * 2$

$\mathrm{dr} 5=\mathrm{dr} 4 * 2$

$\operatorname{dr} 6=\operatorname{dr} 5 * 2$

$n \times g(1)=\max (1, \operatorname{ifix}(x g(2) / \operatorname{ar} 0))$

do $310 i 1=2$, $i x g$

if ( $x g(i 1) .1 t . s g r a d m)$ then

$n \times g(i 1-1)=\max (1, i f i x((x g(i 1)-x g(i 1-1)) / \operatorname{ar} 0))$

else if(xg(i1).le.sgradp) then

$n \times g(i 1-1)=\max (1, \operatorname{ifix}(\operatorname{xg}(i 1)-x g(i 1-1)) / \operatorname{ar} 1))$

else if(xg(i1).1e.0.045) then

$\operatorname{nxg}(i 1-1)=\max (1, i f i x((x g(i 1)-x g(i 1-1)) / a r 2))$

else if(xg(i1).le.0.055) then

$\operatorname{nxg}(i 1-1)=\max (1, \operatorname{ifix}(\operatorname{xg}(i 1)-x g(i 1-1)) / \operatorname{ar} 1))$

else if(xg(i1).1e.0.1016) then

$\operatorname{nxg}(i 1-1)=\max (1, i f i x((x g(i 1)-x g(i 1-1)) / \operatorname{dr2}))$

else if $(x g(i 1) .1$ e.0.1270) then

$\operatorname{nxg}(i 1-1)=\max (1, \operatorname{ifix}((x g(i 1)-x g(i 1-1)) / a r 3))$

else if(xg(i1).le.0.1778) then

$n \times g(i 1-1)=\max (1, i f i x((x g(i 1)-x g(i 1-1)) / d r 4))$

else if(xg(i1).le.0.2794) then

$n \times g(i 1-1)=\max (1, i f i x((x g(i 1)-x g(i 1-1)) / \operatorname{dr} 5))$

else if(xg(i1).1e.0.3968) then

$\operatorname{nxg}(i 1-1)=\max (1, i f i x((x g(i 1)-x g(i 1-1)) / \operatorname{ar} 6))$ 
Table A.8. Code listing of RH7-ANSR (continued)

else

$\operatorname{nxg}(i 1-1)=1$

endif

continue

write(inh7, '(23i3)') (nxg(iw), iw=1, ixg-1)

C

c

write out the axial grid block

c

C

write(inh7,10009) '* axial grid adapted to seg fuel cyl' write(inh7, 10009) 'zgrid'

$\mathrm{zg}(1)=0$ load $\mathrm{zg}$ array

$\mathrm{zg}(2)=0.0254$

$\mathbf{z g}(3)=$ voidb

$\mathrm{zg}(4)=$ voidt

$\mathbf{z g}(5)=$ fuelb

$z g(6)=$ fuelt

$\mathrm{zg}(7)=0.1016$

$\mathrm{zg}(8)=0.1270$

$i z g=8$

if (rualx.gt.1.0d-8) then

$i z g=i z g+1$

zg(izg) $=$ zualb

endif

c sort the $z$ grid lines

call sortg(zg,izg)

c write out grid lines

if(izg.le.7) then

write(inh7,' (7f8.5)') (zg(iw), iw=1,izg)

else

write (inh7,' (7f8.5)') (zg(iw), iw=1,7)

write (inh7, (' $G$ ' ', 7f8.5)') (zg(iw), iw=8,izg)

endif

$c$

c set divisions

$\mathrm{dz}=0.0254 / 10$

$\operatorname{nzg}(1)=\max (1, \operatorname{ifix}(z g(2) / \mathrm{dz}))$

do $315 i 1=2$, $i z g$

315

$\mathrm{nzg}(\mathrm{i1}-1)=\max (1, \operatorname{ifix}((\mathrm{zg}(\mathrm{i1})-\mathrm{zg}(\mathrm{i1}-1)) / \mathrm{dz}))$

continue

write (inh7,' (23i3)') (nzg(iw), iw=1, izg-1)

c

write out the rest of the input deck

c

write (inh7, '(a)') ' * '

write(inh7,'(a)') 'steady state parameters'

write (inh7,' (a)') '2,100,1.0d-5'

write(inh7,'(a)') io '

C

return

end 


\section{A.34 \\ Table A.8. Code listing of RH7-ANSR (continued)}

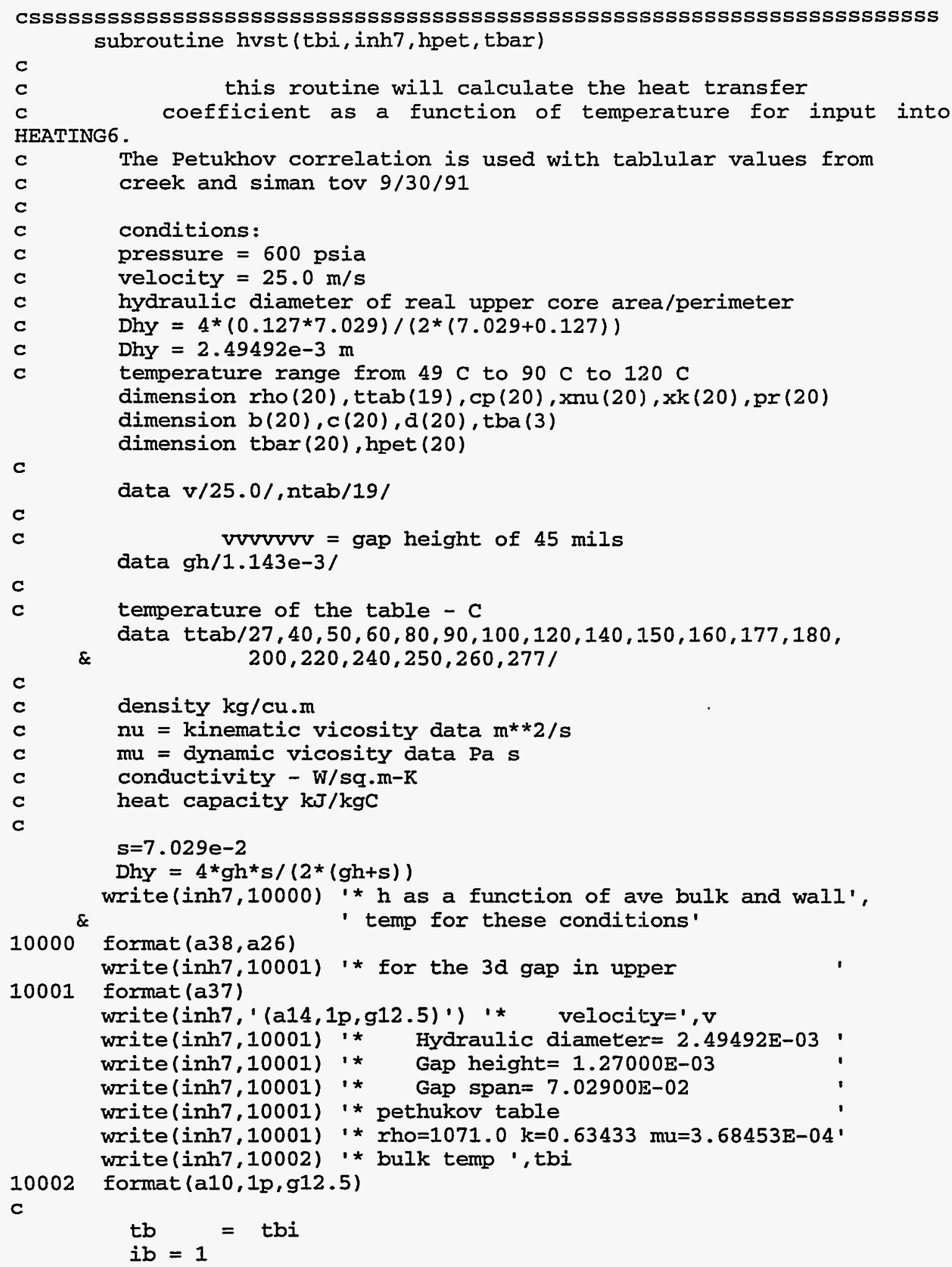

c

c at a bulk temperature of tb the heat transfer coefficient is a c function of the wall temperature using the petuhkov correlation. 


\section{A.35 \\ Table A.8. Code listing of RH7-ANSR (continued)}

C

C

C

C

C

C

c

c

C

C

C

c

c

$\&$

c

$\&$

c

c

c

c

10003

$\&$

for each temperature in the table find the heat transfer coefficient for the upper and lower core conditions.

find friction factor (Filonenko) for rectangular channel

ff $=(1.0875-0.1125(\mathrm{~b} / \mathrm{s})) *(1.82 * \mathrm{~d} \log 10(\mathrm{reb})-1.64) * *-2$

reb $=$ Bulk Reynolds number $-\mathrm{V} *$ Dhy/xnub

xnub = relative viscosity at tb

find the kinematic viscosity at tb

density of $\mathrm{D} 20$

$t f=1.8 * t b+32$.

rho(ib) $=1117.772605-0.077855 * t f-8.42 e-4 * t f * \star 2$

conductivity of $\mathrm{D} 20$

$t 1=(1.8 * t b+491.67) / 1 \mathrm{e} 4$

$x k(i b)=-0.4521496+36.0743280 * t 1-357.99773221 * t 1 * \star 2$

$+924.0219962 * t 1 * * 3$

specific heat of $\mathrm{D} 2 \mathrm{O}$

$c p(i b)=2.237124+122.217151 * t 1-2303.384060 * t 1 * * 2$

$+13555.737878 * t 1 * * 3$

dynamic viscosity of $\mathrm{D} 20$

$x m u b=-1.111606 e-4+9.46 e-8 * t f+0.0873655375 / t E+$

$0.4111103409 / \mathrm{t} \pm * * 2$

kinematic viscosity of $\mathrm{D} 2 \mathrm{O}$

$\operatorname{xnu}(i b)=x m u b /$ rho $(i b)$

reb $=V^{*}$ Dhy/xnu(ib)

ff $\quad=(1.0875-0.1125 *(\mathrm{gh} / \mathrm{s})) /(1.82 * \log 10($ reb $)-1.64) * \star 2$

calculate bulk Prandtl number and viscosity $p r(i b)=1000.0 * x n u(i b) * r h o(i b) * c p(i b) / x k(i b)$

write(inh7,10003) '*This gives $x$ mub $=$ ', xnu(ib), ' Prb= ',pr(ib)

format $(a 18,1 \mathrm{p}, \mathrm{g} 12,5, \mathrm{a} 6, \mathrm{~g} 12.5)$

write (inh7, 10004) '*This gives Reb= ',reb,' $f f=$ ',ff, ' upper'

10004 format $(a 17,1 p, g 12.5, a 5, g 12.5, a 9)$

find Nusselt number at each wall temperatures.

do $200 i=1$, ntab

find the fluid conductivity at the wall (tw=ttab(i))

tw $\quad=\operatorname{tab}(i)$

cal the fluid absolute viscosity at the wall viscosity of D2O

$t f=1.8 * t w+32$.

xmuw $=-1.111606 e-4+9.46 e-8 * t f+0.0873655375 / t f+$

$\&$

$0.4111103409 / \mathrm{t} \pm * 2$

rhow $=1117.772605-0.077855 * t f-8.42 e-4 * t f * \star 2$

xnuw $=$ xmuw $/$ rhow

$c$

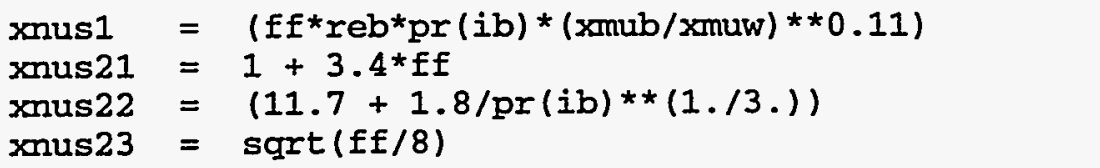




\section{A.36}

\section{Table A.8. Code listing of RH7-ANSR. (continued)}

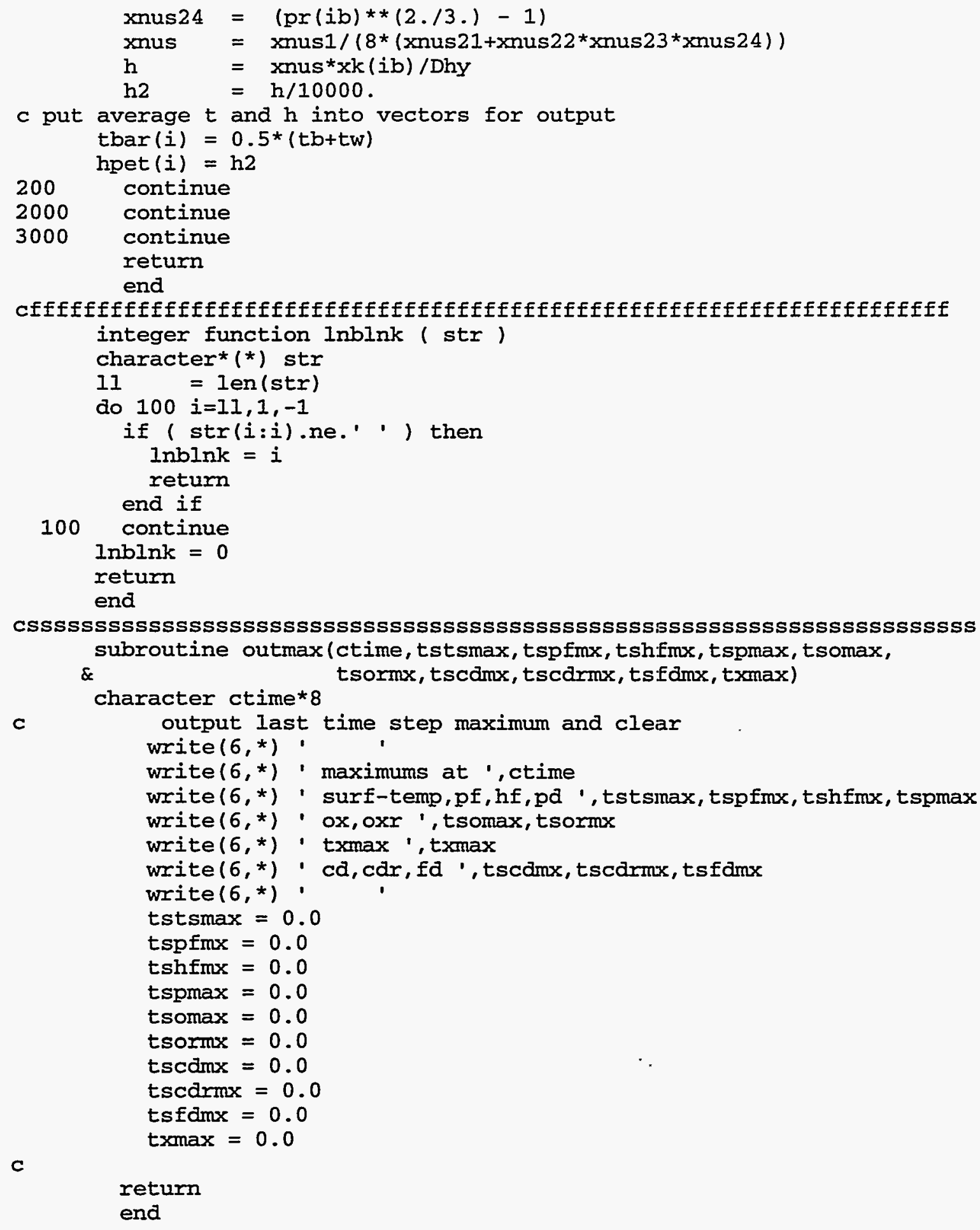




\title{
A.37 \\ Table A.8. Code listing of RH7-ANSR (continued)
}

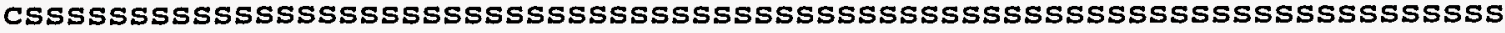 \\ subroutine u3si2k (fd, cdi)
}

C

C

C

C

C

C

C

c

if(Ed.1t.0.0) then

write $\left(6,{ }^{*}\right)$ ' error in table read $f d<0$ '

stop

endif

C

imatch $=0$

do $100 i=1$, ifdlast

if(fa.ge.fatab(i)) imatch $=i$

100 continue

if(fd.ge.fatab(ifdlast)) then

$\mathrm{fd}$ is fission density in fission / cu.cm s

tab is conductivity $\mathrm{w} / \mathrm{mK}$

dimension catab(81), fdtab (81)

fdtab fission density per cu.cm e+21/ 3.30521

for segregated fuel ( $50 \%$ u3Si2) j.rest data

data fdtab/0, 1.0010E+20,2.0020E+20,3.0030E+20,4.0040E+20,

\& $5.0050 \mathrm{E}+20,6.0060 \mathrm{E}+20,7.0070 \mathrm{E}+20,8.0081 \mathrm{E}+20,9.0091 \mathrm{E}+20$,

$1.0010 \mathrm{E}+21 ， 1.1011 \mathrm{E}+21 ， 1.2012 \mathrm{E}+21 ， 1.3013 \mathrm{E}+21 ， 1.4014 \mathrm{E}+21$,

$1.5015 \mathrm{E}+21 ， 1.6016 \mathrm{E}+21 ， 1.7017 \mathrm{E}+21 ， 1.8018 \mathrm{E}+21,1.9019 \mathrm{E}+21$,

2.1021E+21，2.2022E+21，2.3023E+21，2.4024E+21

3.1031E+21, 3.2032E+21，3.3033E+21，3.4034E+21

$4.0040 \mathrm{E}+21 ， 4.1041 \mathrm{E}+21 ， 4.2042 \mathrm{E}+21 ， 4.3043 \mathrm{E}+21 ， 4.4044 \mathrm{E}+21$,

年

7. $.5075 \mathrm{E}+21 ， 7.6076 \mathrm{E}+21 ， 7.7077 \mathrm{E}+21 ， 7.8078 \mathrm{E}+21 ， 7.9079 \mathrm{E}+21$, $1.4268 \mathrm{E}+01,1.4210 \mathrm{E}+01,1.4156 \mathrm{E}+01,1.4104 \mathrm{E}+01$ 1.4054E+01，1.4005E+01，1.3959E+01，1.3913E+01，1.3869E+01， $1.3826 \mathrm{E}+01,1.3784 \mathrm{E}+01,1.3742 \mathrm{E}+01,1.3702 \mathrm{E}+01,1.3663 \mathrm{E}+01$, 3624E+01，1.3585E+01，1.3528E+01，1.3470E+01，1.3415E+01, $1.3087 \mathrm{E}+01 ， 1.3032 \mathrm{E}+01,1.2978 \mathrm{E}+01,1.2923 \mathrm{E}+01,1.2869 \mathrm{E}+01$, 1.2814E+01，1.2760E+01，1.2706E+01，1.2651E+01，1.2598E+01， 2544E+01，1.2490E+01，1.2434E+01，1.2377E+01，1.2321E+01, 列 $1.1984 \mathrm{E}+01,1.1929 \mathrm{E}+01,1.1873 \mathrm{E}+01 ， 1.1818 \mathrm{E}+01,1.1764 \mathrm{E}+01$, $1.1709 \mathrm{E}+01,1.1655 \mathrm{E}+01,1.1601 \mathrm{E}+01,1.1548 \mathrm{E}+01,1.1494 \mathrm{E}+01$, $1441 \mathrm{E}+01,1.1389 \mathrm{E}+01,1.1336 \mathrm{E}+01,1.1284 \mathrm{E}+01,1.1233 \mathrm{E}+01$ 列 $+1.1131 \mathrm{E}+01,1.0005 \mathrm{E}+01,6.5921 \mathrm{E}+00,4.4809 \mathrm{E}+00$ $3.9516 \mathrm{E}+00,3.7352 \mathrm{E}+00,3.6367 \mathrm{E}+00,3.5776 \mathrm{E}+00,3.5317 \mathrm{E}+00$, $3.4893 \mathrm{E}+00,3.4461 \mathrm{E}+00,3.4011 \mathrm{E}+00,3.3540 \mathrm{E}+00,3.3052 \mathrm{E}+00$, 


\section{A.38 \\ Table A.8. Code listing of RH7-ANSR (continued)}

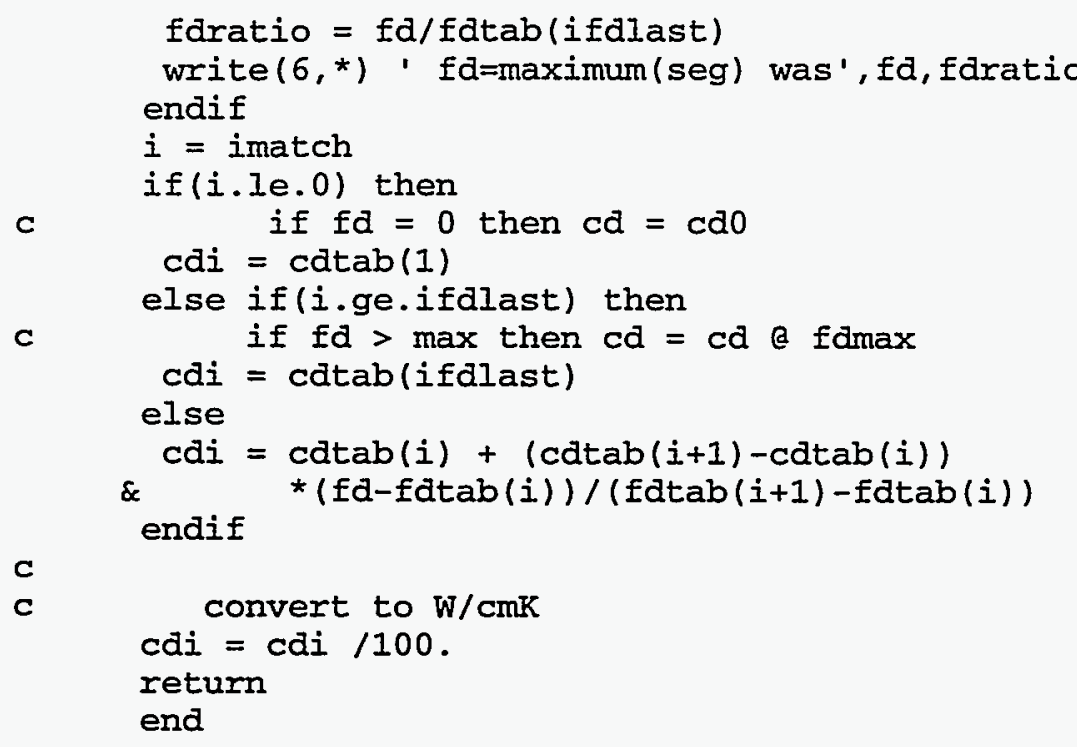

Cssssssssssssssssssssssssssssssssssssssssssssssssssssssssssssssss subroutine ualxk (fd,cdi)

C.

c $\quad \mathrm{fd}$ is fission density in fission / $\mathrm{cu} . \mathrm{cm} \mathrm{s}$

c for valx fuel j.rest data

c cdtab is conductivity $\mathrm{W} / \mathrm{mK}$

c cdi is conductivity $W / \mathrm{cmk}$

C

dimension cdtab(202), fdtab(202)

data ifdlast/202/

C

fdtab fission density per cu.cm

data fdtab/0, 1.0010E+20,2.0020E+20,3.0030E+20,4.0040E+20,

$\& \quad 5.0050 \mathrm{E}+20,6.0060 \mathrm{E}+20,7.0070 \mathrm{E}+20,8.0081 \mathrm{E}+20,9.0091 \mathrm{E}+20$,

$\& \quad 1.0010 \mathrm{E}+21,1.1011 \mathrm{E}+21,1.2012 \mathrm{E}+21,1.3013 \mathrm{E}+21,1.4014 \mathrm{E}+21$,

$\& \quad 1.5015 \mathrm{E}+21,1.6016 \mathrm{E}+21,1.7017 \mathrm{E}+21,1.8018 \mathrm{E}+21,1.9019 \mathrm{E}+21$,

$\& \quad 2.0020 E+21,2.1021 E+21,2.2022 E+21,2.3023 E+21,2.4024 E+21$,

$\& \quad 2.5025 \mathrm{E}+21,2.6026 \mathrm{E}+21,2.7027 \mathrm{E}+21,2.8028 \mathrm{E}+21,2.9029 \mathrm{E}+21$,

$\& \quad 3.0030 \mathrm{E}+21,3.1031 \mathrm{E}+21,3.2032 \mathrm{E}+21,3.3033 \mathrm{E}+21,3.4034 \mathrm{E}+21$,

$\& \quad 3.5035 E+21,3.6036 E+21,3.7037 E+21,3.8038 E+21,3.9039 E+21$,

\& $4.0040 E+21,4.1041 E+21,4.2042 E+21,4.3043 E+21,4.4044 E+21$,

$\& \quad 4.5045 \mathrm{E}+21,4.6046 \mathrm{E}+21 ， 4.7047 \mathrm{E}+21 ， 4.8048 \mathrm{E}+21 ， 4.9049 \mathrm{E}+21$,

$\& \quad 5.0050 \mathrm{E}+21 ， 5.1051 \mathrm{E}+21 ， 5.2052 \mathrm{E}+21 ， 5.3053 \mathrm{E}+21 ， 5.4054 \mathrm{E}+21$ ，

$\& \quad 5.5055 \mathrm{E}+21,5.6056 \mathrm{E}+21,5.7057 \mathrm{E}+21,5.8058 \mathrm{E}+21,5.9059 \mathrm{E}+21$,

$\& 6.0060 E+21 ， 6.1061 E+21 ， 6.2062 E+21 ， 6.3063 E+21 ， 6.4064 E+21$,

$\& \quad 6.5065 \mathrm{E}+21 ， 6.6066 \mathrm{E}+21 ， 6.7067 \mathrm{E}+21 ， 6.8068 \mathrm{E}+21,6.9069 \mathrm{E}+21$ ，

$\& \quad 7.0070 \mathrm{E}+21,7.1071 \mathrm{E}+21,7.2072 \mathrm{E}+21,7.3073 \mathrm{E}+21,7.4074 \mathrm{E}+21$,

$\& \quad 7.5075 \mathrm{E}+21,7.6076 \mathrm{E}+21,7.7077 \mathrm{E}+21,7.8078 \mathrm{E}+21,7.9079 \mathrm{E}+21$,

$\& \quad 8.0081 \mathrm{E}+21,8.1082 \mathrm{E}+21,8.2083 \mathrm{E}+21,8.3084 \mathrm{E}+21,8.4085 \mathrm{E}+21$,

$\& \quad 8.5086 \mathrm{E}+21,8.6087 \mathrm{E}+21,8.7088 \mathrm{E}+21,8.8089 \mathrm{E}+21,8.9090 \mathrm{E}+21$,

$\& 9.0091 \mathrm{E}+21,9.1092 \mathrm{E}+21,9.2093 \mathrm{E}+21,9.3094 \mathrm{E}+21,9.4095 \mathrm{E}+21$,

$\& 9.5096 \mathrm{E}+21,9.6097 \mathrm{E}+21,9.7098 \mathrm{E}+21,9.8099 \mathrm{E}+21,9.9100 \mathrm{E}+21$,

$\& \quad 1.0010 \mathrm{E}+22,1.0110 \mathrm{E}+22,1.0210 \mathrm{E}+22,1.0310 \mathrm{E}+22,1.0410 \mathrm{E}+22$,

$\& \quad 1.0511 \mathrm{E}+22,1.0611 \mathrm{E}+22,1.0711 \mathrm{E}+22 ， 1.0811 \mathrm{E}+22 ， 1.0911 \mathrm{E}+22$ ，

$\& \quad 1.1011 \mathrm{E}+22,1.1111 \mathrm{E}+22,1.1211 \mathrm{E}+22,1.1311 \mathrm{E}+22,1.1411 \mathrm{E}+22$,

$\& \quad 1.1512 \mathrm{E}+22,1.1612 \mathrm{E}+22,1.1712 \mathrm{E}+22,1.1812 \mathrm{E}+22,1.1912 \mathrm{E}+22$,

$\& \quad 1.2012 \mathrm{E}+22,1.2112 \mathrm{E}+22,1.2212 \mathrm{E}+22,1.2312 \mathrm{E}+22,1.2412 \mathrm{E}+22$,

$\& \quad 1.2513 \mathrm{E}+22,1.2613 \mathrm{E}+22,1.2713 \mathrm{E}+22,1.2813 \mathrm{E}+22,1.2913 \mathrm{E}+22$, 
Table A.8. Code listing of RH7-ANSR (continued)

\begin{tabular}{|c|c|c|c|c|}
\hline$\&$ & 1. $3013 \mathrm{E}+22$, & 1. $3113 \mathrm{E}+22$, & $1.3213 \mathrm{E}+22,1.3313 \mathrm{E}+22$, & 1. $3413 E+22$, \\
\hline$\&$ & 1. $3514 \mathrm{E}+22$, & $1.3614 \mathrm{E}+22$, & $1.3714 \mathrm{E}+22,1.3814 \mathrm{E}+22$, & 1. $3914 \mathrm{E}+22$, \\
\hline$\&$ & 1. $4014 \mathrm{E}+22$, & 1. $4114 \mathrm{E}+22$, & 1. $4214 E+22$, & 1. $4414 \mathrm{E}+22$, \\
\hline$\&$ & 1. $4515 \mathrm{E}+22$, & 1. 4615E+22， & 1. $4715 \mathrm{E}+22$, & 1. $4915 \mathrm{E}+22$, \\
\hline$\&$ & 1. $5015 \mathrm{E}+22$, & 1. 5115E+22, & 1. $5215 \mathrm{E}+22$, & 1. $5415 \mathrm{E}+22$, \\
\hline$\&$ & $1.5516 \mathrm{E}+22$, & $1.5616 \mathrm{E}+22$, & $1.5716 \mathrm{E}+22$, & 1. $5916 \mathrm{E}+22$, \\
\hline \& & 1. $6016 \mathrm{E}+22$, & 1. $6116 \mathrm{E}+22$, & 1. $6216 \mathrm{E}+22$, & 1. $6417 \mathrm{E}+22$, \\
\hline$\&$ & 1. $6517 \mathrm{E}+22$, & $1.6617 \mathrm{E}+22$, & 1. $6717 \mathrm{E}+22$, & 1. $6917 \mathrm{E}+22$, \\
\hline$\&$ & $1.7017 \mathrm{E}+22$, & 1. 7117E+22, & 1. $7217 \mathrm{E}+22$ & 1. $7418 E+22$, \\
\hline$\&$ & 1. $7518 \mathrm{E}+22$, & 1. $7618 \mathrm{E}+22$, & $1.7718 \mathrm{E}+22$, & 1. $7918 \mathrm{E}+22$, \\
\hline$\&$ & 1. $8018 E+22$, & 1. $8118 E+22$, & 1. $8218 \mathrm{E}+22$, & 1. $8419 \mathrm{E}+22$, \\
\hline$\&$ & 1.8519E+22, & $1.8619 \mathrm{E}+22$, & $1.8719 \mathrm{E}+22$, & 1. $8919 \mathrm{E}+22$, \\
\hline$\&$ & 1.9019E+22, & 1.9119E+22, & 1.9219E+22, & 1. $9420 E+22$, \\
\hline$\&$ & 1.9520E+22, & 1.9620E+22, & 1.9820E+22, & $1.9920 \mathrm{E}+22$ \\
\hline$\&$ & $2.0020 E+22$ & $2.5 e+22 /$ & & \\
\hline & catab/7.913 & $31,7.9131,7$ & $8619,7.8190,7.7807$, & \\
\hline \& & 7.7456, & 7.6820, & 7.6245 & \\
\hline$\&$ & 7.5974, & 7.5460, & $7.5215,7.4976$ & \\
\hline$\&$ & 7.4743 , & 7.4294, & 7.4076 , & \\
\hline$\&$ & 7.3653, & 7.3137, & 7.2828 , & \\
\hline$\&$ & 7.2232 , & 7.1643 , & 7.1349, & \\
\hline$\&$ & 7.0761, & 7.0173, & $6.9879,6.9585$ & \\
\hline$\&$ & 6.9292, & 6.8706, & $6.8415,6.8124$, & \\
\hline$\&$ & 6.7834, & 6.7241, & 6.6936, & \\
\hline$\&$ & 6.6328, & 6.5721 , & $6.5419,6.5118$, & \\
\hline$\&$ & 6.4818, & 6.4222 , & $6.3926,6.3631$, & \\
\hline$\&$ & 6.3337 & 6.2755 & $6.2466,6.2179$, & \\
\hline 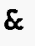 & 6.1894 & 6.1328, & $6.1048,6.0770$, & \\
\hline$\&$ & 6.0494 & 5.9947, & 5.9676 , & \\
\hline$\&$ & 5.9140, & 5.8611 , & $5.8349,5.8089$, & \\
\hline $\mathcal{\alpha}$ & 5.7831 , & 5.7331 , & $5.7084,5.6839$, & \\
\hline \& & 5.6596, & 5.6115, & $5.5877,5.5640$, & \\
\hline$\&$ & 5.5405 , & 5.4940, & $5.4709,5.4480$, & \\
\hline \& & 5.4252, & 5.3801 , & 5.3578 & \\
\hline$\&$ & 5.3135, & 5.2697, & $5.2480,5.2264$ & \\
\hline$\&$ & 5.2049 & 5.1623, & $5.1412,5.1202$, & \\
\hline$\&$ & 5.0993. & 5.0579, & $5.0373,5.0168$, & \\
\hline \& & 4.9964 & 4.9560 , & $4.9359,4.9159$, & \\
\hline$\&$ & 4.8960 , & 4.8565 , & $4.8369,4.8174$, & \\
\hline$\&$ & 4.7979. & 4.7593, & $4.7401,4.7210$ & \\
\hline$\varepsilon$ & 4.7019 , & 4. 6641, & $4.6453,4.6266$, & \\
\hline$\&$ & 4.6079, & 4.5708 , & $4.5524,4.5340$, & \\
\hline$\&$ & 4.5157, & 4.4793, & $4.4612,4.4432$, & \\
\hline \& & 4.4252 , & 4.3894 , & $4.3716,4.3539$, & \\
\hline$\&$ & 4.3363 , & 4.3011, & $4.2836,4.2662$, & \\
\hline$\&$ & 4.2488 & 4.2142 , & $4.1970,4.1799$, & \\
\hline$\&$ & 4.1628 & 4.1287, & $4.1118,4.0949$, & \\
\hline$\&$ & 4.0780 , & 4.0445 , & $4.0278,4.0111$, & \\
\hline$\&$ & 3.9945, & 3.9614 , & $3.9450,3.9285$, & \\
\hline$\varepsilon$ & 3.9122 , & 3.8795 , & $3.8633,3.8471$, & \\
\hline$\&$ & 3.8309, & 3.7987 , & $3.7827,3.7667$, & \\
\hline$\&$ & 3.7508 & 3.7190 , & $3.7031,3.6873$, & \\
\hline \& & 3.6716 , & 3.6402 , & $3.6245,3.6089$, & \\
\hline \& & 3.5934 & 3.5623 , & $3.5469,3.5314$, & \\
\hline$\&$ & 3.5160 & 3.4854 , & $3.4701,3.4548$, & \\
\hline$\&$ & 3.4396 & & & \\
\hline
\end{tabular}


Table A.8. Code listing of RH7-ANSR (continued)

c

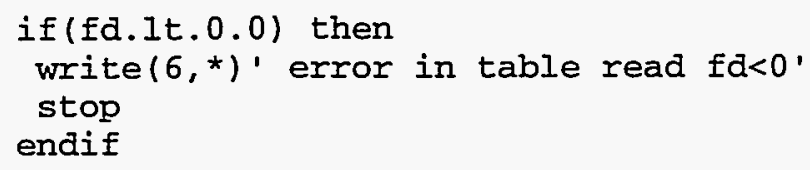

c

imatch $=0$

do $100 \quad i=1$, ifdlast

if(fd.ge.fdtab(i)) imatch $=i$

100 continue

if (fd.ge.fdtab(ifdlast)) then

fdratio $=$ fd/fdtab (ifdlast)

write $\left(6,{ }^{*}\right)$ ' $f d$ =maximum(seg) was', fd,fdratio

endif

$i=$ imatch

if(i.1e.0) then

c if $\mathrm{fd}=0$ then $\mathrm{cd}=\mathrm{cd} 0$

$\operatorname{cdi}=\operatorname{cdtab}(1)$

else if(i.ge.ifdlast) then

c if $\mathrm{fd}>\max$ then $\mathrm{cd}=\mathrm{cd}$ \& $\mathrm{fdmax}$

cdi $=$ cdtab (ifdlast)

else

$\operatorname{cdi}=\operatorname{cdtab}(i)+(\operatorname{cdtab}(i+1)-\operatorname{cdtab}(i))$

$\&$

*(fd-fdtab(i))/(fdtab(i+1)-fdtab(i))

endif

c convert to $\mathrm{W} / \mathrm{cmk}$

$\operatorname{cdi}=\operatorname{cdi} / 100$.

return

end

Cssssssssssssssssssssssssssssssssssssssssssssssssssssssssssssssssss subroutine sortg (a, lend)

c sort the array in acending and order remove duplicates real a $(40)$

data $\operatorname{maxl} / 40 /$

c sort a

do 300 io $=1$, lend

do $300 i=1$, lend -1

if $(a(i) \cdot g t \cdot a(i+1))$ then

adum $=a(i)$

$a(i)=a(i+1)$

$a(i+1)=$ adum

endif

300 continue

c

omit duplicates from sorted list

lendn = lend

do 400 io $=1$, lendn-1

lendni $=$ lend

do $360 i=1$, lendni-1

if $(i+1 . g t . l e n d)$ go to 400

if $(a b s(a(i)-a(i+1)) \cdot 1 e \cdot 1.0 d-5)$ then

do $350 \quad i i=i+1$, lend -1

$350 \quad a(i i)=a(i i+1)$

$a($ lend $)=0$

lend $=$ lend -1

endif

continue 
A. 41

Table A.8 Code listing of RH7-ANSR (continued)

400 continue return end 
A.42

\section{Table A.9. Code listing of DATIM.C}

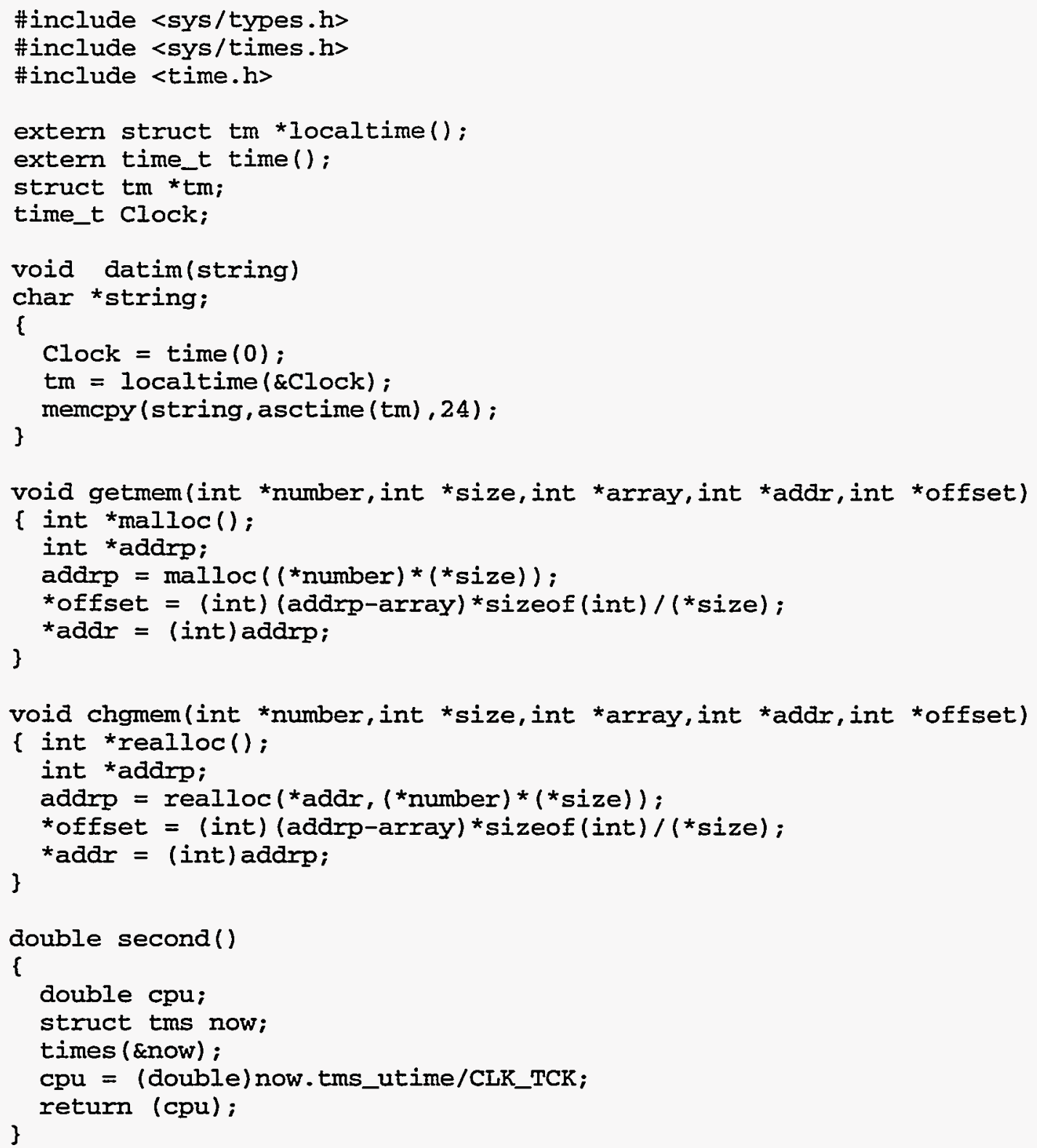




\section{APPENDIX B \\ CRITICAL HEAT FLUX ANALYSIS}

The critical heat flux condition is characterized by a large reduction in local heat transfer coefficient that results from the displacement of liquid by vapor next to the surface. The existence of a vapor bubble on the surface at the point of maximum heat flux would produce less effective heat transfer over a portion of the surface above the defect pair. A reduction in heat transfer effectiveness will tend to increase the local heat flux. This investigation was conducted to determine if the vapor region would likely increase in size or be limited by natural conditions.

A model for a fuel defect pair in a maximum thickness meat layer was modified by placing an adiabatic region on the hot-side surface of the clad, Fig. B.1. This region simulates the formation of a steam bubble on the hot side of the fuel plate due to a hypothetical system change. The vapor region will reduce the heat transfer from the surface to the liquid. An adiabatic condition was a conservative assumption for the prediction of bubble size reduction. This analysis should overpredict the heat flux at the edge of the bubble because of this conservatively low assumption of the heat transfer. Thus if the analysis were to predict that the heat flux at the edge of a bubble was not significantly higher than a slightly smaller bubble, the bubble should not grow in size. In actuality the bubble should reduce in size and produce a natural limiting condition. Such a condition would indicate that vapor bubbles would not increase in size without other external conditions changing. This stability of the bubble could be an important benefit, allowing higher operating conditions. However, the conclusions from the investigation were not definitive.

Cylinder segregation, $0.762 \mathrm{~mm}$ meat

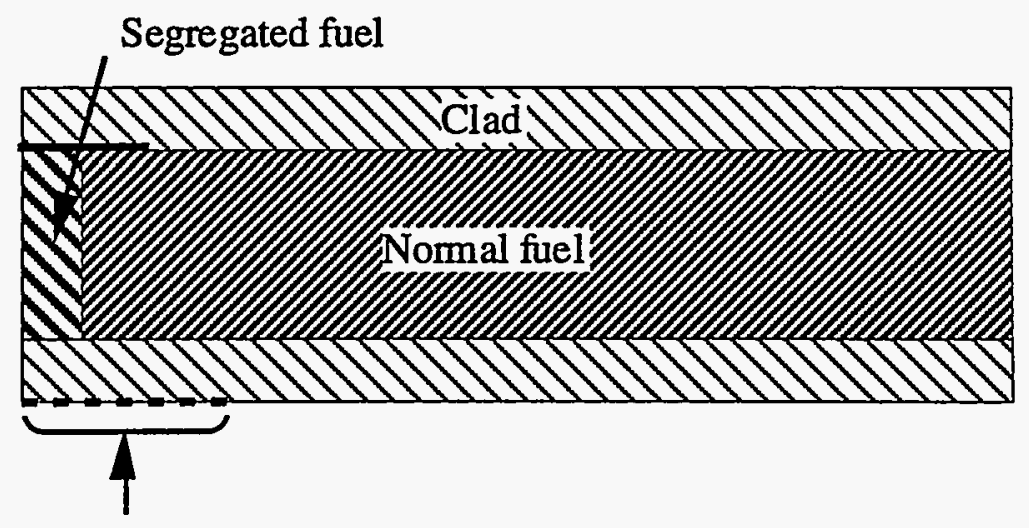

Adiabatic steam bubble

Fig. B.1. Critical heat flux analysis model. 
The peaking factor for this conservative analysis increased sharply as the bubble size was increased, Fig. B.2. This rapid rise in the local heat flux at the edge of the adiabatic bubble indicates that once formed the bubble will increase in size as long as the peaking factor is above the initial value. The largest radius analyzed was $4.0 \mathrm{~mm}$ and the peaking factors were continuing to increase. For much larger bubbles, it may be possible for the peaking factor to be lower than the initial value. However, this may not be a very useful result because of the flow disruption by the bubble. It is likely that the bubble will have a height that is a large fraction of the diameter. A bubble 2 to $4 \mathrm{~mm}$ high will adversely disrupt the flow in the $12.7-\mathrm{mm}$-high channels and invalidate the assumption that the heat transfer coefficients not under the bubble are the same as for undisturbed flow. Reducing these coefficients would increase the tendency for the bubble to increase in size. Thus a potential limiting condition that may exist above $4 \mathrm{~mm}$ diam in the analysis would not be realistic.

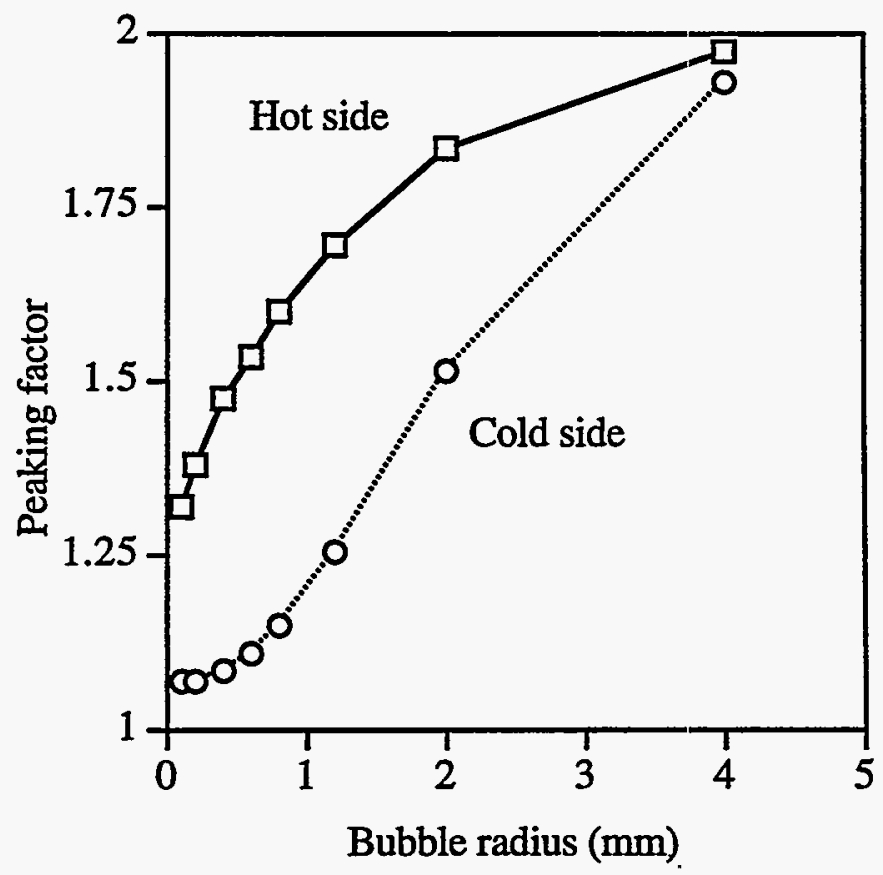

Fig. B.2. Maximum peaking factor as a function of the size of an adiabatic region on the surface on the model.

The rapid rise in the cold side peaking factor indicates that an additional bubble will form on the opposite side above a radius of $1.5 \mathrm{~mm}$. This will further accelerate the bubble growth on the hot-side by restricting the plate cooling from the cold-side. 
It is possible that reducing the conservatism of the model by the inclusion of a more accurate heat transfer across the vapor region could reduce the rapidity of this increase and alter the conclusions. Large uncertainties in these heat transfer mechanisms under the critical heat flux condition make it difficult to develop an analysis that would be more conclusive.

The reduction in heat transfer effectiveness on the hot side of the model also adversely affects the maximum temperatures in this analysis. Figure B. 3 presents these temperatures. These analyses were performed with an adaptation of the L7 end-of-cycle models. The segregated material was assumed to be $50 \% \mathrm{U}_{3} \mathrm{Si}_{2}$.

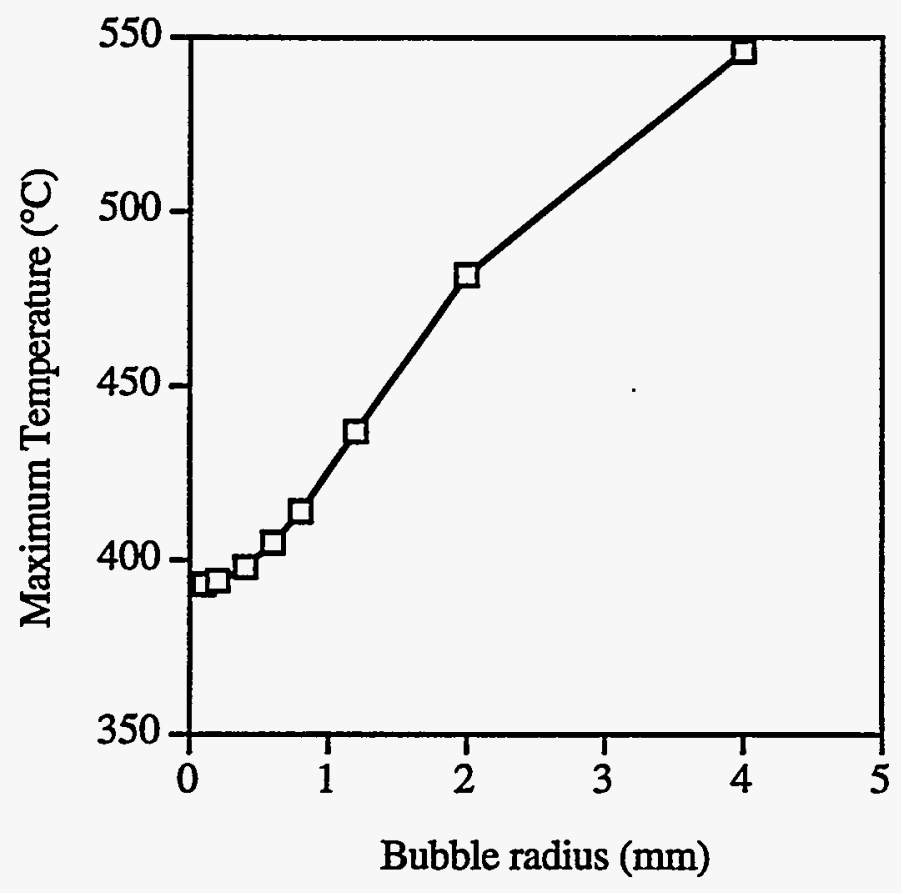

Fig. B.3. Maximum temperature in the center of segregation volume as a function of the size of an adiabatic region on the surface on the model. 


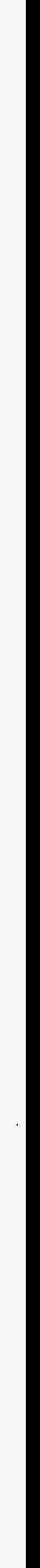




\section{APPENDIX C FUNCTIONAL REPLACEMENT FOR HEATING ANALYSES}

The TASHA code is used for ANSR Safety Analyses. It determines the operational limits that should be imposed on the ANSR to maintain safe conditions. The thermal models in TASHA are a collection of 1-D idealized models. TASHA uses the modified heat flux to calculate the local maximum fuel temperature. In the TASHA mode, the local heat flux is modified from nominal by the hot spot and heat generation uncertainty factors. The thermal conductivity of the clad, filler and meat is then used in a one-dimensional model to calculate the maximum fuel temperature. This temperature will be greater than that expected for normal fuel (without a defect pair) at the same location because of the use of the hot spot uncertainty. However, because the lower conductivity of the segregated fuel is not considered, this model underestimates the maximum temperatures. In addition, the peaking factor used in TASHA is fixed for all points and all time steps. This assumption is overly conservative, as shown in Sect. 4. It may be necessary to improve the TASHA code to more accurately predict local fuel temperatures.

The next logical improvement of the TASHA code would be to include a more accurate thermal model to determine the local peaking factor and maximum temperatures. It was felt that directly including the HEATING type of fuel defect analysis might require excessive computational resources.

As an alternative approach, a reasonably accurate correlation of peaking factor as a function of the thermal modeling parameters might be possible. This possibility was investigated by using the results from a selection of cases that encompass the maximum range of five variables and attempting to fit several functions to these data. The variables investigated included relative power density, meat thickness, fission density, coolant temperature and oxide thickness. The ranges used to produce the correlations are shown in Table C.1. A set of 3125 cases was generated by varying each parameter over five values spanning the minimum to maximum. Each parameter value is used in combination with every parameter value in the set. This data set is called the permutation data set.

Although several functional forms were investigated, a fairly simple function was found to achieve a reasonable fit with these data. The correlation for peaking factor is described in Eq.(C.1). The coefficients and exponents in this fit were determined from a multivariable optimization technique. The technique used was a straightforward minimum search method. The minimum value of the first of the optimization variables was determined. This new value was used in the minimization of the second. 
This process was repeated for each variable to complete a calculation cycle. After the second calculation cycle the residuals are checked to determine convergence. As long as the residuals are decreasing the cycles are continued. This technique can be very sensitive to the starting conditions. Various starting conditions were used to have more assurance that the local minimum that is found is truly the global minimum. The correctness of the fit was tested by performing a fit using the SAS system nonlinear optimization (NLIN) procedure. The results were nearly identical. Although the SAS NLIN procedure has a more rigorous procedure for finding the global minimum, both techniques agree on the minimum values.

Table C.1 Ranges of parameters

\begin{tabular}{lll}
\hline Parameter & Minimum & Maximum \\
\hline Power density & 0.3 & 2.3534 \\
Fission density & 0 & $2.4651 \times 10^{22}$ \\
Meat thickness & $0.1778 \mathrm{~mm}$ & $0.762 \mathrm{~mm}$ \\
Coolant temperature & $45^{\circ} \mathrm{C}$ & $130^{\circ} \mathrm{C}$ \\
Oxide thickness & $0 \mu \mathrm{m}$ & $30 \mu \mathrm{m}$ \\
\hline
\end{tabular}

Although several functional forms were investigated, a fairly simple function was found to achieve a reasonable fit with these data. The correlation for peaking factor is described in equation C.1. The coefficients and exponents in this fit were determined from a multi-variable optimization technique. The technique used was a straightforward minimum search method. The minimum value of the first of the optimization variables was determined. This new value was used in the minimization of the second. This process was repeated for each variable to complete a cycle. After the second cycle the residuals are checked to determine convergence. As long as the residuals are decreasing the cycles are continued. This technique can be very sensitive to the starting conditions. Various starting conditions were used to have more assurance that the local minimum that is found is truly the global minimum. The correctness of the fit was tested by performing a fit using the NLIN procedure. The results were nearly identical. Although the NLIN procedure has a more rigorous procedure for finding the global minimum, both techniques agree on the minimum values.

The correlation produced peaking factors are plotted against the HEATING produced peaking factors in Fig. C.1. The pattern shows a reasonable fit. The coolant temperature is not used in this peaking factor correlation because testing showed this parameter was not needed. 


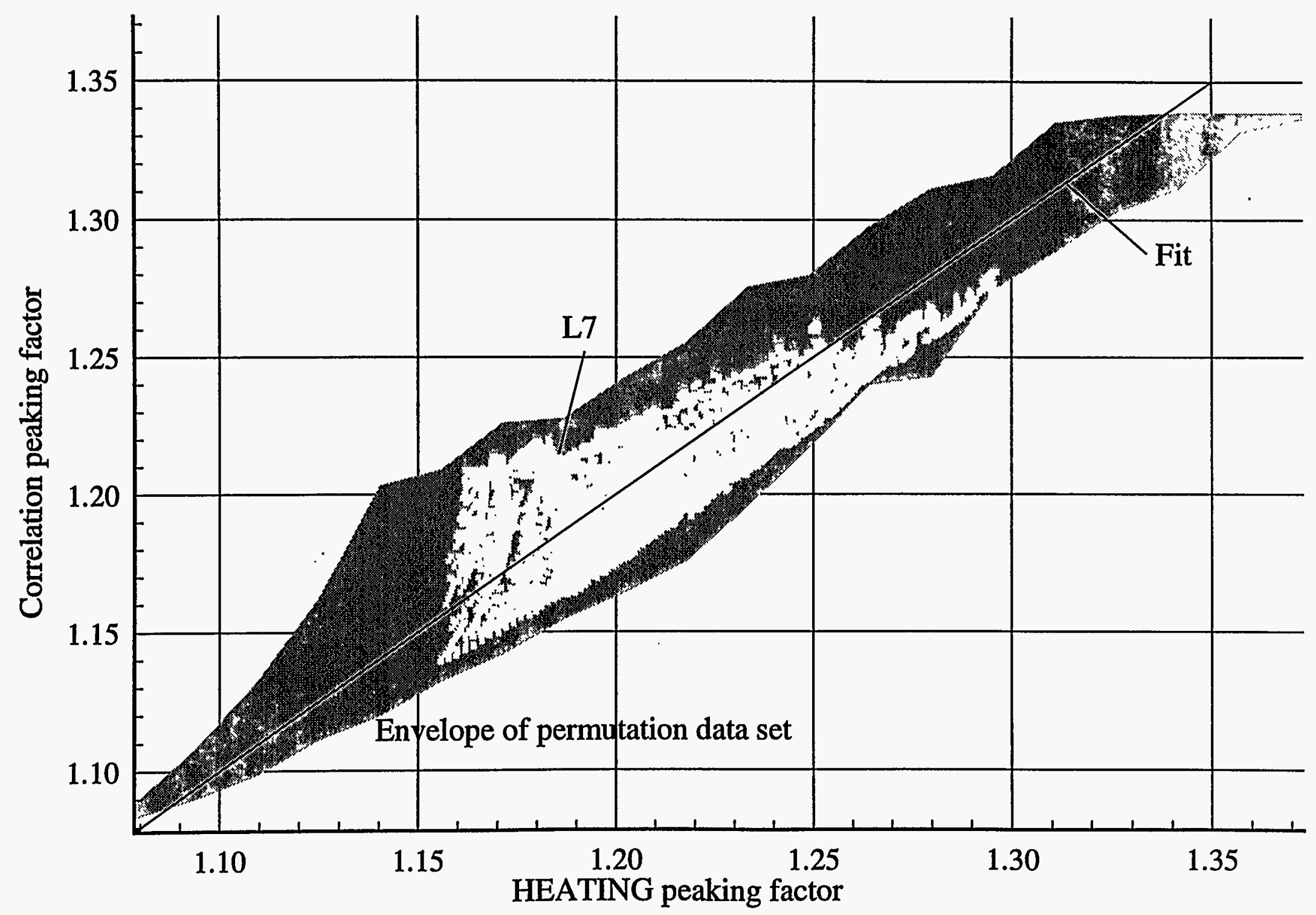

Fig. C.1 Peaking factor correlation from permutation data set compared with L7-best data 


$$
\begin{gathered}
\mathrm{C} .4 \\
\mathrm{Cp} \mathrm{p}_{\mathrm{f}}=\mathrm{c}_{1}+\mathrm{c}_{2}\left(\mathrm{p}_{\mathrm{d}}^{\mathrm{e}_{1}} \mathrm{k}_{\mathrm{f}}^{\mathrm{e}_{2}} \mathrm{k}_{\mathrm{s}}^{\mathrm{e}_{3}} \mathrm{t}_{\mathrm{m}}^{\mathrm{e}_{4}} \mathrm{x}_{\mathrm{o}}^{\mathrm{e}_{5}}\right),
\end{gathered}
$$

where

$\mathrm{Cp}_{\mathrm{f}}=$ Correlation produced peaking factor

$\mathrm{p}_{\mathrm{d}}=$ Relative power density

$\mathrm{k}_{\mathrm{f}}=$ Thermal conductivity of normal fuel $\left(\mathrm{W} / \mathrm{mm}^{\circ} \mathrm{C}\right)$ calculated from fission density

$k_{s}=$ Thermal conductivity of segregated fuel $\left(\mathrm{W} / \mathrm{mm}^{\circ} \mathrm{C}\right)$ calculated from fission density

$\mathrm{t}_{\mathrm{m}}=$ Meat thickness (mm)

$\mathrm{x}_{\mathrm{o}}=$ Oxide layer thickness $(\mu \mathrm{m})$

$\mathrm{c}_{1}=0.5081$

$c_{2}=0.3969$

$\mathrm{e}_{1}=0.0043$

$\mathrm{e}_{2}=-0.0683$

$\mathrm{e}_{3}=0.0136$

$\mathrm{e}_{4}=-0.0537$

$\mathrm{e}_{5}=-0.0512$

This gives

$$
\mathrm{Cp}_{\mathrm{f}}=0.5081+0.3969\left(\mathrm{p}_{\mathrm{d}}^{0.0043} \mathrm{k}_{\mathrm{f}}^{-0.0683} \mathrm{k}_{\mathrm{s}}^{0.0136} \mathrm{t}_{\mathrm{m}}^{-0.0537} \mathrm{x}_{\mathrm{o}}^{-0.0512}\right)
$$

The correlation in Fig. C.1 appears to be very reasonable but was generated for the permutation data set. As described above this data set was distributed evenly on the ranges of each variable. These data may not be representative of a specific core design and, due to the nonlinear nature of the combinatory effects of the parameters, may not encompass the worst possible points. To check for these conditions, the data from the TASHA code for the L7 core design using nominal assumptions were used to generate local maximum temperatures. These data were plotted in this same manner in Fig. C.2. The L7 data (solid symbol) is encompassed by the maximum range data, and the correlation appears to be reasonable for both sets of data. A slightly different correlation using the L7 data would have a better quality of fit to these data. However, the most appropriate use of this correlation would be as a conservative estimate function and only a small but perhaps significant improvement would be gained by using this more limited correlation.

This correlation can be used to calculate a conservative peaking factor that is a function of the five parameters by adding the maximum positive residual as a factor to make certain that the correlation will always produce peaking factors greater than or equal to those produced by HEATING. 
The various residuals are shown in Table C. 2 for several data sets. The permutation data set is described above and should encompass all the other data. The L7-nominal data set was generated from TASHA results for the L7 core design using the nomial values in the LAT. The L7-95\% data set was generated by using the TASHA results from all time steps assuming the $95 \%$ probability limits on the uncertainty factors in the TASHA code. These conditions were used in HEATING to generate maximum temperatures, assuming that the coolant temperature was a uniform $90^{\circ} \mathrm{C}$. This data set also includes results using the TASHA conditions but calculating the oxide thickness from the HEATING maximum heat flux (L7-calox). All data sets are encompassed by the permutations data set.

Table C.2. Fit quality of peaking factor correlation for various data sets

\begin{tabular}{lcccc}
\hline Residuals & Permutation & L7-best & L7-calox & L7-95\% \\
\hline Max pos & 0.03471 & 0.02725 & 0.02830 & 0.0350 \\
Max neg & -0.05729 & -0.04721 & -0.04371 & -0.0460 \\
RMS & 0.01527 & 0.02126 & 0.02264 & 0.0190 \\
AVE & -0.00001255 & -0.00333 & -0.005843 & 0.007340 \\
\hline
\end{tabular}

Residual = HEATING peaking factor - correlation peaking factor

RMS = Square root of mean of sum of the squares of residuals

\section{Correlation for maximum temperature}

This same multivariable nonlinear optimization technique was used to find a correlation for the maximum temperature as a function of the five parameters. Again, several trial functions were examined, and the best is reported in Eq.(C.2). The components of this function are the temperature difference for the 1-D flow of heat across the oxide layer, clad and filler layers and across the segregation spot and normal fuel thicknesses. The coefficients found by the optimization represent a linear combination of these terms and the comparison of the correlation and HEATING produced temperatures is shown in Fig. C.3. The L7-best data set is again plotted in the same manner to ensure the appropriateness of the correlation in Fig. C.4. 


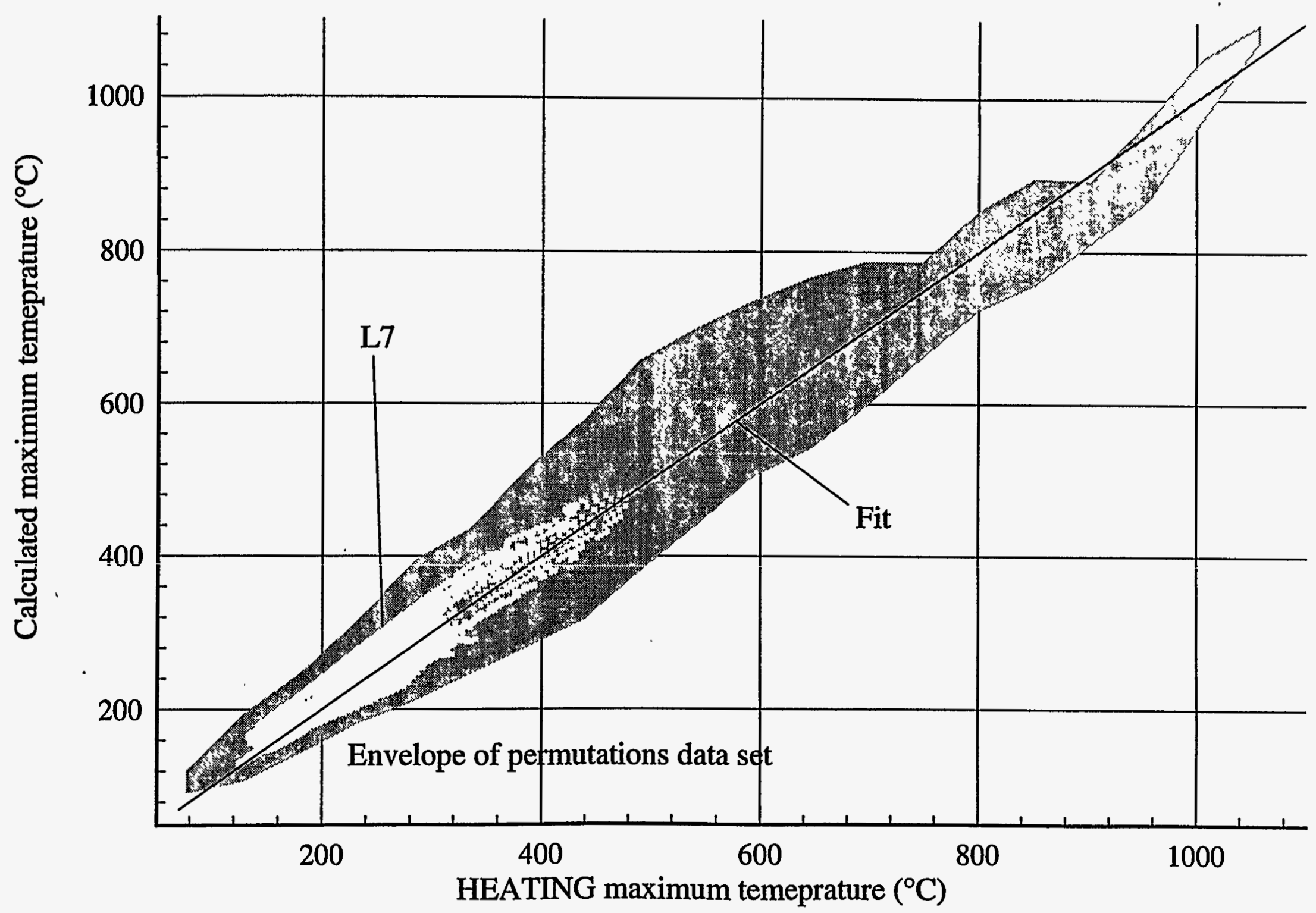

a

Fig. C.2 Maximum temperature correlation of permutation data set compared with L7-best data 


$$
\begin{aligned}
C t_{\max }= & t_{b}+d+a\left(q_{s e g} \frac{t_{m}}{k_{s}}\right)+b \frac{q_{s e g}}{2}\left(\frac{0.0254+\left(\frac{t_{m 0}-t_{m}}{2}\right)}{k_{c}}\right)+ \\
& c\left(\frac{q_{s e g}}{2}\right)\left(\frac{x_{0}}{k_{0}}\right)+e\left(q_{\frac{k_{m}}{k_{f}}}\right),
\end{aligned}
$$

where

$$
\begin{aligned}
& \mathrm{Ct}_{\max }=\text { Correlation produced maximum temperature }{ }^{\circ} \mathrm{C} \\
& t_{b}=\text { Coolant temperature }{ }^{\circ} \mathrm{C} \\
& \mathrm{q}_{\text {seg }}=\text { Local heat generation rate of segregated fuel } \mathrm{W} / \mathrm{cm}^{3} \\
& \mathrm{k}_{\mathrm{o}}=\text { Thermal conductivity of the oxide }=0.225 \mathrm{~W} / \mathrm{cm}^{\circ} \mathrm{C} \\
& \mathrm{tm}_{0}=\text { Maximum meat thickness } 0.762 \mathrm{~mm} \\
& \mathrm{k}_{\mathrm{c}}=\text { Thermal conductivity of the clad and filler }=1.7 \mathrm{~W} / \mathrm{cm}^{\circ} \mathrm{C} \\
& \mathrm{q}=\text { Local heat generation of normal fuel W/cm }{ }^{3} \\
& \mathrm{a}=0.00359 \\
& \text { b }=0.015459 \\
& \text { c }=0.064247 \\
& \mathrm{~d}=22.48 \\
& \mathrm{e}=0.030816
\end{aligned}
$$

This gives:

$$
\begin{aligned}
\mathrm{C} \mathrm{t}_{\max }= & \mathrm{t}_{\mathrm{b}}+22.48+0.00359\left(\mathrm{q}_{\mathrm{seg}} \frac{\mathrm{t}_{\mathrm{m}}}{\mathrm{k}_{\mathrm{s}}}\right)+0.015459 \frac{\mathrm{q}_{\mathrm{seg}}}{2}\left(\frac{0.0254+\left(\frac{\mathrm{t}_{\mathrm{m} 0}-\mathrm{t}_{\mathrm{m}}}{2}\right)}{\mathrm{k}_{\mathrm{c}}}\right) \\
& + \\
& 0.064247\left(\frac{\mathrm{q}_{\mathrm{seg}}}{2}\right)\left(\frac{\mathrm{x}_{\mathrm{o}}}{\mathrm{k}_{\mathrm{o}}}\right)+0.030816\left(\mathrm{q}_{\mathrm{k}_{\mathrm{f}}}\right)
\end{aligned}
$$

This correlation can be used to calculate a conservative maximum temperature that is a function of the five parameters by adding the maximum positive residual as a factor to ensure that the correlation will always produce maximum temperatures greater than or equal to those produced by HEATING. The various residuals are shown in Table C.3 for several data sets. All data sets are encompassed by the permutations data set.

The heat transfer model in this investigation did not include the possibility of the conversion of the segregated fuel from $\mathrm{U}_{3} \mathrm{Si}_{2}$ to $\mathrm{U}(\mathrm{Al}, \mathrm{Si})_{3}$. The volume of the converted material and the conductivity would have to be added to the correlation to be consistent with the current theories on the thermal behavior of the fuel. An estimate of the maximum extent of the 


\section{C.8}

$\mathrm{U}(\mathrm{Al}, \mathrm{Si})_{3}$ region would have to be modeled in the TASHA code in order to make use of this new function. This estimate would be difficult to produce, and the potential inaccuracies might reduce the usefulness of a functional representation.

Table C.3.

Fit quality of maximum temperature correlation for various data sets

\begin{tabular}{lcccc}
\hline Residuals & Permutation & L7-best & L7-calox & L7-95\% \\
\hline Max pos & 108.49 & 49.33 & 44.97 & 99.07 \\
Max neg & -151.7 & -52.25 & -62.09 & -99.39 \\
RMS & 35.07 & 19.8 & 20.49 & 26.49 \\
AVE & -0.018 & -7.74 & -8.11 & -8.21 \\
\hline \multicolumn{1}{c}{ Residual }
\end{tabular}

Residual = HEATING max. temperature - correlation max. temperature 


\section{APPENDIX D \\ ADVANCED NEUTRON SOURCE REACTOR CORE DESIGN DATA}

This appendix contains a series of figures that describe the data comprising the L7 and G693 core designs. These data include the distribution on the surface of the two plates of the local meat thickness, relative power density, fission density, oxide layer thickness and coolant temperature.

\section{D.1 L7 CORE DESIGN FUEL PLATES}

A summary of the data used in the LAT analyses of the L7 core design is presented in the section.

The meat thickness remains constant over the cycle, and the distribution is shown in Fig. D.1. Distributions for both the upper and lower fuel plates are shown in this figure. The thickness colors are the same for both plates.

Figures D.2 to D.8 present the time-dependent data for the power density, thermal conductivities, oxide thickness and coolant temperatures. In these figures, the upper- and lower-plate pair is shown for every time step. The first time step pair is at the bottom of the figure. The plate-pair for the next time step is just above. This sequence is repeated with the last time step plate-pair at the top of the figure. The same color spectrum is used for all plates for comparison. These figures show the magnitude of the nonuniformity in both space and time that exist in the ANSR fuel plates. The oxide thickness distributions for both the 95\% limits and the "best estimate" TASHA analyses are also shown. The coolant temperatures are presented for only the "best estimate" case.

Tables D.1 to D.4 present a summary of the time dependent data of the $\mathrm{L} 7$ core design.

Table D.1. L7 Relative Power and Fission Density

\begin{tabular}{lllll}
\hline $\begin{array}{l}\text { Time } \\
\text { (d) }\end{array}$ & \multicolumn{2}{l}{$\begin{array}{l}\text { Relative power density } \\
\text { Min }\end{array}$} & Max & \multicolumn{2}{l}{$\begin{array}{l}\text { Fission density }\left(\mathrm{f} / \mathrm{mm}^{3}\right) \\
\text { Min }\end{array}$} \\
\hline 0 & 0.34384 & 2.3534 & 0.0 & 0.0 \\
4.25 & 0.42518 & 2.3274 & $0.12836 \mathrm{E}+22$ & $0.89479 \mathrm{E}+22$ \\
8.5 & 0.50867 & 2.1191 & $0.26271 \mathrm{E}+22$ & $0.16221 \mathrm{E}+23$ \\
12.75 & 0.56350 & 1.9323 & $0.40919 \mathrm{E}+22$ & $0.21962 \mathrm{E}+23$ \\
17 & 0.35813 & 1.8130 & $0.58703 \mathrm{E}+22$ & $0.26421 \mathrm{E}+23$ \\
\hline
\end{tabular}


Table D.2. L7 Fuel Conductivities

\begin{tabular}{lllll}
\hline \multirow{2}{*}{$\begin{array}{l}\text { Time } \\
\text { (d) }\end{array}$} & \multicolumn{3}{c}{ Thermal Conductivity $\left(\mathrm{W} / \mathrm{cm}^{\circ} \mathrm{C}\right)$} \\
& Normal fuel & \multicolumn{3}{c}{ Segregated fuel } \\
& Min & Max & Min & Max \\
\hline 0 & 1.7 & 1.7 & 0.145 & 0.145 \\
4.25 & 1.193 & 1.676 & 0.127 & 0.546 \\
8.5 & 0.838 & 1.564 & 0.162 & 0.547 \\
12.75 & 0.548 & 1.456 & 0.17 & 0.547 \\
17 & 0.394 & 1.346 & 0.1 & 0.547 \\
\hline
\end{tabular}

Table D.3.

L7 TASHA 95\% Limits Case Oxide Thickness and Coolant Temperature

\begin{tabular}{lll}
\hline Time & \multicolumn{2}{l}{ Oxide layer thickness $(\mu \mathrm{m})$} \\
$(\mathrm{d})$ & Min & Max \\
0 & 0.0 & 0.0 \\
4.25 & 0.293 & 17.073 \\
8.5 & 0.573 & 21.158 \\
12.75 & 0.876 & 22.14 \\
17 & 1.431 & 22.334 \\
\hline
\end{tabular}

Table D.4.

L7 TASHA "best estimates" Case Oxide Thickness and Coolant Temperature

\begin{tabular}{lllll}
\hline $\begin{array}{l}\text { Time } \\
\text { (d) }\end{array}$ & \multicolumn{2}{l}{ Oxide layer thickness $(\mu \mathrm{m})$} & \multicolumn{2}{l}{ Coolant temperature $\left({ }^{\circ} \mathrm{C}\right)$} \\
& Min & Max & Min & $\operatorname{Max}$ \\
\hline 0 & 0.0 & 0.0 & 45 & 114.64 \\
4.25 & 0.272 & 7.0823 & 45 & 104.36 \\
8.5 & 0.540 & 8.8886 & 45 & 93.34 \\
12.75 & 0.766 & 9.4915 & 45 & 106.65 \\
17 & 0.910 & 9.4915 & 45 & 106.65 \\
\hline
\end{tabular}




\section{D.3}

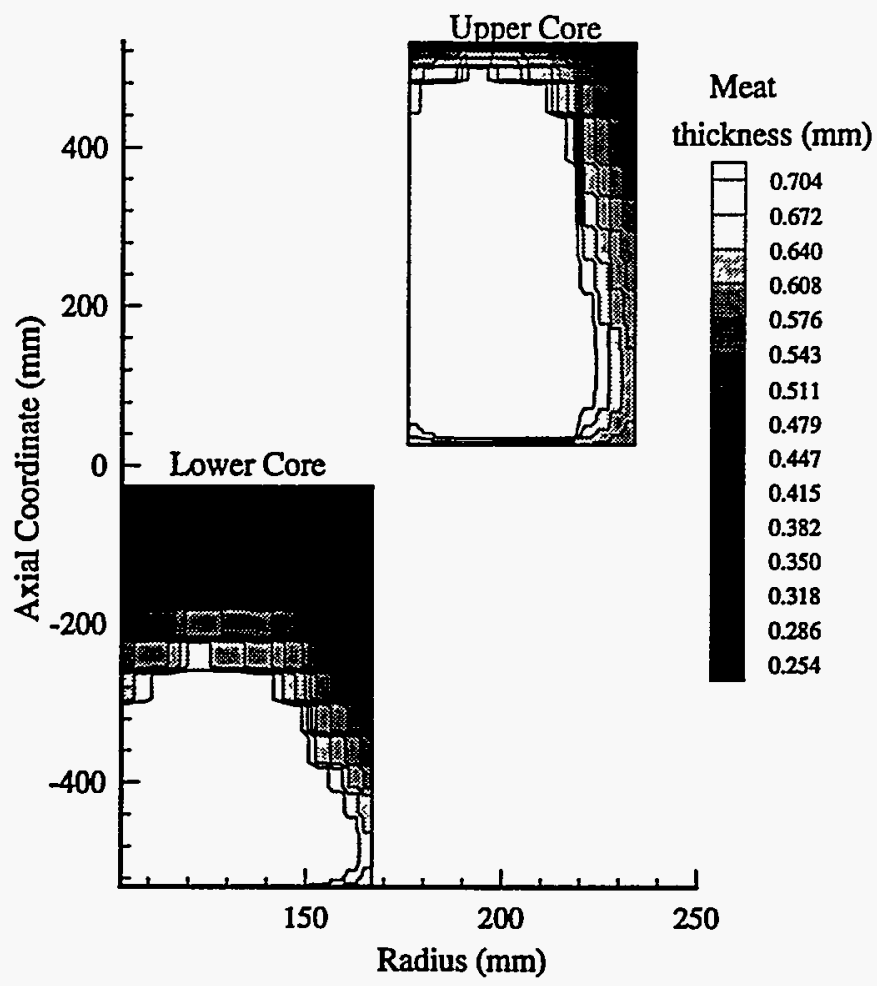

Fig. D.1 Meat thickness distribution for the L7 core design. 


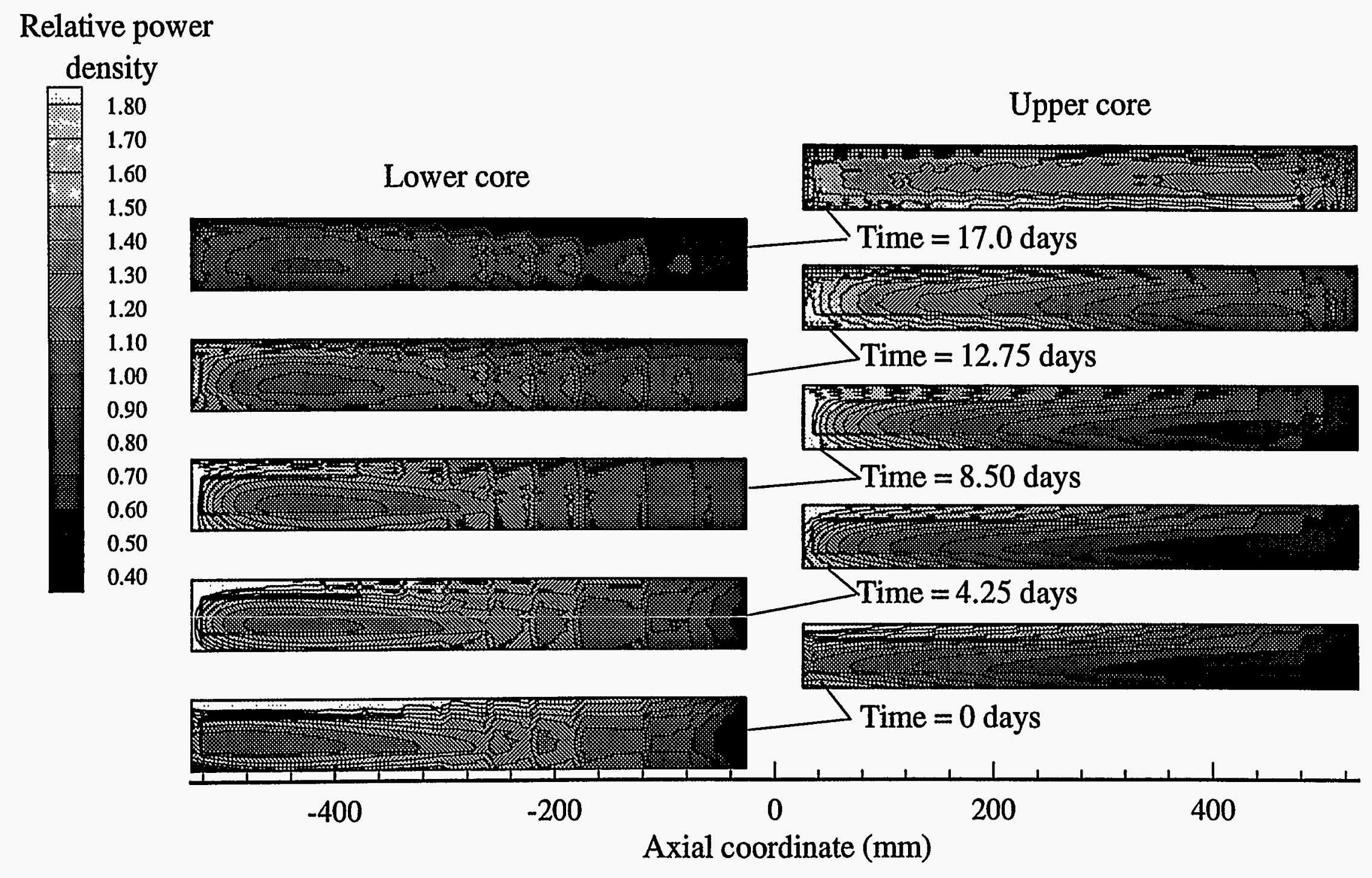

Fig. D.2. Relative power density distribution on L7 plates, all time steps. 


\section{Conductivity}

$\left(\mathrm{W} / \mathrm{cm}^{\circ} \mathrm{C}\right)$
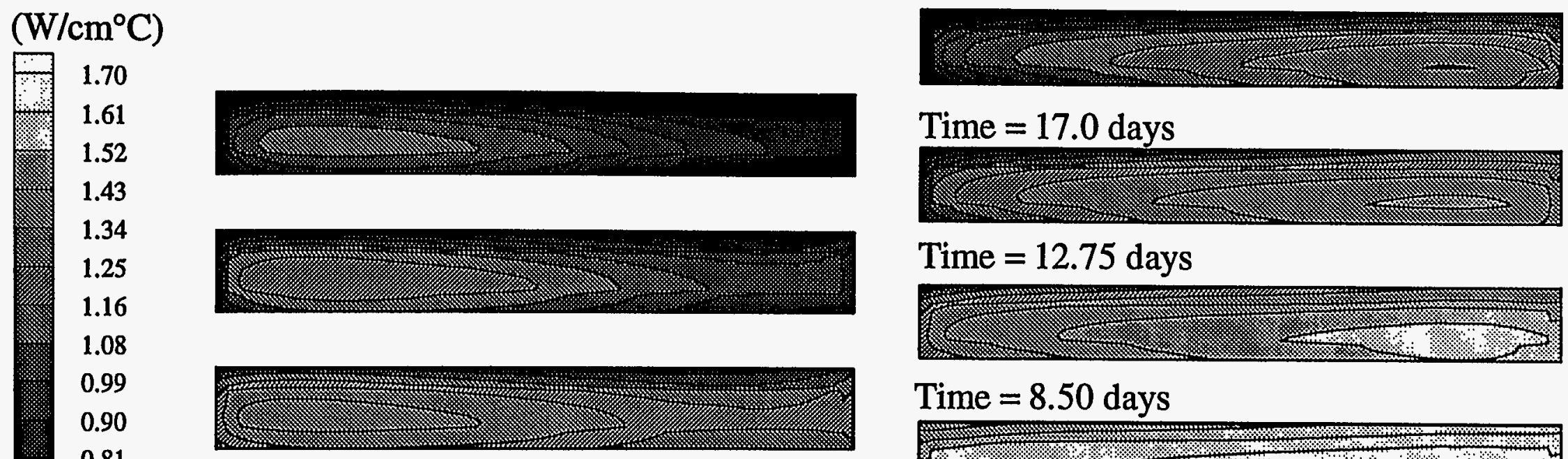

Time $=17.0$ days

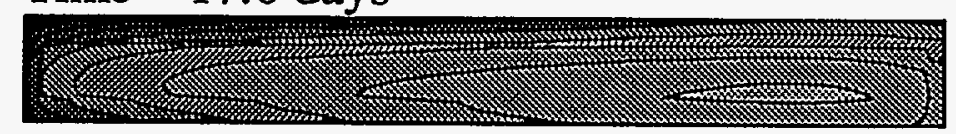

Time $=12.75$ days

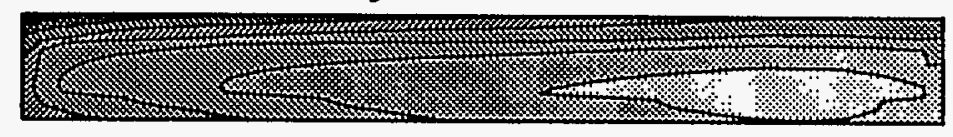

Time $=8.50$ days

0.81

0.72

0.63

0.54

0.45
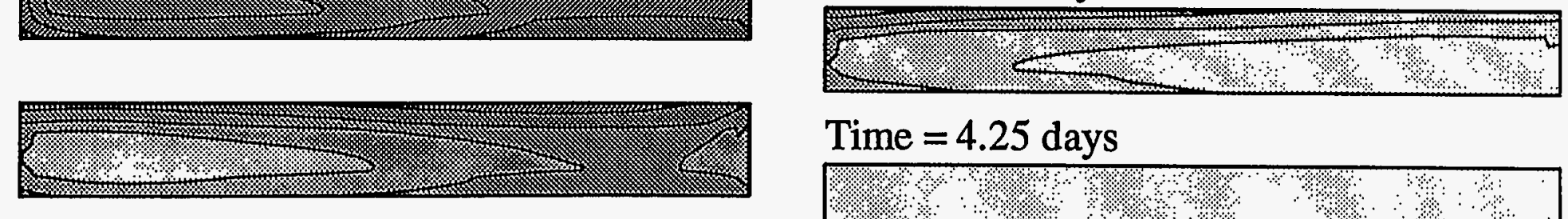

Time $=4.25$ days
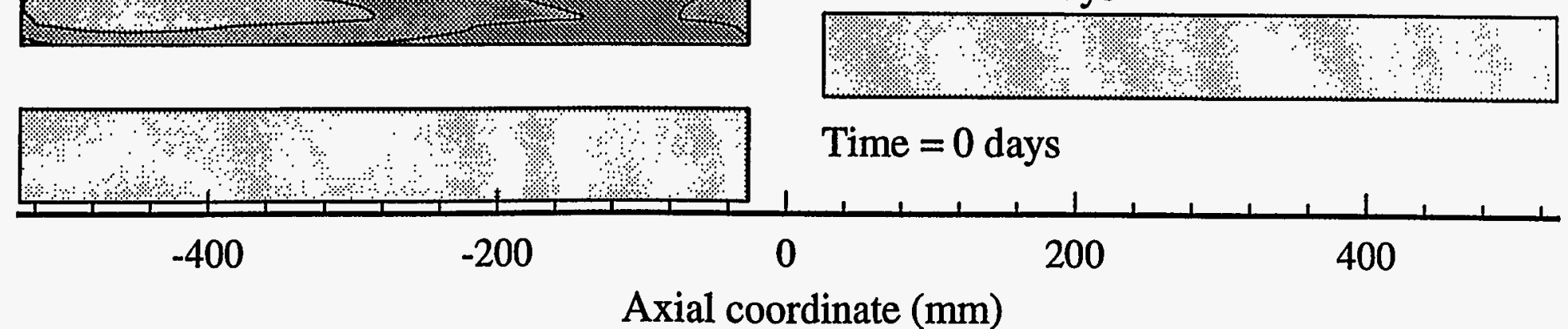

Fig. D.3. Contours of themal conductivity of normal fuel for each time step, L7 core design. 
Conductivity

$\left(\mathrm{W} / \mathrm{cm}^{\circ} \mathrm{C}\right)$

0.55
0.52
0.49
0.45
0.42
0.39
0.36
0.33
0.29
0.26
0.23
0.20
0.16
0.13
0.10
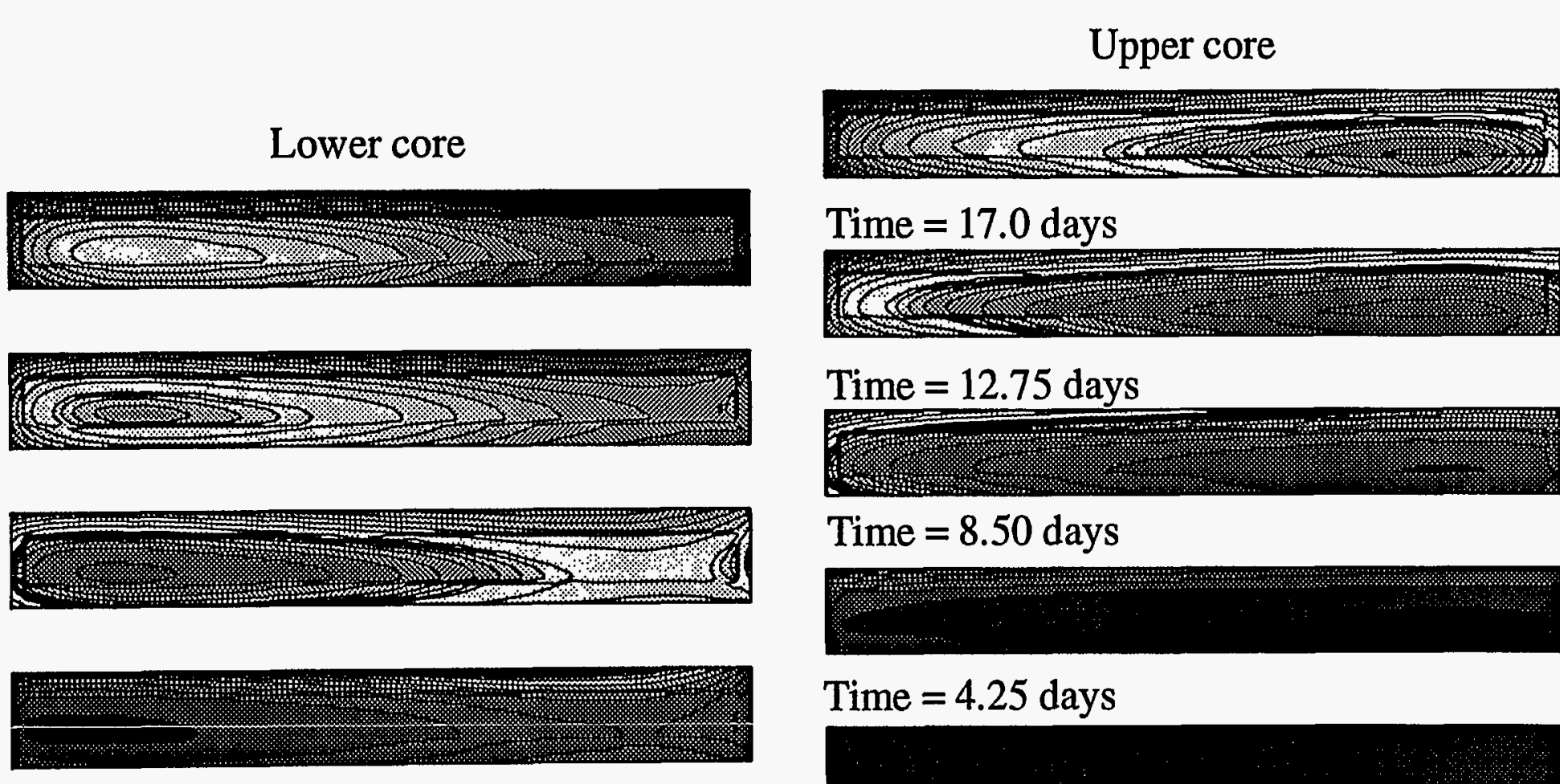

Time $=4.25$ days

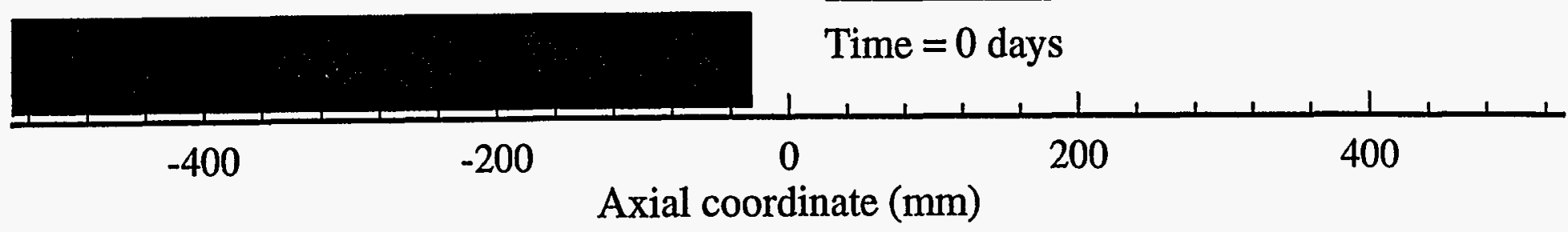

Fig. D.4. Contours of thermal conductivity of segregated fuel, L7 design. 
Fission density

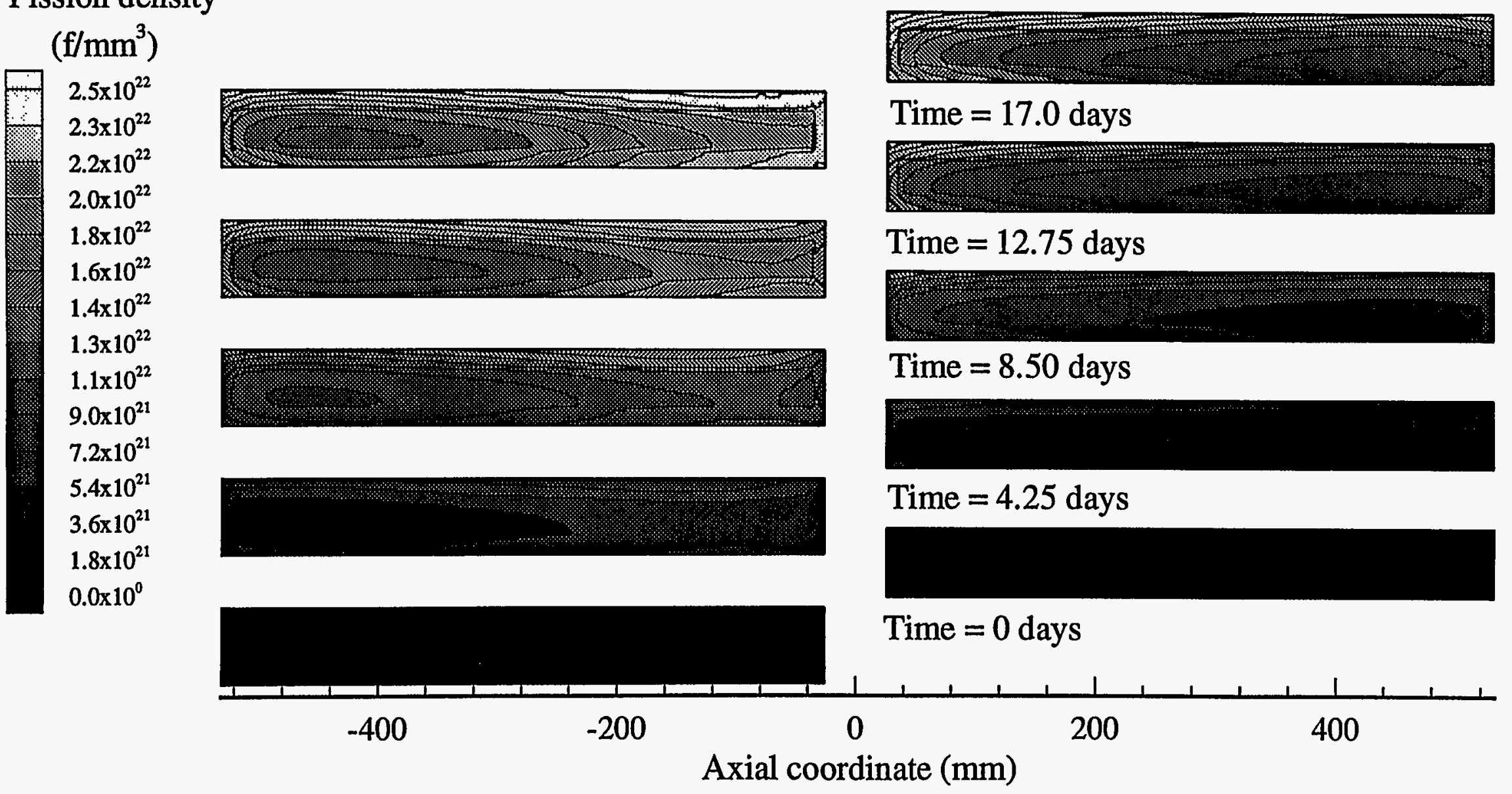

Fig. D.5. Countours of fission density, L7 design, 95\% limits TASHA case. 
Thickness $(\mu \mathrm{m})$

21
19.5
18
16.5
15
13.5
12
10.5
9
7.5
6
4.5
3
1.5
0

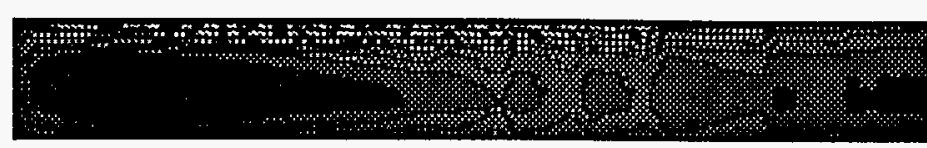

Time $=17.0$ days

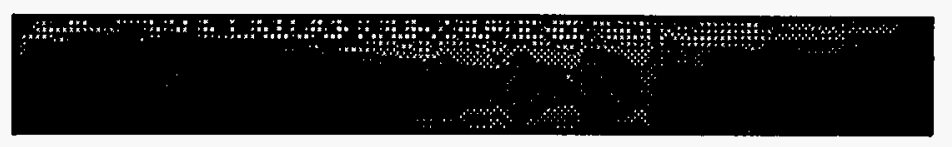

Time $=12.75$ days

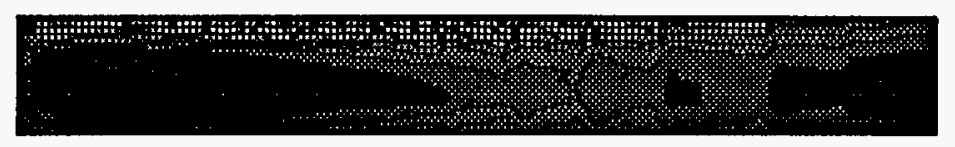

Time $=8.50$ days

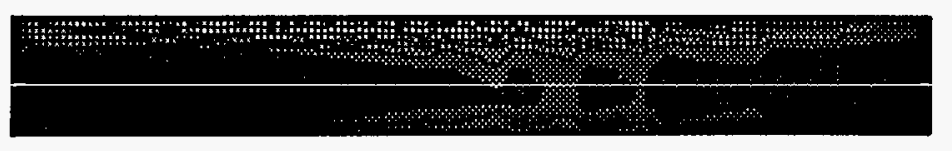

Time $=4.25$ days

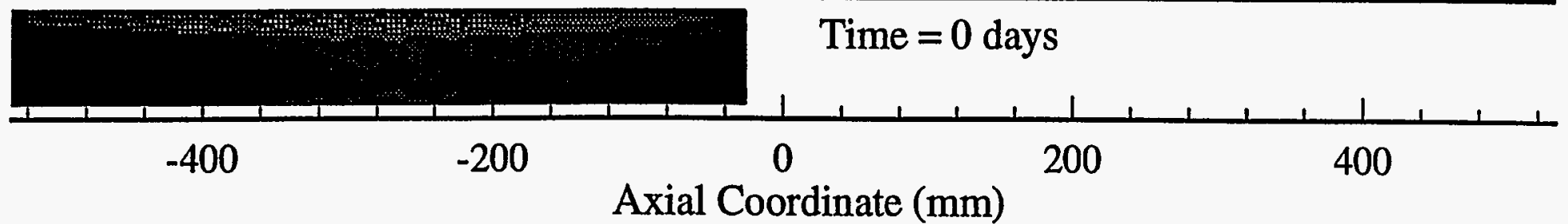

Fig. D.6. Contours of oxide layer thickness, "best estimate" TASHA case, L7 design. 

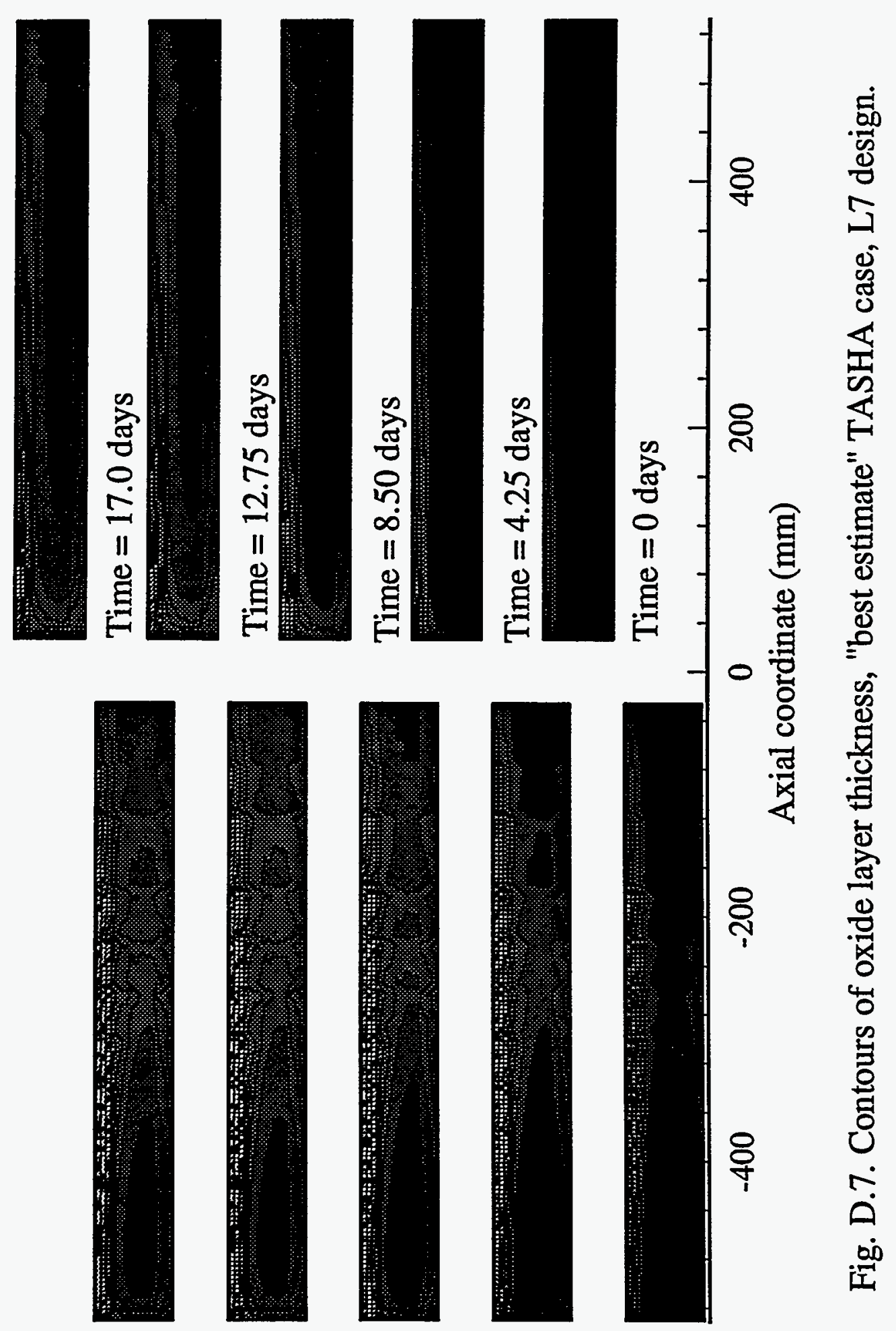

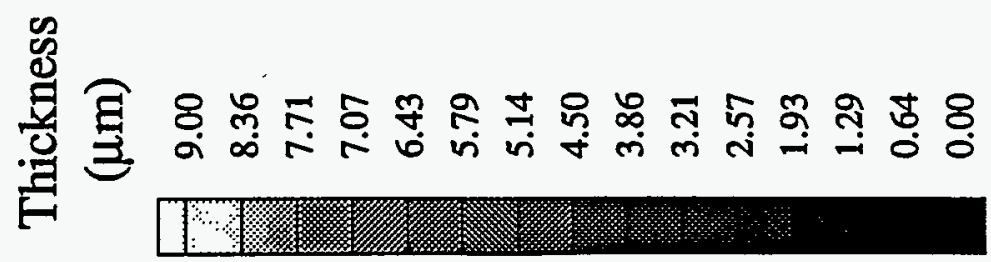


Coolant temperature $\left({ }^{\circ} \mathrm{C}\right)$

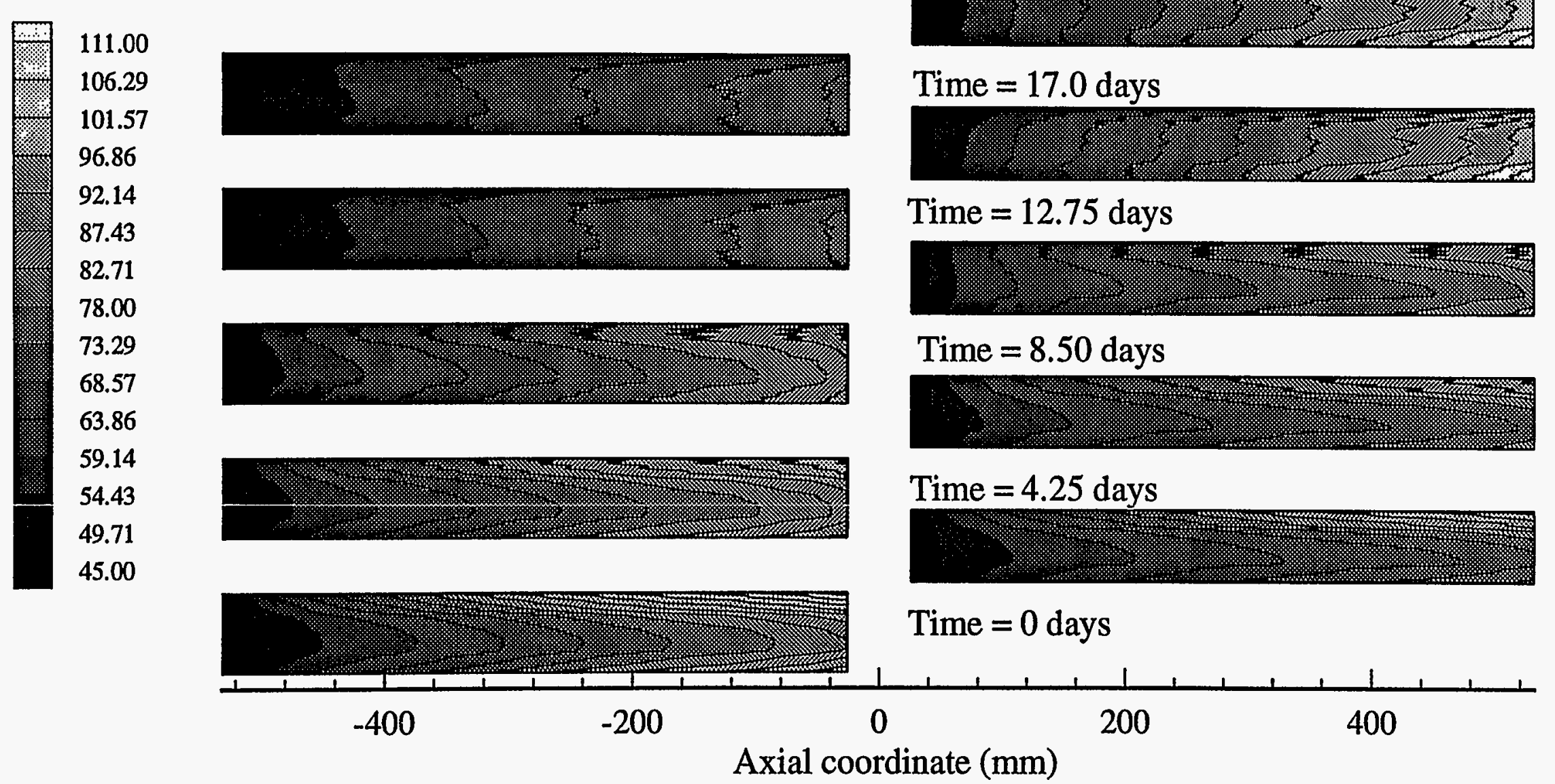

Fig. D.8. Contours of coolant bulk temperature, "best estimate" TASHA case, L7 design. 


\section{D.2 G693 CORE DESIGN FUEL PLATES}

A summary of the data used in the LAT analyses of the G693 core design is presented in the section.

The meat thickness remains constant over the cycle, and the distribution is shown in Fig. D.9. Distributions for both the upper and lower fuel plates are shown in this figure. The thickness colors are the same for both plates.

Figures D.10 to D.15 present the time-dependent data for the power density, thermal conductivities, oxide thickness and coolant temperatures. In these figures, the upper- and lower-plate pair is shown for every time step. The pair of plates for first time step at the bottom of the figure with the pair for the last time step at the top of the figure. The same color spectrum is used for all plates for comparison. These figures show the magnitude of the nonuniformity in both space and time that exist in the ANSR G693 fuel plates. The oxide thickness and coolant temperature distributions for only the "best estimate" TASHA analyses are shown.

Tables D.5 to D.8 present a summary of the time-dependent data of the G693 core design for both the "best estimates" and the $95 \%$ probability cases.

Table D.5. G693 Relative Power and Fission Density

\begin{tabular}{lllll}
\hline $\begin{array}{l}\text { Time } \\
\text { (d) }\end{array}$ & \multicolumn{2}{l}{$\begin{array}{l}\text { Relative power density } \\
\text { Min }\end{array}$} & Max & \multicolumn{2}{l}{ Fission density $\left(\mathrm{f} / \mathrm{mm}^{3}\right)$} \\
\hline 0 & 0.3999 & 1.7197 & 0.0 & Max \\
1.0 & 0.3999 & 1.7197 & $2.249 \mathrm{E}+20$ & 0.0 \\
4.25 & 0.4219 & 1.8102 & $9.548 \mathrm{E}+20$ & $6.978 \mathrm{E}+21$ \\
8.5 & 0.4627 & 1.9014 & $1.945 \mathrm{E}+21$ & $1.266 \mathrm{E}+22$ \\
12.75 & 0.4947 & 1.7306 & $3.036 \mathrm{E}+21$ & $1.742 \mathrm{E}+22$ \\
17 & 0.3006 & 1.7114 & $4.380 \mathrm{E}+21$ & $2.096 \mathrm{E}+22$ \\
\hline
\end{tabular}

Table D.6. G693 Fuel Conductivities

\begin{tabular}{lllll}
\hline \multirow{2}{*}{$\begin{array}{l}\text { Time } \\
\text { (d) }\end{array}$} & Normal fuel & \multicolumn{3}{c}{ Thermal Conductivity $\left(\mathrm{W} / \mathrm{cm}^{\circ} \mathrm{C}\right)$} \\
& Min & Max & Min & Max \\
\hline 0 & 1.650 & 1.650 & 0.145 & 0.145 \\
1.0 & 1.650 & 1.650 & 0.145 & 0.145 \\
4.25 & 1.651 & 1.659 & 0.115 & 0.134 \\
8.5 & 1.561 & 1.707 & 0.122 & 0.350 \\
12.75 & 1.436 & 1.707 & 0.141 & 0.547 \\
17 & 1.228 & 1.707 & 0.197 & 0.547 \\
\hline
\end{tabular}


Table D.7.

G693 TASHA 95\% Limits Case Oxide Thickness and Coolant Temperature

\begin{tabular}{lllll}
\hline $\begin{array}{l}\text { Time } \\
(\mathrm{d})\end{array}$ & \multicolumn{2}{l}{ Oxide layer thickness $(\mu \mathrm{m})$} & \multicolumn{2}{l}{ Coolant temperature $\left({ }^{\circ} \mathrm{C}\right)$} \\
& Min & $\operatorname{Max}$ & Min & Max \\
\hline 0 & 0.0 & 0.0 & 45.628 & 110.50 \\
1.0 & 0.1467 & 2.16490 & 45.628 & 110.52 \\
4.25 & 0.4679 & 6.08270 & 45.628 & 109.52 \\
8.5 & 0.8035 & 8.26080 & 45.628 & 105.28 \\
12.75 & 1.1804 & 9.37100 & 45.628 & 103.08 \\
17 & 1.9579 & 10.19880 & 45.628 & 114.91 \\
\hline
\end{tabular}

Table D.8.

G693 TASHA "best estimates" Case Oxide Thickness and Coolant Temperature

\begin{tabular}{|c|c|c|c|c|}
\hline \multirow{2}{*}{$\begin{array}{l}\text { Time } \\
\text { (d) }\end{array}$} & \multicolumn{2}{|c|}{ Oxide layer thickness $(\mu \mathrm{m})$} & \multicolumn{2}{|c|}{ Coolant temperature $\left({ }^{\circ} \mathrm{C}\right)$} \\
\hline & Min & $\operatorname{Max}$ & Min & $\operatorname{Max}$ \\
\hline 0 & 0.0 & 0.0 & 45.0 & 99.994 \\
\hline 1.0 & 0.062 & 0.627 & 45.0 & 100.01 \\
\hline 4.25 & 0.208 & 1.780 & 45.0 & 99.147 \\
\hline 8.5 & 0.355 & 2.566 & 45.0 & 95.535 \\
\hline 12.75 & 0.513 & 2.938 & 45.0 & 93.663 \\
\hline 17 & 0.805 & 3.146 & 45.0 & 77.688 \\
\hline
\end{tabular}




\section{D.13}

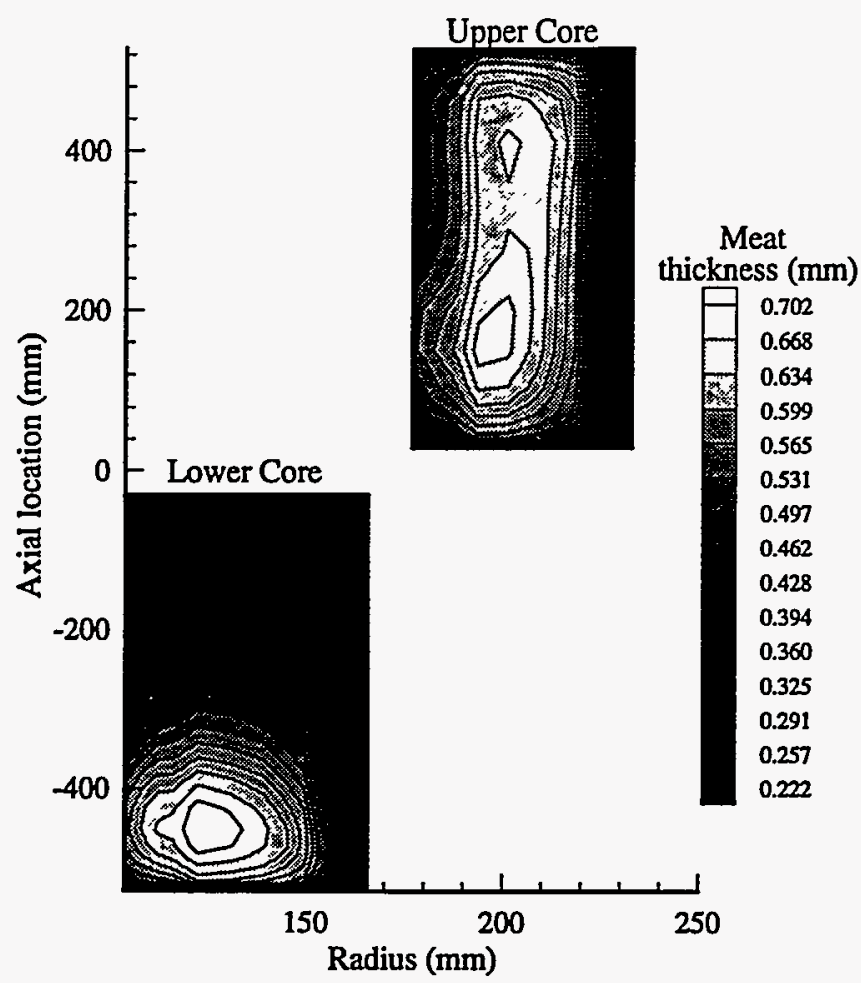

Fig. D.9. Meat thickness distribution for the L7 core design. 
Relative power

Density

1.80

1.70

1.59

1.49

1.39

1.28

1.18

1.07

0.97

0.87

0.76

0.66

0.56

0.45

0.35
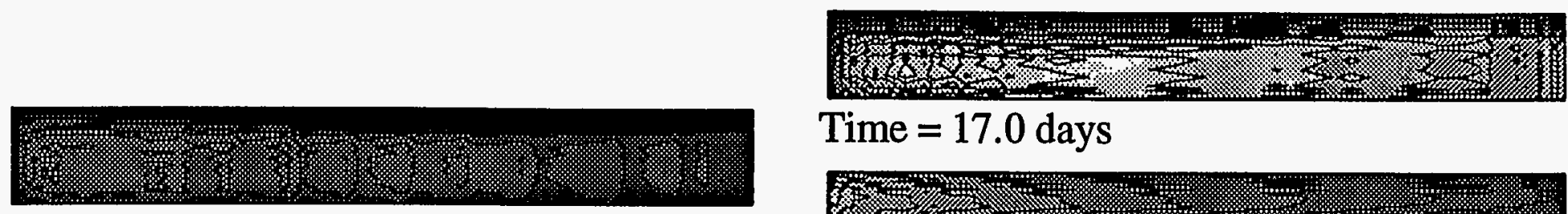

Time $=17.0$ days
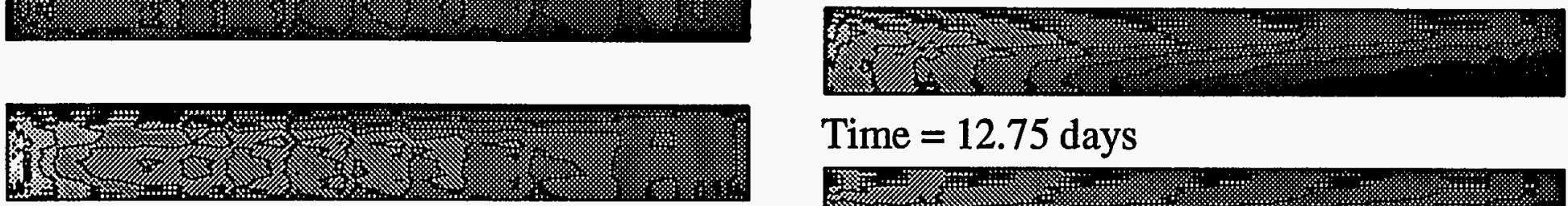

Time $=12.75$ days
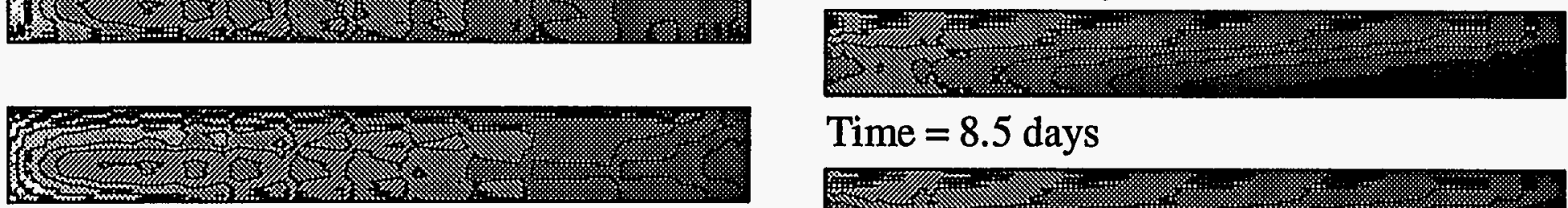

Time $=8.5$ days
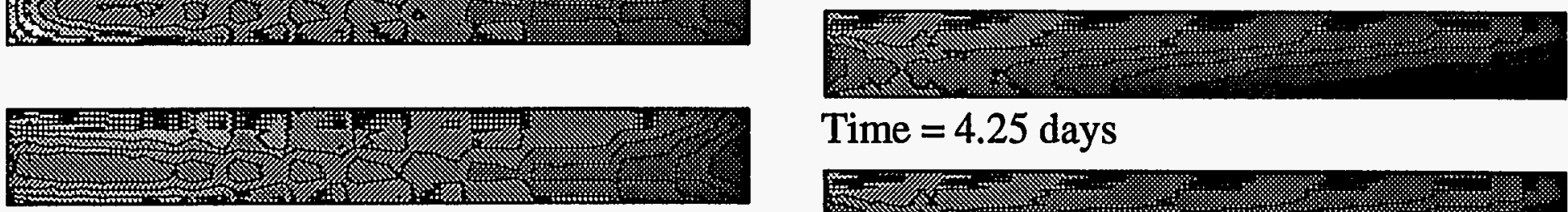

Time $=4.25$ days
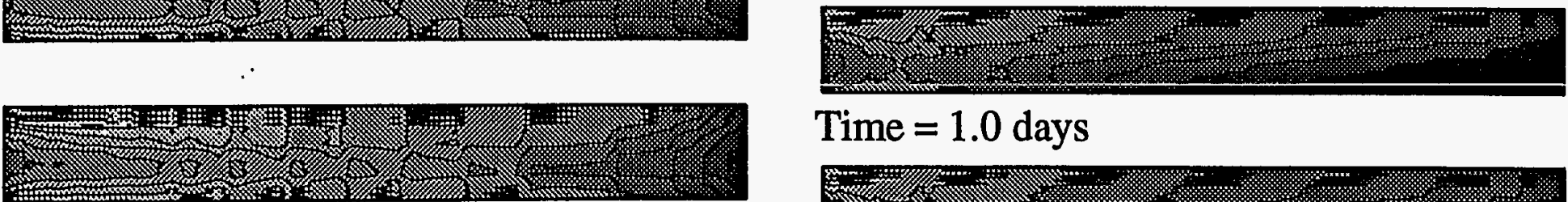

Time $=1.0$ days

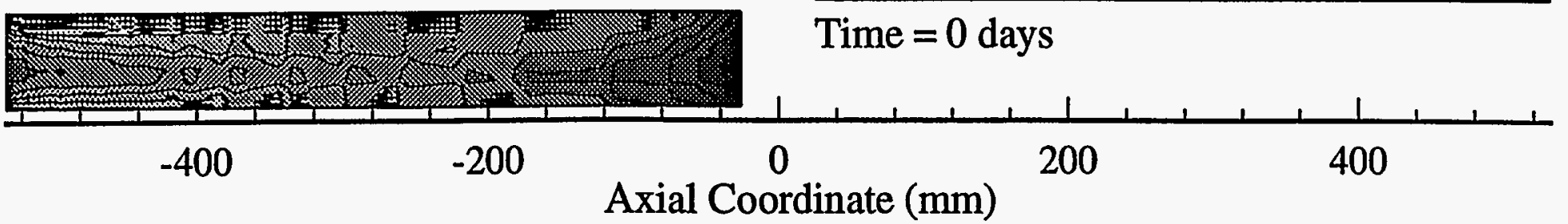

Fig. D.10. Contours of relative power density for each time step, G693. 


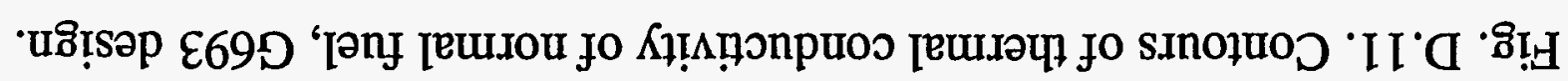

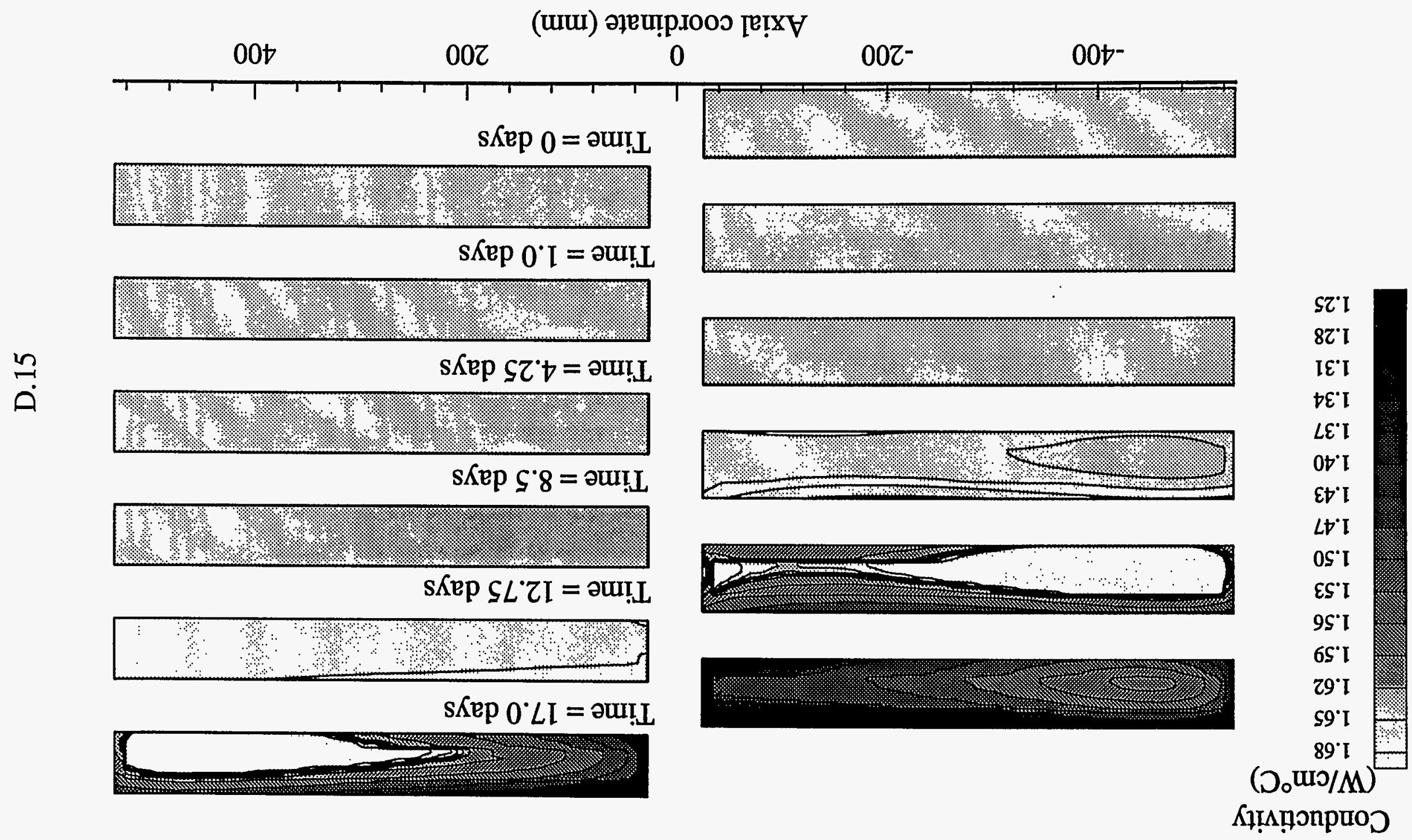




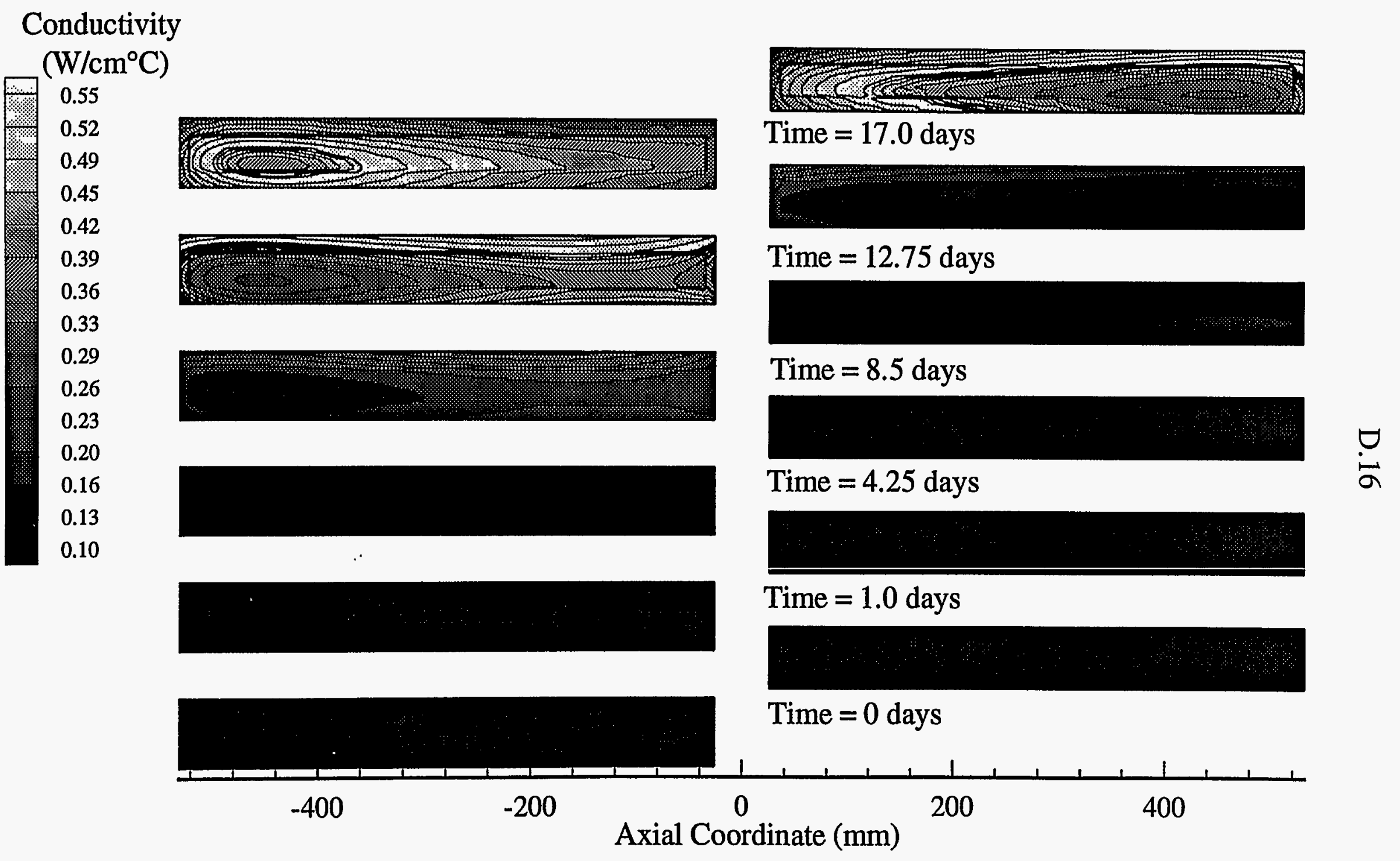

Fig. D.12. Contours of thermal conductivity of segregated fuel, G693. 

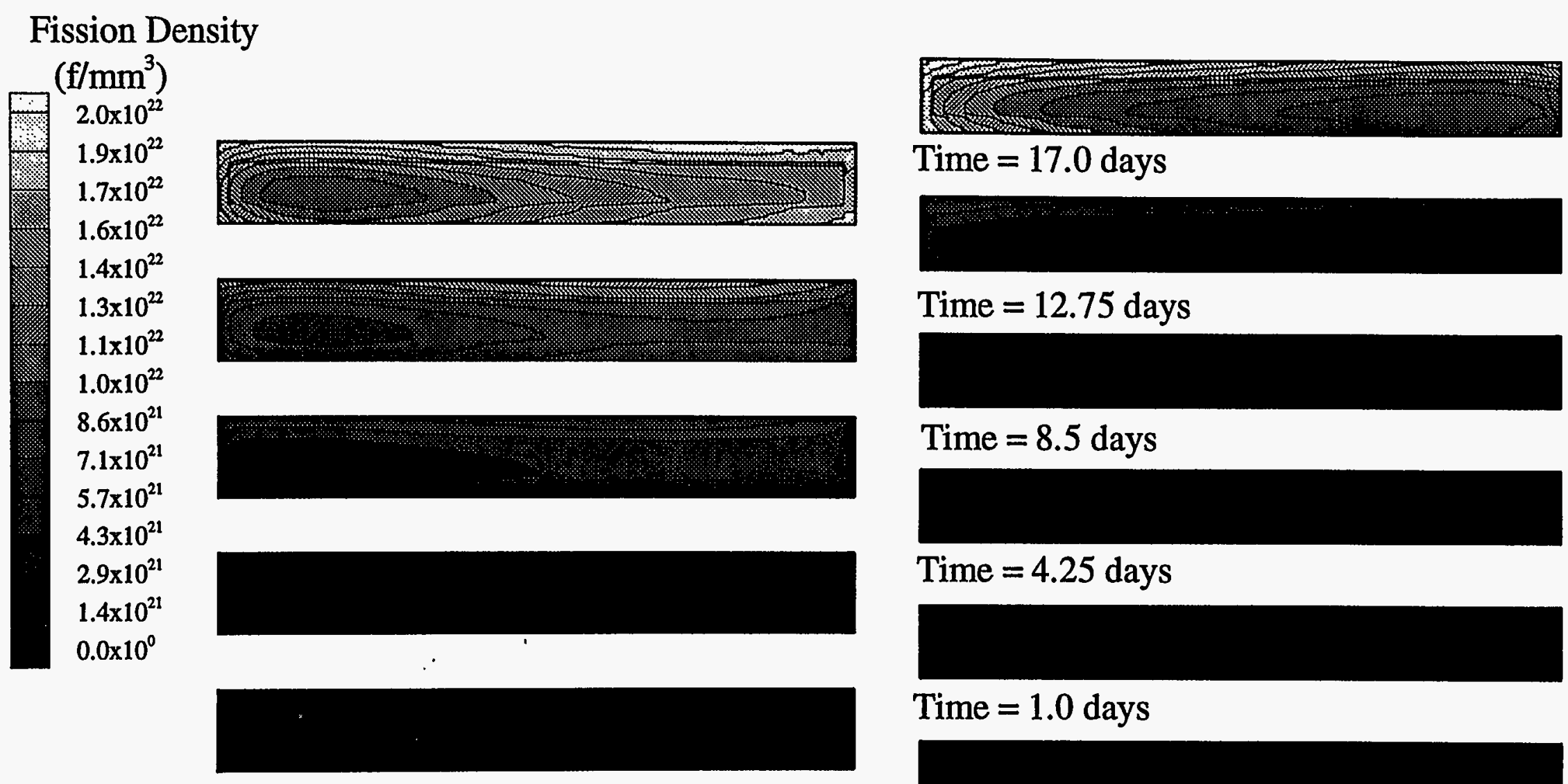

Time $=17.0$ days

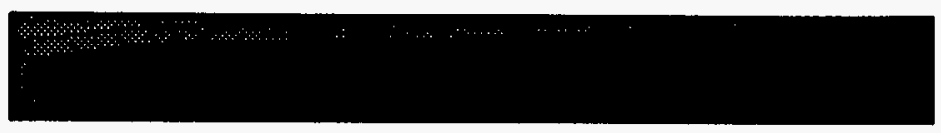

Time $=12.75$ days

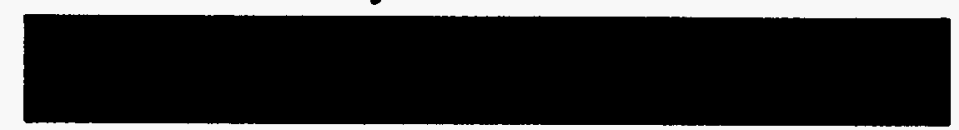

Time $=8.5$ days

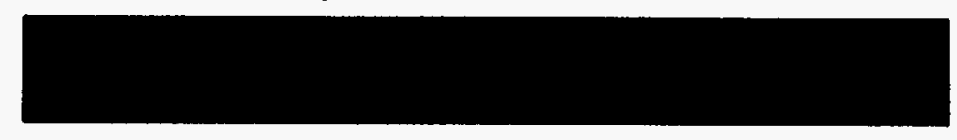

Time $=4.25$ days

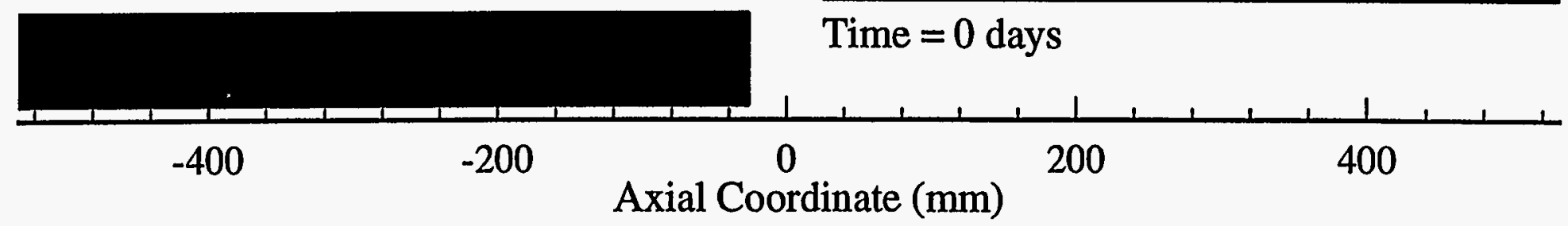

Fig. D.13. Contours of fission density, G693. 
Thickness ( $\mu \mathrm{m})$

a 3.00

2.79

2.57

2.36

2.14

1.93

1.71

1.50

1.29

1.07

0.86

0.64

0.43

0.21

0.00

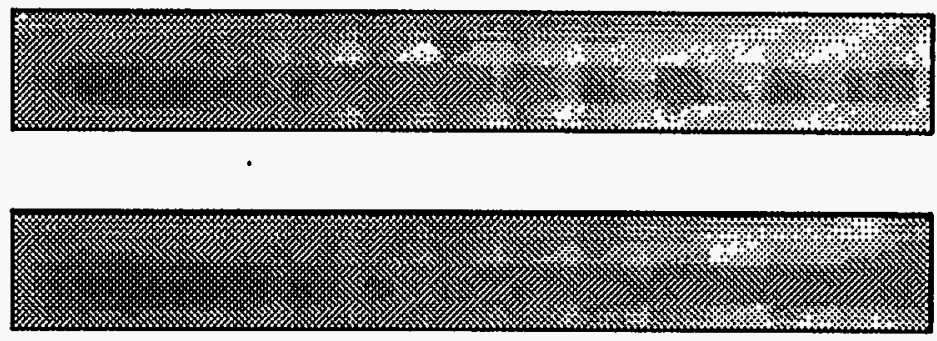

Time $=17.0$ days

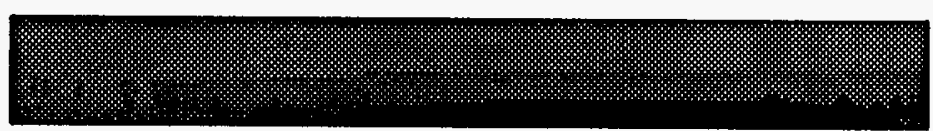

Time $=12.75$ days

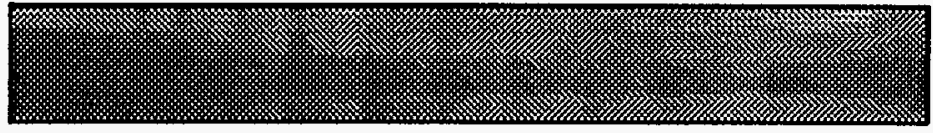

Time $=8.5$ days

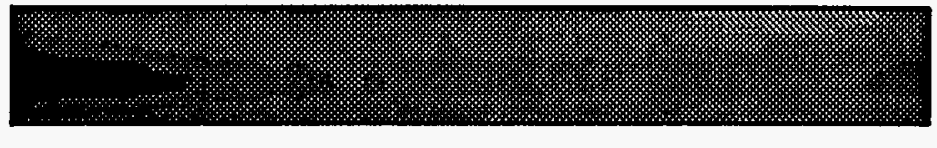

Time $=4.25$ days
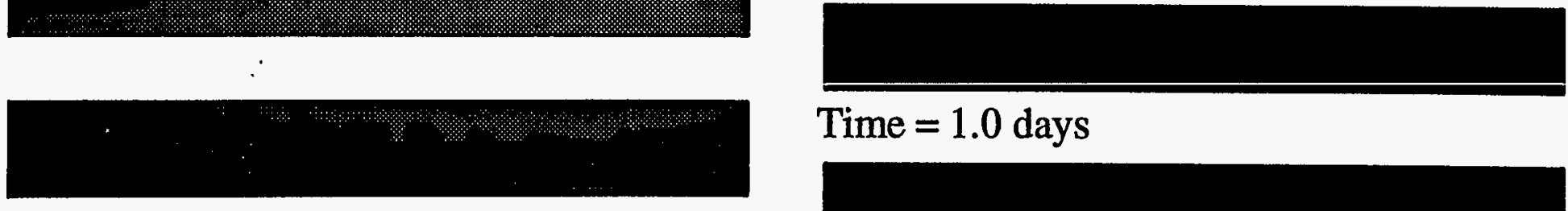

Time $=1.0$ days

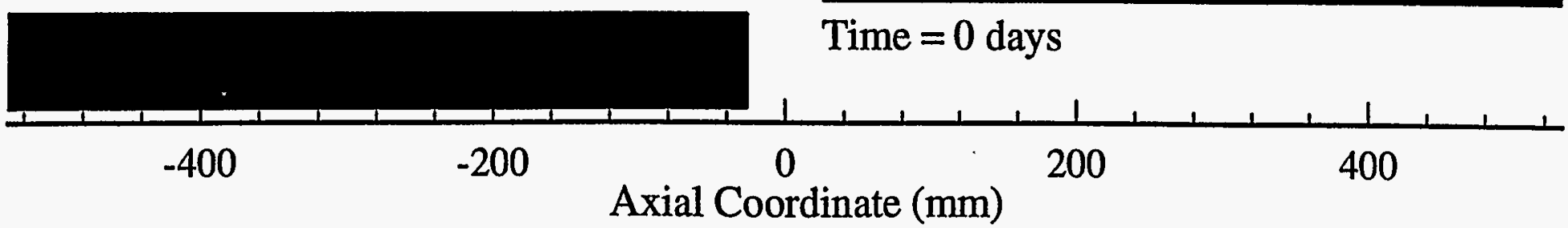

Fig. D.14. Contours of oxide layer thickness, "best estimate" TASHA case, G693. 
Temperature $\left({ }^{\circ} \mathrm{C}\right)$

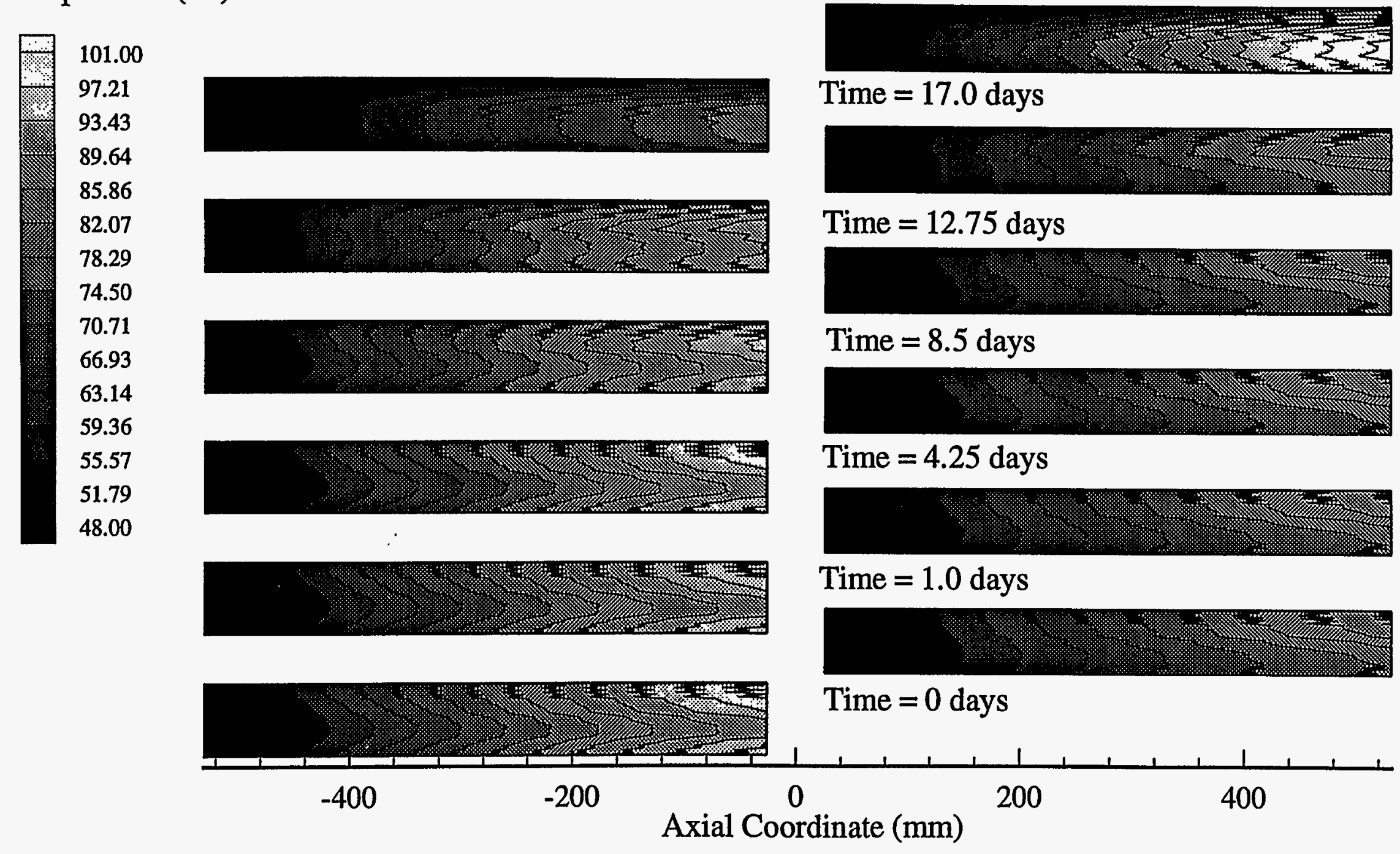

Fig. D.15. Contours of coolant temperature,"best estimate" TASHA case, G693. 


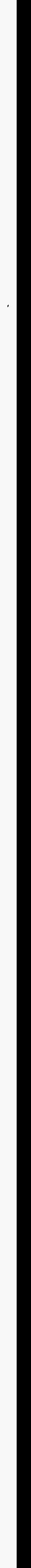




\section{Appendix E}

\section{LOCAL TIME-DEPENDENT FISSION DENSITY}

\section{E.1 METHOD OF CALCULATION}

The local hot spot thermal analysis requires the local fission density history for each point on the fuel plate. The VENTURE code supplies the local burnup only at the end of the cycle. However, the local fission density history can be calculated from the power density history which is reported from the VENTURE code. The following are the steps used to calculated the local fission density history.

The fission density is directly related to the power density since each fissioned atom generates a known amount of energy. The energy per $235 \mathrm{U}$ fission is $202 \times 10^{6} \mathrm{ev}$ or $3.24 \times 10^{-11} \mathrm{~J}$. The fission density for each time step is calculated from the integral of the power density as:

$$
\mathrm{Fd}_{\mathrm{i}+1}=\mathrm{Fd}_{\mathrm{i}}+\left(\frac{\left(\mathrm{Pd}_{\mathrm{i}+1}+\mathrm{Pd}_{\mathrm{i}}\right)}{2}\right) \mathrm{Fd}_{\mathrm{a}} \Delta \mathrm{t}_{\mathrm{i}} \frac{\mathrm{m}_{\mathrm{a}}}{\mathrm{m}_{\mathrm{x}}},
$$

where

$$
\begin{aligned}
\mathrm{Pd} & =\text { local relative power density, } \\
\Delta \mathrm{t} & =\text { time step size, } \mathrm{s} \\
\mathrm{m}_{\mathrm{a}} & =\text { average fuel meat thickness, } \mathrm{mm}, \\
\mathrm{m}_{\mathrm{x}} & =\text { maximum fuel meat thickness, } \mathrm{mm}, \\
\mathrm{Fd}_{\mathrm{a}} & =\text { average fission rate density in fuel, } \frac{\text { fissions }}{\left(\mathrm{s} \mathrm{\textrm {mm } ^ { 3 } )}\right.} \\
& =\frac{\mathrm{C}_{\mathrm{p}}}{\mathrm{V}_{\mathrm{f}} \mathrm{e}_{\mathrm{f}}}=6.953 \times 10^{12} \\
\mathrm{C}_{\mathrm{p}} & =\text { core power, } 332 \mathrm{MW} \\
\mathrm{V}_{\mathrm{f}} & =\text { volume of } \mathrm{U}_{3} \mathrm{Si}_{2}, 1.47534 \times 10^{6} \mathrm{~mm}^{3}, \\
& =\frac{\mathrm{M}_{\mathrm{f}}}{\text { En } \mathrm{R}_{\text {uus }} \rho_{\mathrm{us}}} \\
\mathrm{M}_{\mathrm{f}} & =\text { fuel mass, } 15.45 \mathrm{~kg}, \\
\mathrm{En} & =\text { enrichment, } 0.932 \\
\mathrm{Ruus} & =\text { ratio of } \mathrm{U} \text { in } \mathrm{U} 3 \mathrm{Si} 2,0.921 \\
\rho_{\mathrm{us}} & =\text { density of } \mathrm{U}_{3} \mathrm{Si}_{2}, 0.0122 \mathrm{~g} / \mathrm{mm}^{3} \\
\mathrm{e}_{\mathrm{f}} & =\text { energy per fission, } \mathrm{J} .
\end{aligned}
$$


The power density averaging over time is consistent with the VENTURE code method of calculating the burnup.

Although the VENTURE code does not report these intermediate results, the end-of-cycle (EOC) burnup is reported. The EOC burnup data can be used to calculate the EOC fission density, $F_{d e}$, for comparison with the fission density calculated from the power density.

$$
F d_{e}=\frac{S_{d} C_{r} B_{u}}{m F_{v}}\left(\frac{A_{v}}{M_{w}}\right) \frac{\text { fissions }}{m m^{3}}
$$

where

$$
\begin{aligned}
\mathrm{S}_{\mathrm{d}} & =\text { initial } 235 \mathrm{U} \text { surface density, }\left(\mathrm{g} / \mathrm{mm}^{2}\right) \\
\mathrm{m} & =\text { meat thickness, mm, } \\
\mathrm{F}_{\mathrm{v}} & =\text { volume fraction of fuel, } \\
& =0.124 \frac{\mathrm{U}_{3} \mathrm{Si}_{2} \text { volume }}{\text { meat volume }} \text { for } \mathrm{G} 693 \text { core design, } \\
\mathrm{A}_{\mathrm{v}} & \left.=\text { Avogadro's number, } 6.023 \times 10^{23} \text { (molecules } / \mathrm{g}-\mathrm{mol}\right), \\
\mathrm{M}_{\mathrm{w}} & =\text { molecular weight, } 235(\mathrm{~g} / \mathrm{g}-\mathrm{mol}) . \\
\mathrm{B}_{\mathrm{u}} & =\text { burnup at EOC, } \rho_{\mathrm{uf}} / \rho_{\mathrm{ua}} \\
\rho_{\mathrm{uf}} & =\text { density of fissioned } 235 \mathrm{U} \\
\rho_{\mathrm{ua}} & =\text { maximum density of } 235 \mathrm{U} \text { atoms that can fission, } \\
& =\mathrm{C}_{\mathrm{r}} \text { (235U atom density) }=\mathrm{C}_{\mathrm{r}} \frac{\mathrm{S}_{\mathrm{d}}}{\mathrm{m}} \\
\mathrm{C}_{\mathrm{r}} & =\text { capture ratio, number of fissions per absorbed neutrons, } 0.84 .
\end{aligned}
$$

The VENTURE code calculates the burnup by a similar process and then uses a cell-averaging technique to report the data on the coarse grid used for the meat thickness distribution. Figure E.1 presents a comparison of the two sets of distributions at EOC. The shaded contours are taken from the power-density-generated fission density while the thick line contours are from the burnup-data-generated fission density (VENTURE results). The general shape and location of the contours appear reasonable.

Figure E.2 presents the relative difference between the two sets of distributions. In general the two distributions match very well over most of the surface of the plate. In the area of the maximum fission density, the power-density-generated fission density exceeds the VENTURE results by at most $12 \%$. This is reasonable since the coarse grid cell-averaging technique would tend to reduce the fission density in the area of these steep gradients. In the area of the minimum fission density, the power-density-generated fission density is lower than the VENTURE results by at most $-14 \%$. Some of this difference is attributable to the coarse grid 
and cell-averaging technique used to report the burnup data. However, the shallow gradients in these areas make this argument less convincing.

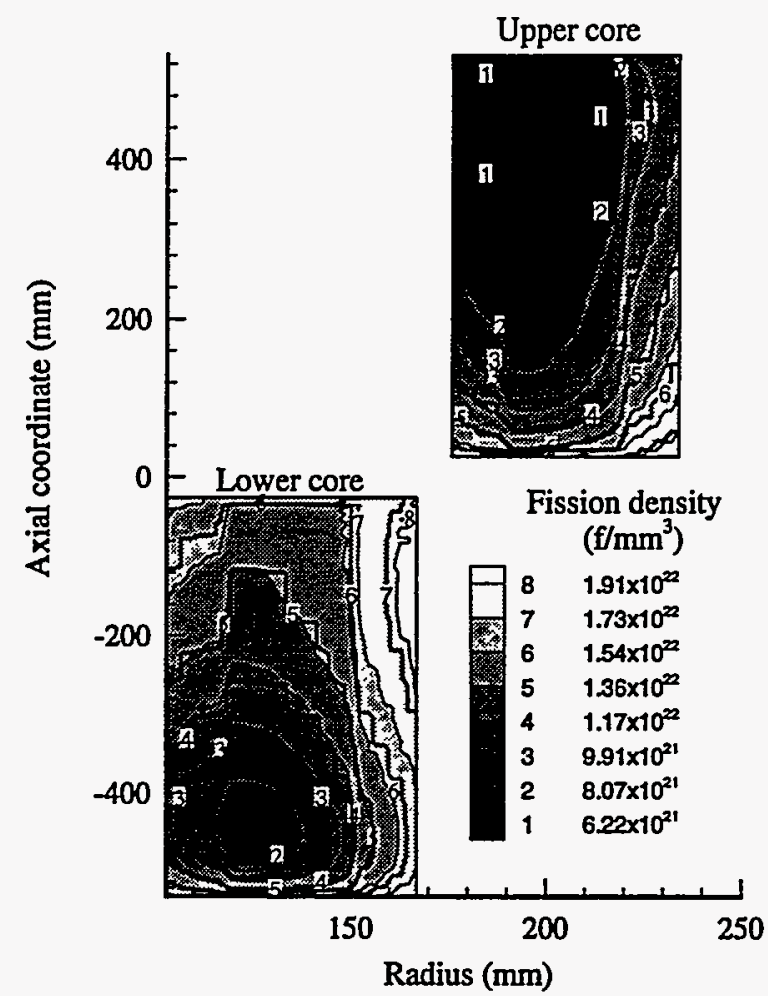

Fig. E.1. Comparison of fission density generated from power density (shaded) and burnup data (thick lines).

\section{E.2 GENERATION OF FISSION DENSITY DATA}

The Table E. 1 contains the listing of the computer code MK-FD. This version was used for the G693 core design. The code uses as input the VENTURE input meat thickness distribution data set and VENTURE output relative power density data set that are described in Appendix A. MK-FD generates a file containing the fission density as a function of time. The format of this file is described in Table E.2.

As a check on the integration process, the VENTURE produce surface density distribution data set and the EOC burnup distribution data set can be used to calculate the EOC fission density. The formats of the surface density and burnup distributions are described in Tables. E.3 and E.4. The EOC burnup and fission density data from both methods of calculation are output in a format that can be used in the TECPLOT to generate the figures above. The format of this file is described in Table E.5. 


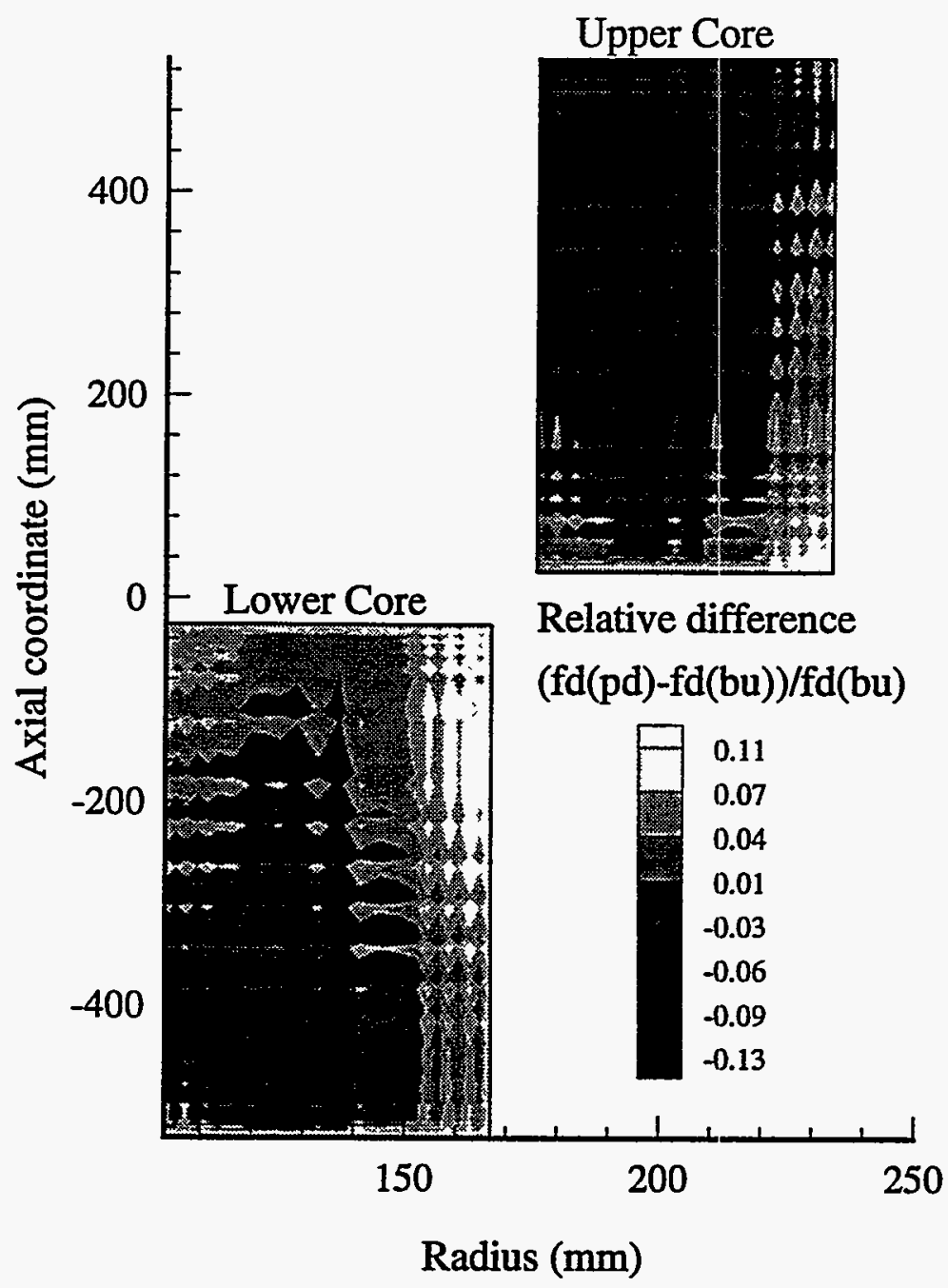

Fig. E.2. Relative difference between fission density generated from power density and burnup data. 


\section{Table E.1. Listing of MK-FD.F computer code}

c

C

c

c

C

C

c

c

c

c

c

C

c

c

c

this code read the pd tables and generates the fd tables.

the $f d$ is generated on the pd grid

parameter (maxpds $=4000, \operatorname{maxp} 2=1000)$

dimension $r$ (maxpds), $z$ (maxpds), $r c(\operatorname{maxp} 2), d r(\operatorname{maxp} 2), z c(\operatorname{maxp} 2)$, tm (maxp2), pd (maxpds) , pdc (maxp2), dz (maxp2), sd (maxp2) , cd (maxp2) , oxc (maxp2) , cdr $(\operatorname{maxp} 2)$, oxu $(50,80), \operatorname{oxl}(50,80)$, ox (maxpds), rou (maxpds), rol (maxpds), zou (maxpds), zol (maxpds), nr (maxpds), $\mathrm{nz}$ (maxpds)

dimension fd(maxpds), bueoc (maxpds), diff (maxpds), fdtim(7), $\operatorname{pdm}(8), f d m(8)$

$\&$

character cline $* 80,1$ abel, equal

data zero $/ 0.0 /$, tmave $/ 0.4466 /, \operatorname{xnfn} / 0.84 /$,

\& fatim/ $0,1.0,4.25,8.5,12.75,17.0,21.25 /$, iend $/ 0 /$,

\& ioptsd/1/,ipmx/1518/

iend $=0$ eoc $=$ last power density data set

iend $=1$ eoc $=$ last $\mathrm{pd}+$ delta-days

volf $=1.507 / 12.2$

open data sets

if (ioptsd.eq.1) open (unit=3, file='g693-sd',

$\&$ status $=$ 'old')

if (ioptsd.eq.1) open (unit $=4$, file='g693-bu', status='old')

open (unit $=5$, file $={ }^{\prime} . . / g 693-t m^{\prime}$, status='old')

open (unit=7, file='g6t1-6fd' , status='new')

open (unit=2,file=' . /g693p1-6', status='old')

open (unit $=6$, file $=$ ' omkfd', status $=$ 'new' )

open (unit $=10$, file=' tecmkfd', status =' new' ) write header for contours

write $(10, *)$ 'title=' 'mk-fd $x y$ '' '

write $(10, *)$ 'variables $=r z$ bupd fdeoc fd dif'

write $(10, *)$ 'zone

read the surface density and meat thickness table

read file that has $r, z$, and tm

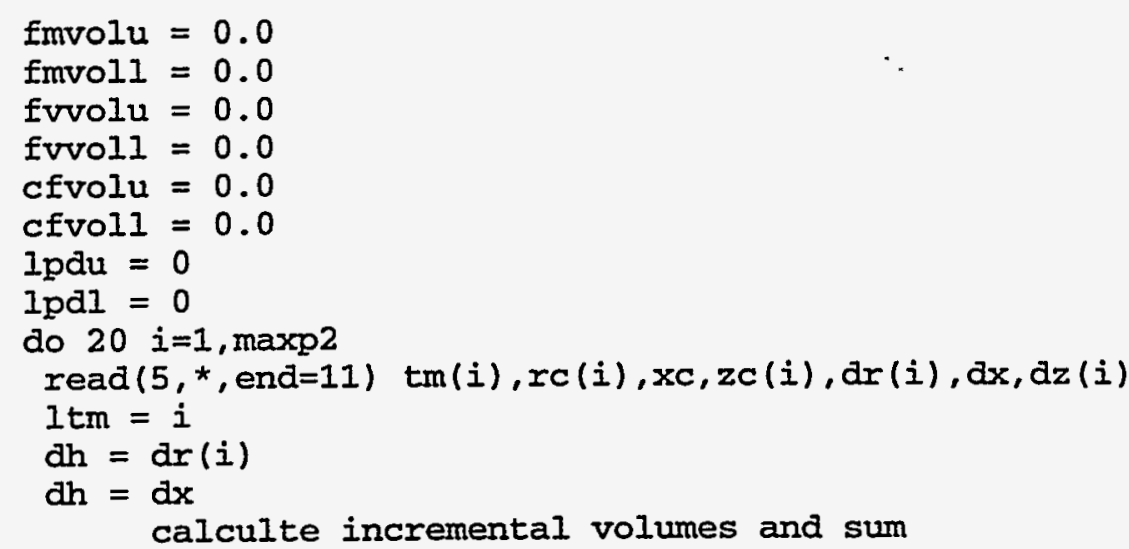


Table E.1. Listing of MK-FD.F computer code (continued)

c

call calvis (fmvolu, fmvoll, fvvolu, fvvoll, cfvolu, cfvoll, $\&$ if(ioptsd.eq.1) then $d h, z c(i), d z(i), \operatorname{tm}(i))$ read file that has surface density data and check to see if in the same order as the tm data set

$\operatorname{read}(3, *, e n d=11) \mathrm{sdo}, \mathrm{rc0}, \mathrm{xc}, \mathrm{zc0}$

if(dist (rc(i), zc(i), rc0, zc0).1e.1.0d-5) then $\operatorname{sd}(i)=s d 0$

else

write $(6, *)$ ' sd \& tm tables different order'

stop

endif

endif

continue

continue

output volume info

call wrtvol (fmvolu, fmvoll, fvvolu, fvvoll, cfvolu, cfvoll)

iupmid $=1$

ilpmid $=1$

iupend $=1$

ilpend $=1$

calculate fd and write out in data set

the power density for time step 1 is used to calculate

the fission density at time step 2

do 100 it $=1,6$

fdemax $=0$

fdemin $=1 d 30$

iffit.eq.1) then

write $(7, *)$ 'TIME= ', fatim(1)

do $30 i w=1,1 p d$

$f d(i w)=0.0$

continue

else

time steps $2-5$

read the next power density time step

call rapd (pdtim, pd, lpd, ltm, r, z, rc, zc, pdc, dr, dz)

if(it.eq.2) then

$$
\text { do } 31 \text { iw=1, Ipd }
$$

write out fd for first time step'need to know Ipd write $(7, *) r(i w), z(i w), f d(i w)$

$\&$ call pamid (lpd, r, $z$, rumid, zumid, rlmid, zlmid, iupmid, ilpmid,

endif iupend, ilpend)

time difference in seconds deltat $=(\operatorname{fatim}(i t)-\operatorname{fdtim}(i t-1)) * 24 * 3600$ write $(7, *)$ 'TIME $=$ ', $\operatorname{fdtim}(i t)$

calculate the local fd from the current power density mass of fuel $\mathrm{U} 235=15.45 \mathrm{~kg}$ volume of fuel particles $=1.475 \mathrm{e} 6 \mathrm{~mm}^{\wedge} 3=1.4751$ 


\title{
Table E.1. Listing of MK-FD.F computer code (continued)
}

c

C

C

C

C

c

C

C

C

C

C

c

\author{
power $=332 \mathrm{MW}$ \\ $202 \mathrm{Mev} / \mathrm{fission}$ \\ $1.60219 e-19 j / e v$ \\ $j /$ fission $=3.236 \mathrm{e}-11$
}

confac $=$ average fission rate in fuel particles confac is the number of fissions/scu. [mc]m at relative power density $(\mathrm{pd})=1.0$ for fuel particle confac $=$ power $/($ (vol fuel) $*$ joules/fiss)

confac $=6.953 \mathrm{el}$ fission/ (s $\mathrm{Im}^{\wedge} 3$ ) confac $=6.953 \mathrm{e} 15$ fission $/\left(\mathrm{s} \mathrm{cm}^{\wedge} 3\right)$

pd = relative power density $\left(W / \mathrm{cm}^{\wedge} 2\right) /\left(W / \mathrm{cm}^{\wedge} 2\right)$ ave to convert to heat generation rate * (tmave/tm)

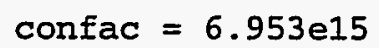

difmax $=0.0$

relmax $=0.0$

relave $=0.0$

difave $=0.0$

$i d \max =1$ 


\section{E.8}

\section{Table E.1. Listing of MK-FD.F computer code (continued)}

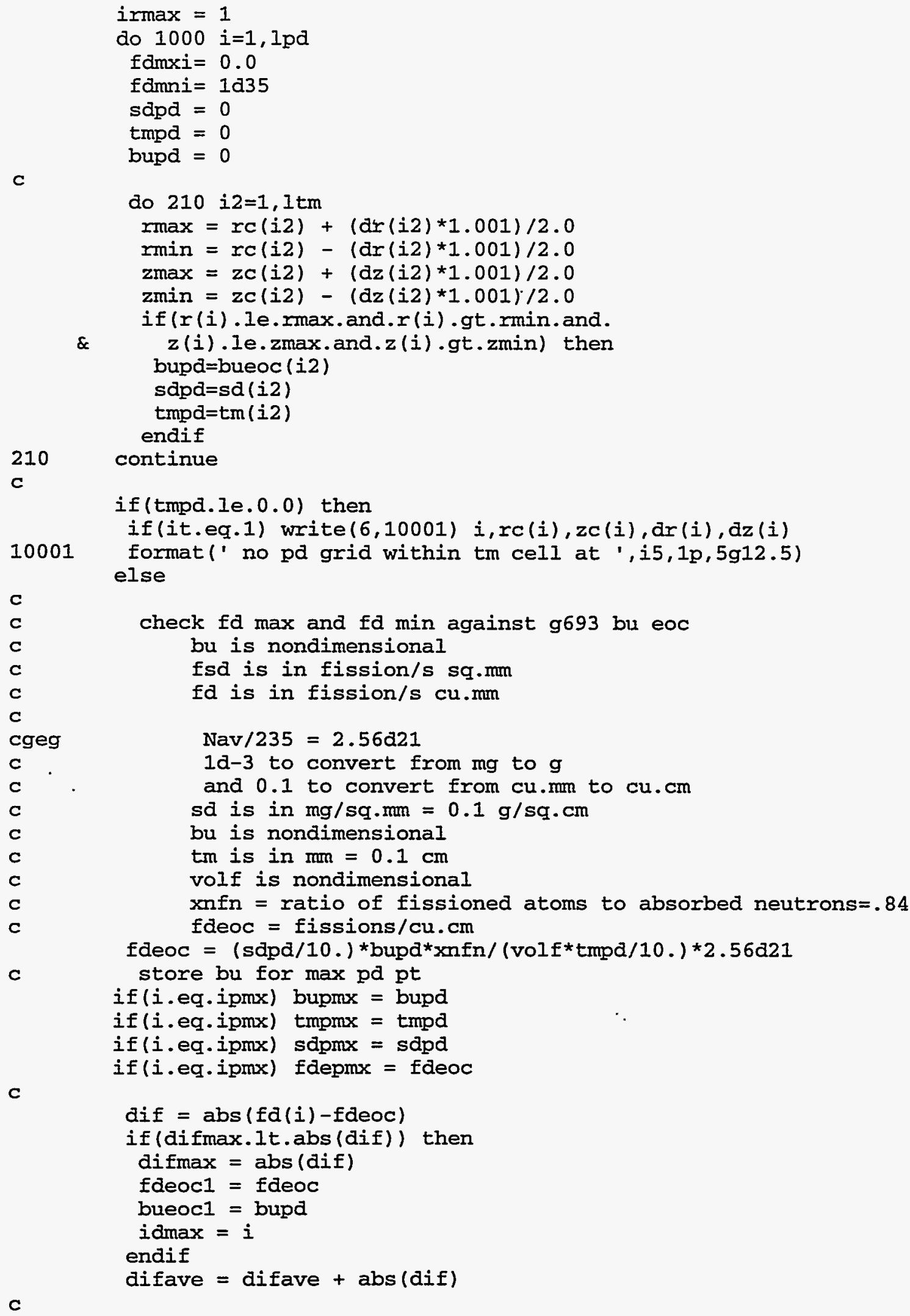

C 
Table E.1. Listing of MK-FD.F computer code (continued)

C

C

C

c output in tecplot format

write $(10, *) r(i), z(i)$, bupd, fdeoc, fd(i), dif endif

1000 continue

c

$\&$

C

\&

write $(6$,

'(/, ' r $r, z, p d, b u, s d, t m, f d e o c, f d e=', /, i 5,1 p, 8 g 12.5)$ ') ipmx,

\& $r(i p m x), z(i p m x), p d$ (ipmx), bupmx, sdpmx, tmpmx, fdepmx, fd (ipmx)

write (6, ' (/,' ' fdeocmax, at, fdeocmin, at' ',2(1p,g12,5, 0p, i5))')

C

difave = difave/lpd

relave $=$ relave $/ 1 \mathrm{pd}$

write $(6, *)$, '

write $(6, *)$ ' Maximum diff between fd at eoc=', difmax

write $\left(6,{ }^{*}\right)$ ' idmax, $r, z$, bueoc, fdeoc, fddmx' ,

$\&$ 


\section{E.10}

Table E.1. Listing of MK-FD.F computer code (continued)

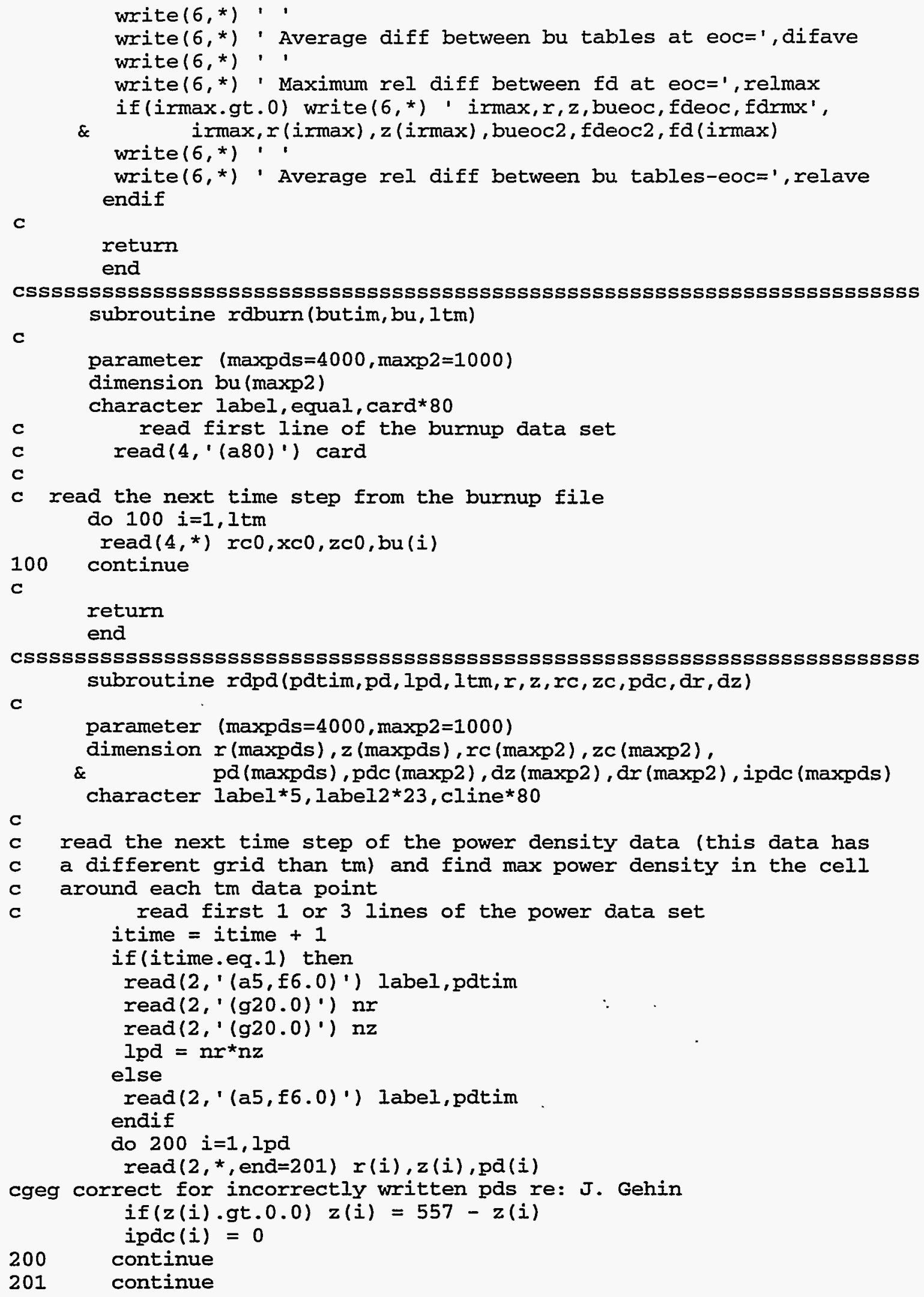




\section{Table E.1. Listing of MK-FD.F computer code (continued)}

C

c

$t$ mrmn $=1 a 5$
$t$ mzmn $=1 \mathrm{d5}$
tmrmx $=0.0$
tmrmx $=0.0$

do $1000 \quad i=1,1 \mathrm{tm}$

search for the maximum pd in the grid block defined by the tm table

$\operatorname{rmax}=r c(i)+(\mathrm{dr}(i) * 1.001) / 2.0$

$\operatorname{rmin}=r c(i)-(\mathrm{dr}(i) * 1.001) / 2.0$

$z \max =z c(i)+(\mathrm{dz}(i) * 1.001) / 2.0$

$z \min =z c(i)-(\mathrm{dz}(i) * 1.001) / 2.0$

c

$$
\begin{aligned}
& \text { tmrmx }=\max (t m r m x, \text { rmax }) \\
& \text { tmrmn }=\min (t m r m n, r m i n) \\
& \text { tmzmx }=\max (t m z m x, z \max ) \\
& \text { tmzmn }=\min (t m z m n, z m i n)
\end{aligned}
$$

c

pdmaxi $=0.0$

$$
\begin{aligned}
& \text { do } 300 \text { i2 }=1 \text {, lpd } \\
& \text { if }(x(i 2) \text {.le.rmax. and.r(i2).gt.rmin.and. }
\end{aligned}
$$

$\varepsilon$ $z$ (i2).le.zmax.and.z(i2).gt.zmin) then pdmaxi $=\max (p d \max i, p d(i 2))$

$$
\operatorname{ipdc}(i 2)=1
$$

endif

continue

$\operatorname{pdc}(i)=\operatorname{pdmaxi}$

C

if (pdmaxi.le.0.0) then

imin $=0$

dmin $=1 \mathrm{~d} 4$

do 310 i2 $=1$, lpd

$d=\operatorname{dist}(r(i 2), z(i 2), r c(i), z c(i))$

if (dmin.gt.d) then

$\mathrm{dmin}=\mathrm{d}$

imin $=i 2$

endif

310

continue

if(imin.gt.0) then

$\operatorname{pdc}(i)=p d(i m i n)$

write $(6,10001) i, r c(i), z c(i), d r(i), d z(i)$

10001 format (' no pd grid within tm cell at ',i5,1p,5g12.5)

else

write $(6,10000) i, \operatorname{rc}(i), z c(i), d r(i), d z(i)$

10000

format $(i 5,1 p, 5 \mathrm{~g} 12.5)$

endif

endif

1000 continue

c

if(itime.le.0) then

itime $=1$

C

c

find maximum $r$ and $z$ in upper and lower core from pd data

rmxpdu $=0$

zmxpdu $=-1 d 5$ 


\section{E.12}

\section{Table E.1. Listing of MK-FD.F computer code (continued)}

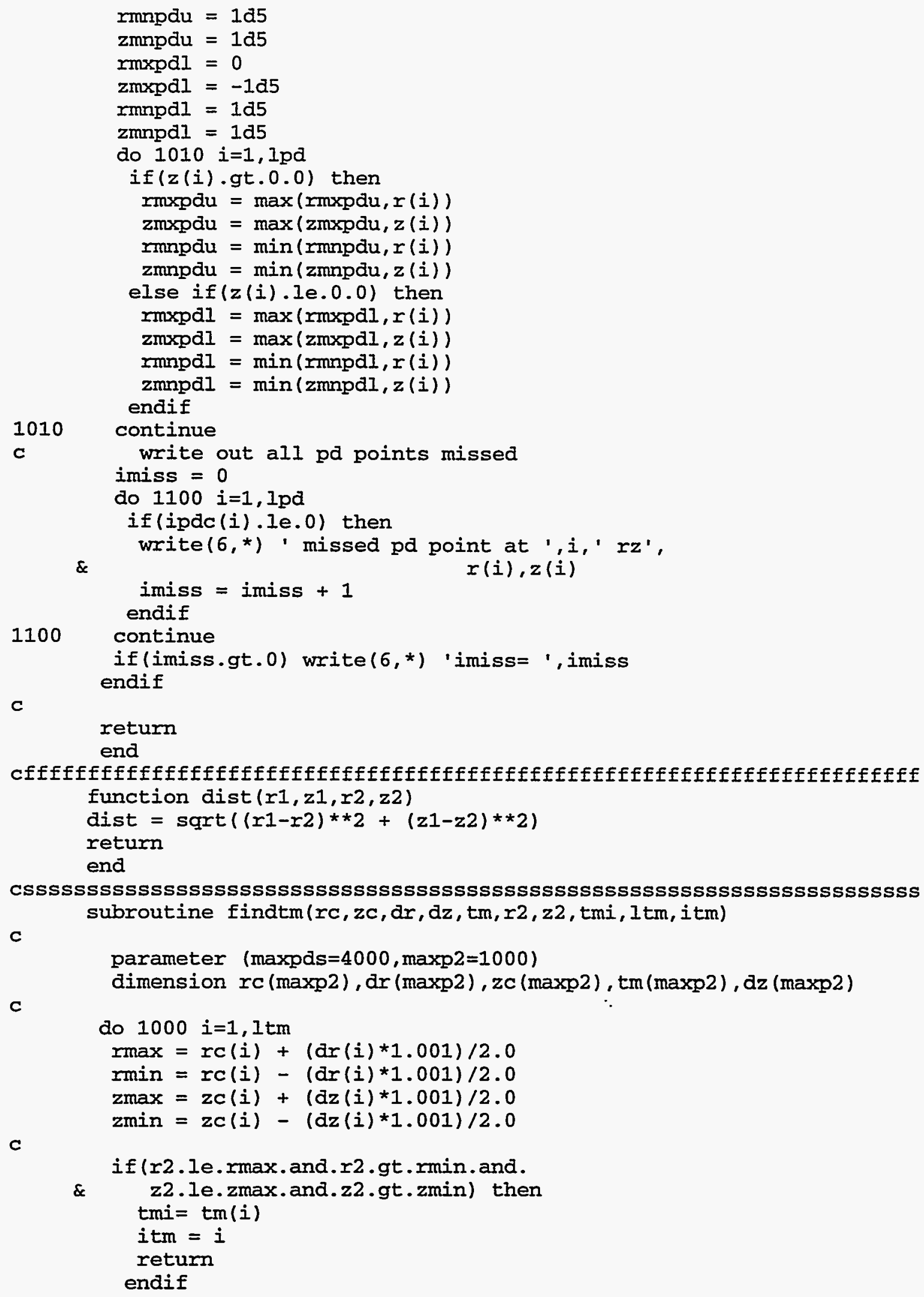

c

return end

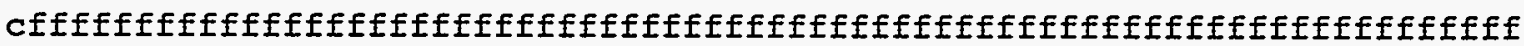

function dist $(r 1, z 1, r 2, z 2)$

dist $=\operatorname{sqrt}((r 1-r 2) * * 2+(z 1-z 2) * * 2)$

return end

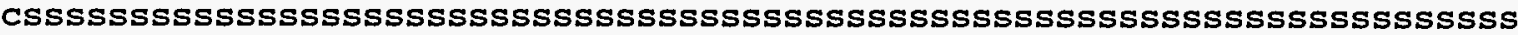
subroutine findtm(rc, zc, dr, dz,tm, r2, z2, tmi, ltm, itm)

C

parameter (maxpds $=4000, \operatorname{maxp} 2=1000$ )

dimension $r c(\operatorname{maxp} 2), \mathrm{dr}(\operatorname{maxp} 2), z c(\operatorname{maxp} 2), \operatorname{tm}(\operatorname{maxp} 2), \mathrm{dz}(\operatorname{maxp} 2)$

$\mathbf{C}$

do $1000 i=1,1 \mathrm{tm}$

$\max =r c(i)+(\operatorname{ar}(i) * 1.001) / 2.0$

$\operatorname{rmin}=r c(i)-(\operatorname{dr}(i) * 1.001) / 2.0$

$z \max =z c(i)+(d z(i) * 1.001) / 2.0$

$\operatorname{zmin}=z c(i)-(d z(i) * 1.001) / 2.0$

c

if (r2. le.rmax. and.r2.gt.rmin. and.

$\&$ z2.le.zmax.and.z2.gt.zmin) then $\operatorname{tmi}=\operatorname{tm}(i)$

itm $=i$

return

endif 
Table E.1. Listing of MK-FD.F computer code (continued)

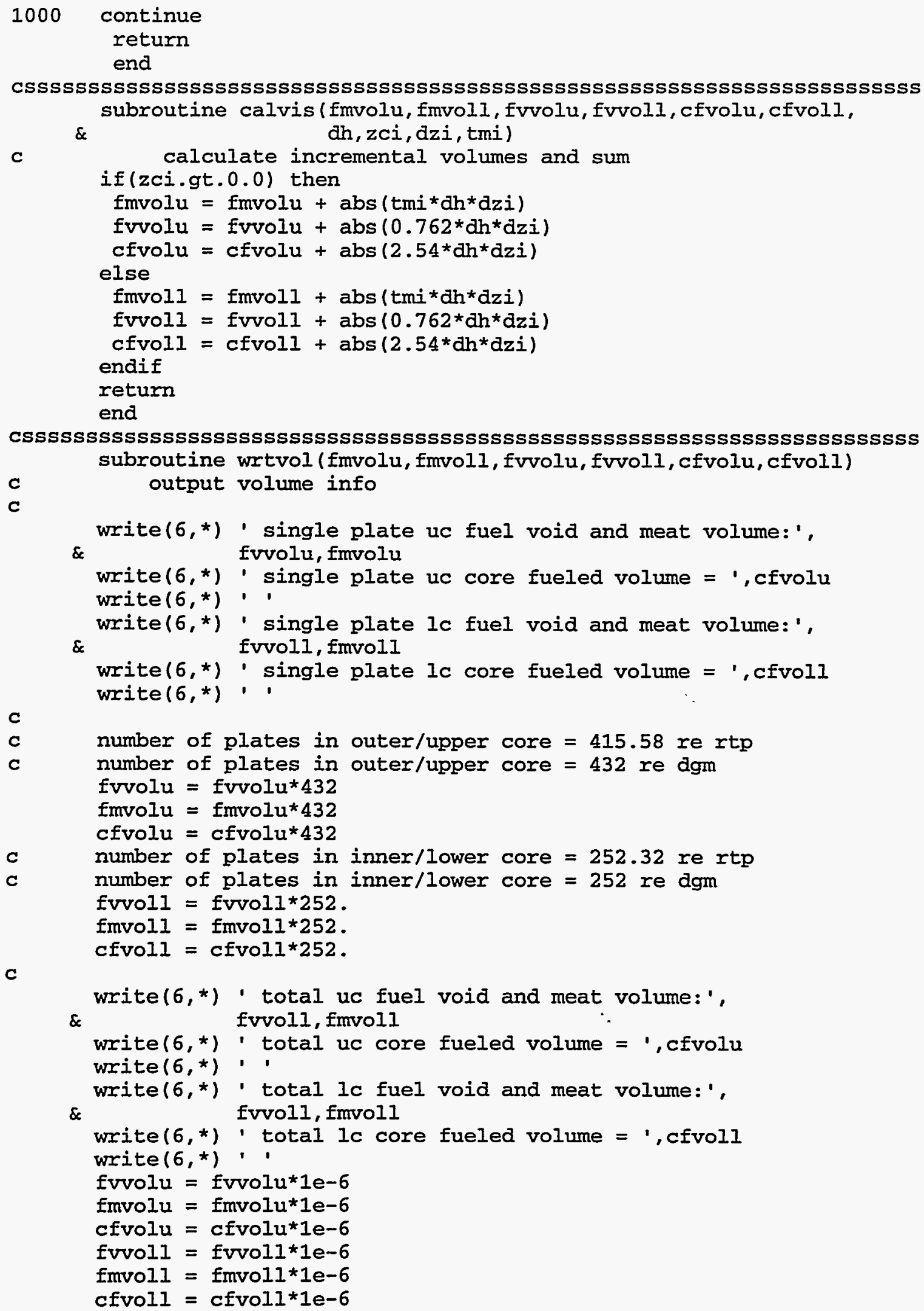

C

C

C

number of plates in outer/upper core $=415.58$ re rtp number of plates in outer/upper core $=432 \mathrm{re} \mathrm{dgm}$ fvvolu $=$ fvrolu* 432

fmvolu $=$ fmvolu $* 432$

C

$\&$

write $(6, *)$ ' total uc fuel void and meat volume:',

write $\left(6,{ }^{*}\right)$ ' total uc core fueled volume $=$, cfvolu write $(6, *)$, '

$\&$

write $(6, *)$ ' total $1 \mathrm{c}$ fuel void and meat volume:', fvvol1, fmvoll

write $(6, *)$ ' total lc core fueled volume = , cfvoll write $(6, *)$, '

fvvolu $=$ fvrolu*1e-6

fmvolu $=$ fmvolu*1e-6

cfvolu $=$ cfvolu*1e-6

fvroll $=$ fvvoll*1e-6

fmvoll $=$ fmvoll $1 * 1 e-6$

cfvoll = cfvol $1 * 1 e-6$ 
Table E.1. Listing of MK-FD.F computer code (continued)

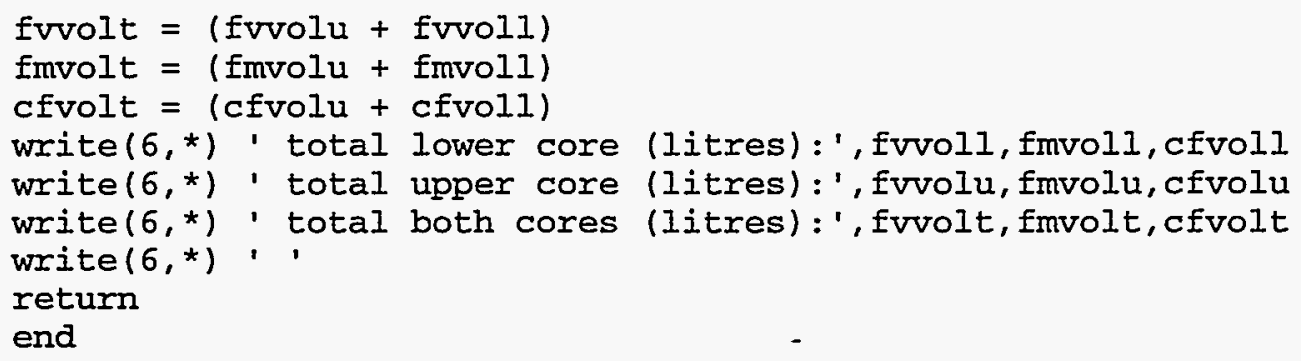




\section{E.15}

\section{Table E.1. Listing of MK-FD.F computer code (continued)}

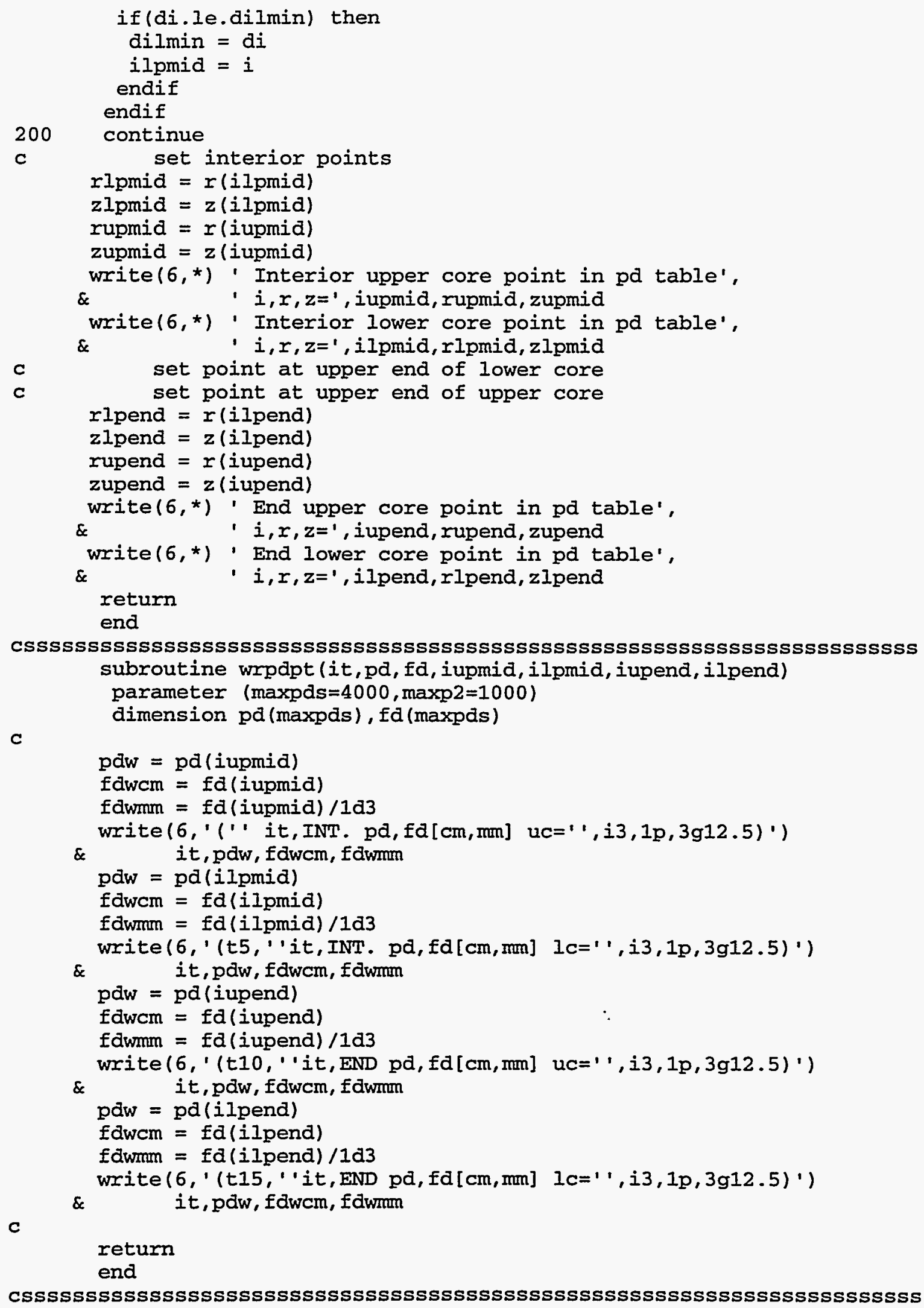




\section{E.16}

\section{Table E.1. Listing of MK-FD.F computer code (continued)}

subroutine wrtmax (it,pd, fd, lpd,pdm, fdm)

parameter (maxpds $=4000, \operatorname{maxp} 2=1000$ )

dimension pd (maxpds), fd (maxpds), pdm (8), fdm(8)

C

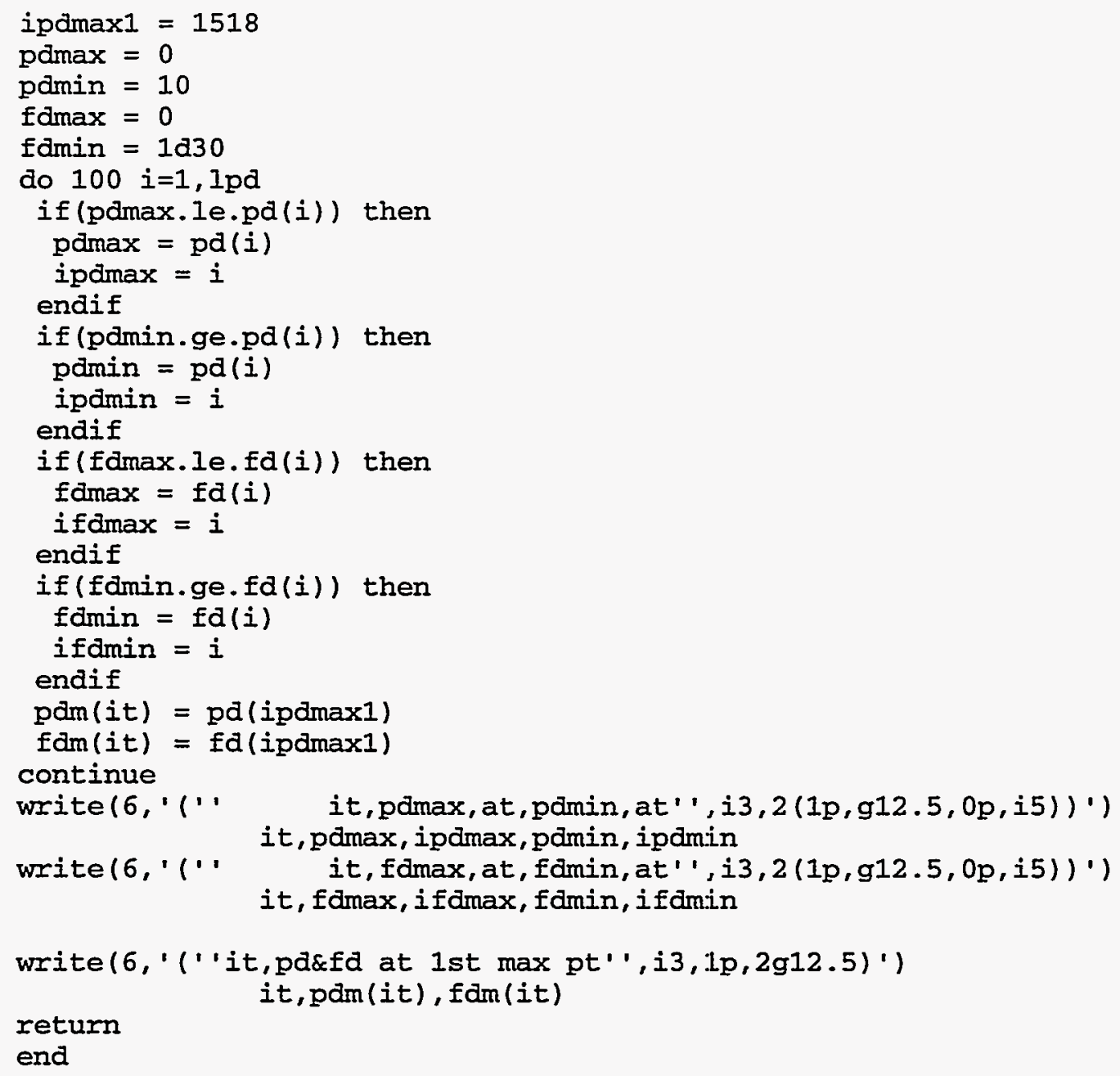


Table E.2. Format of output file for use by LATa

Line 1 'Time=', TIME

Line 2-end Three entries in free form format

Entry Variable Units

1 RADIUS (mm)

2 Z (mm)

3 FISSION DENSITY $\left(\mathrm{f} / \mathrm{mm}^{3}\right)$ data set.

aThis data set will have the same order as the relative power density

Table E.3. Format for input file for surface density distributiona

\begin{tabular}{ccc}
\hline Entry & Variable & Units \\
& & \\
1 & SURFACE DENSITY & $\left(\mathrm{mg} / \mathrm{mm}^{2}\right)$ \\
2 & RADIUS & $(\mathrm{mm})$ \\
3 & SPAN & $(\mathrm{mm})$ \\
4 & $\mathrm{Z}$ & $(\mathrm{mm})$ \\
\hline \multicolumn{3}{l}{ aThis data set must have the same order as the meat thickness data set. }
\end{tabular}

Table E.4 Format of input file for burnup fraction distribution ${ }^{a}$

\begin{tabular}{ccc}
\hline Entry & Variable & Units \\
& & \\
1 & RADIUS & $(\mathrm{mm})$ \\
2 & SPAN & $(\mathrm{mm})$ \\
3 & Z & $(\mathrm{mm})$ \\
4 & BURNUP & (unitless) \\
\hline \multicolumn{3}{l}{ aThis data set must have the same order as the meat thickness data set. }
\end{tabular}


E.18

Table E.5. Format of output file for use with TECPLOT

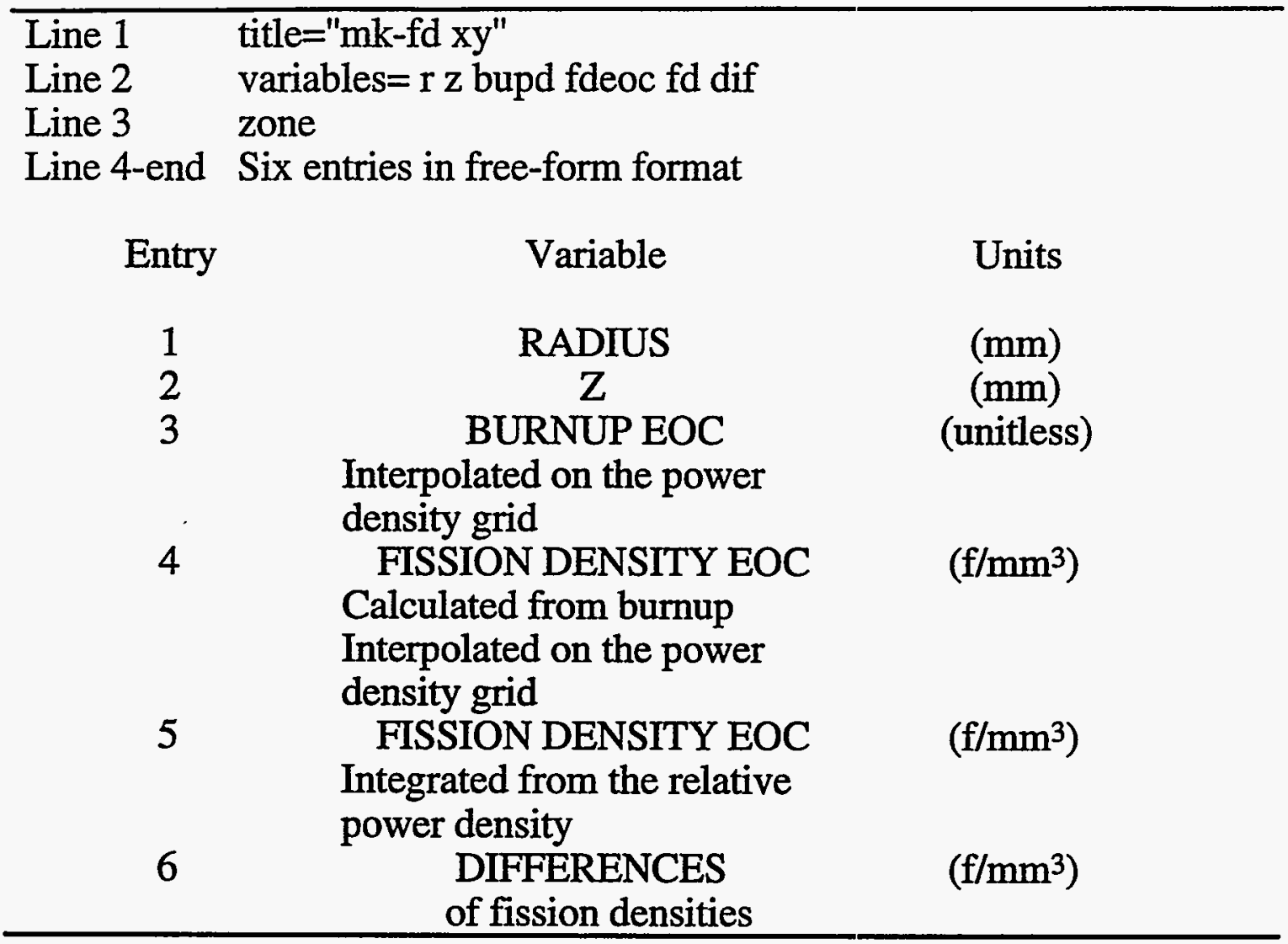




\section{INTERNAL DISTRIBUTION}

1-5. J. H. Campbell

6. K. W. Childs

7. G. L. Copeland

8. J. D. Freels

9-13. G. E. Giles

14. D. M. Hetrick

15-18. R. L. Johnson

19. J. R. Kirkpatrick

20. M. A. Kuliasha

21. D. G. Morris

22. D. L. Selby
23-25. C. H. Shappert

26. C. D. West

27. P. T. Williams

28. G. L. Yoder

29. Central Research Library Document Reference Section

30. ORNL $/ Y-12$ Technical Library 31-32. Laboratory Records Department

33. Laboratory Records, ORNL-RC

34. ORNL Patent Office

\section{EXTERNAL DISTRIBUTION}

35. U.S. Department of Energy, Oak Ridge Operations Office, FEDC, MS-8218, P.O. Box 2009, Oak Ridge, TN, 37831-8218

36. Office of Scientific and Technical Information, P.O. Box 62, Oak Ridge, TN 37831 
